\title{
Design and development of group 13 precursors for improved vapour deposition of metal nitride thin films
}

by

\author{
Sydney Cristina Buttera
}

A thesis submitted to the Faculty of Graduate and Postdoctoral Affairs in partial fulfillment of the requirements for the degree of

Doctor of Philosophy

in

Chemistry

CARLETON UNIVERSITY

Ottawa, Ontario

(C) 2020

Sydney Cristina Buttera 
This thesis is dedicated to my parents, Claudia and Rob Buttera. 


\section{Abstract}

Vapour phase deposition techniques such as atomic layer deposition (ALD) and chemical vapour deposition (CVD) are important for the deposition of thin films for a wide range of applications. Metal oxides and metal nitrides, particularly, can be used as passivation layers and dielectrics, and due to their use in increasingly small microelectronic devices, their deposition must be precise, conformal, and of high purity. It is non-trivial to deposit pure materials without impurities by ALD; several factors including temperature, exposures, and precursor(s) must be considered. This work examines how precursor design can reduce impurities in deposited films. An initial thermal study of an indium tris(guanidinate) precursor by high-resolution NMR (HR-NMR) and thermogravimetric analysis (TGA) demonstrated its thermal instability as a function of long-term heating, following which simpler group 13 precursors were synthesized and studied. Both $\mathrm{Al}\left(\mathrm{NMe}_{2}\right)_{3}$ and $\mathrm{Ga}\left(\mathrm{NMe}_{2}\right)_{3}$ were designed due to their lack of metal-carbon bonds and were used to deposit metal oxide and metal nitride films, respectively, with very low impurities by ALD. Methylamines were studied computationally in comparison to ammonia as a possible replacement for a nitrogen co-reagent, though CVD experiments confirmed their favoured reactivity in the gas phase as opposed to at the surface. Other bidentate group 13 ligands were also studied where a new In(III) triazenide produced pure, epitaxial indium nitride by ALD, and MeNacNac ligands were used to develop novel $\mathrm{Al}$ precursors including $\mathrm{Al}\left({ }^{\mathrm{MeNacNac}}\right)\left(\mathrm{NMe}_{2}\right)_{2}$ and $\mathrm{Al}\left({ }^{\mathrm{Me} N a c N a c}\right)(\mathrm{r}, \mathrm{MeNacNac})$. Ultimately, a simple $\mathrm{AlH}_{2}\left(\mathrm{NMe}_{2}\right)$ precursor was designed due to its metal-nitrogen bonds and the addition of the hydride ligand to maintain a reducing atmosphere during depositions and resist AlN film oxidation. Pure aluminum nitride films were deposited by ALD using this precursor and ammonia plasma as a co-reagent. Generally, this work emphasizes the importance of smart precursor design to produce pure, high-quality group 13 nitride thin films. It demonstrates the value in developing new ALD precursors, but doing so in a methodical and logical way. This methodology could be extended to other metal centres to develop a variety of precursors and ALD process that will deposit pure, impurity-free, and high-quality films in a cost- and time-efficient manner. 


\section{Preface}

This preface provides full bibliographical details for each article included in this thesis, as well as whether the article is reproduced in whole or in part. Use of copyrighted material is likewise acknowledged here. When citing material from this thesis, please cite the article relevant to the chapter, if the chapter is based on a publication.

Pursuant to the Integrated Thesis policy of Carleton University, the supervisor (Seán T. Barry) and the "student" (Sydney C. Buttera) confirm that the student was fully involved in setting up and conducting the research, obtaining data and analyzing results, as well as preparing and writing the material presented in the co-authored articles(s) integrated in the thesis. Additionally, the supervisor confirms the information provided by the student in this preface.

\section{Chapter 2}

Buttera, S. C.; Rönnby, K.; Pedersen, H.; Ojamäe, L.; Barry, S. T. Thermal Study of an Indium Trisguanidinate as a Possible Indium Nitride Precursor. J. Vac. Sci. Technol. A Vacuum, Surfaces, Film. 2018, $36 \quad$ (1), $01 \mathrm{~A} 101$. https://doi.org/10.1116/1.5002634

This article is wholly reproduced and edited for formatting and clarity of presentation. Sydney C. Buttera performed all experimentation for this publication outside of the modelling, which was performed by Karl Rönnby and Lars Ojamäe. The publication was written by Seán T. Barry.

\section{Chapter 3}

Part I - Buttera, S. C.; Mandia, D. J.; Barry, S. T. Tris(Dimethylamido)Aluminum(III): An Overlooked Atomic Layer Deposition Precursor. J. Vac. Sci. Technol. A Vacuum, Surfaces, Film. 2017, 35 (1), 01B128. https://doi.org/10.1116/1.4972469. 
This article is wholly reproduced and edited for formatting and clarity of presentation. Sydney C. Buttera performed all experimentation for this publication including precursor design and synthesis, thermogravimetric analysis, and thin film deposition. David J. Mandia performed X-ray photoelectron spectroscopy measurements and their analyses, as well as assisted with manuscript preparation. This publication was written by Sydney C. Buttera.

Part II - Rouf, P.; O’Brien, N. J.; Buttera, S. C.; Martinovic, I.; Bakhit, B.; Martinsson, E.; Palisaitis, J.; Hsu, C.-W.; Pedersen, H. Epitaxial GaN Using $\mathrm{Ga}\left(\mathrm{NMe}_{2}\right)_{3}$ and $\mathrm{NH}_{3}$ Plasma by Atomic Layer Deposition. J. Mater. Chem. C 2020, 3, 1101-1134. https://doi.org/10.1039/D0TC02085K.

This article is wholly reproduced and edited for formatting and clarity of presentation. Sydney C. Buttera performed and analyzed all thermal characterization of the precursor, including calculation of the 1 Torr temperature, by thermogravimetric analysis and differential scanning calorimetry. Polla Rouf performed the majority of the precursor synthesis and perform SEM, XPS, and XRD measurements. Nathan J. O'Brien aided with synthesis and Erik Martinsson performed absorption measurements. Babak Bakhit performed ERBA/RBS while Ivan Martinovic, Justinas Palisaitis, and Chih-Wei Hsu collected TEMs. This publication was written by Polla Rouf.

\section{Chapter 4}

Rönnby, K.; Buttera, S. C.; Rouf, P.; Barry, S. T.; Ojamäe, L.; Pedersen, H. Methylamines as Nitrogen Precursors in Chemical Vapor Deposition of Gallium Nitride. J. Phys. Chem. C 2019, 123 (11), 6701-6710. https://doi.org/10.1021/acs.jpcc.9b00482.

This article is wholly reproduced and edited for formatting and clarity of presentation. Sydney C. Buttera designed and performed all chemical vapour deposition experiments and collected all optical microscopy images. Computational experiments were performed by Karl Rönnby and Lars Ojamäe. Polla Rouf performed grazing-incidence X-ray diffraction experiments on thin films. This publication was largely written by Karl Rönnby while all other collaborators contributed for sections of the publication that pertained to their experiments. 


\section{Chapter 5}

Part I - O'Brien, N. J.; Rouf, P.; Samii, R.; Ronnby, K.; Buttera, S. C.; Hsu, C.W.; Ivanov, I. G.; Kessler, V.; Ojamäe, L.; Pedersen, H. In-Situ Activation of an Indium(III) Triazenide Precursor for Epitaxial Growth of Indium Nitride by Atomic Layer Deposition. Chem. Mater. 2020. https://doi.org/10.1021/acs.chemmater.9b05171.

This article is wholly reproduced and edited for formatting and clarity of presentation. Sydney C. Buttera performed all thermolysis experiments, including thermogravimetric analysis and differential scanning calorimetry, as well as their analyses and calculation of the 1 Torr temperature of all precursors. Nathan J. O'Brien developed and Rouzbeh Samii developed and characterized the precursor while Polla Rouf performed deposition experiments and their associated characterizations (XPS, SEM, XRR). Karl Rönnby and Lars Ojamäe performed DFT calculations related to the thermal decomposition of precursors. Chih-Wei Hsu characterized the films electronically, Ivan Ivanov performed Raman spectroscopy, and Vadim Kessler performed XRD. This publication was written by Nathan J. O'Brien with the aid of other contributors related to their experiments and measurements.

Part II - Buttera, S. C.; Masuda, J. D.; Barry, S. T. Novel Aluminum MeNacNac Compounds as Possible Precursors for Atomic Layer Deposition. Manuscript in progress.

This article is a manuscript in progress. Sydney C. Buttera performed all syntheses, thermal characterizations by thermogravimetric analysis, and NMR boilup experiments. Jason C. Masuda collected solid state molecular structures by single crystal X-ray diffraction. This manuscript was written by Sydney C. Buttera.

\section{Chapter 6}

Buttera, S. C.; Rouf, P.; Deminskyi, P.; Pedersen, H.; Barry, S. T. A Low Cost, High Efficiency TMA-replacement for the Deposition of Pure Aluminum Nitride by Atomic Layer Deposition. Manuscript in progress.

This article is a manuscript in progress. Sydney C. Buttera designed the precursor, performed and analyzed thermogravimetric analysis including the collection of the 1 Torr temperature, and designed and performed all ALD experiments. Polla Rouf 
and Petro Deminskyi collected and analyzed all GIXRD data. Polla Rouf calculated AlN stress and strain and collected/analyzed XPS data. SEM data was collected by Petro Deminskyi. This manuscript was written by Sydney C. Buttera. 


\section{Acknowledgements}

Writing this thesis and looking back on all this work is the greatest representation of the fact that I could have never done this on my own. I am eternally grateful to every single person who contributed to this work, taught me along the way, or was there as sometimes much-needed and always much-appreciated support.

Firstly, I have to acknowledge my supervisor Dr. Seán T. Barry. Seán, without your trust in me as a post-first year student and incalculable support over the following eight years through to my $\mathrm{PhD}$ studies, this thesis would not exist. Your encouragement of my research ideas and belief in my abilities did not go unnoticed and will be forever appreciated.

I hesitate to use the term co-workers, because the people I worked with every day in the Barry lab are far more accurately described as friends. I cannot count the amount of times I've cried laughing in the lab or the office, or the number of times I've gotten help with a research problem from one of you spectacular people. Work didn't feel like work when I got to hang out with all of you crazy guys every day. Jason Coyle, David Mandia, Michael Land, David Zanders, Aya Kadri, Joey Zurakowski, Atilla Varga, Eden Goodwin, and all other previous Barry lab group members, working with you all has been a distinct pleasure and I wish you all nothing but the best, both in research and in life. To the people who have been there since the beginning, I owe an extra level of gratitude that I must highlight. Peter Gordon, you've provided endless laughs and I cannot thank you enough for the years of support you've given me. Matt Griffiths and Goran Bačić, you are some of the best chemists I know, but more importantly, you are truly cherished friends and I love you both. Finally, to Peter Pallister I must also express my sincerest gratitude. Peter, you were my mentor when I first started in Seán's lab and you taught me what it is to be a great scientist, with, I must note, a colossal measure of patience. I have undoubtably found a lifelong friend in you.

In the Carleton chemistry department, I also want to acknowledge Daniel Sun, Maria DeRosa, and Bob Burk. Daniel, your teaching labs are a blast and 
working with you was always a great pleasure. Being a TA for your courses was some of the most fun I've had at Carleton and pushed me in a teaching direction for which I've found great love and passion. Maria, you have always been supportive of my teaching endeavours and have backed my ideas to get them off the ground; I cannot express to you how grateful I am for this. Bob, you're the reason I switched into chemistry in the first place. Your excellence and support in all things teaching does not go unnoticed or unappreciated. I would also thank the rest of the chemistry department and Peter, Susa, Tanya, Chantelle, and Caitlyn for always keeping research and studies running smoothly.

My opportunity to travel and work in Sweden with Henrik Pedersen's group at Linköping University was a highlight of my $\mathrm{PhD}$ studies and I am so grateful to have had this experience. Henrik, thank you for welcoming me into your group and to all your group members for their immeasurable contributions to research projects. Polla Rouf, Petro Deminskyi, Karl Rönnby, Nathan O'Brien, and Hama Nadhom, you are all great friends and I value all the time we've had to work together in Sweden and across the ocean. I found another chemistry family and a second home in Linköping and will always have the fondest memories of this time.

My family are the absolute greatest people that I know, and to say that I could not have done this without them is a gross understatement. My parents are two of the smartest people I've ever met, but always reminded a very stressed-outto-be-perfect child and teenager and adult that we can only ever do our best. For as long as I can remember, my dad has been telling me that no matter what, "Syd, the sun is still going to come up tomorrow" and he's right. It always does, and learning that, paired with my parents' unwavering support, has gotten me all the way through school starting in kindergarten at age 4 to a $\mathrm{PhD}$ at 27. Mom, I can't say thank you enough for all the help you've given me with teaching particularly, but more importantly, for being a constant support system throughout my life. The hugs and talks and cups of tea mean more than I can say. Dad, like I've said before, thank you for always keeping me laughing and teaching me not to take life too seriously. You remind me to always look at the big picture and to not sweat the little things, which I've been working on for the past 27 years and could not achieve without you. Christa, you are my sister, but you're also my best friend. I can't tell you how much I value all of our hangout time, from watching hours of The OC and Harry Potter, to having rice paper roll and Twilight Saturday nights. Sometimes it was to distract us from school stress, but I cherish the time most for the reason that you're a beautiful person and any time with you is well spent. I love you all so so much. 
At the risk of writing the world's longest thesis acknowledgements, I'll try to keep this last one short. Brad, before you were my husband, you were my study and homework partner; now you're my everything partner. You have given me so much support and motivation over the past eight years, and I am appreciative of that beyond measure. Thank you for being an extraordinary person; I could not have done this without you. 


\section{Contents}

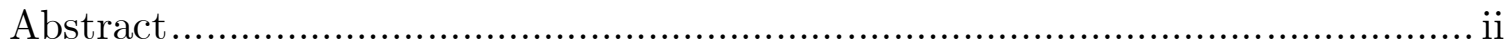

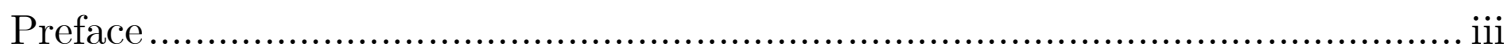

Acknowledgements..................................................................... vii

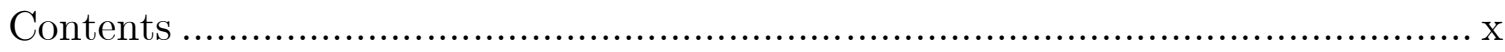

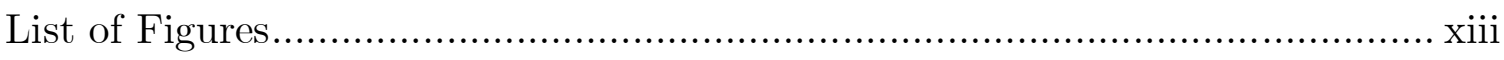

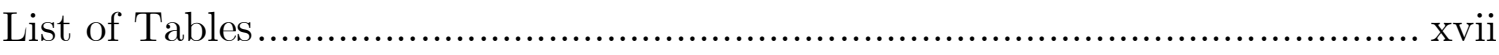

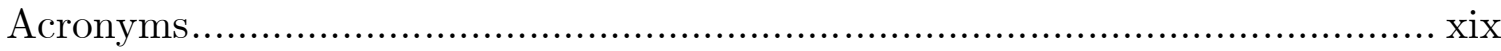

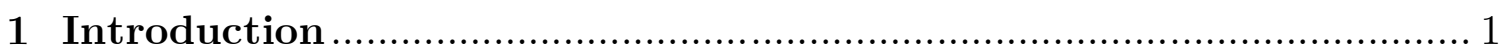

1.1 Group 13 Nitrides ....................................................... 1

1.2 Vapour Phase Thin Film Deposition.......................................... 3

1.2.1 Chemical Vapour Deposition ............................................ 4

1.2.2 Atomic Layer Deposition ................................................ 6

1.3 Thermal Characterization of Precursors ........................................11

1.3.1 Thermogravimetric Analysis............................................11

1.3.2 Differential Scanning Calorimetry ......................................... 14

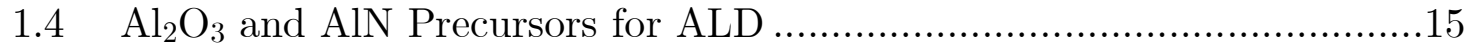

1.4.1 Aluminum Trichloride ............................................... 16

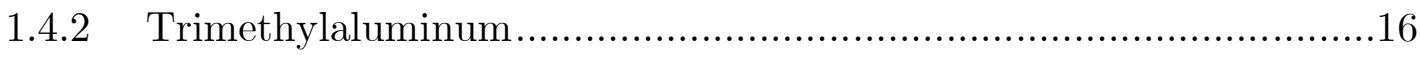

1.4.3 Amidinates and Guanidinates..........................................20

1.4.4 Other Aluminum Precursors...............................................23

2 Thermal Study of an Indium Trisguanidinate as a Possible Indium Nitride Precursor ......................................................... 28

2.1 Abstract ........................................................................29 


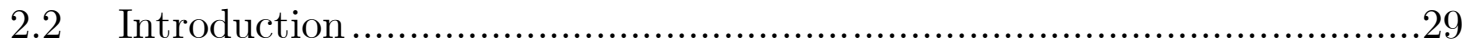

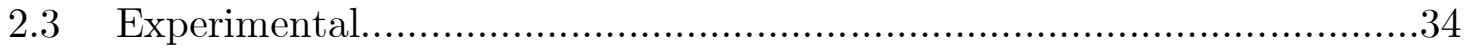

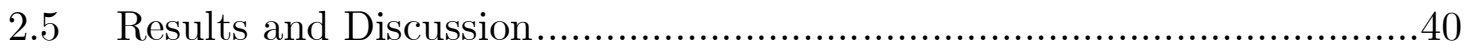

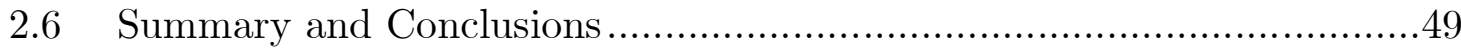

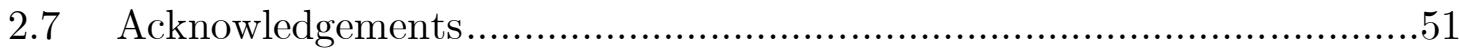

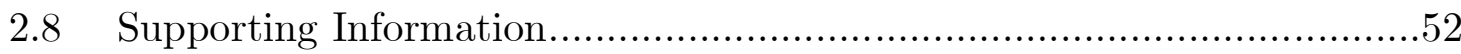

3 Part I - Tris(dimethylamido)aluminum(III): An Overlooked ALD

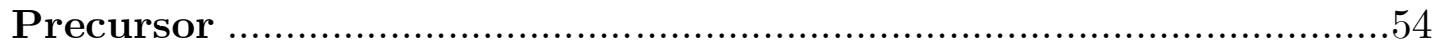

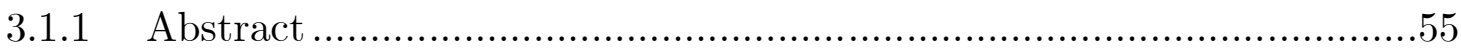

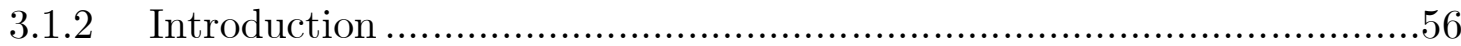

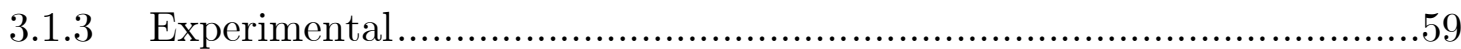

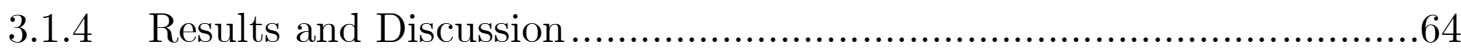

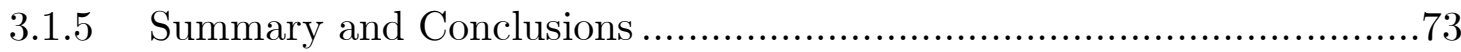

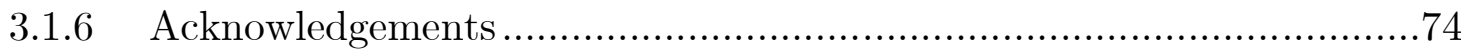

3 Part II - Epitaxial GaN using $\mathrm{Ga}\left(\mathrm{NMe}_{2}\right)_{3}$ and $\mathrm{NH}_{3}$ plasma by

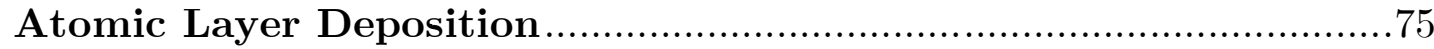

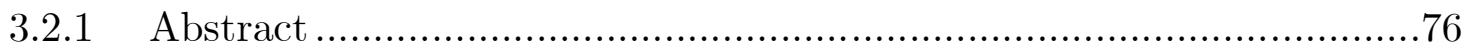

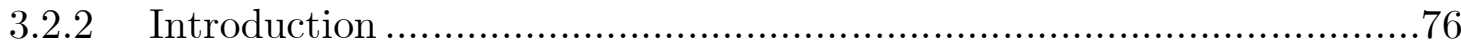

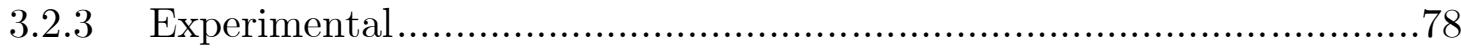

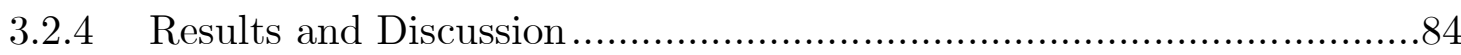

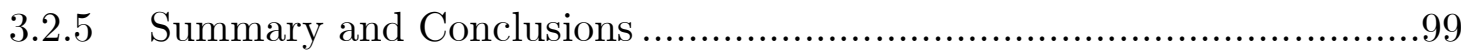

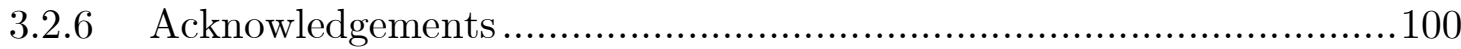

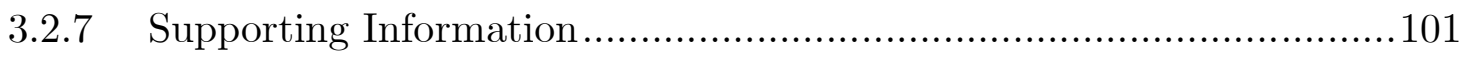

4 Methylamines as Nitrogen Precursors in Chemical Vapour

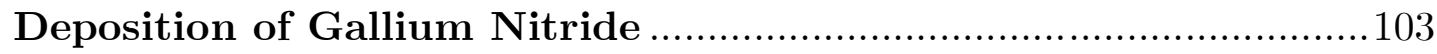

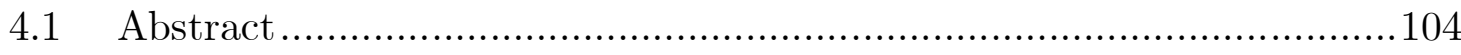

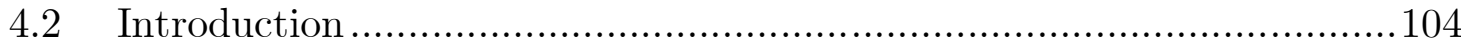

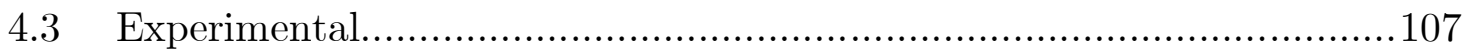

4.4 Results and Discussion...............................................................111

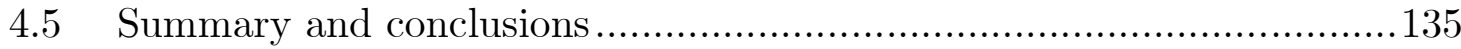

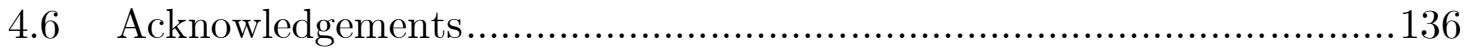

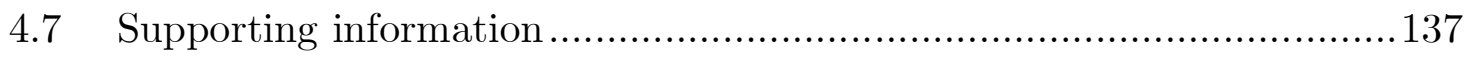


5 Part I - In-situ Activation of an Indium(III) Triazenide Precursor for Epitaxial Growth of Indium Nitride by Atomic Layer

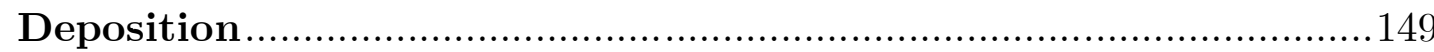

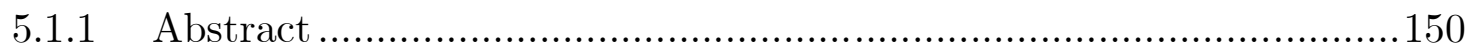

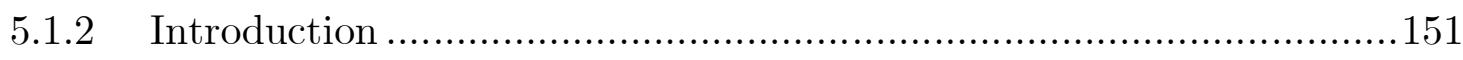

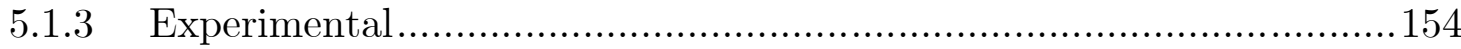

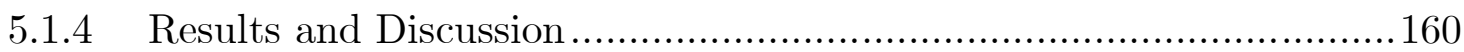

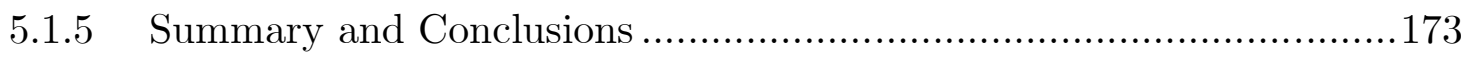

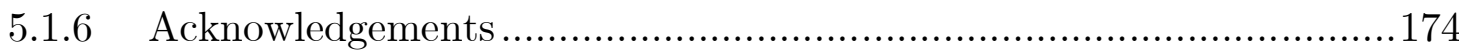

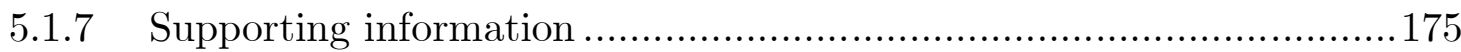

5 Part II - Novel Aluminum MeNacNac Compounds as Possible Precursors for Atomic Layer Deposition ...........................................193

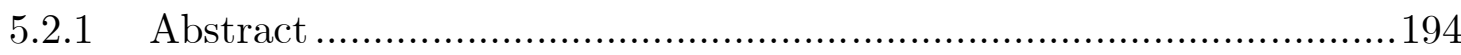

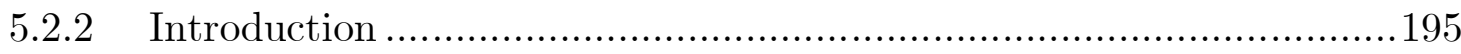

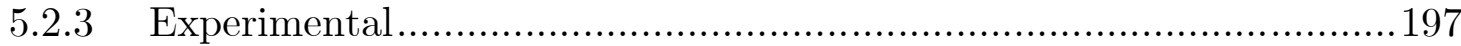

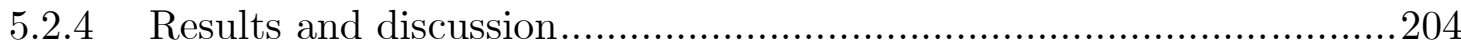

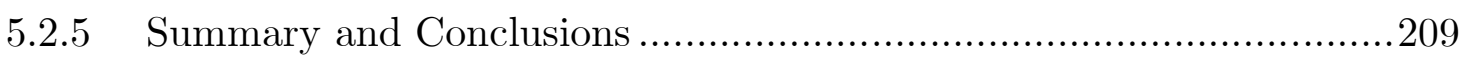

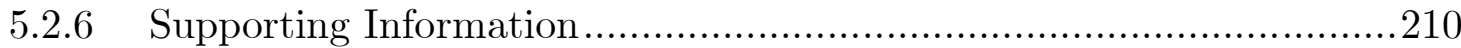

6 A Low Cost, High Efficiency TMA-replacement for the Deposition of Pure Aluminum Nitride by Atomic Layer Deposition...............224

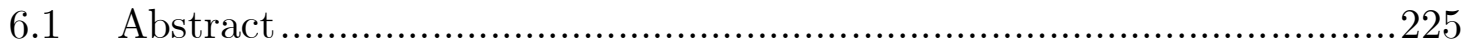

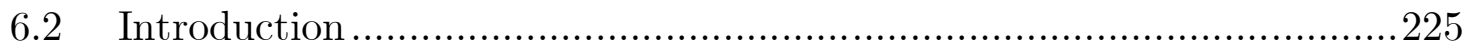

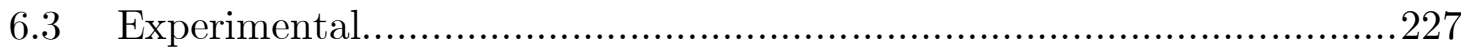

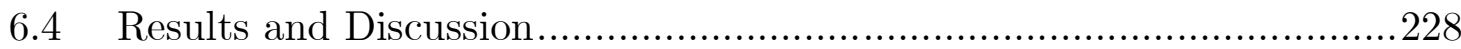

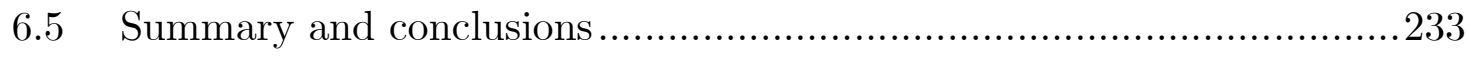

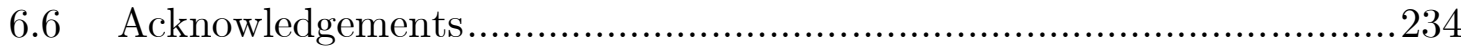

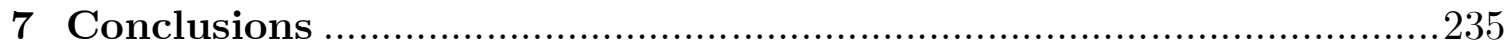

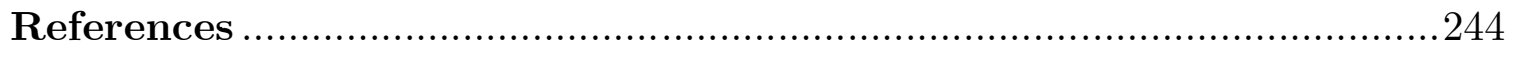




\section{List of Figures}

1.1 Generalized growth behaviour of ALD vs. CVD processes .............. 9

1.2 TGA traces demonstrating volatilization and decomposition ......... 12

$1.3 \quad$ DSC trace of an ALD precursor ..................................... 15

1.4 Chemical reactions for ALD of aluminum oxide using TMA and

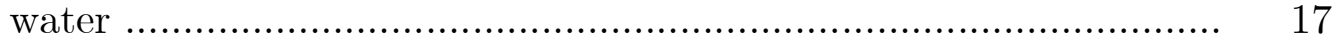

1.5 Graphic for $\mathrm{Al}_{2} \mathrm{O}_{3}$ ALD using TMA and water .......................... 19

1.6 Resonance structures of amidinate and guanidinate ligands ........... 21

1.7 Insertion of a carbodiimide over a metal alkyl or amide ................ 21

1.8 Synthesis of aluminum guanidinate precursors ............................ 22

1.9 Array of ALD precursors relevant to this thesis ....................... 26

2.1 Elimination of diisopropylcarbodiimide from trisguanidinato group

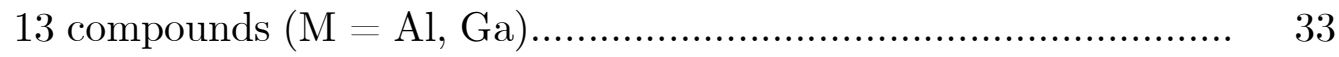

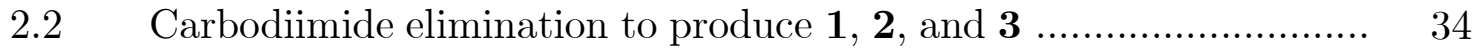

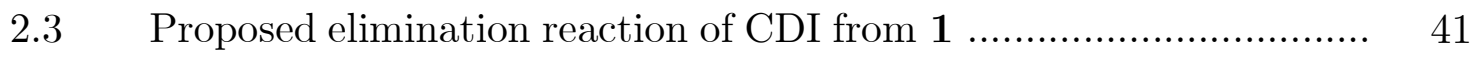

2.4 Temperature dependence of CDI elimination from $\mathbf{1}$ to $\mathbf{2}$ and from

2 to 3 .......................................................................... 44

2.5 Partial pressures from CDI elimination of $\mathbf{1}$ to produce $\mathbf{2}$ and $\mathbf{3} \ldots . .45$

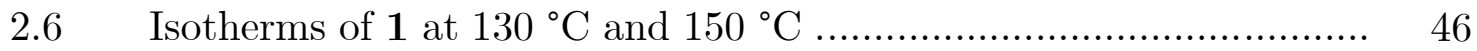

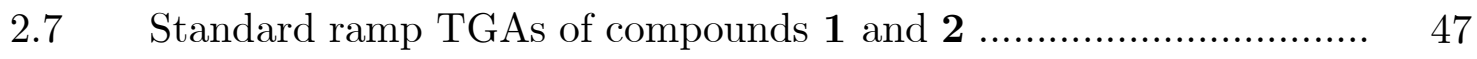

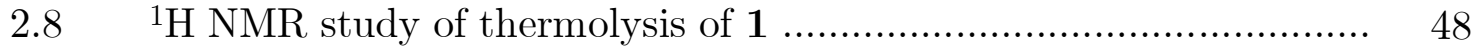

2.9 Converged structures of compounds 1-3 and intermediates ........... 52

3.1 Common decomposition pathways for amidinate and guanidinate compounds........................................................... 58

3.2 Thermogravimetric data for TDMAA …................................ 65

3.3 Saturation curves for TDMAA producing aluminum oxide ............ 66

3.4 XPS of aluminum oxide films deposited using TDMAA ................ 67

3.5 Saturation curves for TDMAA with $\mathrm{N}_{2}$ plasma .......................... 68

3.6 XPS of films deposited using TDMAA and $\mathrm{N}_{2}$ plasma .................. 70

3.7 HR-XPS of films deposited using TDMAA and $\mathrm{N}_{2}$ plasma ............ 72

$3.8 \quad$ Synthesis of 4 ........................................................... 80

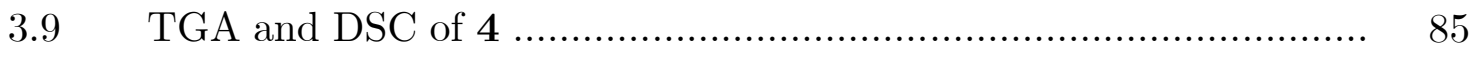


3.10 Saturation of curves of 4 and $\mathrm{NH}_{3}$ to deposit GaN

3.11 XRD, GIXRD, $\varepsilon-\sin ^{2} \psi$ plot, and SEM of GaN deposited using 4 and $\mathrm{NH}_{3}$ plasma

3.12 HR-XPS of GaN deposited using 4 and $\mathrm{NH}_{3}$ plasma

3.13 Absorption spectrum, valence band spectrum, Fermi level region, and schematic band diagram for GaN deposited using 4 and $\mathrm{NH}_{3}$ plasma

3.14 XRD, GIXRD, and pole figures of GaN deposited using 4 and $\mathrm{NH}_{3}$ plasma

3.15 STEM-HAADF from GaN films deposited using 4 and $\mathrm{NH}_{3}$ plasma

3.16 Plot of $\ln$ (vapour pressure) vs. $1 / \mathrm{T}$ for 4

3.17 XRC of GaN deposited using 4 and $\mathrm{NH}_{3}$ plasma

3.18 Top-view SEM of GaN ( $4+\mathrm{NH}_{3}$ plasma) on $\mathrm{SiC}$

3.19 XPS of GaN deposited using $\mathbf{4}$ and $\mathrm{NH}_{3}$ plasma deposited at 200 ${ }^{\circ} \mathrm{C}$ after sputtering

4.1 Optimized geometries and bond lengths for $\mathrm{NH}_{\mathrm{x}}\left(\mathrm{CH}_{3}\right)_{\mathrm{n}-\mathrm{x}}(\mathrm{n}=3,2,1)$

4.2 Optimized geometries for adsorptions of undecomposed precursors at the GaN (0001) surface

4.3 GIXRD and opitcal micrscopy of GaN deposited by CVD at $850{ }^{\circ} \mathrm{C}$ using $\mathrm{Ga}\left(\mathrm{CH}_{3}\right)_{3}$ and $\mathrm{NH}_{3}$ with $\mathrm{N} / \mathrm{Ga}=570$

4.4 SEM of GaN deposited by CVD at $850{ }^{\circ} \mathrm{C}$ using $\mathrm{Ga}\left(\mathrm{CH}_{3}\right)_{3}$ and $\mathrm{NH}_{3}$ with $\mathrm{N} / \mathrm{Ga}=570$

4.5 SEM from CVD samples grown at $925{ }^{\circ} \mathrm{C}$ from $\mathrm{Ga}\left(\mathrm{CH}_{3}\right)_{3}$ and $\mathrm{N}\left(\mathrm{CH}_{3}\right)_{3}$ using $\mathrm{N} / \mathrm{Ga}=275, \mathrm{~N} / \mathrm{Ga}=570$, and $\mathrm{N} / \mathrm{Ga}=1100$

4.6 SEM from CVD samples grown at $925{ }^{\circ} \mathrm{C}$ from $\mathrm{Ga}\left(\mathrm{CH}_{3}\right)_{3}$ and $\mathrm{N}\left(\mathrm{CH}_{3}\right)_{3}$ using $\mathrm{N} / \mathrm{Ga}=570$ with low and high precusor flow

4.7 Schematics of a possible growth mechanism for the observed cones grown by CVD using $\mathrm{Ga}\left(\mathrm{CH}_{3}\right)_{3}$ and $\mathrm{N}\left(\mathrm{CH}_{3}\right)_{3}$

4.8 Hydrogen terminated gallium nitride clusters

4.9 Enthalpy and free energy of transition state and reaction for BC2 .

4.10 Reaction enthalpy and free energy for gas phase and adsorption reactions from unimolecular reactions and decomposition of methylamines, and adduct formation between methyalmines with TMG with subsequent alkane elimination

4.11 Transition state enthalpy and free energy for all gas phase and adsorption reactions from unimolecular reactions and decomposition of methylamines, and adduct formation between methyalmines with TMG with subsequent alkane elimination .........

4.12 Reactant and product geometries for the rearrangement of $\mathrm{NCH}_{3}$ to $\mathrm{NHCH}_{2}$ 
4.13 Geometry optimized structures for Lewis adducts formed between $\mathrm{NH}_{3-\mathrm{x}}\left(\mathrm{CH}_{3}\right)_{\mathrm{x}}$ and $\mathrm{Ga}\left(\mathrm{CH}_{3}\right)_{3}$

4.14 Geometry optimized structures for Lewis adducts formed between $\mathrm{NH}_{3-\mathrm{x}}\left(\mathrm{CH}_{3}\right)_{\mathrm{x}}$ and $\mathrm{Al}\left(\mathrm{CH}_{3}\right)_{3}$

4.15 Elemental mapping by EDX for droplet/cone CVD samples grown at $925{ }^{\circ} \mathrm{C}$ from $\mathrm{Ga}\left(\mathrm{CH}_{3}\right)_{3}$ and $\mathrm{N}\left(\mathrm{CH}_{3}\right)_{3}$ using $\mathrm{N} / \mathrm{Ga}=570 \ldots$

4.16 GIXRD of CVD sample grown at $925{ }^{\circ} \mathrm{C}$ from $\mathrm{Ga}\left(\mathrm{CH}_{3}\right)_{3}$ and

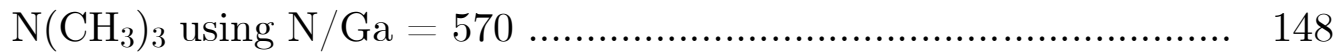

$5.1 \quad$ ORTEP drawing of 5 .................................................. 162

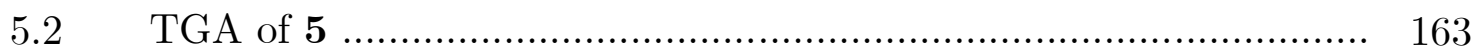

$5.3 \quad \mathrm{HOMO}$ and LUMO of 5 from DFT calculations ....................... 164

$5.4 \quad$ GPC for deposition of $\mathrm{InN}$ using 5 and $\mathrm{NH}_{3}$ plasma ................... 165

5.5 DFT calculated decomposition pathways and free energy profile for

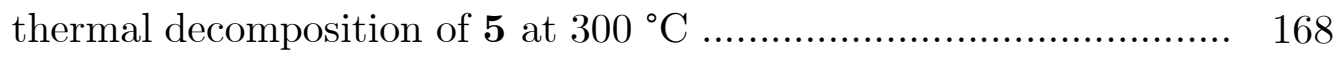

5.6 XRD, pole figure, SEM, and Raman measurements of InN deposited

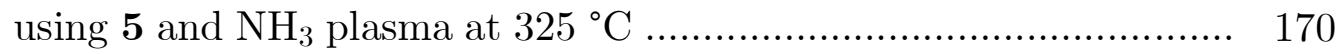

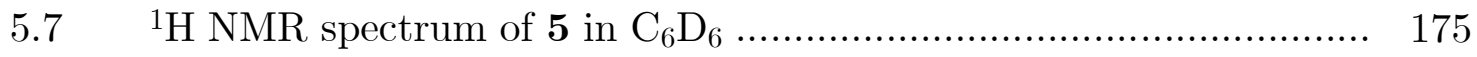

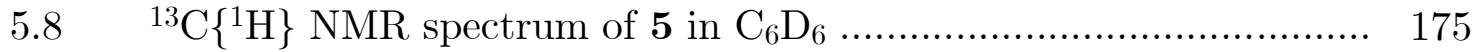

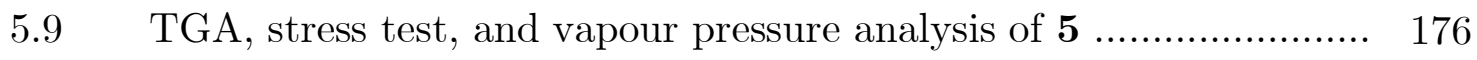

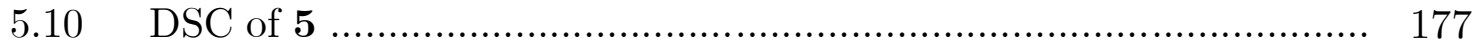

$5.11 \quad{ }^{1} \mathrm{H}$ NMR spectra of thermal decomposition study of $\mathbf{5}$................. 177

5.12 Enthalpy at $300{ }^{\circ} \mathrm{C}$ of decomposition paths of 5 ....................... 186

5.13 Growth behavior of $\mathbf{5}$ at $220{ }^{\circ} \mathrm{C}$ using $\mathbf{5}$ and $\mathrm{NH}_{3}$ plasma ............. 187

5.14 GIXRD of InN deposited at 200, 300, and $325{ }^{\circ} \mathrm{C}$ using $\mathbf{5}$ and $\mathrm{NH}_{3}$ plasma ................................................................... 188

5.15 Pole figure of $\mathrm{InN}$ deposited by $\mathbf{5}$ and $\mathrm{NH}_{3}$ plasma ................... 189

5.16 SEM of InN deposited at 220, 325, and $350{ }^{\circ} \mathrm{C}$ using $\mathbf{5}$ and $\mathrm{NH}_{3}$ plasma ................................................................... 190

5.17 HR-XPS for InN films deposited on $\mathrm{Si}(100)$ by $\mathbf{5}$ and $\mathrm{NH}_{3}$ plasma at $325{ }^{\circ} \mathrm{C}$

5.18 HR-XPS for InN deposited on $4 \mathrm{H}-\mathrm{SiC}$ by $\mathbf{5}$ and $\mathrm{NH}_{3}$ plasma at 325 ${ }^{\circ} \mathrm{C}$

5.19 Raman spectroscopy of $\mathrm{InN}$ from $\mathbf{5}$ and $\mathrm{NH}_{3}$ plasma at $300{ }^{\circ} \mathrm{C}$ on $\operatorname{Si}(100)$

5.20 General structure of a NacNac ligand .................................. 196

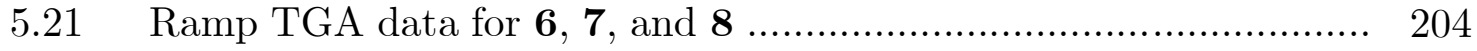

5.22 Crystal structure for oxo-bridged species obtained after exposing 6

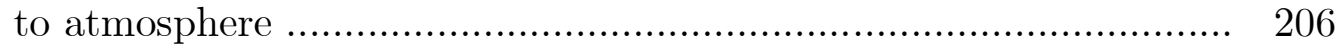

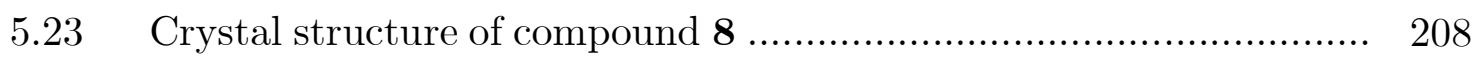

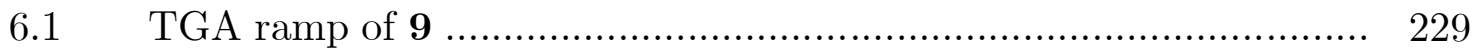


6.2 Saturation curve for $\mathbf{9}$ in ALD process with $\mathrm{NH}_{3}$ plasma, and SEM micrographs of AlN deposited ................................................. 230

6.3 GIXRD, $\sin ^{2} \psi$ vs. $\varepsilon$ plot, and XPS of AlN deposited using 9 and

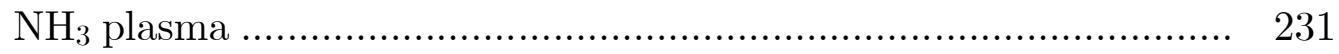




\section{List of Tables}

2.1 Relative electronic energy and free energy for stationary points in the deinsertion mechanism from $\mathbf{1}$ to $\mathbf{2}$ and from $\mathbf{2}$ to $\mathbf{3}$............. 40

$2.2 \quad$ Important bond lengths for 1-3 and intermediates ................... 53

$2.3 \quad$ Relative free energy of $\mathbf{1 - 3}$ and intermediates .......................... 53

3.1 ALD conditions for deposition of $\mathrm{Al}_{2} \mathrm{O}_{3}$ from TDMAA and water 61

3.2 ALD conditions for deposition of aluminum nitride using TDMAA and $\mathrm{N}_{2}$ plasma .......................................................... 61

4.1 Reaction enthalpies and free energies for unimolecular decomposition of ammonia and methylamines at CVD conditions $\left(300-1300{ }^{\circ} \mathrm{C}\right.$ and pressure 50 mbar) ....................................

4.2 Reaction enthalpies and free energies for bimolecular decomposition of ammonia and methylamines at CVD conditions

4.3 Activation enthalpies and free energies for bimolecular decomposition at CVD conditions ....................................

4.4 Reaction enthalpies and free energies for adduct formation between $\mathrm{NH}_{3-\mathrm{x}}\left(\mathrm{CH}_{3}\right)_{\mathrm{x}}$ and $\mathrm{Ga}\left(\mathrm{CH}_{3}\right)_{3}$ and subsequent alkane elimination reactions at $\mathrm{CVD}$ conditions

4.5 Activation enthalpies and free energies for alkane elimination from the adducts at CVD conditions

4.6 Reaction enthalpies and free energies for reaction of ammonia and methylamines with the H-terminated GaN (0001) surface at CVD conditions

4.7 Adduct formation between $\mathrm{NH}_{3-\mathrm{x}}\left(\mathrm{CH}_{3}\right)_{\mathrm{x}}$ and TMA, and subsequent hydrocarbon elimination reactions at CVD conditions

4.8 Surface interaction of ammonia and methylamines with GaN at CVD conditions

$5.1 \quad$ Crystal data and structure refinement for 5 .......................... 178

5.2 Atomic coordinates and equivalent isotropic displacement parameters for $\mathbf{5}$

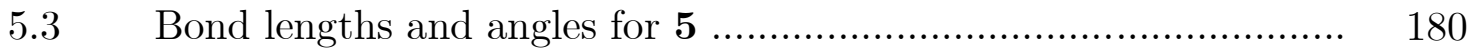

$5.4 \quad$ Anisotropic displacement parameters for 5 ......................... 184 
5.5 Hydrogen coordinates and isotropic displacement parameters for 5

5.6 Ligand dissociation enthalpy at STP and HOMO/LUMO energy levels for $\operatorname{In}(\text { famd })_{3}$ and $\mathbf{5}$

5.7 Natural bond orbital charges of $\mathrm{In}$ and bonding $\mathrm{N}$ atoms for $\operatorname{In}(\text { form })_{3}$ and $\mathbf{5}$

5.8 Enthalpy for decomposition paths of 5 at 1 bar ........................ 186

$5.9 \quad$ Free energies for decomposition paths of 5 at 1 bar ................... 186

5.10 Reaction enthalpy for decomposition paths of 5 to a tricoordinated In(III) species at 1 bar ........................................................ 187

5.11 Reaction free energies for decomposition paths of $\mathbf{5}$ to a

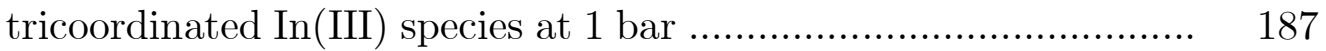

5.12 Crystal data and structure refinement for 8 .............................. 210

5.13 Atomic coordinates and equivalent isotropic displacement parameters for 8

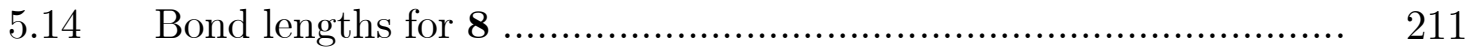

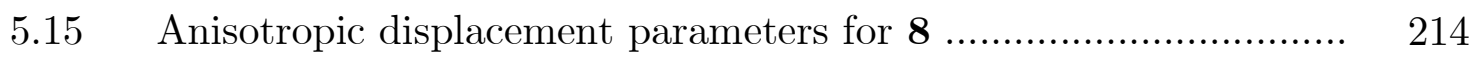

5.16 Hydrogen coordinates and isotropic displacement parameters for 8 .

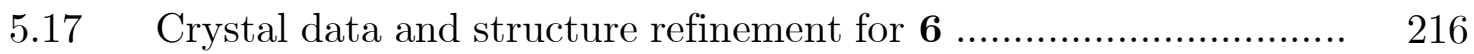

5.18 Atomic coordinates and equivalent isotropic displacement parameters for 6

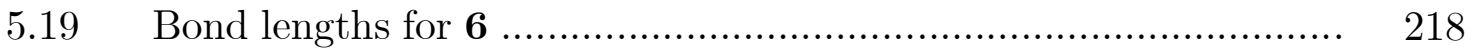

$5.20 \quad$ Anisotropic displacement parameters for 8 ............................ 221

5.21 Hydrogen coordinates and isotropic displacement parameters for 6 


\section{Acronyms}

\begin{tabular}{|c|c|}
\hline ALD & atomic layer deposition \\
\hline CB & conduction band \\
\hline CDI & carbodiimide \\
\hline CVD & chemical vapour deposition \\
\hline DFT & density functional theory \\
\hline DSC & differential scanning calorimetry \\
\hline EDX & energy-dispersive X-ray spectroscopy \\
\hline FWHM & full width at half maximum \\
\hline GIC & gas ionization chamber \\
\hline GIXRD & grazing-incidence X-ray diffractometry \\
\hline GPC & growth per cycle \\
\hline HAADF & high angle angular dark field \\
\hline HEMT & high electron mobility transistor \\
\hline HOMO & highest occupied molecular orbital \\
\hline LED & light-emitting diode \\
\hline LUMO & lowest unoccupied molecular orbital \\
\hline MBE & molecular beam epitaxy \\
\hline NMR & nuclear magnetic resonance spectroscopy \\
\hline PE-ALD & plasma-enhanced atomic layer deposition \\
\hline PVD & physical vapour deposition \\
\hline
\end{tabular}




$\begin{array}{ll}\text { RBS } & \text { Rutherford backscattering spectroscopy } \\ \text { SAED } & \text { selective area electron diffraction } \\ \text { SEM } & \text { scanning electron microscopy } \\ \text { STEM } & \text { scanning transmission electron microscopy } \\ \text { STP } & \text { standard temperature and pressure } \\ \text { TEG } & \text { triethylgallium } \\ \text { TEM } & \text { transmission electron microscopy } \\ \text { TGA } & \text { thermogravimetric analysis } \\ \text { TMA } & \text { trimethylaluminum } \\ \text { TMG } & \text { trimethylgallium } \\ \text { ToF-ERDA time of flight elastic recoil detection analysis } \\ \text { VB } & \text { valence band } \\ \text { VBM } & \text { valence band maxima } \\ \text { XPS } & \text { X-ray photoelectron spectroscopy } \\ \text { XRC } & \text { X-ray rocking curve } \\ \text { XRD } & \text { X-ray diffraction } \\ \text { XR } & \text { X-ray reflectivity } \\ \text { X } & \end{array}$




\section{Chapter 1}

\section{Introduction}

\subsection{Group 13 Nitrides}

Aluminum nitride, gallium nitride, and indium nitride (the so-called III-V semiconductors) have a large range of applications in microelectronics and other industries. These materials must often be deposited for use in devices in small and topographically non-trivial morphologies, which is why they are largely deposited by methods such as chemical vapour deposition (CVD) and atomic layer deposition (ALD) discussed further in Sections 1.2 and 1.4). Aluminum nitride will be principally discussed, compared to gallium and indium nitride, as it its deposition is central to the research presented in this thesis.

Aluminum nitride $(\mathrm{AlN})$ is a wide bandgap $(6.2 \mathrm{eV})$ semiconductor with a multitude of applications, including as a dielectric layer and for use in lightemitting diodes. ${ }^{1}$ It is also thermally conductive and has a high electrical resistivity 
$\left(10^{8}-10^{13} \Omega \mathrm{cm}\right) .^{2}$ AlN is self-passivating in high-oxidizing, high-temperature environments, and its inertness to chemical attack demonstrates its robustness for use in electronic devices. ${ }^{3}$ Gallium nitride is similarly mechanically, chemically, and thermally stable, and has a large direct bandgap $(3.43 \mathrm{eV}) .{ }^{4}$ Indium nitride has the smallest direct band gap of the 13-nitrides and a very large electronic mobility, as well as high peak and saturation velocities; ${ }^{5} \mathrm{InN}$ is the least studied of the 13 nitrides due strict temperature requirements for its deposition that prevent dissociation, as well as requiring a high N-In ratio. ${ }^{6}$ Importantly, the group 13nitrides can be deposited either in combination or sequentially to one another for use in high electron mobility transistors (HEMTs). ${ }^{7,8}$

CVD and ALD processes to deposit group 13 nitride films for electronics generally employ the trimethyl metal complexes as precursors with the general formula $\mathrm{M}\left(\mathrm{CH}_{3}\right)_{3}$, where $\mathrm{M}=\mathrm{Al}, \mathrm{Ga}$, or In, and most often use ammonia $\left(\mathrm{NH}_{3}\right)$ as a co-reagent. There are drawbacks to these processes, particularly the large ratio required for the nitrogen-containing precursor with respect to the metal-containing precursor; this is a significant problem for 13-N CVD processes. This is a reflection of non-ideal CVD chemistry, so the development of new 13-N processes (including precursor development) to deposit films by CVD and ALD is important, ongoing, and constitutes the bulk of the work of this thesis. 


\subsection{Vapour Phase Thin Film Deposition}

As advances in technology continue to evolve, the field of materials and deposition chemistry must advance as well to accommodate newly possible, and importantly, smaller physical features. Moore's Law predicts that every two years, the number of transistors on a dense integrated circuit will double, which effectively indicates that their size must be reduced. ${ }^{9}$ Fabrication techniques for these devices, therefore, must be chosen accordingly to be able to the control the growth of the necessary materials to build them.

As devices are being scaled down to the nanometer regime, thickness control at this level while also maintaining high control over growth and conformality is necessary; we must consider how nanofabrication can be used and modified to build these devices accordingly. The two main ways that nano-sized devices can be fabricated are by either top-down or bottom-up methods. A top-down synthesis begins with a larger bulk of material that will eventually become patterned on the

nanoscale, primarily by means of lithography. ${ }^{10}$ This thesis, however, will focus on thin film deposition techniques that are bottom-up, meaning that molecules are designed or chosen such that they will interact with one another to produce nanoscale structures (principally two-dimensional films in this work).

Several methods exist for depositing thin films of metals or ceramics, and choosing the appropriate method is of great importance since it could dictate film 
characteristics such as microstructure, surface morphology, or electrical and optical properties. ${ }^{11}$ Physical vapour deposition (PVD) techniques employ gaseous atoms or molecules (vapourized from a solid source) that condense on a substrate to form the desired film; this deposition occurs under partial vacuum. If this process occurs on the surface of a heated crystal, epitaxial films can be grown by molecular beam epitaxy (MBE), whereas high-energy ion bombardment can liberate atoms from target surfaces for sputtered depositions. ${ }^{12}$ Importantly, physical vapour techniques, as opposed to chemical vapour techniques, are line-of-sight techniques that limit the applications they can be used for. They are not suitable for substrates with complex morphologies, including those with trenches, vias, or other types of three-dimensional structures.

\subsubsection{Chemical Vapour Deposition}

Chemical vapour deposition (CVD) is a thin film deposition technique that employs gaseous precursors to deposit a desired material. Generally, a reactor chamber is heated and contains a substrate on which deposition is performed. One or more precursors are added to the system and can then react with one another in the gas phase and on the substrate surface to grow the desired film. CVD can be used to deposit a variety of films: specifically, metals, metal oxides, and metal nitrides are relevant with respect to this thesis. 
An important distinction between CVD and other PVD methods is that CVD precursors are volatilized and will ultimately undergo a chemical reaction at the substrate surface to produce a desired film. Gaseous precursors will be transported to the substrate and will react there to produce a solid film; these precursors will of course contain the elements necessary to deposit the desired material. ${ }^{13}$ The chemistry occurring at the surface determines the film growth, which is influenced by the chemical nature of the substrate itself, the chemical identities of the precursor(s), and their interactions with one another in the gas phase and at the surface. Notably, this growth method allows films to be deposited wherever surface chemistry happens, meaning that CVD is not only a line-of-sight technique. Physical characteristics of the deposited film will also be determined by these surface interactions, which can include a film's crystallinity, which can depend on the lattice matching between the substrate material and the desired film to be grown. ${ }^{14}$

To initiate film growth by CVD, the precursor(s) must be transported into the system to the substrate for deposition, usually by aid from an inert "carrier" gas. ${ }^{15}$ As this transport occurs, some precursors may undergo gas-phase decomposition reactions while others simply flow to the substrate. The deposition reaction may be a thermal decomposition, often involving organometallic precursors, while reductions using co-reagents (e.g., hydrogen gas) are also 
possible. ${ }^{13}$ Gas phase reactions can produce "secondary" precursors from those initially volatilised into the system, but importantly, these secondary precursors can also produce by-products that are not desired for the deposition of the required film. For this reason, it is important that by-products be volatile gases such that they can be purged out of and removed from the reaction chamber.

The precursor is ultimately transported to the surface and adsorbs; initial adsorption of precursors can be simply physisorption, after which diffusion or even desorption is possible, but will ultimately result in chemisorption of precursor molecules to the growth surface once a reaction occurs with a nucleation site. The desired material then continues to deposit in this fashion as precursors react to build the growing film and volatile by-products are removed from the reaction chamber.

Film thickness in CVD processes is a function of the exposure of precursors to the substrate, and a film's ultimate thickness is determined by the amount of time that surface reactions and growth can occur. Surface reactions will continue to occur between precursors as long as they are admitted to the reaction chamber; this is contrasted to atomic layer deposition which will be discussed below.

\subsubsection{Atomic Layer Deposition}

Atomic layer deposition (ALD) is another thin film growth technique that is a subset of CVD. The important distinction between these two processes is that 
CVD-grown films' thicknesses will increase with increased precursor exposure, while ALD-grown films undergo surface-limited, self-terminating reactions that do not allow for an increase in growth with increased precursor exposure after a critical exposure.

Precursors for ALD processes are also volatile species that are introduced to the reaction chamber, but this is done sequentially such that they do not encounter one another in the gas phase above the substrate. When the first precursor is introduced to the system, it will react with substrate nucleation sites, which can often be surface hydroxyls. Once the first precursor pulse is over, a monolayer is formed by the precursor molecule on the surface while remaining gaseous precursor and a volatile, gaseous by-product remains in the gas phase. Importantly, the precursor is not reactive with itself or the by-product, preventing any further chemical reaction beyond the formation of the monolayer. The by-product and any remaining gaseous precursor are then removed by a purge step with an inert gas such as nitrogen. The second precursor is then introduced into the reaction chamber and can react with the surface (which is now the monolayer formed on the substrate by the first precursor). Similarly, this second precursor is only reactive with the surface reactive sites, and not with itself or the by-products formed during this reaction step. A layer of the target film is formed, while additional gaseous precursor molecules are not reactive to this surface, and volatile by-products as 
well as any remaining precursor gas are removed in a second purge step. This cycle can then be repeated any number of times to achieve a film of desired thickness.

A growth process is deemed to exhibit ALD-type growth (instead of CVDtype growth) by producing what is referred to as a saturation curve. Since ALD growth is self-limiting, surface reactions are limited by precursor molecules chemisorbing with a limited number of nucleation sites, and not with themselves or other gaseous by-products. For this reason, once a sufficient dose of precursor is administered such that a complete monolayer is formed, any additional precursor present will not lead to an increase in film growth since no further chemical reactions can occur in the system. The growth per cycle (GPC) is measured ultimately by dividing total film thickness by number of cycles needed to produce it. In an ideal ALD process, GPC will increase linearly as a precursor is dosed to the system that reacts with an increasing number of available surface sites, up until a saturated monolayer is achieved (all available surface nucleation sites have reacted with a precursor molecule) and a GPC plateau is observed. The curve in Figure 1.1 demonstrates this behaviour as GPC increases linearly with precursor exposure up to a point of saturative growth; at this point, the GPC remains constant no matter how much the precursor dose is increased. We say that this growth is saturated and is exhibiting ALD-type behaviour. Contrarily, GPC values 
for CVD processes will continue to increase with increased precursor exposure since the surface reactions are not self-limiting.

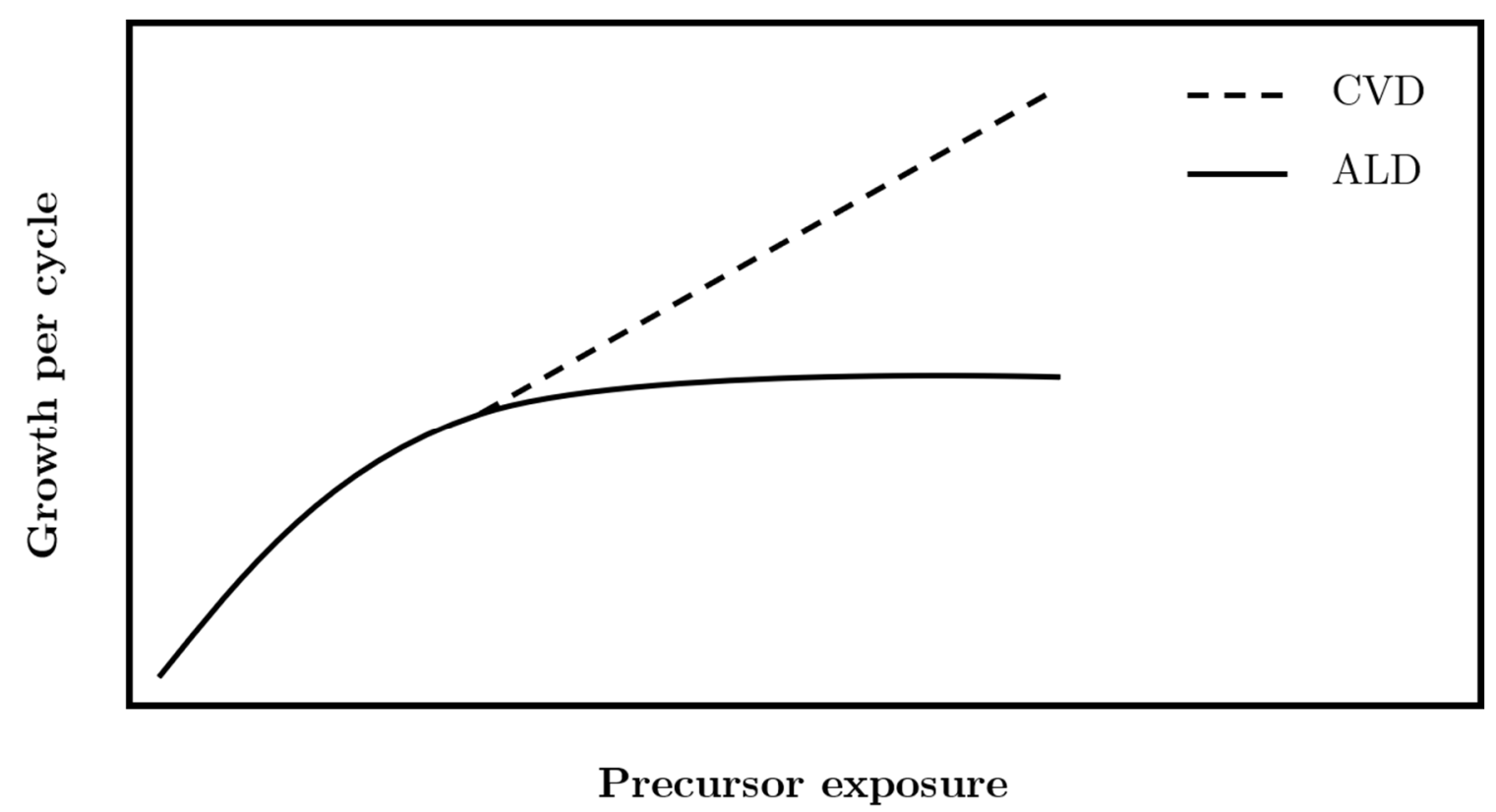

Figure 1.1: Generalized growth behaviour of ALD vs. CVD processes.

While ALD processes are often carried out thermally, plasma-enhanced ALD (PE-ALD) processes are also prevalent and have certain advantages. Principally, these processes can be carried out at lower temperatures which is beneficial or necessary to deposit on certain temperature-sensitive substrates. Thermal ALD processes rely on the substrate or reactor temperature to provide the necessary energy for surface reactions to occur, while PE-ALD processes have access to additional energy provided by the plasma itself. The plasma can contain ions, electrons, radicals, and light which can all supply energy to the ALD process and contribute to film growth; ions and electrons, specifically, have also been shown to 
modify the substrate which affects film growth characteristics. ${ }^{16}$ This surface modification typically results in increased nucleation, which allows increased growth of PE-ALD films. A direct plasma can be generated inside the deposition chamber itself by a power differential between the shower head and the stage, while a remote plasma is generated outside the deposition chamber and is electrically filtered so that only radicals are transported to the substrate for deposition reactions. ${ }^{17}$ The use of PE-ALD can be beneficial by allowing growth processes that are not accessible by thermal processes, like the reduction of certain metal precursors in order to deposit metal films. For example, while hydrogen gas is only reactive enough to reduce $\mathrm{TaCl}_{5}$ at over $400{ }^{\circ} \mathrm{C}$, highly reactive hydrogen radicals (formed in an $\mathrm{H}_{2}$ plasma) can produce a Ta film using this precursor below 200 ${ }^{\circ} \mathrm{C} .{ }^{18}$ Higher stability co-reagents such as $\mathrm{O}_{2}$ or $\mathrm{N}_{2}$ can also be made reactive enough to grow ALD films if employed in a plasma, and the densification of nitride films particularly is observed for PE-ALD compared to thermal ALD processes. ${ }^{17}$ While PE-ALD has many benefits over thermal ALD processes, some challenges are associated with it as well, particularly concerning reduced film conformality. This is particularly problematic on high-surface area substrates as well as those with high aspect ratio structures. ${ }^{19}$ While thermal ALD processes rely on selfterminating and limited surface reactions between chemical precursor sand surface sites, plasma processes employ other species, including radicals, that can react with a saturated monolayer and cause desorption from the surface. 


\subsection{Thermal Characterization of Precursors}

When a precursor is chosen or designed for use for ALD, it must first undergo thermal characterization to determine if its volatilization or decomposition behaviour will be appropriate for a self-limited growth process. The main tools we use for these characterizations are thermogravimetric analysis (TGA) and differential scanning calorimetry (DSC).

\subsubsection{Thermogravimetric Analysis}

Thermogravimetric analysis is an extremely beneficial tool for measuring the volatility of possible ALD precursors, and therefore their viability for use in an ALD process. For a precursor to be used in an ALD process, it must be volatile in a temperature window that is accessible on the tool being used; one can ascertain this volatility temperature using a simple TGA ramp experiment. In a TGA ramp, a small mass (approximately $10 \mathrm{mg}$ ) of precursor is placed on a tared aluminum pan on a hang-down wire. This is then enclosed by a furnace that will heat the sample, typically at a rate of $10{ }^{\circ} \mathrm{C}$ per minute. The sample's mass is measured while it is being heated, and a cleanly volatilizing sample will show a single exponential mass loss event as temperature increases. Ideally, this will lead to a remaining mass per cent of zero in the pan, indicating that all the sample volatilized from the pan and did not decompose or leave any impurities behind. The onset of 
volatility of the precursor is arbitrarily chosen as the temperature at which $95 \%$ of its original mass remains.

The derivative curve of this mass loss $(\mathrm{dm} / \mathrm{dt})$ will show any changes of slope that are indicative of decomposition events during volatilization, and the parent curve can sometimes to be used to determine decomposition products by the relative mass loss of ligands or moieties of those precursors. Figure 1.2 demonstrates both ideal and non-ideal TGA ramp curves.

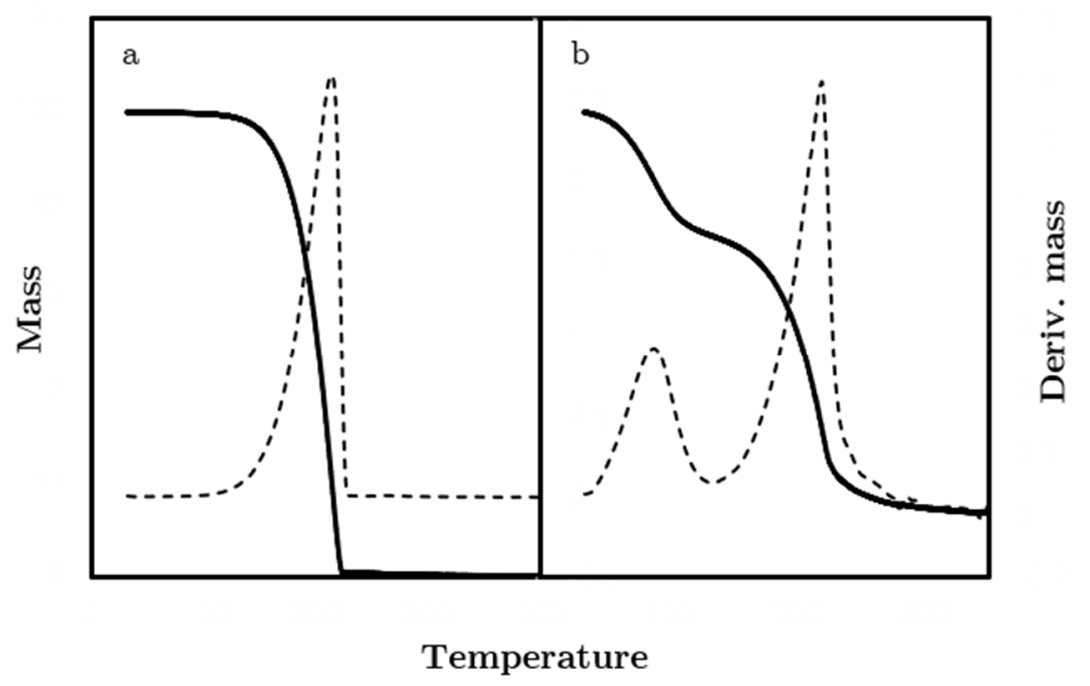

Figure 1.2: Thermogravimetric analysis traces of a compound demonstrating clean volatilization (a) and decomposition (b). The solid lines represent the sample mass as a function of temperature; an exponential mass loss in (a) indicates a clean volatilization which is supported by the $0 \%$ residual mass. Figure b shows two distinct features in the solid trace, which is indicative of two distinct thermal events, occurring due to decomposition. The dotted line indicates the derivative mass $(\mathrm{dm} / \mathrm{dt})$ of both curves and reflects the number of thermal events occurring during volatilization; figure (a) shows one feature while (b) demonstrates two features, indicative of decomposition of the initial precursor into another volatile species. 
Other important TGA experiments we typically employ are the isotherm and the stress test. In an isothermal experiment, a sample of precursor is held at a constant temperature for an extended period. If the temperature chosen allows for volatilization without decomposition, the mass of the precursor will decrease linearly over time to an ultimate mass of $0 \%$. If, however, the temperature chosen or is changed to one where precursor decomposition behaviour occurs, the slope of the isotherm will change, reflecting a new thermal event occurring. This could ultimately lead to a non-zero residual mass indicating that decomposition has occurred.

The stress test repeats the standard ramp experiment at increased mass loadings, typically 20 and $30 \mathrm{mg}$. As more precursor is loaded into the TGA pan, more of the sample will experience higher temperatures; if the residual mass is shown to remain constant (ideally at $0 \%$ ) with increased pan loading, the precursor is said to have good thermal stability. If, on the other hand, the residual mass increases, this is indicative of a high-temperature decomposition event that produces a non-volatile by-product.

Finally, TGA ramp data can be used to determine the 1 Torr temperature of potential ALD precursors. This is an important temperature, since 1 Torr of vapour pressure is a useful pressure for exposure in a deposition experiment. Derivative mass loss data $(\mathrm{dm} / \mathrm{dt}$ in units of $\mathrm{mg} / \mathrm{min}$ ) are converted to pressure data using 
the molecular weight of the precursor and the surface area of volatilization of the precursor. The natural logarithm of this pressure is then plotted as a function of reciprocal temperature in order to produce a Clausius-Clapeyron vapour pressure curve, which can be used to calculate the temperature at which the vapour pressure of the precursor is equal to 1 Torr. ${ }^{20}$

\subsubsection{Differential Scanning Calorimetry}

Differential scanning calorimetry (DSC) is also a useful technique for observing thermal behaviour of ALD precursors. A precursor sample between 0.200 and 0.500 mg is prepared in a sealed platinum pan, in addition to an empty sealed pan to be used as a blank. Both pans are placed on contacts in the DSC apparatus and as they are heated, the heat flow into or out of the precursor pan is measured with respect to the blank. The process measures and plots the amount of heat required to raise the temperature of the precursor pan with respect to the blank, which is dependent on the material and thermal processes occurring inside the sample pan. This method can be used to study phase transitions such as melting points, which will appear as endothermic events in the DSC curve. Exothermic processes can also be observed when less heat is required to maintain the temperature of the sample compared to the blank, and these can represent decomposition events. Exothermic decomposition events of course would be non-ideal in a potential ALD precursor, since this could lead to non-controlled growth during its use for film deposition; 
this can also determine between or below which temperatures ALD processes may be possible. The onset of decomposition of a precursor is arbitrarily chosen as a $5 \%$ increase in the exothermic peak observed. ${ }^{21}$

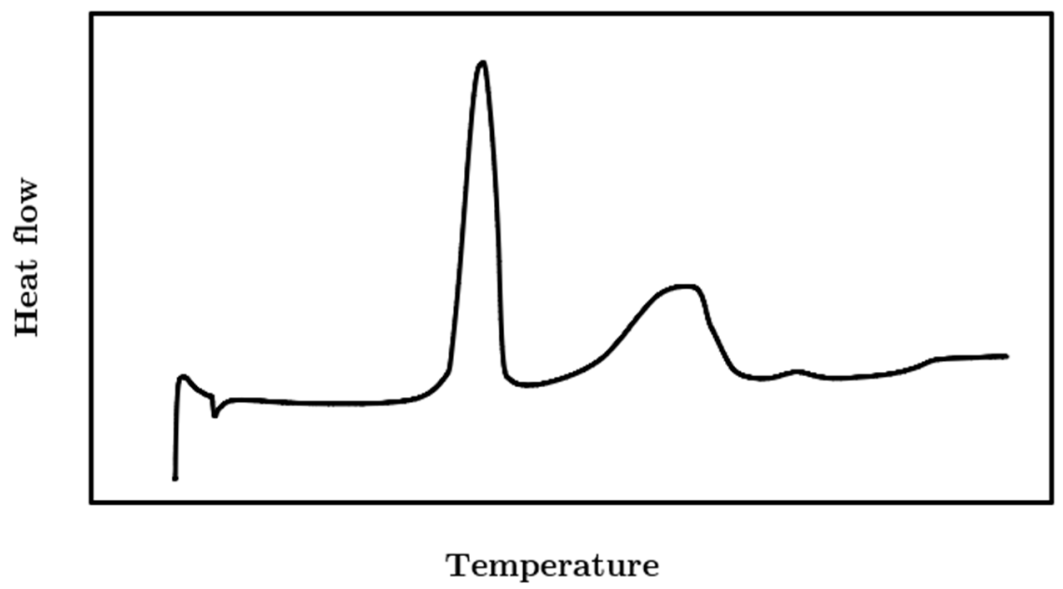

Figure 1.3: Differential scanning calorimetry trace of an ALD precursor. Negative (downward pointing) peaks are indicative of endothermic events, including melting point, while positive (upward pointing) peaks indicate exothermic events, which usually represent decomposition events.

\section{4 $\mathrm{Al}_{2} \mathrm{O}_{3}$ and $\mathrm{AlN}$ Precursors for ALD}

While there are a large variety of materials that can be deposited by ALD, aluminum oxide is likely the most deposited, most studied, and most wellunderstood. ${ }^{22}$ Aluminum oxide $\left(\mathrm{Al}_{2} \mathrm{O}_{3}\right)$ is a useful material to deposit for many reasons, principally for its use in microelectronics as a passivation layer or as a dielectric material. ${ }^{23}$ The use of aluminum oxide as a dielectric material and passivating layer is beneficial due to its large band gap (9 eV), high dielectric 
constant, as well as its high chemical and thermal stability. ${ }^{24}$ Several precursors exist that can be used to deposit aluminum oxide and aluminum nitride by ALD, ${ }^{25}$ though two of the most often used are aluminum trichloride $\left(\mathrm{AlCl}_{3}\right)$ and trimethylaluminum (TMA).

\subsubsection{Aluminum Trichloride}

Aluminum trichloride is inexpensive and accessible, is volatile when heated as a solid, and can be used as an ALD precursor for aluminum-containing films. This precursor was used as early as the 1970 s with water as a co-reagent to produce $\mathrm{Al}_{2} \mathrm{O}_{3}$ films by $\mathrm{ALD}^{26-28}$ and continued to be used into early 2000 s. ${ }^{29-31}$ It can also be reacted with oxygen gas as a co-reagent to produce aluminum oxide films. ${ }^{32,33}$ In addition to aluminum oxide, $\mathrm{AlCl}_{3}$ can also be used to deposit aluminum nitride (AlN) thermally by ALD using ammonia as a co-reagent, ${ }^{34,35}$ or in PE-ALD processes using $\mathrm{NH}_{3}+\mathrm{H}_{2}$ plasma as a co-reagent. ${ }^{36}$ The most common drawback of the use of $\mathrm{AlCl}_{3}$ as a precursor is the nearly inevitable incorporation of chlorine into deposited films, which can vary in concentrations of $<1 \%$ to $6 \%$ for $\mathrm{AlN}, 36,37$ as well as the production of $\mathrm{HCl}$ as a by-product; $\mathrm{HCl}$ can be detrimental to the deposition tool.

\subsubsection{Trimethylaluminum}

Trimethylaluminum (TMA) is by far the most studied ALD precursor, particularly in reference to its reactivity with water to produce aluminum oxide thin films by 
ALD. ${ }^{22}$ While $\mathrm{Al}_{2} \mathrm{O}_{3}$ films are largely prepared by the reaction of TMA and water, other co-reagents have also been employed including oxygen plasma ${ }^{38-40}$ and ozone. ${ }^{41-43}$

$$
\begin{array}{r}
\mathrm{Al}\left(\mathrm{CH}_{3}\right)_{3}(\mathrm{~g})+3 / 2 \mathrm{H}_{2} \mathrm{O}(\mathrm{g}) \longrightarrow{ }^{1} / 2 \mathrm{Al}_{2} \mathrm{O}_{3}(\mathrm{~s})+3 \mathrm{CH}_{4}(\mathrm{~g}) \\
\left\|\mathrm{OH}+\mathrm{Al}\left(\mathrm{CH}_{3}\right)_{3}(\mathrm{~g}) \longrightarrow\right\|-\mathrm{O}-\mathrm{Al}\left(\mathrm{CH}_{3}\right)_{2}+\mathrm{CH}_{4}(\mathrm{~g}) \\
\left\|-\mathrm{O}-\mathrm{Al}-\mathrm{CH}_{3}+\mathrm{H}_{2} \mathrm{O}(\mathrm{g}) \longrightarrow\right\|-\mathrm{O}-\mathrm{Al}-\mathrm{OH}+\mathrm{CH}_{4}(\mathrm{~g})
\end{array}
$$

Figure 1.4: Chemical reactions representing the overall stoichiometry of the ALD process to deposit aluminum oxide using water and TMA, followed by the "halfreactions" that represent the two chemical processes occurring during respective precursor pulses.

Several publications and reviews have been devoted to the study of the process to deposit $\mathrm{Al}_{2} \mathrm{O}_{3}$ using TMA and water by ALD. ${ }^{22,44}$ This process has been shown to occur by straightforward and self-limiting surface chemistry, which is the benchmark for any robust ALD process. Aluminum oxide is often deposited on silicon wafers, which are terminated with surface hydroxyl groups at a density of approximately $5-10 \mathrm{OH} / \mathrm{nm}^{2}{ }^{45}$ In the first step of deposition, TMA is exposed to the deposition chamber and will react with hydroxyl groups on the substrate surface, forming an oxygen-aluminum bond and producing methane $\left(\mathrm{CH}_{4}\right)$ gas as a by-product. TMA molecules are not reactive with themselves in the gas phase, nor are they reactive with chemisorbed $\|-\mathrm{O}-\mathrm{Al}-\mathrm{Me}_{2}$ surface moieties. The subsequent purge step removes any remaining TMA in the gas phase, as well as 
gaseous by-products. The second precursor, $\mathrm{H}_{2} \mathrm{O}$, is then exposed to the chamber and is reactive with the new monolayer that was formed during the previous precursor (TMA) pulse. Water will react with the surface methyl groups to ultimately generate a renewed hydroxyl monolayer. It will also produce gaseous byproducts that will be removed with any excess water during the second purge step. What is deposited is then ultimately a monolayer of $\mathrm{Al}_{2} \mathrm{O}_{3}$, and this process can be repeated (from the newly regenerated hydroxyl-terminated surface) to produce an alumina film of any desired thickness with Ångström-level precision.

Trimethylaluminum is also a very common precursor to use for the deposition of AlN by ALD. Thermal processes mostly used ammonia $\left(\mathrm{NH}_{3}\right)$ as a co-reagent up until around 2010; ${ }^{46-48}$ AlN ALD publications thereafter have largely employed plasma $\left(\mathrm{NH}_{3}, \mathrm{H}_{2} / \mathrm{N}_{2}\right.$, or a combination of both) as the co-reagent. ${ }^{49-51}$ For these processes, film quality is usually optimized for long plasma pulses, upwards of 30 seconds. $^{52}$ Since aluminum nitride is used as a semiconductor in microelectronics, its purity as a deposited film is crucial to consider; the most common impurities observed in AlN films are oxygen and carbon. Oxygen on the surface is expected since any AlN surface exposed to atmosphere will grow an alu- 


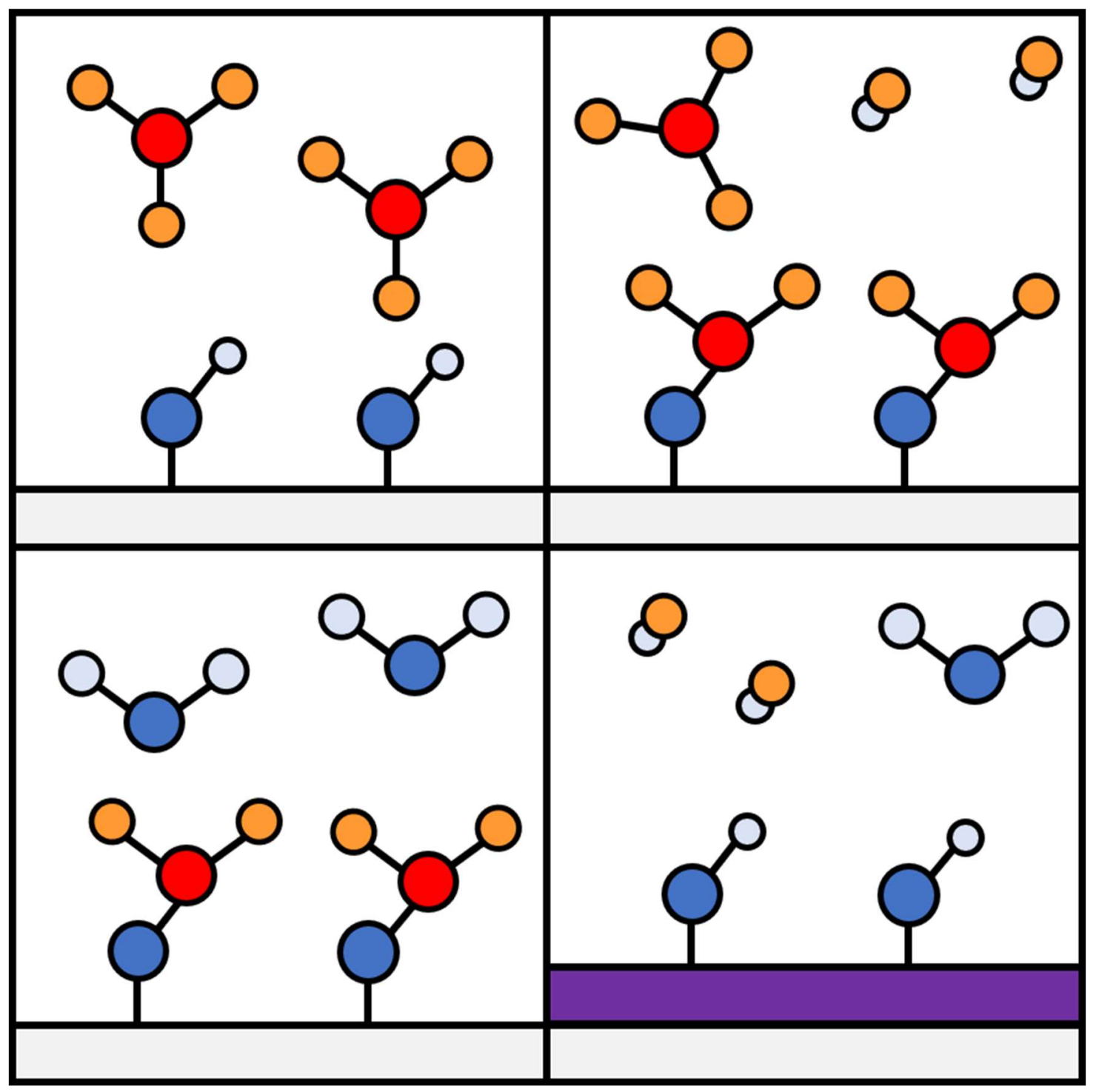

Figure 1.5: Process for the deposition of $\mathrm{Al}_{2} \mathrm{O}_{3}$ on a hydroxylated substrate by ALD using TMA and water. 
-minum oxide layer of approximately $5-10 \mathrm{~nm}$ thickness, ${ }^{53}$ though this oxygen typically does not penetrate into a high-quality film bulk. When using plasma as a co-reagent, however, it is important to consider the source since it has been demonstrated that using quartz ICP plasma sources can lead to higher levels of oxygen impurities in AlN films. ${ }^{54}$ Carbon impurities in AlN films deposited using TMA originate from a direct metal-carbon bond present in the precursor. ${ }^{37}$

\subsubsection{Amidinates and Guanidinates}

Precursors based on an amide framework are generally desirable for nitride ALD processes because they lack metal-carbon, metal-oxygen, and metal-halide bonds which can all lead to impurities in deposited films. Amidinates and guanidinates are popular and useful ALD ligands that have been used to deposit a variety of metals, metal oxides, and metal nitride thin films. ${ }^{55}$ They are easily modified by changing the functional groups on both the bonding nitrogen atoms and on the bridging carbon atom. Amidinates demonstrate pi-bonding resonance between the bonding sp2 nitrogen atoms, but this resonance can be expanded in guanidinates since the carbon substituent is an amido moiety that allows an additional imido resonance structure (Figure 1.6). ${ }^{55}$ This is crucial to consider when designing ALD precursors since the stability of the metal-ligand bond will determine its thermal properties, and ultimately, will allow (or not) the growth of films in a surfacecontrolled and self-limiting fashion. 


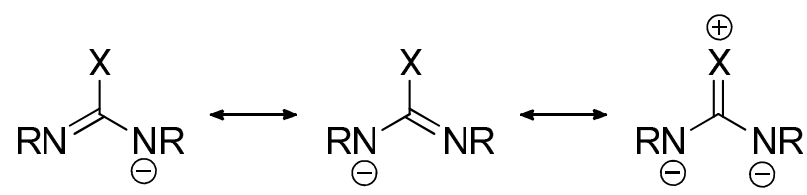

Figure 1.6: Resonance structures of amidinate and guanidinate ligands. For amidinates, $\mathrm{X}=\mathrm{R}, \mathrm{H}$ and only the first two resonance structures are possible. In guanidinates, all three resonances are possible since $\mathrm{X}=\mathrm{NR}_{2}{ }_{2} . \mathrm{R}$ and $\mathrm{R}^{\prime}$ can represent any alkyl species.

Amidinates and guandinates are easily synthesized, which is ideal if they are to be used as ALD precursors. Carbodiimides (CDI) can be inserted over both metal amides or metal alkyl species, though insertion into metal-amide bonds is more favourable since the amide nitrogen can donate a lone pair to form a coordinating, stabilizing bond with the metal centre. ${ }^{56}$

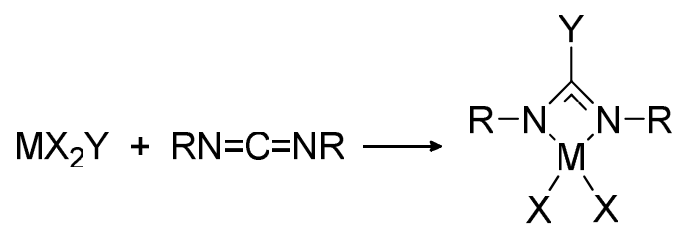

Figure 1.7: Insertion of a carbodiimide over a metal alkyl or amide species. The Y ligand represents an alkyl for amidinates and an amide for guanidinates, while $\mathrm{X}$ can be any other ligand. The carbodiimide $\mathrm{R}$ can be any alkyl species, but mostly represents ${ }^{i} \mathrm{Pr}$ for the work relevant to this thesis.

These ligands can be lithiated using simple alkyllithium reagents, and then reacted with metal chlorides (in appropriate stoichiometries) in order to form $\mathrm{MX}_{\mathrm{y}}(\text { guan } / \mathrm{amid})_{\mathrm{z}}(\mathrm{y}=1-3, \mathrm{z}=3-\mathrm{y})$ where $\mathrm{X}$ is a halide. The same precursors can also be synthesized by mixing protonated amidine or guanidine with metal trialkyls in a ligand-exchange reaction. ${ }^{57}$ 


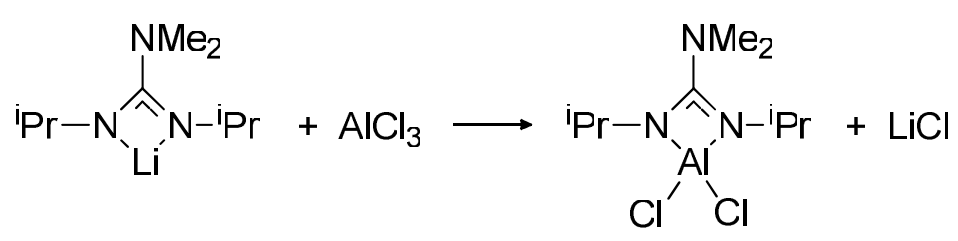

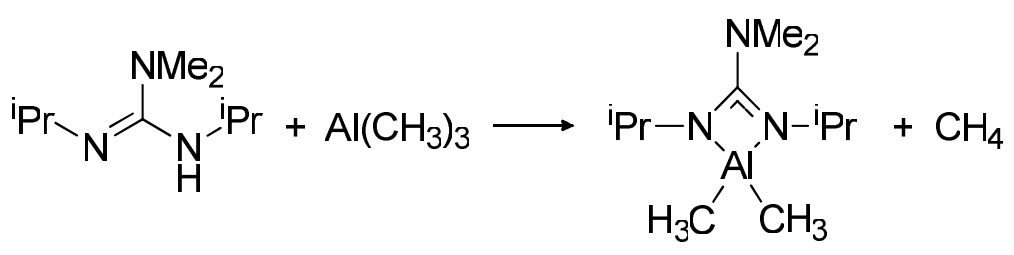

Figure 1.8: Reaction schemes demonstration the synthesis of an aluminum guanidinate precursor, either $\left[\left(\mathrm{Me}_{2} \mathrm{~N}\right) \mathrm{C}\left(\mathrm{N}^{\mathrm{i} P r}\right)_{2}\right]_{2} \mathrm{AlCl}_{2}$ in scheme a) or $\left[\left(\mathrm{Me}_{2} \mathrm{~N}\right) \mathrm{C}\left(\mathrm{NiPr}_{2}\right]_{2} \mathrm{Al}\left(\mathrm{CH}_{3}\right)_{2}\right.$ in scheme b). These syntheses occur by by a) salt metathesis between a lithiated guanidinate with aluminum trichloride and b) ligand exchange between a protonated guanidinine and trimethylaluminum.

Group 13 amidinates and guanidinates have been used to deposit metal oxides by ALD, including [MeC(NiPr)2]AlEt ${ }_{2}$ for the deposition of aluminum oxide at a very high growth rate by ALD using water as a co-reagent. ${ }^{58}$ Carbon contamination measured by Auger electron spectroscopy was $0.62 \%$, which can be attributed to the lack of metal-carbon bonds in the precursor. A gallium amidinate analogue has also been used to study its surface behaviour during growth on silica, ${ }^{59}$ as well as to grow gallium/gallium oxide nanostructures in a CVD experiment using water as a coreagent. 60

While amidinates and guanidinates are useful for some depositions, their main drawback as precursors is their thermal instability, namely that they undergo CDI de-insertion and are susceptible to beta-hydrogen elimination, and decompose at 
elevated temperatures. ${ }^{61}$ Upon heating, several aluminum amidinates have produced CDI that was condensed from the gas phase as a product in TGA experiments; ${ }^{62}$ this process is analogous for aluminum and other group 13 guanidinates. The study of a copper (I) amidinate species demonstrated that a second thermolysis pathway exists which leads to the production of free amidine at elevated temperatures, ${ }^{63}$ while a beta-hydrogen elimination pathway also led to the production of an oxidized guanidine (i.e., a secondary ketimine). A comparison between density functional theory (DFT) calculations and thermal experiments showed that CDI de-insertion decomposition pathways dominate at lower temperatures in gas or solution state, and beta-hydrogen elimination occurs mainly at higher temperatures in the gas phase. ${ }^{64}$ For these reasons, amidinate and guanidinate precursors are not necessarily ideal for use in large-scale ALD operations; their low thermal decomposition temperatures limit conditions under which depositions can happen and introduce uncertainty into the reactivity of the precursor at the surface. This thesis is concentrated on the design and use of simpler precursors using an amide framework, while still maintaining a lack of metal-carbon or metal-oxygen bonds, in order to deposit metal nitride films by ALD.

\subsubsection{Other Aluminum Precursors}

The most common aluminum precursors, TMA and $\mathrm{AlCl}_{3}$, have been discussed, as have amidinates and guanidinates which have been an extremely important class 
of ligands for the field of ALD and for group 13 compounds. The focus of this thesis is the innovation of new group 13 precursors, based on these frameworks which have been shown to be useful in the realm of group 13-based ALD.

TMA is an excellent starting point for a basis to build new precursors designed specifically for the deposition of aluminum nitride. The use of TMA in the deposition of aluminum oxide and nitride by ALD, however, can lead to the incorporation of carbon into films; this is due to the direct metal-carbon bonds found in the precursor. To deposit group 13 nitrides with low and controlled impurity levels, metal-nitrogen bonds in the precursor should be beneficial in reducing carbon incorporation into deposited films. The simplest derivative of TMA only employing metal-nitrogen bonds would be a homoleptic amide precursor $\left(\mathrm{M}\left(\mathrm{NR}_{2}\right)_{3}\right)$; substituent groups for this family of precursors could be easily manipulated as desired by simply changing starting synthetic materials, and choosing an appropriate $\mathrm{R}$ group will largely determine if the synthesized precursor will be viable for use in an ALD process. Small and lightweight $\mathrm{R}$ groups in this case would be desirable to use for ALD precursors to produce low molecular weight and volatile molecules, while bulky or heavy substituents should be avoided. Chapter 3 will discuss the use of a tris(dimethylamido)aluminum(III) precursor to deposit $\mathrm{Al}_{2} \mathrm{O}_{3}$ and aluminum oxy-nitride films by ALD. 
We can also consider building from ligand frameworks such as amidinates and guanidinates, since they have shown success in depositing group 13 compounds by ALD but have suffered in certain aspects such as thermal stability. From this, other bidentate ligands can be employed on group 13 metals, and particularly those that will only contain metal-nitrogen bonds. A ligand that is explored during this thesis is the MeNacNac ligand, a $\beta$-diketiminate, which will be discussed in more detail in Chapter 5. These NacNac ligands bear some similarly to another group of ligands, $\beta$-diketonates, that have been investigated for use in the deposition of various metal oxides by vapour-phase deposition techniques. In $\beta$-diketonates, both bonding nitrogen atoms in NacNacs are replaced by oxygen atoms. These ligands include acetylacetonate, which has been used to deposit cobalt oxide by CVD. ${ }^{65}$ The $\beta$-diketonate is an extremely useful and convenient ligand that can be purchased on its own or complexed to a metal centre as a precursor already, though it is not useful for the deposition of group 13 nitrides. Aluminum, particularly, is a very oxophilic element, so any oxygen present in an aluminum ALD precursor has a higher likelihood to be incorporated in deposited films. If one bonding oxygen atom in $\beta$-diketonates is replaced with nitrogen, $\beta$-ketoiminates are produced which have also been employed largely by the Devi group to deposit a variety of metal oxides. ${ }^{66}$ Again, however, these ligands are not suited to group 13 nitride deposition due to their direct metal-oxygen bonds. 
<smiles>[R][Y1]([R])[2H]</smiles><smiles></smiles><smiles>[Y][Y4]1oc([R])cc1[R]</smiles><smiles>[R2]N[Y10]([R2])N[R2]</smiles><smiles>[Y][Y]([Y])=[V]C(C)(C)C</smiles><smiles></smiles><smiles>[Y][Y10]1=C([R])C([R])=C([R])ON1[Y]</smiles><smiles>[R]C1=C([R])N([R])[Y4]([Y])([Y])N([R])C1[R]</smiles><smiles>[R20]N[Y]([R2])[H]</smiles><smiles>[R20][Y][H]</smiles>

c

Figure 1.9: Array of ALD precursors that are relevant to this thesis. M represents a group 13 metal (Al, Ga, In) and Y represents any other ligand. In row i), metal trialkyls (ex. TMA) and metal trihalides (ex. $\mathrm{AlCl}_{3}$ ) are shown. In row ii), figure iia) represents an amidinate precursor and iib) represents a guanidinate precursor, where $\mathrm{R}$ and $\mathrm{R}$ ' can be an alkyl species. In row iii) from figures iiia) through iiic) respectively, $\beta$-diketonates, $\beta$-ketoiminates, and $\beta$-diketiminates (NacNacs) are represented, respectively; R, R', and R" can be an alkyl species. Row iv) demonstrates a homoleptic metal amide in position iva), followed by two heteroleptic amide/hydride precursors in ivb) and ivc). All precursors are drawn as monomers for simplicity. 
Since aluminum is such an oxophilic element, an effort must be made to keep oxygen out of AlN ALD processes, even when using precursors that contain no oxygen atoms. Most ALD tools are loaded and unloaded under normal atmosphere, so films are exposed to oxygen and water before and after deposition; if grown films are of high quality, it is the hope that this oxygen does not penetrate into the AlN and will only form a 5-10 nm passivating layer on top. In order to minimize the chance of oxygen incorporation during film deposition, for example from residual oxygen or water in the ALD tool, the addition of hydride ligands was used in the design of a new ALD precursor; this will be further discussed in Chapter 6. If a reducing atmosphere could be present during the deposition of AlN itself, it is less likely that oxygen would incorporate into the film. Heteroleptic aluminum hydrides have not been reported for the deposition of AlN by ALD, though some publications using alane complexed with an amine as a precursor have been published. ${ }^{67} \mathrm{~A}$ thermally stable $\mathrm{Al}$ hydride complex has also been used to deposit aluminum metal by ALD. ${ }^{68}$ 


\section{Chapter 2}

\section{Thermal study of an indium trisguanidinate as}

\section{a possible indium nitride precursor}

Modified from the original manuscript published as:

Buttera, S. C.; Rönnby, K.; Pedersen, H.; Ojamäe, L.; Barry, S. T. Thermal Study of an Indium Trisguanidinate as a Possible Indium Nitride Precursor. J. Vac. Sci.

Technol. A Vacuum, Surfaces, Film. 2018, 36 (1), 01A101. https://doi.org/10.1116/1.5002634. 


\subsection{Abstract}

Tris-N,N,-dimethyl-N',N"- diisopropylguanidinatoindium(III) has been investigated both as a chemical vapor deposition precursor and an atomic layer deposition precursor. Although deposition was satisfactory in both cases, each report showed some anomalies in the thermal stability of this compound, war renting further investigation, which is reported herein. The compound was found to decompose to produce diisopropylcarbodiimide both by computational modeling and solution phase NMR characterization. The decomposition was shown to have an onset at approximately $120{ }^{\circ} \mathrm{C}$, and had a constant rate of decomposition from $150{ }^{\circ} \mathrm{C}$ to $180{ }^{\circ} \mathrm{C}$. The ultimate decomposition product was suspected to be bisdimethylamido-N,N,-dimethyl-N',N"- diisopropylguanidinato-indium(III), which appeared to be an intractable, nonvolatile polymer.

\section{$2.2 \quad$ Introduction}

Indium nitride ( $\mathrm{InN})$ is a small band-gap semiconductor with the largest electron saturation velocity, smallest effective electron mass and highest electron mobility among the group 13 nitrides. ${ }^{69-71}$ It is possible to integrate $\mathrm{InN}$ in existing high electron mobility transistors (HEMT) making InN a key material for future high frequency transistors. However, deposition of single crystalline $\mathrm{InN}$ films is challenging with conventional chemical vapor deposition (CVD) technology using 
trimethyl indium $\left(\operatorname{In}\left(\mathrm{CH}_{3}\right)_{3}\right)$ and ammonia. The $\mathrm{InN}$ film decomposes to indium metal and $\mathrm{N}_{2}$ gas even at deposition temperatures as low as $500{ }^{\circ} \mathrm{C},{ }^{72}$ setting an upper limit for the deposition; this forces the use of use $\mathrm{NH}_{3} / \mathrm{In}\left(\mathrm{CH}_{3}\right)_{3}$ ratios on the order of 100,000 due to the poor reactivity of ammonia at these low temperatures. ${ }^{6}$ An alternative approach for depositing $\mathrm{InN}$ is a time-resolved CVD technique where the poor reactivity of $\mathrm{NH}_{3}$ is overcome by allowing it to react with chemisorbed indium-containing surface moieties rather than relying on this reactivity in the (dilute) gas phase. Recent studies of $\mathrm{InN}$ deposition by atomic layer deposition (ALD) have shown that it is indeed possible to deposit InN with high crystalline quality using $\operatorname{In}\left(\mathrm{CH}_{3}\right)_{3}$ and nitrogen plasmas. ${ }^{73,74}$

For use in electronics, one of the most important aspects of the deposited InN film is low impurity levels, particularly for carbon and oxygen. It is a wellknown problem in ALD that the low deposition temperatures used to maintain selflimiting behaviour can lead to carbon impurities in the few atomic percent range when metal precursors with metal-carbon bonds are used. ${ }^{75}$ A recent study on plasma ALD of InN demonstrated that very long plasma exposures (on the order of $100 \mathrm{~s}$ ) were needed to bring the carbon levels in InN films down to around 3 atomic percent. ${ }^{74}$ Great care must also be taken in the construction and operation of the ALD reactor to minimize any traces of oxygen as indium is more prone to form the oxide, $\mathrm{In}_{2} \mathrm{O}_{3}$, than the nitride, InN. This is highlighted by the standard formation enthalpies $\left(\Delta \mathrm{H}_{\mathrm{f}}{ }^{\circ}\right)$ for $\operatorname{In}_{2} \mathrm{O}_{3}$ and $\mathrm{InN}\left(-925.8 \mathrm{~kJ} / \mathrm{mol}^{76}\right.$ and $-36.3 \mathrm{~kJ} / \mathrm{mol}^{77}$, 
respectively). Thus, InN ALD cannot be undertaken in an oxidizing atmosphere and chosen indium precursors suitable for ALD of $\operatorname{In}_{2} \mathrm{O}_{3}$ such as $\operatorname{In}(\mathrm{I}) \mathrm{Cp}$ cannot be used as there is nothing to oxidize the metal center to $\operatorname{In}(\mathrm{III})$.

When considering an ALD precursor for oxygen and carbon free deposition of indium nitride, the lack of oxygen and In-C bonds in the precursor is paramount, as is the +III oxidation state of the indium center. Tris-N,N,-dimethyl-N',N"diisopropylguanidinatoindium(III) (1) has previously been used as both a CVD precursor $^{78}$ as well as an ALD precursor ${ }^{79}$ for the deposition of indium oxide. This suggests that this precursor has an appropriate chemisorption chemistry to be further explored for nitride deposition. However, an in-depth reading of the principle literature on this compound suggests that it exhibits a complicated thermal behaviour that needs to be investigated further.

In a previous publication, $\mathbf{1}$ was seen to have an encouraging thermogravimetric (TG) trace, with the onset of volatility at approximately $60{ }^{\circ} \mathrm{C}$ and 85 mTorr of vapor pressure at $150{ }^{\circ} \mathrm{C} .78$ The residual mass was about $12 \%$, and appeared to deposit indium metal. Chemical vapour deposition occurred above $275{ }^{\circ} \mathrm{C}$ using air (i.e., $\mathrm{O}_{2}+\mathrm{H}_{2} \mathrm{O}$ from ambient conditions) and resulted in an amorphous film that could be annealed to crystalline, cubic indium oxide at 1125 ${ }^{\circ} \mathrm{C}$. The film was found to contain $1-2 \%$ of indium metal as deposited. 
The same compound was shown to exhibit ALD-type deposition in a welldefined thermal window from $230{ }^{\circ} \mathrm{C}-300{ }^{\circ} \mathrm{C},{ }^{80}$ which overlaps with the suggested CVD conditions. At a growth temperature of $240{ }^{\circ} \mathrm{C}$, the growth rate of $\operatorname{In}_{2} \mathrm{O}_{3}$ was found to plateau at $0.4-0.45 \AA$ /cycle at 4 seconds exposure, using water as the co-reactant. Although the errors bars are significant, the plateau has a slope that hints at a small CVD component. GI-XRD showed the deposited film to be crystalline above $180{ }^{\circ} \mathrm{C}$, and XPS was used to determine that the film had appropriate stoichiometry.

In both previous reports, the thermal chemistry of $\mathbf{1}$ was seen to be complex. It showed some evidence of carbodiimide (CDI) elimination at temperatures as low as $110{ }^{\circ} \mathrm{C}$ in reference 11 , but was reported as stable up to $160{ }^{\circ} \mathrm{C}$ in reference 12 . In both cases, complex thermal behaviour was suggested at higher temperatures, with diisopropylcarbodiimide reported as a potential decomposition product in both cases. This is expected, as aluminum guanidinates have previously shown carbodiimide elimination. The prime example of this was the monoguanidinatobisamidoaluminum(III) species which was seen to produce carbodiimide and homoleptic trisamidoaluminum(III) in the gas phase (Fig. 2.1).

The aluminum analogue of $\mathbf{1}$ was seen to undergo a low-temperature thermolysis at approximately $120{ }^{\circ} \mathrm{C}$ by $\mathrm{TG}$ analysis, showing mass losses corresponding to carbodiimide elimination, although the carbodiimide was only seen as a thermolysis product in the solution phase. ${ }^{81}$ This suggests that there 


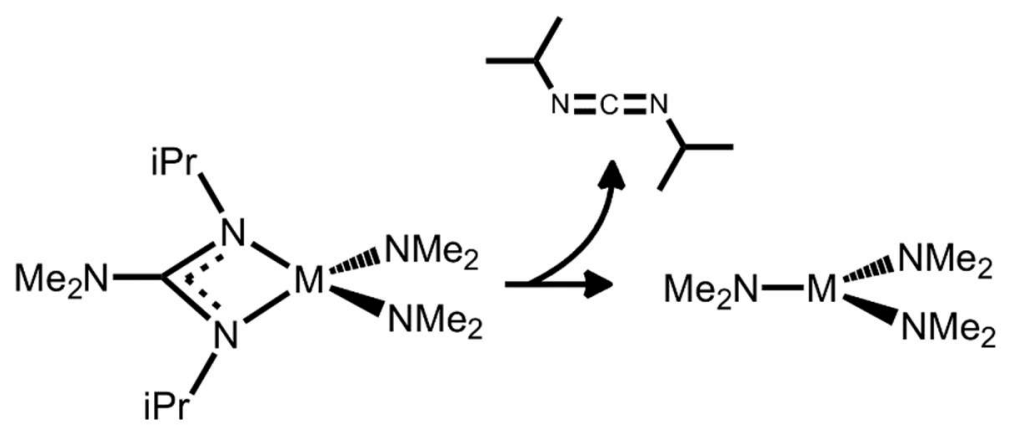

Figure 2.1: Elimination of diisopropylcarbodiimide from trisguanidinato group 13 compounds $(\mathrm{M}=\mathrm{Al}, \mathrm{Ga})$.

might be a thermal decomposition pathway for $\mathbf{1}$ that could produce a heteroleptic gas phase byproduct that acts as the deposition precursor (Fig. 2.2).

This is an important aspect of group 13 guanidinate chemistry to understand, since the ligand environment of the deposition precursor greatly affects the surface chemistry of deposition. A recent paper by Devi and coworkers shows that, compared to trisamidinatoindium (III) compounds, ${ }^{79}$ heteroleptic bisamidinatomonomethylindium(III) compounds have a higher residual mass in the TG (14\% vs. 5\%) and different crystallinity in the deposited indium oxide, suggesting different surface-precursor chemistry. Given that $\mathbf{1}$ was kept at $130{ }^{\circ} \mathrm{C}$ for $\mathrm{ALD}^{80}$ and $150{ }^{\circ} \mathrm{C}$ for $\mathrm{CVD},{ }^{78}$ the nature of this thermolysis needs to be better understood. Herein we report the synthesis of one of the intermediates of the carbodiimide elimination reaction, and discuss the thermolysis and likely fate of $\mathbf{1}$ when heated in a bubbler and in the gas phase. 


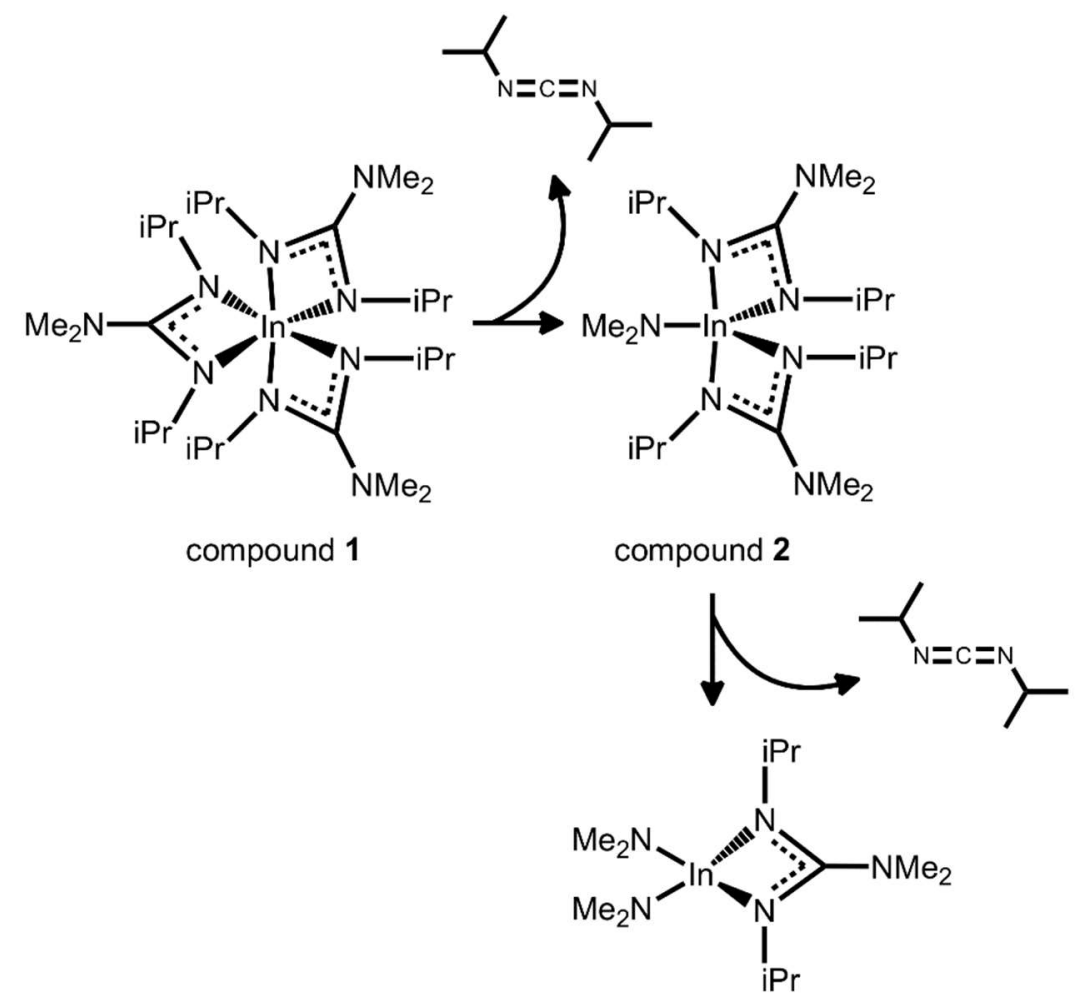

compound 3

Figure 2.2: Carbodiimide elimination to produce heteroleptic indium (III) species.

\subsection{Experimental}

\section{Precursor Synthesis}

\section{$\left[\left(\mathrm{Me}_{2} \mathrm{~N}\right) \mathrm{C}\left(\mathrm{N}^{\mathrm{i}} \mathrm{Pr}\right)_{2}\right]_{3} \mathrm{In}(1)$}

The synthesis of compound $\mathbf{1}$ was carried out in an MBraun Labmaster 300 nitrogen-filled glovebox. Firstly, a lithiated guanidinate species was synthesized by dissolving $\mathrm{LiNMe}_{2}(0.144 \mathrm{~g}, 2.7 \mathrm{mmol})$ in approximately $30 \mathrm{~mL}$ of hexanes and a few drops of ether in a 100-mL flask with rapid stirring. In a separate vial, N,N'diisopropylcarbodiimide $(0.345 \mathrm{~g}, 2.7 \mathrm{mmol})$ was dissolved in approximately $10 \mathrm{~mL}$ 
of hexanes. Both solutions were placed in the freezer for 20 minutes at $-35{ }^{\circ} \mathrm{C}$, then the carbodiimide solution was added dropwise by pipette to the $\mathrm{LiNMe}_{2}$ slurry. The mixture was allowed to warm to room temperature and was stirred overnight. The lithiated guanidinate product could not be isolated by drying, therefore, it was left in solution and used in the following synthetic step. ${ }^{1} \mathrm{H}$ NMR $\left(300 \mathrm{MHz}, \mathrm{C}_{6} \mathrm{D}_{6}\right)$ :

$\delta 3.74$ [sept, 2H, $\left.\mathrm{CH}\left(\mathrm{CH}_{3}\right)_{2}\right], 2.65$ [s, 6H, $\left.\mathrm{CN}\left(\mathrm{CH}_{3}\right)_{2}\right], 1.26$ [d, $12 \mathrm{H}, \mathrm{CH}\left(\mathrm{CH}_{3}\right)_{2}$ ]. Lithiated guanidinate $(3.002 \mathrm{~g}, 16.9 \mathrm{mmol})$ was dissolved in $50 \mathrm{~mL}$ hexanes and indium trichloride (1282 g, $5.8 \mathrm{mmol}$ ) was dissolved in $30 \mathrm{~mL}$ hexanes with rapid stirring. Both solutions were placed in the freezer, and the lithiated guanidinate solution was subsequently added to the indium trichloride solution dropwise by pipette. The $\mathrm{LiCl}$ salt was removed by filtration and the resulting solution was dried down and taken back up in hexanes and filtered again. The solvent volume was reduced by vacuum and the product was isolated by recrystallization as colourless crystals in a $-35{ }^{\circ} \mathrm{C}$ freezer $(2.542 \mathrm{~g}, 70.1 \%) .{ }^{1} \mathrm{H}$ NMR $\left(300 \mathrm{MHz}, \mathrm{C}_{6} \mathrm{D}_{6}\right)$ :

$\delta 3.78$ [sept, $\left.2 \mathrm{H}, \mathrm{CH}\left(\mathrm{CH}_{3}\right)_{2}\right], 2.67\left[\mathrm{~s}, 6 \mathrm{H}, \mathrm{CN}\left(\mathrm{CH}_{3}\right)_{2}\right], 1.50$ [d, $\left.6 \mathrm{H}, \mathrm{CH}\left(\mathrm{CH}_{3}\right)_{2}\right], 1.36$ [d, $6 \mathrm{H}, \mathrm{CH}\left(\mathrm{CH}_{3}\right)_{2}$ ].

\section{$\left[\left(\mathrm{Me}_{2} \mathrm{~N}\right) \mathrm{C}\left(\mathrm{N}^{\mathrm{i} P r}\right)_{2}\right]_{2} \mathrm{In}\left(\mathrm{NMe}_{2}\right)(2)$}

Compound $\mathbf{2}$ was prepared in an analogous manner as $\mathbf{1}$ substituting: lithiated guanidinate ( $5 \mathrm{~g}$ assumed, $28.2 \mathrm{mmol}$ ) in approximately $100 \mathrm{~mL}$ of hexanes, and indium trichloride $(3.122 \mathrm{~g}, 14.1 \mathrm{mmol})$ was dissolved in $80 \mathrm{~mL}$ tetrahydrofuran. 
The intermediate $\left[\left(\mathrm{Me}_{2} \mathrm{~N}\right)\left(\mathrm{N}^{\mathrm{i} P r}\right)_{2}\right]_{2} \mathrm{InCl}_{2}$ was isolated by recrystallization in hexanes (3.245 g, 46.9\%). ${ }^{1} \mathrm{H} \mathrm{NMR}\left(300 \mathrm{MHz}, \mathrm{C}_{6} \mathrm{D}_{6}\right)$ : $\delta 3.58$ [sept, $2 \mathrm{H}, \mathrm{CH}\left(\mathrm{CH}_{3}\right)_{2}$ ], $2.42\left[\mathrm{~s}, \quad 6 \mathrm{H}, \quad \mathrm{CN}\left(\mathrm{CH}_{3}\right)_{2}\right], \quad 1.34 \quad\left[\mathrm{~d}, \quad 12 \mathrm{H}, \quad \mathrm{CH}\left(\mathrm{CH}_{3}\right)_{2}\right] . \quad$ This intermediate $\left[\left(\mathrm{Me}_{2} \mathrm{~N}\right)\left(\mathrm{N}^{\mathrm{P} P r}\right)_{2}\right]_{2} \mathrm{InCl}_{2}$ was dissolved quantitatively in $40 \mathrm{~mL}$ ether and stirred to dissolve while lithium dimethylamide $(0.340 \mathrm{~g}, 6.6 \mathrm{mmol})$ was dissolved in $40 \mathrm{~mL}$ ether; the two solutions were placed in the $-35{ }^{\circ} \mathrm{C}$ freezer for 20 minutes after which the $\mathrm{LiNMe}_{2}$ solution was added to the $\left[\left(\mathrm{Me}_{2} \mathrm{~N}\right)\left(\mathrm{N}^{\mathrm{i} P r}\right)_{2}\right]_{2} \mathrm{InCl}_{2}$ solution dropwise by pipette. Compound $\mathbf{2}$ was obtained as a viscous yellow liquid $(1.096 \mathrm{~g}, 33.2 \%) .{ }^{1} \mathrm{H}$ NMR $\left(300 \mathrm{MHz}, \mathrm{C}_{6} \mathrm{D}_{6}\right): \delta 3.61$ [sept, $2 \mathrm{H}, \mathrm{CH}\left(\mathrm{CH}_{3}\right)_{2}$ ], 3.20 [s, 6H, $\mathrm{InN}\left(\mathrm{CH}_{3}\right)_{2}$ ], 2.53 $\left[\mathrm{s}, 6 \mathrm{H}, \mathrm{CN}\left(\mathrm{CH}_{3}\right)_{2}\right], 1.30$ [d, 6H, $\left.\mathrm{CH}\left(\mathrm{CH}_{3}\right)_{2}\right]$.

\section{Thermogravimetric Analysis}

For all TGA experiments, the same sequence of steps was followed on the TGA Q500 instrument inside a nitrogen-filled glovebox. The desired sample (between 10 and $30 \mathrm{mg}$ ) was placed on a tared platinum pan with nitrogen flow rates set to 60 sccm.

In ramped TGA experiments, the sample was placed on the pan and the furnace was heated at a rate of $10{ }^{\circ} \mathrm{C} /$ minute to $600{ }^{\circ} \mathrm{C}$. The mass loss profile for 1 showed a relatively clean volatilization curve without any obvious bumps indicative of decomposition, but had a residual mass of $16 \%$ (indicating some form of decomposition, possibly occurring simultaneously with volatilization). 
Compound 2 had a mass loss profile with various features indicative of decomposition and a residual mass of approximately $25 \%$.

Isothermal TGA experiments allowed the sample to be held at a constant temperature over the length of the experiment; the temperature chosen was roughly the temperature at which the ramp experiment demonstrated 1\% mass loss of the compound. In its isotherm at $130{ }^{\circ} \mathrm{C}$, compound 1 showed linear mass loss over time, but even after 1600 minutes of heating, the compound did not volatilize completely ( $70 \%$ mass remaining). At $150{ }^{\circ} \mathrm{C}$, the sample demonstrated a linear mass loss over time, until bottoming out at approximately $20 \%$ mass; this decomposition occurred after 200 minutes at $160{ }^{\circ} \mathrm{C}$.

\section{NMR Tube Thermolysis}

In the NMR tube thermolysis experiment of $\mathbf{1}$, a traditional NMR thermolysis was performed. A thick-walled NMR tube was filled with a sample of $\mathbf{1}$ inside the nitrogen glovebox. The sample was then dissolved in $\mathrm{d}_{6}$-benzene under an atmosphere of nitrogen on a Schlenk line. The NMR tube was flame sealed under vacuum on a Schlenk line after being under at atmosphere of argon gas. For the length of the experiment, the NMR tube was placed in a closed oven set at $150{ }^{\circ} \mathrm{C}$.

After 1 hour at $150{ }^{\circ} \mathrm{C}$, an ${ }^{1} \mathrm{H}$ NMR showed, aside from the expected peaks for 1, additional small peaks for N,N'-diiosopropylcarbodiimide (CDI) and protonated guanidine. The septets for both decomposition products (CDI and 
protonated guanidine) were overlapping, so their collective integration was calculated and compared to that of $\mathbf{1}$; note that these comparisons take into account that one molecule of precursor would decompose to three of either products. It is also important to note that over the course of these experiments, a brown insoluble product formed and was visible in the tube; due to its insolubility it was likely not represented in the NMR spectrum and its presence increased with increased heating and time.

After the first hour of heating at $150{ }^{\circ} \mathrm{C}$, the ratio of $\mathbf{1}$ to its decomposition products was approximately 4.7. Over a period of five hours, the decomposition products' appearance increased to raise the former ratio to 3.7. After a further 43 hours of heating at $150{ }^{\circ} \mathrm{C}$, the ratio only further increased to 3.3 . Over all time that 1 was heated at $150{ }^{\circ} \mathrm{C}$, the ratio of CDI to guanidine in the NMR spectrum remained relatively constant, decreasing slightly from 1.6 to 1.4 . The ratio of $\mathbf{1}$ to CDI decreased from $\sim 6.2$ to 4.6 , and the ratio of 1 to guanidine from 10.0 to 6.4. This is to say that $\mathbf{1}$ was certainly decomposing over time at $150{ }^{\circ} \mathrm{C}$, and decomposition to CDI was much more prevalent than to guanidine.

The temperature of the oven was then increased to $160{ }^{\circ} \mathrm{C}$. Over the first five hours at this temperature, the ratio of $\mathbf{1}$ to decomposition products remained constant around 3.3. The tube was then left to heat over time periods of 24 hours or greater; after 167 hours at $160{ }^{\circ} \mathrm{C}$ (on top of the 48 hours at $150{ }^{\circ} \mathrm{C}$ ), the ratio 
of $\mathbf{1}$ to decomposition products had increased to 1.93. The ratio of CDI to guanidine increased to 1.3 .

The NMR tube was subsequently heated at $170{ }^{\circ} \mathrm{C}$. After 24 hours, the ratio of precursor to decomposed product increased to 1.68, and after a further 72 hours, the ratio increased to $\sim 1.1$; the ratio of $\mathrm{CDI}$ to guanidine remained relatively constant from previous heating. The ratio continued to decrease over the next 24 hours to just below 1.

Upon increasing the temperature to $180{ }^{\circ} \mathrm{C}$ for 24 hours, the ratio of precursor to decomposed products decreased to 0.7 with the CDI:guanidine ratio constant around 1.3. After a final 24 hours of heating at $180{ }^{\circ} \mathrm{C}$, the ratio of $\mathbf{1}$ to decomposition products increased to 0.5 and the ratio of CDI to guanidine varied slightly to 1.1 .

\section{Modelling}

All quantum chemical computations were made with the Gaussian 09 software. ${ }^{82}$ Geometry optimization and frequency calculations were performed with the hybrid DFT functional B3LYP 83,84 and with $6-31+\mathrm{G}(\mathrm{d}, \mathrm{p})$ basis for lighter atoms and Los Almos double-zeta basis with associated effective core potential (lanl2dz) for indium. The method and basis have earlier been shown to give accurate values for structures of indium compounds. ${ }^{85}$ The geometries were verified to have none 
imaginary frequencies for the minima and one negative frequency along the reaction path for the transition states (TS).

\subsection{Results and Discussion}

The computational elimination mechanism was modeled as the reverse CDI insertion mechanism onto aluminum reported earlier, ${ }^{56}$ and similar stationary structures were found along the predicted reaction path. The mechanism is composed of six stationary points: compound $\mathbf{1}$, a chelation transition state (TS1), a dechelated intermediate, a migration transition state (TS2), adduct, and finally separated compound $\mathbf{2}$ and CDI (Fig. 2.3). The relative electronic and free energies for the different stationary structures are given in Table 2.1, while geometries with key bond lengths are given as supplementary material.

Table 2.1: Relative electronic energy and free energy for the stationary points in the deinsertion mechanism from 1 to $\mathbf{2}$ (1st) and from $\mathbf{2}$ to $\mathbf{3}$ (2nd). Energy given in $\mathrm{kJ} / \mathrm{mol}$.

\begin{tabular}{cccccccc}
\hline & & Reactant & TS1 & Intermediate & TS2 & Adduct & Separated \\
\hline First & $\Delta \mathrm{E}$ & 0.0 & 142.3 & 40.0 & 169.1 & 137.3 & 152.0 \\
& $\Delta \mathrm{G}(\mathrm{STP})$ & 0.0 & 132.0 & 33.4 & 148.8 & 110.6 & 52.4 \\
& $\Delta \mathrm{G}\left(240{ }^{\circ} \mathrm{C}\right)$ & 0.0 & 129.2 & 29.1 & 141.1 & 95.0 & -8.4 \\
Second & $\Delta \mathrm{E}$ & 0.0 & & & & & 150.0 \\
& $\Delta \mathrm{G}(\mathrm{STP})$ & 0.0 & & & & & 61.5 \\
& $\Delta \mathrm{G}\left(240^{\circ} \mathrm{C}\right)$ & 0.0 & & & & & 6.2 \\
\hline
\end{tabular}




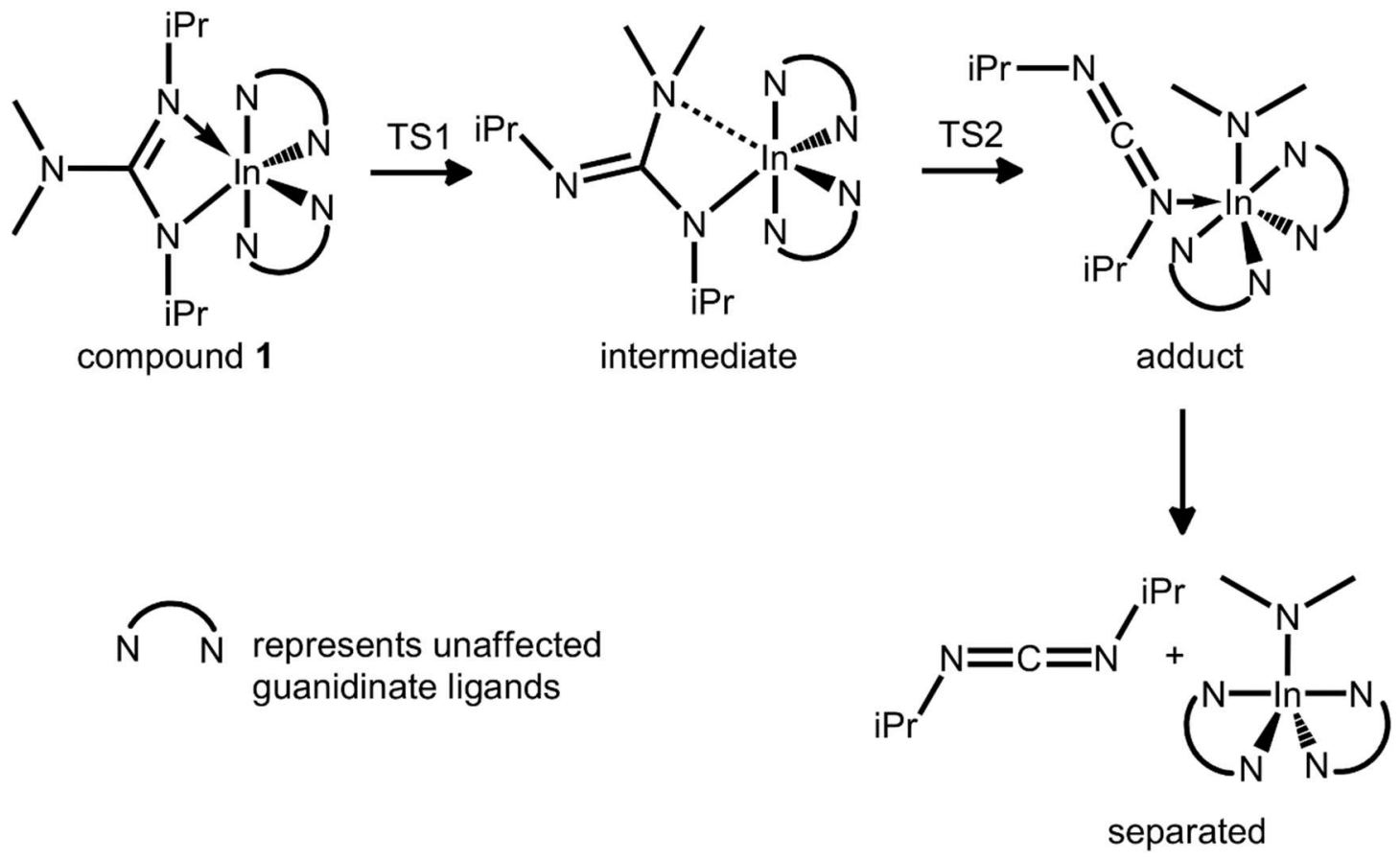

Figure 2.3: Proposed elimination reaction of CDI from 1. Note that only the guanidinate affected by the elimination is shown, for simplicity.

Compound $\mathbf{1}$ - In undecomposed $\mathbf{1}$ the guanidinate ligands were positioned symmetrically around a $\mathrm{C}_{3}$-axis. The indium nitrogen bonds showed a mixture of a coordinating and a covalent bonding resonating at the guanidinate core $(2.24 \AA)$. The bulky isopropyl groups caused the structure to become a densely packed ball covering the indium atom. The structure is similar to the previously obtained crystal structure. ${ }^{78}$

Chelation (TS1) - During the chelation one of the formamidine nitrogen detaches from the indium and rotates away while the dimethylamine nitrogen rotates toward the indium atom. Both nitrogen are approximately at the same 
distance from the indium center $(3.58 \AA$ respectively $3.77 \AA)$. The remaining formamidine nitrogen is drawn closer to the indium atom forming a covalent bond $(2.06 \AA)$.

Intermediate - The metastable intermediate was formed after further rotation allowing the lone pair on the dimethyl amine to coordinate to the indium center. As the structure is sterically relaxed the covalent nitrogen indium bond is somewhat elongated $(2.11 \AA)$. The migration barrier is slightly higher than the barrier for chelation in terms of electronic energy making it more probable for the intermediate to rechelate rather than migrate at low temperatures. The barrier difference for free energy is smaller and decreases with increased temperature indicating an equal probability for the reactions at higher temperatures.

Migration (TS2) - During the migration, the dimethylamine nitrogen approached the indium center to form a covalent bond instead of coordinating (2.59 vs. $2.11 \AA$ ) while the previously covalent bond between the formamidine nitrogen and indium elongates to a coordinating bond (from 2.11 to $2.49 \AA$ ). At the same time, the bond between the dimethylamine nitrogen and the central carbon elongated and severed, while a second double bond was formed between the carbon and the amidine coordinating to the indium.

Adduct - The adduct had significantly higher electronic energy and enthalpy than $\mathbf{1}$ and the intermediate. Steric hindrance between the isopropyl groups on the 
$\mathrm{CDI}$ and the other guanidinates forced the CDI from the indium atom making a very weak coordinating bond (3.97 $\AA$ ).

Separated - Separation of the adduct caused a slight increase in the electronic energy and enthalpy, but entropic factors permitted a significant drop in the free energy. As the temperature was increased in the model, the free energy decreased further. At standard temperature and pressure, $\mathbf{1}$ had the lowest energy, but at approximately $210{ }^{\circ} \mathrm{C}$ the separated products became lower in free energy. This confirms that the cause of CDI elimination is an increase in entropy.

Second elimination - Reactant and product geometries for the elimination of CDI from $\mathbf{2}$ to $\mathbf{3}$ was also computed. The resulting energies were similar to the first, with the main difference being that the relative free energy of the second elimination was slightly higher $(10-20 \mathrm{~kJ} / \mathrm{mol})$ for all investigated temperatures.

Temperature dependence - The reaction free energy for both eliminations was decreased with increased temperature, as was expected for an entropically driven process (Fig. 2.4). The first elimination changed from endo- to exergonic at approximately $210{ }^{\circ} \mathrm{C}$ while the second changed at approximately $265{ }^{\circ} \mathrm{C}$. The double elimination (the first followed by the second) also followed this trend but had a much steeper slope than single eliminations. The double elimination changed from endo- to exergonic at approximately $235{ }^{\circ} \mathrm{C}$. 


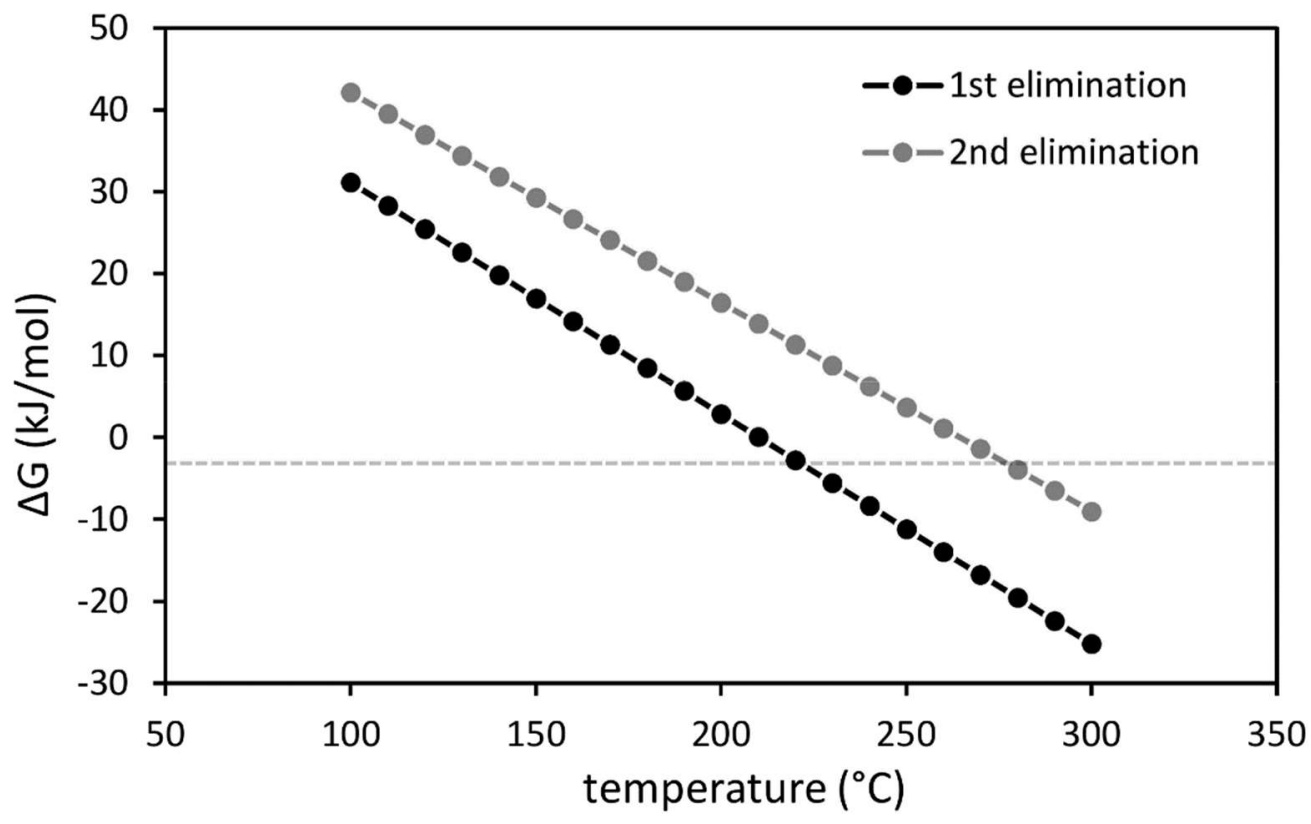

Figure 2.4: Temperature dependence of CDI elimination from $\mathbf{1}$ to $\mathbf{2}\left(1^{\text {st }}\right.$ elimination) and from 2 to 3 ( $2^{\text {nd }}$ elimination).

Equilibrium - From the reaction free energy, the equilibrium constant and the equilibrium concentration of the species in the reaction could be calculated. When the partial pressure of $\mathbf{1}$ was set to 1 mbar (a reasonable vapour pressure for deposition experiments), it showed some decomposition even at room temperature, and at temperatures above $160{ }^{\circ} \mathrm{C}$ it was almost fully decomposed (Fig. 2.5). The concentration profile shows similarities with the TG profile (vide infra). Compounds $\mathbf{1}$ and $\mathbf{2}$ were in a balanced equilibrium at about $120{ }^{\circ} \mathrm{C}$, and compound 3 began to show an appreciable partial pressure at this temperature. At the temperature of the vaporization processes $\left(130{ }^{\circ} \mathrm{C}-150{ }^{\circ} \mathrm{C}\right)$, both deinsertions could occur readily to produce $\mathbf{3}$. 


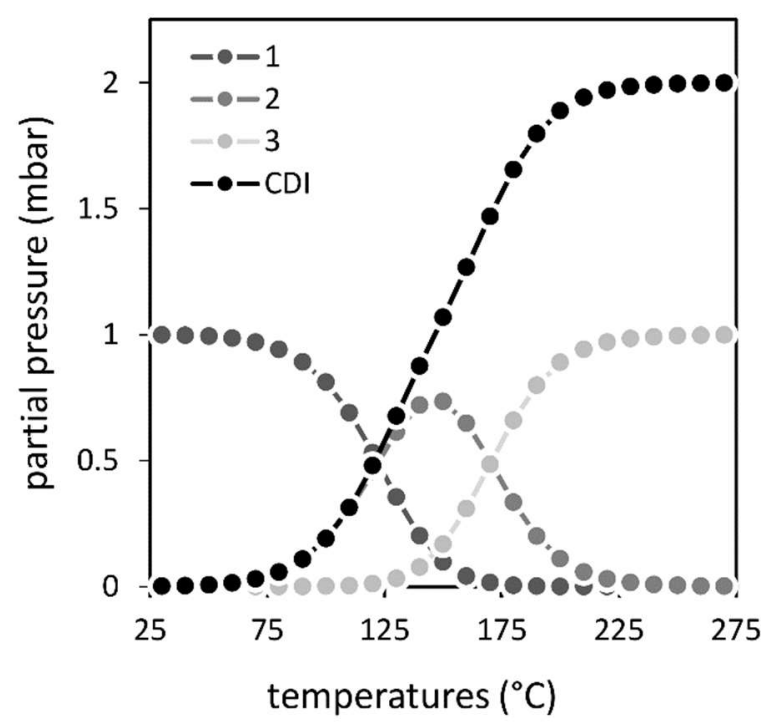

Figure 2.5: The partial pressures from CDI elimination of $\mathbf{1}$ to produce $\mathbf{2}$ and $\mathbf{3}$.

Thermolysis testing - Given the calculational conclusion that carbodiimide elimination is viable, an extended thermolysis testing was undertaken. In the literature, isotherms at $100{ }^{\circ} \mathrm{C}$ and $120{ }^{\circ} \mathrm{C}$ were reported to be linear over 3.5 hours, ${ }^{80}$ and extended 8-hour isotherms of $\mathbf{1}$ showed similar behaviour (Fig. 2.6).

The isotherms remained linear during mass loss at both $130{ }^{\circ} \mathrm{C}$ and $150{ }^{\circ} \mathrm{C}$ (N. B., the slight modulation at the top of each trace was due to ramping the TG to the set point temperatures). The isotherm at $130{ }^{\circ} \mathrm{C}$ showed a modest 0.013 $\mathrm{mg} /$ minute weight loss while the $150{ }^{\circ} \mathrm{C}$ showed $0.051 \mathrm{mg} /$ minute loss. The isotherm at $130{ }^{\circ} \mathrm{C}$ could not reasonably be run to completion since it would require a greater than 1600 minute experiment, but at $150{ }^{\circ} \mathrm{C}$, the linear weight loss had a $20 \%$ residual mass. This means that decomposition to a non-volatile side product 


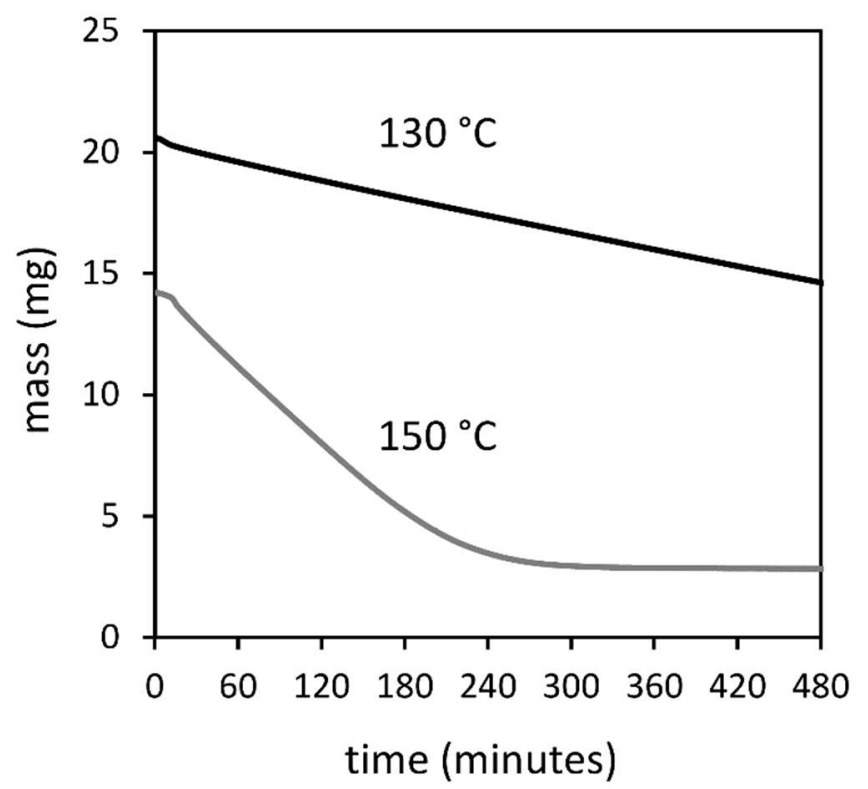

Figure 2.6: Isotherms of $\mathbf{1}$ at $130{ }^{\circ} \mathrm{C}$ (black) and $150{ }^{\circ} \mathrm{C}$ (grey) over eight hours. and evaporation occurred at the same rate throughout the $150{ }^{\circ} \mathrm{C}$ isotherm, suggesting that these two events are coupled.

Elimination of carbodiimide would produce bis-N,N,-dimethyl-N',N"diisopropyl dimethylamido guanidinatoindium(III) (2, Fig. 2.2). This compound was synthesized as a yellow liquid in low yield by two successive salt metathesis reactions, beginning with indium trichloride: with the lithium guanidinate and two equivalents of amide, respectively. Compound $\mathbf{2}$ was found to have a poor TG trace, with several decomposition features (Fig. 2.7b).

The thermolysis of $\mathbf{2}$ was enlightening in understanding the thermal chemistry of $\mathbf{1}$. Although $\mathbf{1}$ appeared to have a suitable TG trace with a derivative curve representative of volatilization (Fig. 2.7a), the high residual mass suggested decomposition was occurring. The presence of carbodiimide in the previous thermo- 

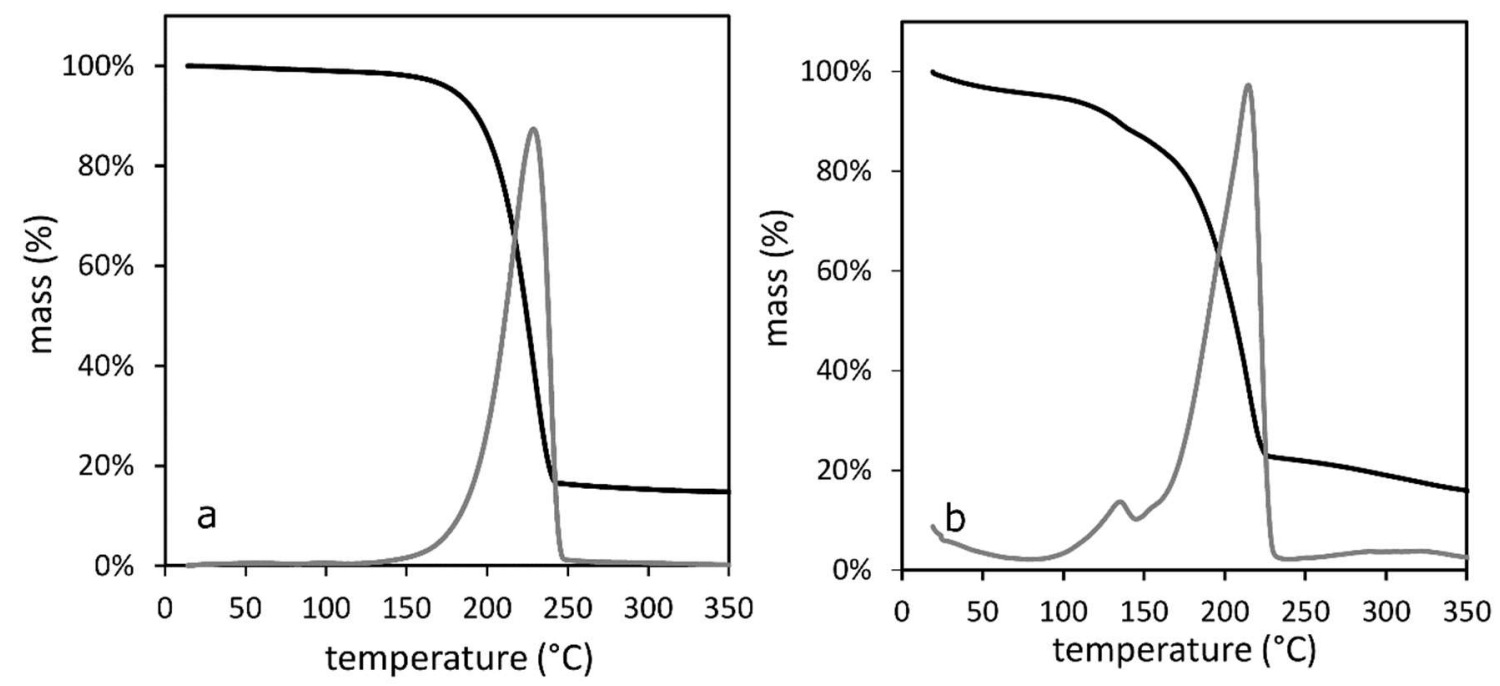

Figure 2.7: Standard ramp TGs of compounds 1 (a) and $\mathbf{2}$ (b). Derivative curves are in grey.

lyses ${ }^{1} \mathrm{H}$ NMR ${ }^{78,80}$ suggests that $\mathbf{1}$ is at least partially converting to $\mathbf{2}$, or another heteroleptic derivative of $\mathbf{1}$. The TG for $\mathbf{2}$ showed more resolved decomposition and volatilization steps, and had a significantly high residual mass (25\%) that itself continued to decompose as the temperature increased (Fig. 2.7b). The residual mass was a grey, charred solid. The ${ }^{1} \mathrm{H}$ NMR evidence from sealed, thermolyzed samples again showed CDI elimination, suggesting that the residual mass is somehow related to the monoguanidinato species 3 (Fig. 2.2).

Synthetic efforts to make and isolate $\mathbf{3}$ were surprisingly unsuccessful, with the reaction mass becoming a viscous, intractable paste. Although several methods of work-up were employed, no characterizable material could be isolated from the reaction mixture. We hypothesize that the low coordination number and the large indium centre together allowed the material to form a large order oligomer (or 
possibly a polymer), preventing isolation. However, this fits well with the thermal chemistry seen above. If $\mathbf{1}$ underwent successive CDI eliminations to ultimately form a non-volatile polymer of $\mathbf{3}$, this would provide the residual mass seen in both TG traces in Fig. 2.7.

The evolution of CDI from 1 was followed over two weeks in a sealed NMR tube. The tube was heated in steps to $150{ }^{\circ} \mathrm{C}(2$ days $), 160{ }^{\circ} \mathrm{C}(7$ days $), 170{ }^{\circ} \mathrm{C}(3$ days), and $180{ }^{\circ} \mathrm{C}$ (2 days) (Fig. 2.8).

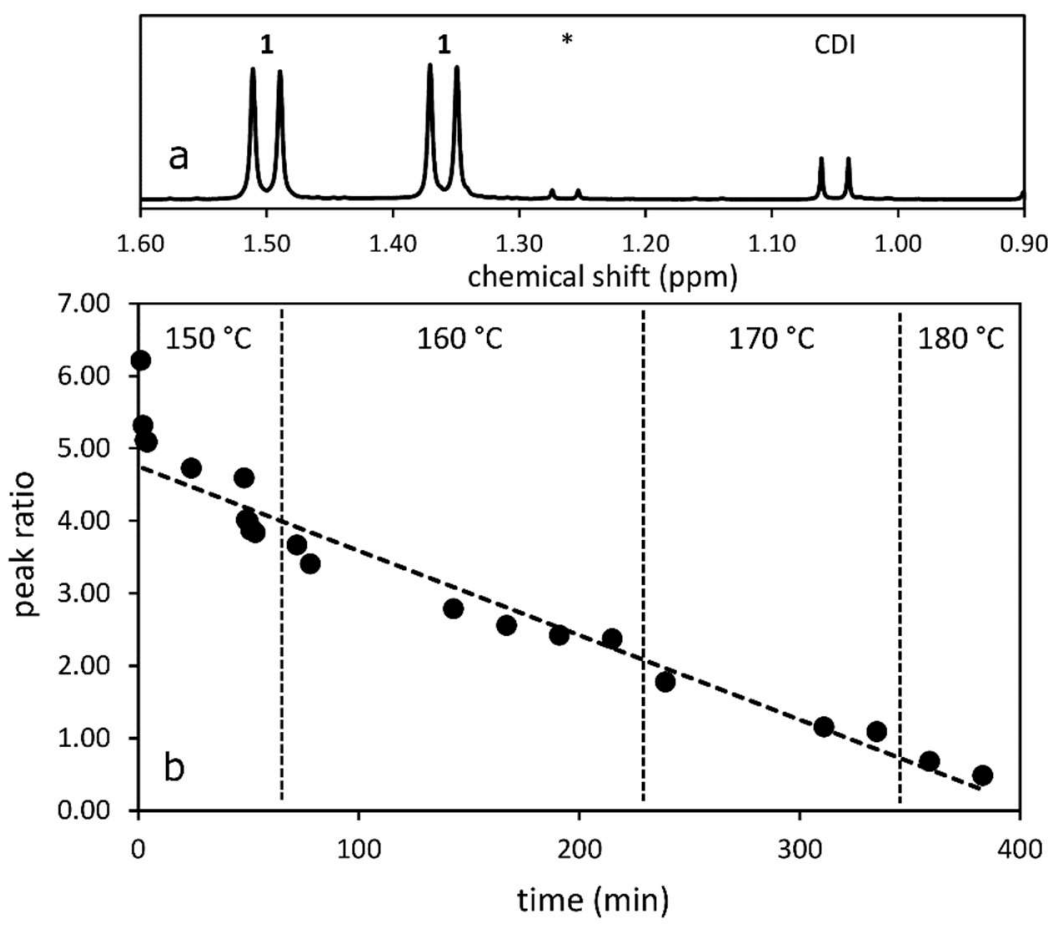

Figure 2.8: The ${ }^{1} \mathrm{H}$ NMR study of thermolysis of 1 . a) the NMR peaks used to determine thermolysis (* shows the minor guanidine thermolysis product). b) a graph of the ratio of the areas for $\mathbf{1}$ vs. CDI. The coefficient of determination $\left(\mathrm{R}^{2}\right)$ was 0.96 for the fitted line.

The peak ratio in the ${ }^{1} \mathrm{H}$ NMR for the doublets of $\mathbf{1}$ against the doublet for CDI (Fig. 2.8a) showed that the rate of decomposition was relatively constant over 
2 weeks, even as temperature was raised from $150{ }^{\circ} \mathrm{C}$ to $180{ }^{\circ} \mathrm{C}$. It should be noted that the first two data points (within 2 hours of sealing and heating the tube) were excluded. Commonly, even the most carefully sealed tube will show degradation of the product due to flame-sealing the NMR tube. The constant decline of $\mathbf{1}$ over this temperature range again demonstrated the thermal instability at temperatures near the necessary bubbler temperature of $\mathbf{1}$, and suggested further that CDI was a major by-product of decomposition. However, the ${ }^{1} \mathrm{H}$ NMR also showed characteristic peaks for protonated guanidine, as well as other septet and doublet environments that represent environments of $\mathbf{2}$ and potentially other isopropylcontaining ligand environments. This showed that CDI elimination was not the only thermal process that $\mathbf{1}$ undergoes. Additionally, the quantification of these data is provisory: there was significant precipitate that formed during the thermolysis. Again, we suspect this to be intractable 3, but this material was uncharacterized.

\subsection{Summary and Conclusions}

Compound 1 showed a low-lying and complex thermolysis that could affect its use in deposition conditions. The previously reported temperatures for evolving vapor

from 1 were $130{ }^{\circ} \mathrm{C}^{12}$ for $\mathrm{ALD}$ and $150{ }^{\circ} \mathrm{C}^{11}$ for $\mathrm{CVD}$. The thermolysis of $\mathbf{1}$ to the intermediate bisguanidinate $\mathbf{2}$ and ultimately to the non-volatile monoguanidinate 
3 through CDI elimination was calculated to establish an equilibrium around 120 ${ }^{\circ} \mathrm{C}$, with both CDI elimination steps being thermodynamically favorable above 230 ${ }^{\circ} \mathrm{C}$. The precursor can therefore be used as a deposition precursor, but its decomposition in the bubbler is expected to start immediately.

The decomposition studies by ${ }^{1} \mathrm{H}$ NMR suggest that the decomposition will occur over a period of weeks, and that it is relatively insensitive to temperature in the range of $150{ }^{\circ} \mathrm{C}$ to $180{ }^{\circ} \mathrm{C}$. The major product of decomposition is diisoproylcarbodiimide, and this elimination can lead having a vapor mixture containing both species $\mathbf{1}$ and $\mathbf{2}$, while also producing a nonvolatile decomposition byproduct 3. Some free guanidine was also found as a byproduct of the NMR thermolysis experiment, but this pathway seems to be a significant minority.

The precursor $\mathbf{1}$ has been successfully used in both CVD and ALD deposition conditions, but in both reported processes, there was noted difficulties with the precursor. After extensive thermal testing, it appears that the compound does not degrade significantly faster as temperature is increased, but has a steady thermal decomposition mainly due to carbodiimide elimination. This suggests that 1 can be successfully used for indium nitride deposition, but substantial amounts of precursor should not be stored in the bubbler for multiple runs. Rather, small amounts of precursor should be freshly isolated and used for each new experiment, to minimize thermal losses and to limit complicated surface chemistry by multiple 
gas phase species (e.g., 1, 2, carbodiimide, and guanidine). Deposition of indium nitride by plasma and thermal methods are presently being studied using this $\mathbf{1}$.

\subsection{Acknowledgements}

This project was founded by the Swedish foundation for Strategic Research through the project "Time-resolved low temperature CVD for III-nitrides" (SSF-RMA 150018). HP acknowledges the Wenner-Gren foundations and the COST Action MP1402 'Hooking together European research in atomic layer deposition (HERALD) supported by COST (European Cooperation in Science and Technology) for funding for a sabbatical at Carleton University. STB and SCB acknowledge the Vinnova VINNMER Marie Curie incoming mobility program for funding for a sabbatical and research visits to Linköping University (Vinnova grant 2015-03714). HP and STB are very grateful for the networking support provided from the COST Action HERALD. LO acknowledges financial support from the Swedish Government Strategic Research Area in Materials Science on Functional Materials at Linköping University (Faculty Grant SFO Mat LiU No. 2009 00971). LO acknowledges super- computing resources were provided by the Swedish National Infrastructure for Computing (SNIC) and the Swedish National Supercomputer Centre (NSC) and the Swedish Research Council (VR grant 201605137_4). 


\subsection{Supporting Information}
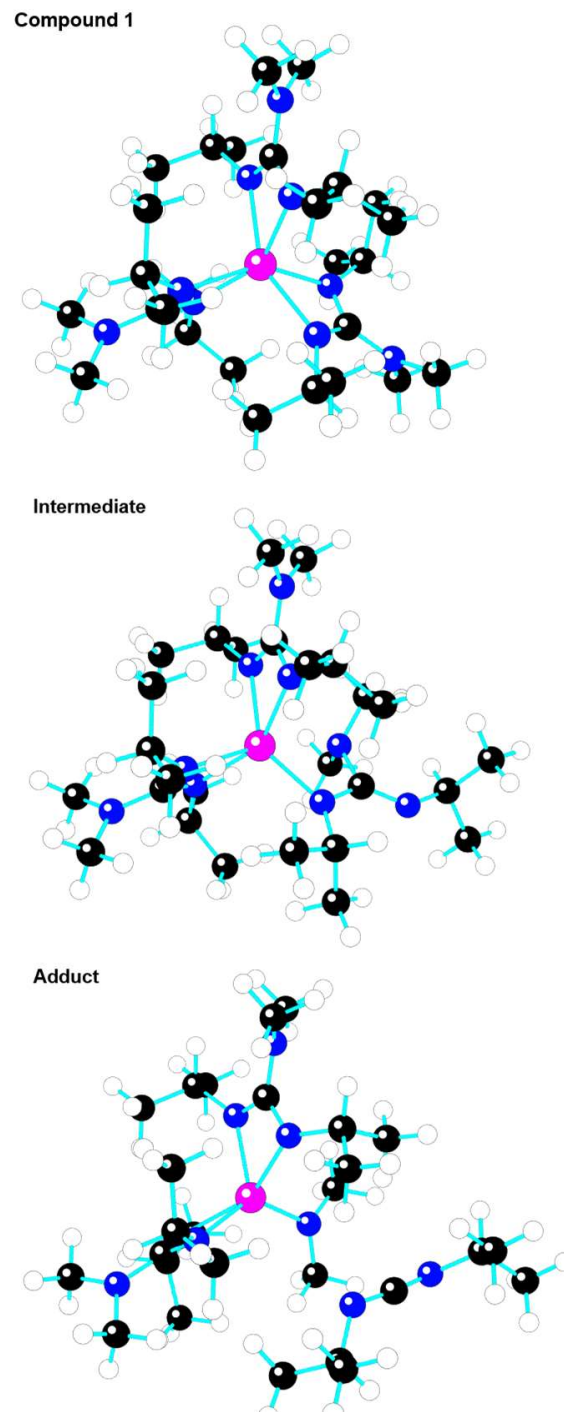

Compound 3
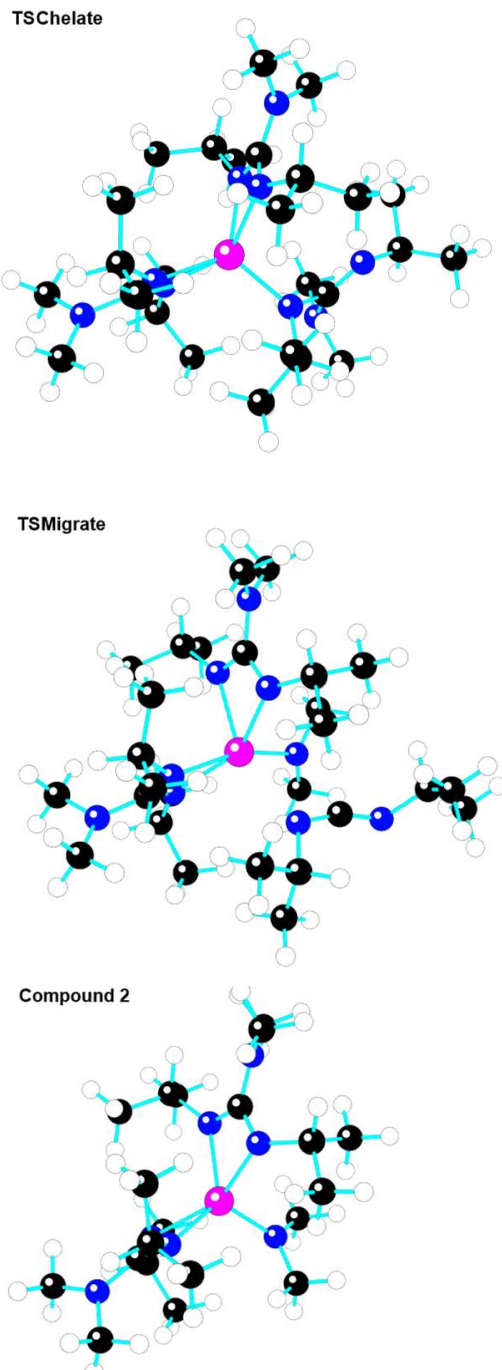

iPrCDI

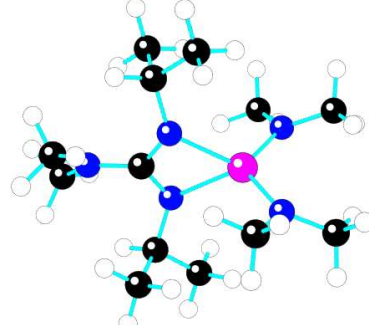

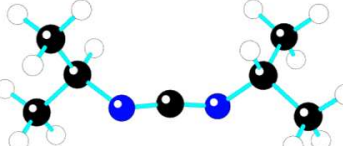

Figure 2.9: Converged structures of the different compounds using B3LYP/6$31 \mathrm{G}+(\mathrm{d}, \mathrm{p})$, lanl2dz. 
Table 2.2: Important bond lengths in the different structures given in $\AA$.

\begin{tabular}{lllllllll}
\hline & $\mathbf{1}$ & TS1 & Intermediate & TS2 & Adduct & $\mathbf{2}$ & $\mathbf{3}$ & iPrCDI \\
\hline In-N1 & 2.24 & 2.05 & 2.11 & 2.49 & 3.98 & 2.16 & 2.17 & $N / A$ \\
In-N2 & 2.24 & 3.58 & $N / A$ & $N / A$ & $N / A$ & 2.26 & 2.17 & $N / A$ \\
In-N3 & $N / A$ & 3.77 & 2.59 & 2.11 & 2.03 & $N / A$ & $N / A$ & $N / A$ \\
In-N4 & $N / A$ & $N / A$ & $N / A$ & $N / A$ & $N / A$ & 2.03 & 2.00 & $N / A$ \\
C-N1 & 1.34 & 1.40 & 1.36 & 1.26 & 1.23 & 1.35 & 1.35 & 1.23 \\
C-N2 & 1.34 & 1.28 & 1.28 & 1.21 & 1.22 & 1.34 & 1.35 & 1.23 \\
C-N3 & 1.40 & 1.45 & 1.48 & 2.48 & 3.45 & 1.39 & 1.38 & $N / A$ \\
\hline
\end{tabular}

Note: $N 1$ and N2 are the two formamidine nitrogen. $N 3$ is the dimethyl nitrogen. $N_{4}$ is the dimethyl nitrogen bonded direct to the indium in 2 and 3. $C$ is the central guanidinate carbon.

Table 2.3: Relative free energy for the compounds. All energies are given in $\mathrm{kJ} / \mathrm{mol}$ relative to 1 .

\begin{tabular}{|c|c|c|c|c|c|c|}
\hline $\mathrm{T}\left({ }^{\circ} \mathrm{C}\right)$ & TS1 & Intermediate & TS2 & Adduct & $\begin{array}{c}\text { Separated } \\
\mathbf{2}+\text { iPrCDI }\end{array}$ & $\begin{array}{c}\text { Separated } \\
\mathbf{3}+2 \text { iPrCDI }\end{array}$ \\
\hline 0 & 132.4 & 33.9 & 149.7 & 112.4 & 59.5 & 127.4 \\
\hline 10 & 132.3 & 33.7 & 149.4 & 111.7 & 56.7 & 122.0 \\
\hline 20 & 132.1 & 33.5 & 149.0 & 111.0 & 53.8 & 116.6 \\
\hline 30 & 131.9 & 33.3 & 148.7 & 110.3 & 51.0 & 111.1 \\
\hline 40 & 131.8 & 33.1 & 148.3 & 109.6 & 48.1 & 105.7 \\
\hline 50 & 131.6 & 32.9 & 147.9 & 108.8 & 45.3 & 100.3 \\
\hline 60 & 131.5 & 32.7 & 147.6 & 108.1 & 42.5 & 94.9 \\
\hline 70 & 131.4 & 32.5 & 147.2 & 107.4 & 39.6 & 89.5 \\
\hline 80 & 131.2 & 32.3 & 146.9 & 106.7 & 36.8 & 84.1 \\
\hline 90 & 131.1 & 32.1 & 146.5 & 106.0 & 34.0 & 78.6 \\
\hline 100 & 130.9 & 31.9 & 146.1 & 105.2 & 31.1 & 73.2 \\
\hline 110 & 130.8 & 31.7 & 145.8 & 104.5 & 28.3 & 67.8 \\
\hline 120 & 130.7 & 31.5 & 145.4 & 103.8 & 25.5 & 62.4 \\
\hline 130 & 130.5 & 31.3 & 145.0 & 103.1 & 22.6 & 57.0 \\
\hline 140 & 130.4 & 31.1 & 144.7 & 102.3 & 19.8 & 51.6 \\
\hline 150 & 130.3 & 30.9 & 144.3 & 101.6 & 17.0 & 46.2 \\
\hline 160 & 130.1 & 30.7 & 144.0 & 100.9 & 14.2 & 40.9 \\
\hline 170 & 130.0 & 30.5 & 143.6 & 100.1 & 11.3 & 35.5 \\
\hline 180 & 129.9 & 30.3 & 143.2 & 99.4 & 8.5 & 30.1 \\
\hline 190 & 129.8 & 30.1 & 142.9 & 98.7 & 5.7 & 24.7 \\
\hline 200 & 129.6 & 29.9 & 142.5 & 97.9 & 2.9 & 19.3 \\
\hline 210 & 129.5 & 29.7 & 142.1 & 97.2 & 0.1 & 14.0 \\
\hline 220 & 129.4 & 29.5 & 141.8 & 96.4 & -2.7 & 8.6 \\
\hline 230 & 129.3 & 29.3 & 141.4 & 95.7 & -5.6 & 3.2 \\
\hline 240 & 129.2 & 29.1 & 141.1 & 95.0 & -8.4 & -2.1 \\
\hline 250 & 129.1 & 28.9 & 140.7 & 94.2 & -11.2 & -7.5 \\
\hline 260 & 128.9 & 28.7 & 140.3 & 93.5 & -14.0 & -12.8 \\
\hline 270 & 128.8 & 28.4 & 140.0 & 92.7 & -16.8 & -18.2 \\
\hline 280 & 128.7 & 28.2 & 139.6 & 92.0 & -19.6 & -23.5 \\
\hline 290 & 128.6 & 28.0 & 139.3 & 91.2 & -22.4 & -28.9 \\
\hline 300 & 128.5 & 27.8 & 138.9 & 90.5 & -25.2 & -34.2 \\
\hline
\end{tabular}




\section{Chapter 3 - Part I}

\section{Tris(dimethylamido)aluminum(III):}

\section{An overlooked ALD precursor}

Modified from the original manuscript published as:

Buttera, S. C.; Mandia, D. J.; Barry, S. T. Tris(Dimethylamido)Aluminum(III):

An Overlooked Atomic Layer Deposition Precursor. J. Vac. Sci. Technol. A

Vacuum, Surfaces, Film. 2017, 35 (1), 01B128. https://doi.org/10.1116/1.4972469. 


\subsubsection{Abstract}

Aluminum oxide and aluminum nitride-containing films were grown by atomic layer deposition (ALD) and plasma-enhanced atomic layer deposition (PE-ALD) by employing an under-utilized tris(dimethylamido)aluminum(III) precursor. This compound has not been reported as a precursor for ALD of alumina previously, and has only been reported as an AlN precursor for a thermal process using ammonia as a co-reagent. Thermogravimetric analysis (TGA) demonstrates its excellent volatility and thermal stability, both of which are ideal characteristics for an ALD precursor. Aluminum oxide films were deposited thermally using water as a co-reagent. By x-ray photoelectron spectroscopy (XPS), the films appeared nearly pristine with only adventitious carbon on the surface accumulated post-deposition that was easily removed with 2 minutes of $\mathrm{Ar}^{+}$sputtering. The rest of the films contained a very low $1.4 \%$ impurity of carbon. Aluminum nitride films were attempted using the same aluminum precursor with nitrogen plasma as a coreagent; they contained large amounts of oxygen due to ambient exposure, possible oxidation during characterization, or the presence of incidental oxygen during the deposition of AlN, which allowed the formation of an aluminum oxynitride. Though the composition was not stoichiometrically AlN, the films also contained $\sim 1 \%$ carbon impurities, which is an improvement over many other AlN films reported, 
particularly those using TMA as a precursor. This precursor shows great promise for the deposition of low-impurity or impurity-free aluminum nitride by PE-ALD.

\subsubsection{Introduction}

Amidinates and similar ligands are now familiar in atomic layer deposition (ALD). ${ }^{55}$ These ligands have been used for the deposition of a wide range of materials with a variety of electronic properties. Our research continues an abiding interest in the modification of this ligand framework, specifically to make guanidinates ${ }^{86}$ and iminopyrrolidinates. ${ }^{87}$ The main reason for the synthesis of iminopyrrolidinates was to circumvent low-lying thermal decomposition pathways in both amidinates and guanidinates: these compounds were found to undergo betahydrogen abstraction ${ }^{64}$ and carbodiimide elimination ${ }^{81}$ at temperatures as low as $25^{\circ} \mathrm{C}$ (for the $\mathrm{N}, \mathrm{N}^{\prime}, \mathrm{N}^{\prime \prime}$-triisopropylguanidinatogold(I) dimer) ${ }^{88}$ (Figure 3.1).

Carbodiimide elimination is an interesting decomposition route for amidinates and guanidinates, since it is the reverse reaction of the simplest synthetic route to synthesize these compounds (i.e., carbodiimide insertion, Figure 3.1c). ${ }^{55}$ This reaction has been best exploited in cases where the amide of a given metal is not thermally stable or very volatile, but the amidinate or guanidinate is. Copper is an excellent example of this: the most thermally stable and volatile copper(I) amide is $\left[\mathrm{CuN}\left(\mathrm{SiMe}_{3}\right)\right]_{4}$, which has never been successfully employed for 
ALD. ${ }^{89}$ However, the amidinate is easy to make and modify, and is a famous ALD precursor for copper metal. ${ }^{90}$ We have undertaken research on the synthesis and volatility of aluminum guanidinates as ALD precursors, and identified some potentially interesting candidates. ${ }^{86}$ During this study, it became apparent that the starting material, tris(dimethylamido)aluminum(III) (TDMAA) has never been reported as an ALD precursor for alumina. It has been used in the deposition of aluminum nitride using ammonia as a co-reagent in a thermal process, but also has never been used to deposit AlN by PE-ALD. ${ }^{91}$ This is likely due to the fact that TMA is readily available, extremely well studied, and simple to use for the deposition of aluminum containing species.

In recent years, aluminum nitride has been of particular interest since it is a large band gap material that is desirable for its high resistivity and piezoelectric coupling coefficient. ${ }^{92}$ AlN can be deposited by several methods such as molecular beam epitaxy (MBE) and physical vapor deposition (PVD), ${ }^{93}$ but neither of these techniques allows for the precise thickness and conformality control of ALD. Though the deposition of aluminum oxide has been thoroughly studied in ALD research in the past, ${ }^{22}$ a great majority of this research pertains to the use of TMA as a precursor. As mentioned above, TMA is an extremely valuable precursor as it is readily available and consistently deposits aluminum oxide and nitride films. 
However, something can be said for the benefits of depositions using novel precursors. Film deposition using novel precursors is beneficial not only in the realm<smiles>[R]C1=NN([R1])C([R2])=[N+]([R])N=[N+]1[R]</smiles>

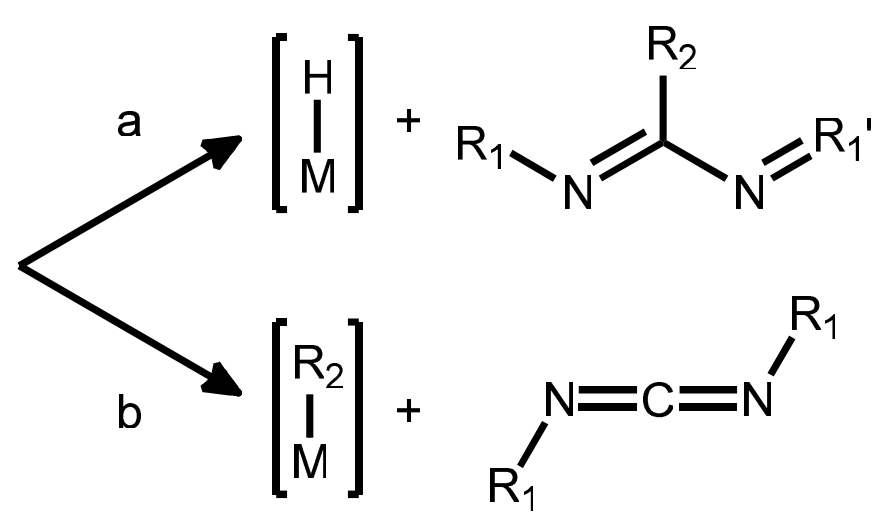
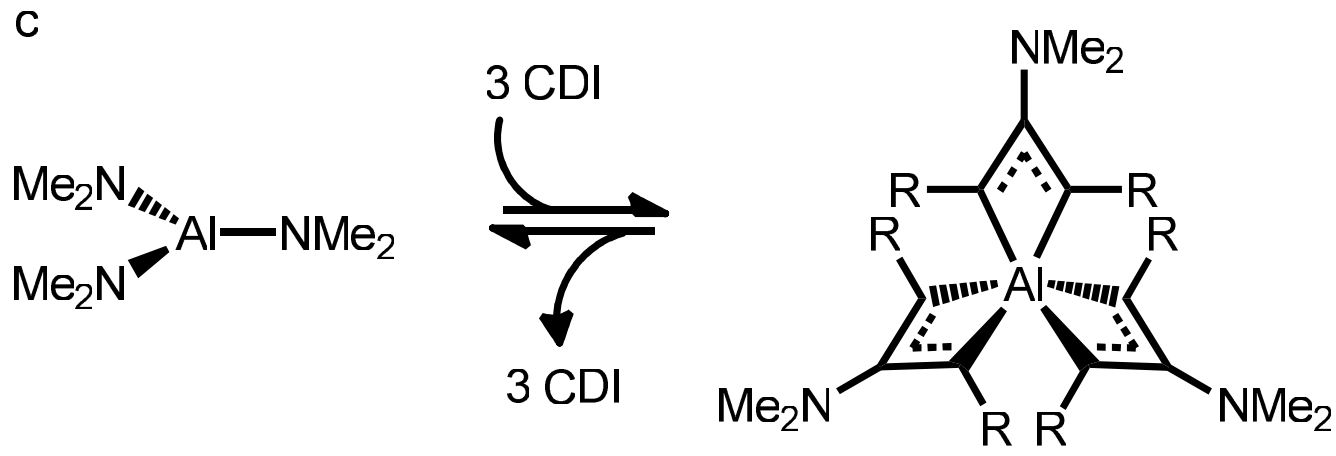

Figure 3.1: Common decomposition pathways for amidinate and guanidinate compounds; a) is beta-hydrogen abstraction from $R_{1}$ by the metal center and $b$ ) is carbodiimide elimination after $\mathrm{R}_{2}$ is abstracted by the metal center. Figure $3.1 \mathrm{c}$ ) shows the reversible insertion/elimination of carbodiimide (CDI) from trisdimethylamidoaluminum(III) (TDMAA).

of fundamental chemistry, but also for possible practical applications stemming from a difference in film quality and characteristics compared to those deposited by TMA. Considering the use of TDMAA specifically, the impurity level of carbon commonly observed (specifically in aluminum nitride films) could be reduced by employing a precursor in which aluminum has no direct metal-carbon bonds. 
Herein, we report an investigation of tris(dimethylamido)aluminum(III) (TDMAA)

as a precursor for the deposition of both aluminum oxide and aluminum nitride.

\subsubsection{Experimental}

\section{Precursor Synthesis}

All reactions were performed in an MBRAUN Labmaster 130 nitrogen dry box.

Lithium dimethylamide and aluminum trichloride were used as purchased from

Sigma Aldrich while solvents were purchased from VWR and purified in an MBRAUN Manual Solvent Purification.

In a $250 \mathrm{~mL}$ flask, lithium dimethylamide $(2.909 \mathrm{~g}, 57 \mathrm{mmol})$ was suspended in approximately $100 \mathrm{~mL}$ of dried diethyl ether and $50 \mathrm{~mL}$ of dried hexanes and was stirred vigorously. Aluminum trichloride (2.690 g, $20 \mathrm{mmol})$ was dissolved in approximately $30 \mathrm{~mL}$ of dried diethyl ether and both solutions were placed in a $30{ }^{\circ} \mathrm{C}$ freezer for 20 minutes. The lithium dimethylamide solution was then added dropwise by pipette to the aluminum chloride solution with stirring. The mixture was allowed to warm to room temperature and stirred for 18 hours. The solid $\mathrm{LiCl}$ precipitate was removed via vacuum filtration, and the remaining solution was then dried down by vacuum and taken back up in approximately $50 \mathrm{~mL}$ of dried hexanes and filtered again. This filtered solution was dried down by vacuum to a minimum volume and allowed to recrystallize in the freezer. Colourless crystals were 
obtained, which matched the literature reported characterization of TDMAA. ${ }^{94}$

\section{Thermogravimetric Analysis}

A TA Instruments TGA Q500 tool was used for all thermogravimetric analyses. For the ramp experiment of TDMAA, $44 \mathrm{mg}$ of precursor was added to a tared pan and loaded into the furnace. The furnace was heated at a rate of $10{ }^{\circ} \mathrm{C} /$ minute up to $600{ }^{\circ} \mathrm{C}$ while nitrogen flow rates were set to $60 \mathrm{sccm}$. No significant decomposition of the precursor occurred $(<2 \%$ residual mass). In the stepped isothermal experiment, ${ }^{10}$ a sample mass of $45 \mathrm{mg}$ was subjected to several isothermal events (from 60 to $120{ }^{\circ} \mathrm{C}$ ) for seven minute periods, followed by ramps of $40{ }^{\circ} \mathrm{C} /$ minute to reach the proceeding step. TDMAA showed linear mass loss over time and a negligible residual mass of less than $2 \%$. The compound exhibited 1 Torr of vapor pressure at $78{ }^{\circ} \mathrm{C}$, measured using mass loss data and an instrumental calorimetric constant with the Knudsen equation.

\section{Thin Film Deposition}

All aluminum oxide and aluminum nitride samples were grown on $\mathrm{Si}(100)$ coupons cleaned by the Piranha process. For experimental conditions on the depositions of films, see Tables 3.1 and 3.2 below. 
Table 3.1: Experimental conditions for the deposition of aluminum oxide thin films by ALD on a Picosun ALD R150 thermal tool. All experiments consisted of 500 cycle depositions and all pulses were followed by a 10 second nitrogen purge. Thicknesses were measured by ellipsometry.

\begin{tabular}{ccccc}
\hline TDMAA pulse (s) & $\begin{array}{c}\text { Bubbler } \\
\text { temperature }\left({ }^{\circ} \mathrm{C}\right)\end{array}$ & $\mathrm{H}_{2} \mathrm{O}$ pulse $(\mathrm{s})$ & $\begin{array}{c}\text { Reactor } \\
\text { temperature }\left({ }^{\circ} \mathrm{C}\right)\end{array}$ & $\begin{array}{c}\text { Average } \\
\text { thickness }(\AA)\end{array}$ \\
\hline 1 & 90 & 0.1 & 250 & 245 \\
3 & 90 & 0.1 & 250 & 443 \\
4 & 90 & 0.1 & 250 & 550 \\
6 & 90 & 0.1 & 250 & 534 \\
10 & 90 & 0.1 & 250 & 546 \\
\hline
\end{tabular}

Table 3.2: Experimental conditions for the deposition of aluminum nitride thin films by ALD on a Picosun R200 plasma tool. All experiments consisted of 500 cycle depositions and all pulses were followed by a 10 second nitrogen purge. Thicknesses were measured by ellipsometry.

\begin{tabular}{ccccc}
\hline TDMAA pulse (s) & $\begin{array}{c}\text { Bubbler } \\
\text { temperature }\left({ }^{\circ} \mathrm{C}\right)\end{array}$ & $\begin{array}{c}\mathrm{N}_{2} \text { plasma } \\
\text { pulse }(\mathrm{s})\end{array}$ & $\begin{array}{c}\text { Reactor } \\
\text { temperature }\left({ }^{\circ} \mathrm{C}\right)\end{array}$ & $\begin{array}{c}\text { Average } \\
\text { thickness }(\AA)\end{array}$ \\
\hline 2 & 90 & 8 & 250 & 244 \\
4 & 90 & 4 & 250 & 337 \\
4 & 90 & 8 & 250 & 382 \\
4 & 90 & 12 & 250 & 367 \\
8 & 90 & 8 & 250 & 392 \\
\hline
\end{tabular}

\section{Aluminum Oxide}

Deposition of aluminum oxide was performed on a Picosun ALD R150 thermal tool. A "PicoSolid" bubbler was employed to deliver TDMAA; the bubbler was filled in an MBRAUN Labmaster 130 nitrogen-filled drybox with between $500 \mathrm{mg}$ 
and $2 \mathrm{~g}$ of TDMAA. Milli-Q de-ionized water was used as the co-reagent for the deposition of these aluminum oxide thin films. A standard ALD cycle was performed as the follows: $4 \mathrm{~s}$ TDMAA pulse $\mid 10 \mathrm{~s} \mathrm{~N}_{2}(\geq 99.999 \%)$ purge $\mid 0.1 \mathrm{~s} \mathrm{H}_{2} \mathrm{O}$ pulse $10 \mathrm{~s} \mathrm{~N} \mathrm{~N}_{2}$ purge. Flows for precursors and purges were 100 and $80 \mathrm{sccm}$, respectively. The bubbler temperature for TDMAA was $90{ }^{\circ} \mathrm{C}$ in order to provide a sufficient vapor pressure while the reactor temperature was set to $250{ }^{\circ} \mathrm{C}$ for film growth.

\section{Aluminum Nitride}

Attempted deposition of aluminum nitride was performed on a Picosun R200 ALD plasma tool. For obtaining a saturating dose of both precursors to produce an aluminum nitride film, the experiment conditions were set as follows. The bubbler temperature for TDMAA was again $90{ }^{\circ} \mathrm{C}$ in order to provide a sufficient precursor vapor pressure (as above) while the reactor temperature was set to $250{ }^{\circ} \mathrm{C}$ for film growth. The bubbler containing TDMAA was pulsed with nitrogen carrier gas $(\geq 99.999 \%)$ at an extremely high flow $(1800 \mathrm{sccm})$ for 5 seconds prior to standard pulsing in order to increase subsequent mass delivery to the reactor chamber. TDMAA (with an overpressure of $\mathrm{N}_{2}$ ) was then pulsed for 4 seconds at a flow of $80 \mathrm{sccm}$, followed by a 10 second purge of nitrogen. Nitrogen plasma was ignited in a flow of argon at $2400 \mathrm{~W}$ power and was subsequently pulsed for 8 seconds. The cycle terminated with another $10 \mathrm{~s}$ nitrogen purge. 


\section{X-ray Photoelectron Spectroscopy}

XPS analysis was performed in a Specs (Gmbh) custom-built, multi-technique ultra-high vacuum system. The samples slides were cut down to $1 \mathrm{~cm}^{2}$, mounted on a copper sample holder and then evacuated for 2-3 hours in a load-lock chamber prior to sample transfer to the main manipulator arm in the XPS analysis chamber. Spectra were collected at background pressures of $3 \times 10^{-9}$ Torr using a $14.26 \mathrm{keV}$ $\mathrm{Al} \mathrm{K} \alpha$ source with a fixed sample angle of $\sim 1^{\circ}$. Spectra were collected in SpecsLab 2 software, post-processed in CasaXPS and rendered in Excel. $\mathrm{Ar}^{+}$sputtering cycles were performed at an acceleration voltage of $\sim 2 \mathrm{keV}$ under background Ar pressures in the range of $5 \times 10^{-6}-1 \times 10^{-5}$ Torr. Due to surface charging of the sample during spectral acquisition, the survey and high-resolution spectra were referenced to the adventitious carbon $\mathrm{C}$ 1s $(\mathrm{C}-\mathrm{C})$ signal at $284.8 \mathrm{eV}$. Peak deconvolution performed during post-processing was done using mixed Gaussian-Lorentzian functions. Relative sensitivity factors used for quantification and peak fitting of $\mathrm{Al}$ 2p, O 1s, C 1s, and N 1s from high-resolution scans were 0.537, 2.930, 1.000, and 1.800, respectively. Additionally, the high-resolution scans usually required increases to the hemispherical analyzer pass energy and dwell time of $\sim 10 \mathrm{eV}$ and $0.2 \mathrm{~s}$, respectively. 


\subsubsection{Results and Discussion}

TDMAA was made by straightforward salt metathesis of $\mathrm{AlCl}_{3}$ with three equivalents $\mathrm{LiNMe}_{2}$; the NMR was simple as expected. ${ }^{94}$ The thermogravimetric analysis of TDMAA showed clean volatilization with an onset of $58{ }^{\circ} \mathrm{C}$ (Figure 3.2a). It is instructive here to acknowledge the shape of the derivative curve: the exponential onset is characteristic of one kinetic step, and the rapid decline occurs due to lack of reactant left in the pan. This shape, accompanied by an approximately zero residual mass, is indicative of sublimation. To further characterize the vapor pressure, a stepped isotherm was measured (Figure 3.2b, after Umarji et. al.). ${ }^{20}$ Here, the vapour pressure was measured to be 1 Torr at 78 ${ }^{\circ} \mathrm{C}$, indicating that this amide is a very good precursor from a volatility and thermal stability point of view.

Deposition of aluminum oxide was carried out using TDMAA with a $90{ }^{\circ} \mathrm{C}$ bubbler temperature and $250{ }^{\circ} \mathrm{C}$ deposition temperature (Figure 3.3). The water pulses were kept at $0.1 \mathrm{~s}$ since water appeared to saturate at all pulse lengths. TDMAA showed saturative growth after $4 \mathrm{~s}$ pulses with a growth rate of $1.1 \AA \pm$ $0.1 \AA$, which is similar to the commonly expected growth rate of alumina deposited by TMA at this temperature. This is an interesting result since TDMAA appears to be notably bulkier than TMA. Using a simple approximation of molecular volume assuming spherical particles and estimating the particle volume from the 
mass density and the molecular weight, TMA has a molecular volume of $0.16 \mathrm{~nm}^{3}$ and TDMAA has a volume of $0.22 \mathrm{~nm}^{3}$, giving TDMAA a $12 \%$ larger diameter than TMA. The results and this simple calculation suggest that this difference in surface steric effect of each of these chemisorbed precursors at the surface did not affect the growth rate of alumina.
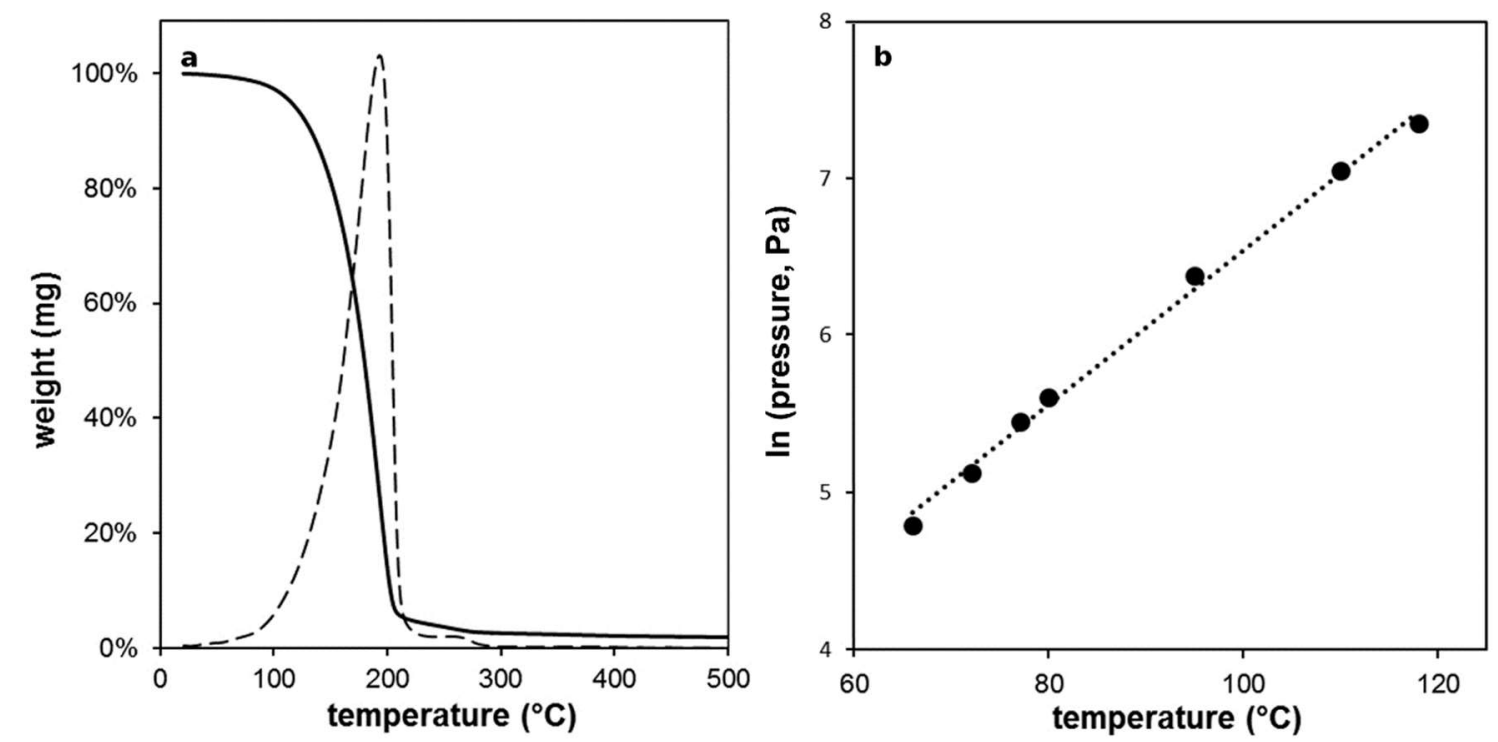

Figure 3.2: Thermogravimetric data for TDMAA. a) shows a ramped experiment at $10{ }^{\circ} \mathrm{C} / \mathrm{min}$ (solid line) as well as the derivative (dashed line). b) shows the vapor pressure at various temperatures derived from a stepped isothermal experiment, using the method described in reference 10. The fit (dotted line) has a coefficient of determination of 0.995. Pressure is given in Pascals.

The alumina samples grown on $\mathrm{Si}(100)$ were light brown in colour, visible by eye. All saturated films (i.e., at least a 4 s pulse of TDMAA) were even and consistent across coupons while those with under-saturating pulses (e.g., 2 s) showed a gradient on the substrate coupon from a brown film gradient to bare Si. Film composition was confirmed by energy-dispersive X-ray spectroscopy (EDX), 
although quantitative elemental composition could not be obtained due to the thinness of the films; the x-ray penetration depth greatly surpassed film thickness and traversed into the silicon substrate. All film thicknesses were measured using a Nanospec AFT film thickness measurement system assuming a refractive index of $1.65 .{ }^{95}$ Film composition was also studied by XPS (Figure 3.4 ) and showed nearly pure $\mathrm{Al}_{2} \mathrm{O}_{3}$ films with only adventitious carbon (likely from air-break) present on the surface $(284.5-284.8 \mathrm{eV})$; this was removed by sputtering for 2 minutes with a $2 \mathrm{KeV} \mathrm{Ar}^{+}$beam. This is comparable to or lower than other depositions of alumina using TMA as a precursor; it is a particularly lower contamination of carbon than is seen for TMA-deposited alumina at higher temperatures, demonstrating a potential advantage of using TDMAA over TMA for low-carbon film deposition. ${ }^{96}$

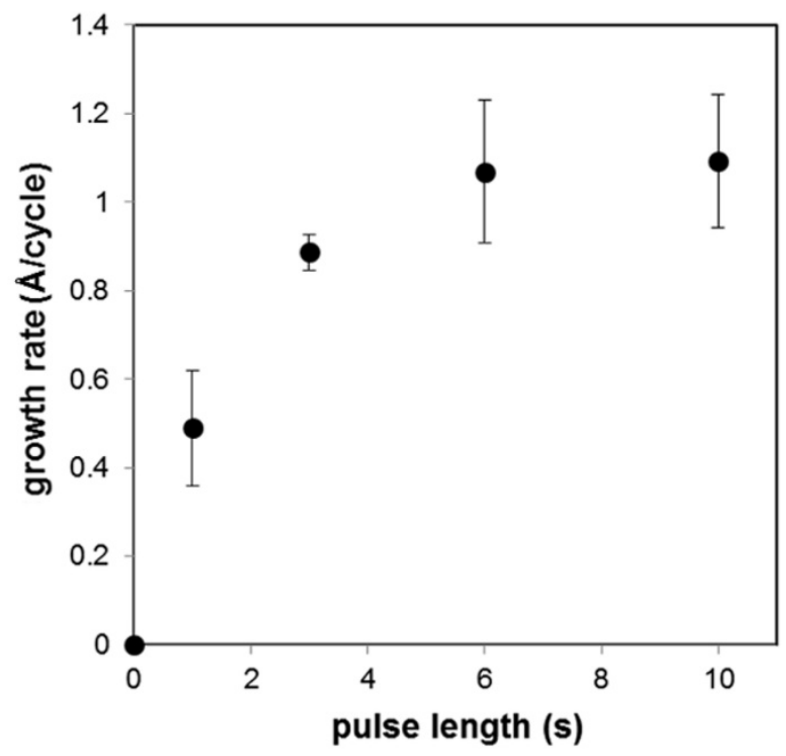

Figure 3.3: Saturation curve for TDMAA producing aluminum oxide with a $0.1 \mathrm{~s}$ water pulse in a thermal process. 


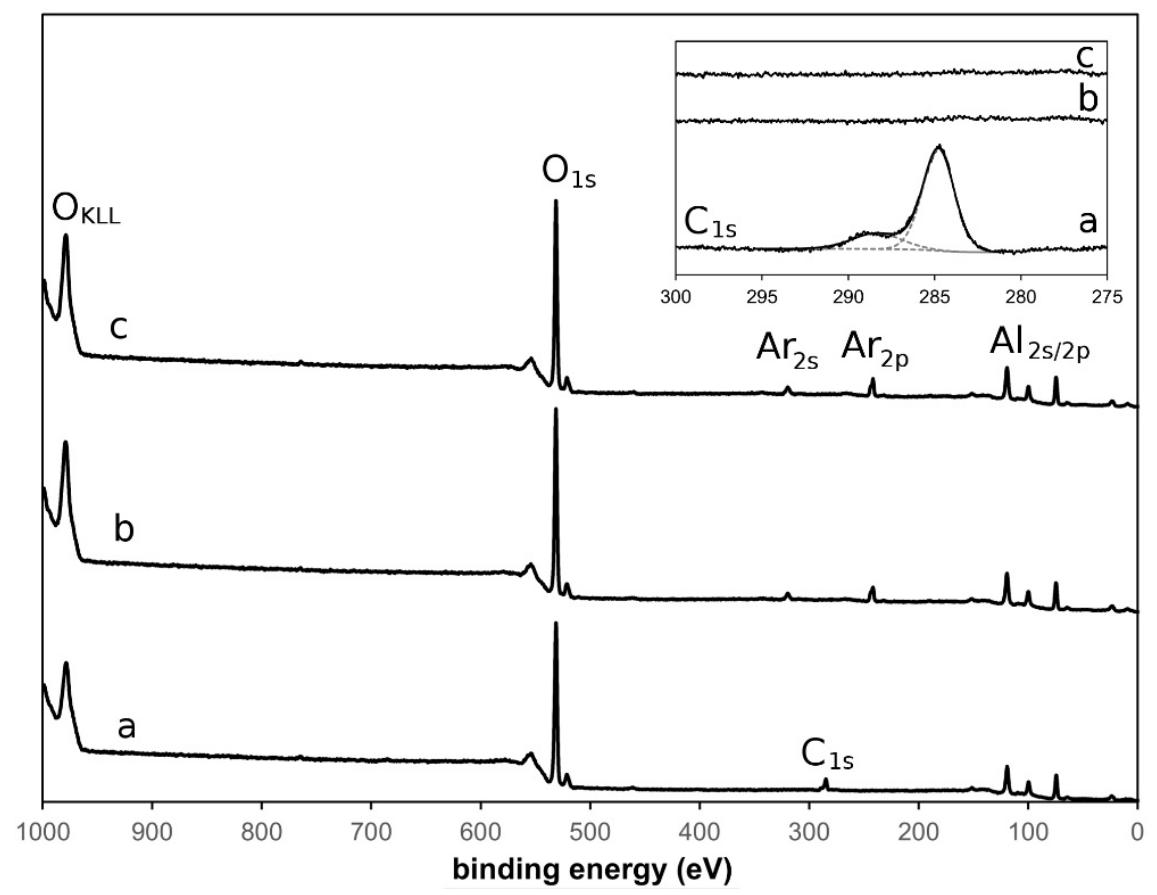

Figure 3.4: Survey XPS scan of aluminum oxide films deposited using a 6 s pulse of TDMAA where a) shows the film as deposited, b) after 2 minutes of $\mathrm{Ar}^{+}$ sputtering, and c) after 10 minutes of $\mathrm{Ar}^{+}$sputtering. Inset is a high-resolution scan of the $\mathrm{C} 1 \mathrm{~s}$ region, showing adventitious carbon on the surface, but not present throughout the film otherwise.

After using a $2 \mathrm{KeV} \mathrm{Ar}^{+}$beam to remove the surface contaminants, the $\mathrm{Al}: \mathrm{O}$ ratio increased slightly from 0.84 to 0.87 , and this then remained steady after even 10 minutes of sputtering. Although the $\mathrm{Al}: \mathrm{O}$ ratio was relatively stable, it deviated from the ideal (0.67). Potential preferential sputtering of oxygen and argon incorporation in the film complicated the analysis of the film's stoichiometry.

Attempted deposition of aluminum nitride was carried out using TDMAA with a bubbler temperature of $90{ }^{\circ} \mathrm{C}$ and nitrogen plasma, with the substrate again kept at $250{ }^{\circ} \mathrm{C}$ (Figure 3.5). The plasma power was $2400 \mathrm{~W}$ and its pulse time was $8 \mathrm{~s}$. Here, saturative growth was found after a $4 \mathrm{~s}$ pulse of TDMAA with a growth 
rate of $0.8 \AA \pm 0.06 \AA$ (Figure $3.5 \mathrm{a}$ ), which was lower than growth rates previously reported for TMA with a $\mathrm{N}_{2} / \mathrm{H}_{2}$ plasma $(1.2 \AA) .{ }^{49}$ Considering that the aluminum oxide from TDMAA showed almost the same growth rate as aluminum oxide from TMA under similar conditions, there are two likely reasons that this drop in growth rate could occur. If TMA chemisorbed easier to an aluminum nitride surface, then the drop in growth rate for TDMAA could be attributed to a less dense monolayer formed when using this precursor. Alternatively, it is likely that the lack of a reducing environment in the plasma (i.e., no dihydrogen to produce a hydrogen radical) allowed etching in this case compared to the TMA case. Further investigation of this growth rate by modeling and with a variety of different plasma precursors is warranted.
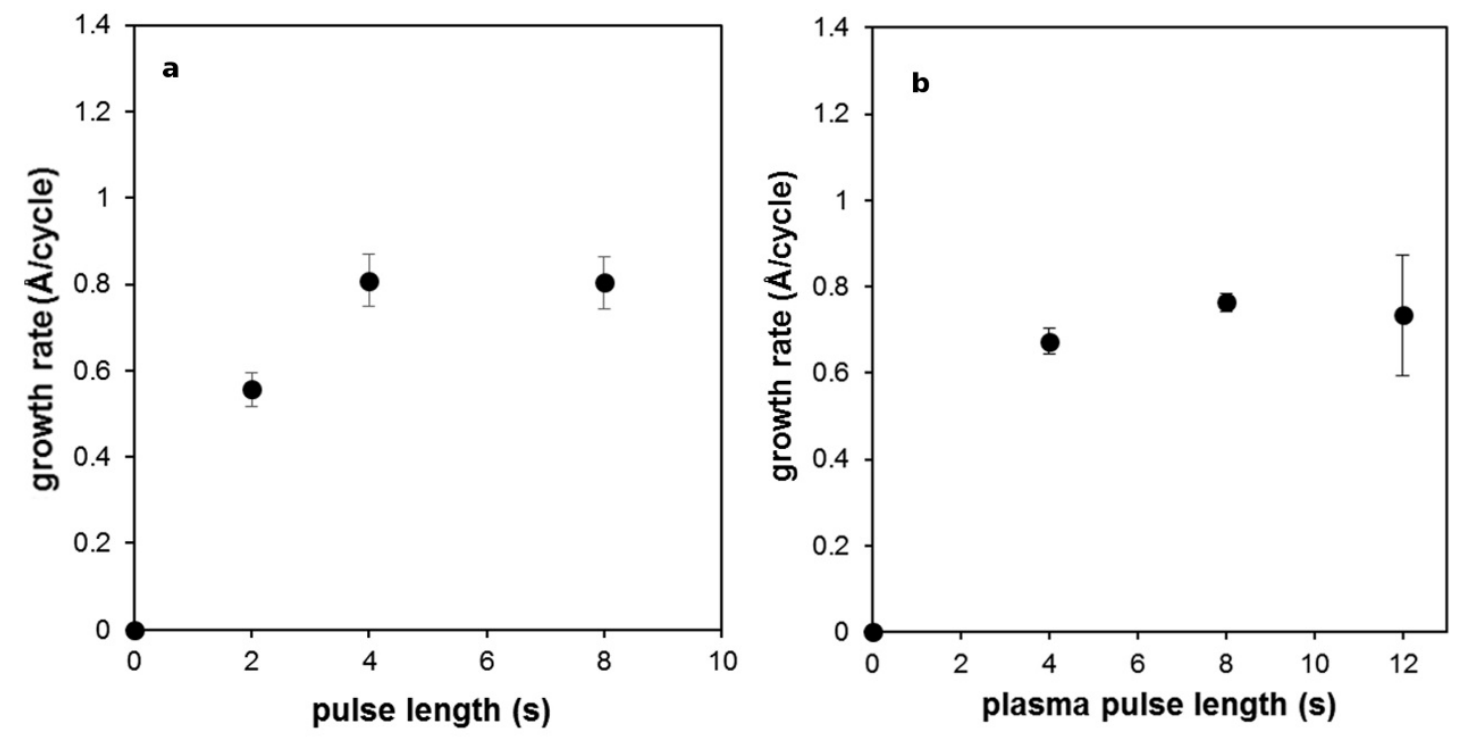

Figure 3.5: Saturation curves for TDMAA attempting aluminum nitride with nitrogen plasma while a) varying precursor pulse times keeping the plasma pulse at $8 \mathrm{~s}$, and b) varying plasma pulse times while keeping the pulse of TDMAA at 4 s. 
In addition to varying precursor pulse time to form a saturation curve, the plasma pulse time was also varied during a saturating dose of TDMAA of $4 \mathrm{~s}$. From these depositions (Figure 3.5b), it appears that a plasma time of $8 \mathrm{~s}$ is optimal for the deposition of this film. The plasma pulse of $4 \mathrm{~s}$ is insufficient, resulting in a lower growth rate possibly due to the short time allowed to react with the surface, and a pulse time of $12 \mathrm{~s}$ also results in a lower growth rate, possibly due to etching of the deposited films by an overexposure of plasma.

These films on $\mathrm{Si}(100)$ appeared beige to the eye. By scanning electron microscopy (SEM), the films appeared featureless and smooth. Similar to the alumina films, saturating doses of both precursors produced evenly coated substrates while non-saturating pulses of TDMAA or plasma produced a visible gradient. EDS clearly showed aluminum in the films, while the nitrogen signal was more difficult to isolate and again, was not quantifiable due to x-ray penetration into the silicon substrate. XPS survey scans of the film deposited with a $4 \mathrm{~s}$ precursor pulse were taken as-deposited, after a 2 minute $\mathrm{Ar}^{+}$sputter, and after a 10 minute $\mathrm{Ar}^{+}$sputter. All three scans showed major contributions from Al, N, and $\mathrm{O}$, suggesting that the film is more likely an aluminum oxynitride (Figure 3.6).

The as-deposited scan showed significant carbon and oxygen impurities, which can be attributed largely to adventitious species from the sample transfer. The car- 


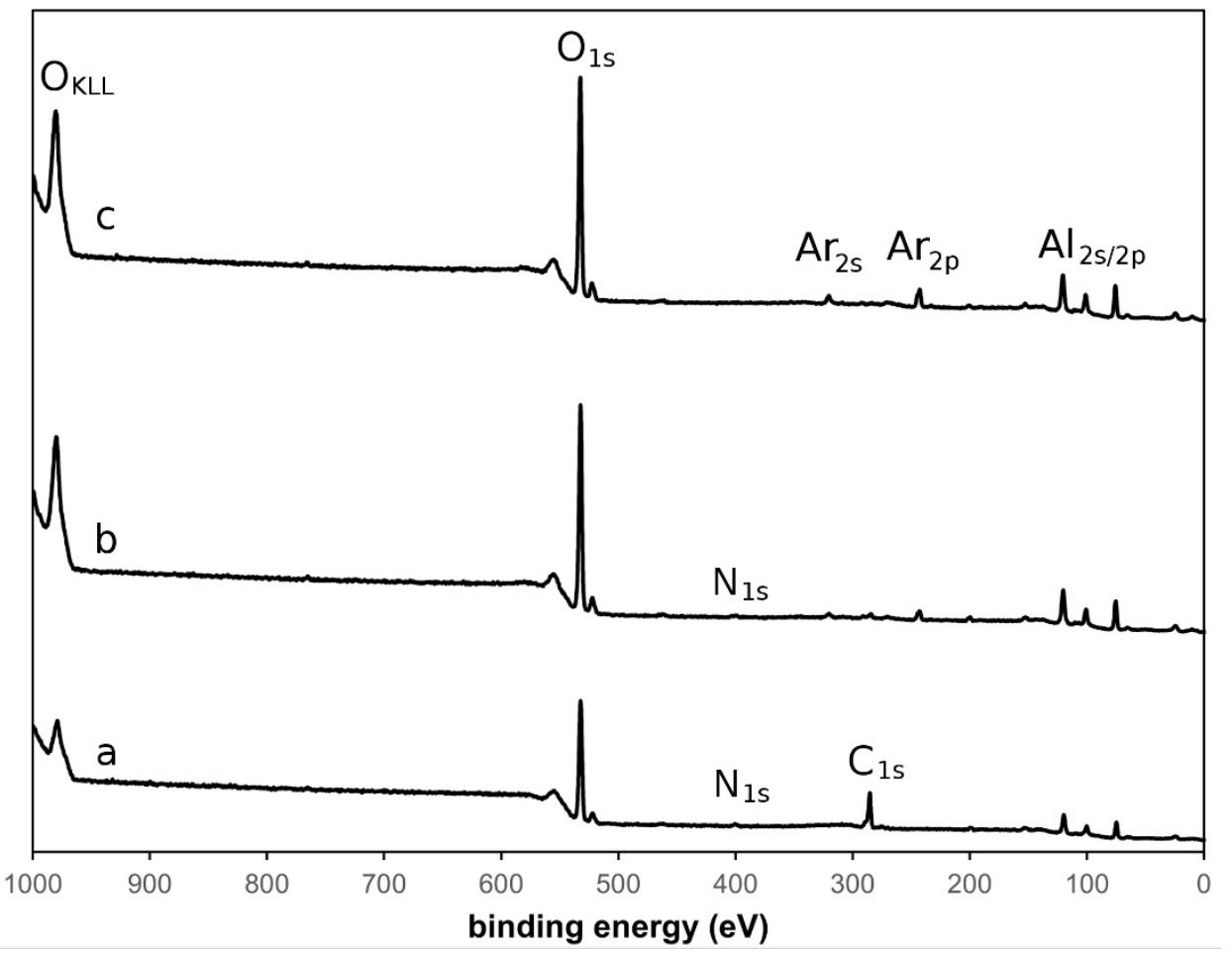

Figure 3.6: Survey XPS scans of films deposited using a 4 s pulse of TDMAA and an $8 \mathrm{~s}$ pulse of nitrogen plasma where a) shows the film as deposited, b) after 2 minutes of $\mathrm{Ar}^{+}$sputtering, and c) after 10 minutes of $\mathrm{Ar}^{+}$sputtering.

-bon peak was large at the surface, but dropped significantly to $\sim 1 \%$ after 2 minutes of sputtering with a $2 \mathrm{KeV} \mathrm{Ar}{ }^{+}$beam, where it remained even after 10 minutes of sputtering. Literature examples of aluminum nitride deposition using TMA have reported higher levels of carbon impurity in the ranges of $3-6 \%,{ }^{37,97-100}$ while aluminum nitride deposited from $\mathrm{AlCl}_{3}$ has shown similar levels of carbon to the present work. ${ }^{35,36}$ To that note, however, using $\mathrm{AlCl}_{3}$ as a precursor for aluminum nitride films can result in significant chloride incorporation $(<1$ to $3 \%)$. This again demonstrates the utility of TDMAA to lower carbon levels in deposited films compared to TMA. The elimination of carbon impurities from aluminum nitride 
films is important for the use of metal nitrides in electronics, as the presence of carbon, in even small quantities, will implicate an impurity band in the film's electronic structure. ${ }^{101}$ The observation of a notable oxygen peak in the asdeposited and sputtered samples indicated the presence of oxygen throughout the film. Surface oxygen was expected due to storage of the deposited films under atmosphere post-deposition and prior to characterization, but the bulk oxygen could originate from several sources. ${ }^{53}$ Because we have no method to transfer deposited films under an inert atmosphere or in vacuum, it was unclear whether the film reacted with oxygen during the transfer and storage or whether there was a significant oxygen source present in the plasma or purge gases of the reactor. However, XPS analysis showed that the Al:N ratio at the surface was 17:1, much higher than the expected stoichiometry. After sputtering for 2 minutes with a 2 $\mathrm{KeV} \mathrm{Ar}^{+}$beam, the Al:N ratio increased to 24:1. This could be due to preferential sputtering of nitrogen by the $\mathrm{Ar}^{+}$beam, but is also likely due to the high incorporation of oxygen (the Al:O ratio was 0.8). High resolution scans of the aluminum and nitrogen regions helped identify the role of oxygen in these films (Figure 3.7).

A change in the $\mathrm{N}$ 1s subshell occurred after two minutes of sputtering with a $2 \mathrm{KeV} \mathrm{Ar}{ }^{+}$beam: a new peak was found at $401.77 \mathrm{eV}$ next to the AlN peak centered at $397.83 \mathrm{eV}$. This peak can be attributed to an $\mathrm{N}^{+}$environment such as a nitrate 

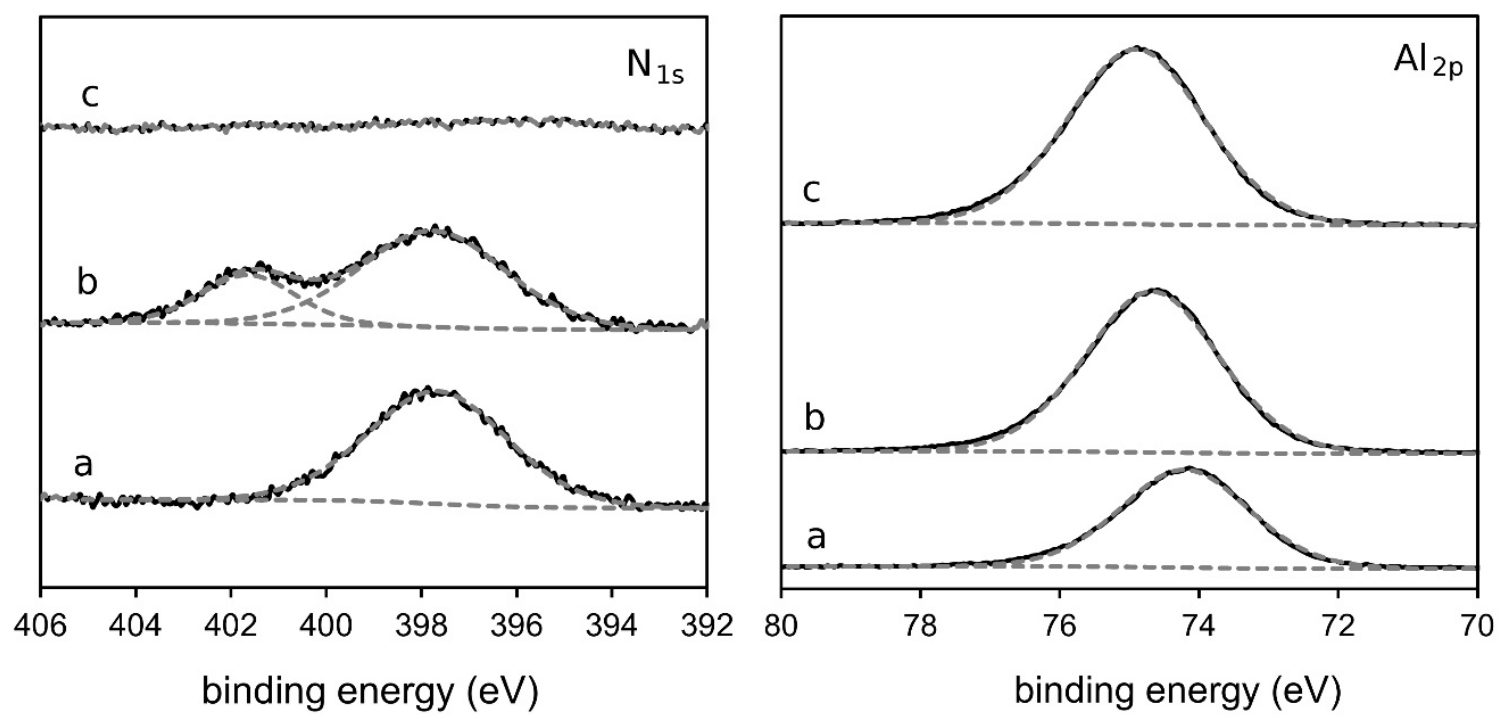

Figure 3.7: High resolution XPS scans of the $\mathrm{N} 1 \mathrm{~s}$ and $\mathrm{Al} 2 \mathrm{p}$ peak regions for films deposited using a $4 \mathrm{~s}$ TDMAA pulse and an $8 \mathrm{~s}$ nitrogen plasma pulse. a) shows the as deposited film, b) after 2 minutes of $\mathrm{Ar}^{+}$sputtering, and c) after 10 minutes of $\mathrm{Ar}^{+}$sputtering.

species, reactive $\mathrm{NO}$, or $\mathrm{NO}_{\mathrm{x}}$. This could be an indication of an aluminum oxynitride, ${ }^{102}$ forming O-Al-N environments or surface Al- $\mathrm{NO}_{\mathrm{x}}$ species. It is possible that preferential etching of $\mathrm{Al}$ caused by the $\mathrm{Ar}^{+}$-sputtering ${ }^{102}$ partially oxidized the remaining nitrogen, causing formation of these relatively stable $\mathrm{Al}-\mathrm{NO}_{\mathrm{x}}$ (or OAl-N-O) environments. The associated shift in the Al $2 p$ peak envelope from 74.11 $\mathrm{eV}$ (as-deposited) to $74.69 \mathrm{eV}$ after 2 minute $\mathrm{Ar}^{+}$-sputter and then finally to 75.54 eV after 10 minute $\mathrm{Ar}^{+}$-sputter showed the loss of nearly all $\mathrm{Al}-\mathrm{N}$ contributions and carbon impurities, resulting in almost pure $\mathrm{Al}_{2} \mathrm{O}_{3} .{ }^{103}$ This peak shift has previously been associated with the $\mathrm{Al} 2 \mathrm{p}$ binding energy for $\mathrm{Al}_{2} \mathrm{O}_{3}$ deposited by 
ALD. ${ }^{102,104}$ The Al:N ratio after 10 minutes of sputtering was 91:1, again indicating almost no AlN deep in the film.

The XPS analysis demonstrated that the as-deposited film was mostly composed of $\mathrm{Al}_{2} \mathrm{O}_{3}$ with a smaller component of AlN. This result suggests that there is a viable process for AlN deposition using TDMAA and nitrogen plasma, but great care of potential oxygen impurities needs to be undertaken. An extended study of refining this process by carefully controlling oxygen exposure and impurity is ongoing in our group.

\subsubsection{Summary and Conclusions}

The simple tris(dimethylamido)aluminum(III) precursor (TDMAA) was synthesized by salt metathesis and used as an ALD precursor for aluminum oxide and nitride films. Aluminum oxide films were grown thermally at $250{ }^{\circ} \mathrm{C}$ with water as a co-reagent and a saturating pulse for TDMAA of $4 \mathrm{~s}$. Films appeared light brown to the eye, corroborating with expected coloring for the thicknesses obtained and appeared featureless by SEM. Analysis by XPS showed nearly pure $\mathrm{Al}_{2} \mathrm{O}_{3}$ films with only adventitious carbon on the surface that was removed after 2 minutes of $\mathrm{Ar}^{+}$sputtering; the remaining film showed $\mathrm{C}$ impurity of $\sim 1 \%$. Aluminum nitride attempts were performed using nitrogen plasma as a co-reagent. A saturating dose of TDMAA was achieved at a $4 \mathrm{~s}$ pulse, though this time after an $1800 \mathrm{sccm}$ 
bubbler pulse to attain a higher precursor vapor pressure needed to accommodate for tool geometry. Films appeared beige by eye, and XPS analysis confirmed the presence of the $\mathrm{Al}-\mathrm{N}$ expected peak after an initial 2 minute $\mathrm{Ar}^{+}$sputter. The films, however, did contain a large concentration of oxygen due to ambient exposure and storage, as well as a likely presence of oxygen during deposition, which caused the formation of an aluminum oxynitride. The carbon content of these films was very low, only $\sim 1 \%$ in the film itself after sputtering off adventitious carbon from postdeposition contamination. This $\mathrm{C}$ impurity level is lower than other aluminum nitride films deposited when using TMA as a precursor. It is likely that the aluminum-nitrogen bonds in TDMAA (as opposed to aluminum-carbon bonds in TMA) allowed for lower carbon contamination in film deposition. For this reason, it is worthwhile to pursue the study and use of tris(dimethylamido)aluminum(III) as a precursor for aluminum oxide and nitride films.

\subsubsection{Acknowledgements}

The authors would like to acknowledge Dr. Peter Gordon and Jianqun Wang at Carleton University for their assistance with SEM and EDS measurements, and Javier B. Giorgi at the University of Ottawa for the use of XPS facilities. 


\section{Chapter 3 - Part II}

\section{Epitaxial GaN using $\mathrm{Ga}\left(\mathrm{NMe}_{2}\right)_{3}$ and $\mathrm{NH}_{3}$}

\section{Plasma by Atomic Layer Deposition}

Modified from the original manuscript published as:

Rouf, P.; O’Brien, N. J.; Buttera, S. C.; Martinovic, I.; Bakhit, B.; Martinsson, E.;

Palisaitis, J.; Hsu, C.-W.; Pedersen, H. Epitaxial GaN Using Ga( $\left(\mathrm{NMe}_{2}\right)_{3}$ and $\mathrm{NH}_{3}$

Plasma by Atomic Layer Deposition. J. Mater. Chem. C 2020, 3, 1101-1134.

https://doi.org/10.1039/D0TC02085K. 


\subsubsection{Abstract}

Low temperature deposition of high-quality epitaxial GaN is crucial for its integration in electronic applications. Chemical vapor deposition at approximately $800{ }^{\circ} \mathrm{C}$ using $\mathrm{SiC}$ with an AlN buffer layer or nitridized sapphire as substrates is used to facilitate the GaN growth. Here, we present a low temperature atomic layer deposition (ALD) process using tris(dimethylamido)gallium(III) with $\mathrm{NH}_{3}$ plasma. The ALD process shows self-limiting behaviour between $130-250{ }^{\circ} \mathrm{C}$ with a growth rate of $1.4 \AA /$ cycle. The GaN films produced were crystalline on $\mathrm{Si}(100)$ at all deposition temperatures with a near stochiometric $\mathrm{Ga} / \mathrm{N}$ ratio with low carbon and oxygen impurities. When GaN was deposited on $4 \mathrm{H}-\mathrm{SiC}$, the films grew epitaxially without the need for an AlN buffer layer, which has never been reported before. The bandgap of the GaN films was measured to be $\sim 3.42 \mathrm{eV}$ and the fermi level showed that the GaN was unintentionally n-type doped. This study shows the potential of ALD for GaN-based electronic devices.

\subsubsection{Introduction}

Gallium nitride $(\mathrm{GaN})$ is a key material in microelectronics based on the group 13nitride materials. This is due to its favourable properties of wide and direct bandgap, high thermal stability, high breakdown field and good electron 
mobility. ${ }^{105-108}$ In high frequency electronics, GaN films are preferably deposited on $\mathrm{SiC}$ due to its high thermal conductivity. ${ }^{109}$ However, it is difficult to deposit GaN directly on SiC by CVD due to poor wetting of GaN on SiC. ${ }^{110}$ Therefore, an AlN buffer layer is used to facilitate GaN growth. ${ }^{111,112,113}$ Currently, thin films of electronic grade epitaxial GaN are deposited by chemical vapor deposition (CVD) processes using trimethylgallium (TMG) and ammonia $\left(\mathrm{NH}_{3}\right)$ at temperatures between $800-1000{ }^{\circ} \mathrm{C} .114$ The high deposition temperatures are required to obtain highly crystalline films but also to overcome the poorly suited precursor combination of TMG and $\mathrm{NH}_{3}$, which leads to high $\mathrm{N} / \mathrm{Ga}$ precursor ratios of $10^{3}$. They also hinder the deposition of highly conformal GaN films on topographically complex surfaces and on temperature sensitive materials. To meet these challenges, the low temperature time-resolved CVD route known as atomic layer deposition (ALD) has been studied for GaN. Deposition of GaN by ALD has been achieved using TMG or triethylgallium (TEG) with $\mathrm{N}_{2} / \mathrm{H}_{2}$ plasma ${ }^{115}$, $\mathrm{NH}_{3}$ plasma ${ }^{115,116}$ or thermally with $\mathrm{NH}_{3}{ }^{117}$. However, these processes suffer non-stoichiometric Ga/N ratios and high carbon and oxygen impurities. Additionally, thermal ALD with TMG and $\mathrm{NH}_{3}$ requires deposition temperatures of $400{ }^{\circ} \mathrm{C}$, which is relatively high for an ALD process. ${ }^{117}$ Similar issues has been reported for ALD of GaN using TEG, where impurities of $\mathrm{C}$ and $\mathrm{O}$ were the primary issue. ${ }^{118,119}$. ALD routes using $\mathrm{GaCl}$ and $\mathrm{GaCl}_{3}$ together with $\mathrm{NH}_{3}$ deposited at high temperature $\left(>400{ }^{\circ} \mathrm{C}\right)$ have also been investigated, but render films with $\mathrm{Cl}$ impurities. ${ }^{104,120}$ 
The disadvantage of TMG and TEG for ALD of GaN is their strong M-C bonds, which makes it difficult to remove all of their ligands from the deposited precursor at low temperatures. ${ }^{121}$ Amides $\left(\mathrm{M}-\mathrm{NR}_{2}\right)$ have more reactive and desirable M-N bonds, which would improve the surface chemistry during the $\mathrm{NH}_{3}$ precursor pulse. Herein, we report low temperature deposition of GaN using tris(dimethylamido)gallium(III), $\left(\mathrm{Ga}\left(\mathrm{N}\left(\mathrm{CH}_{3}\right)_{2}\right)_{3}\right.$ 4, with $\mathrm{NH}_{3}$ plasma by ALD. Precursor 1 has been previously used for ALD of $\mathrm{Ga}_{2} \mathrm{O}_{3}{ }^{122,123}$ and $\mathrm{Ga}_{2} \mathrm{~S}_{3}{ }^{124}$ and CVD of GaN, ${ }^{125}$ but not for ALD of GaN. Notably, all previous reports using 4 has resulted in amorphous films at all deposition temperatures. ${ }^{122,124,125}$ We show that 4 displayed self-limiting behaviour in a temperature range from $130-250{ }^{\circ} \mathrm{C}$ on $\mathrm{Si}(100)$. Deposition of $\mathrm{GaN}$ on $4 \mathrm{H}-\mathrm{SiC}(0001)$ rendered epitaxial films with near stochiometric composition and very low impurity levels of carbon and oxygen. To the best of our knowledge, this is the first report of GaN grown epitaxially directly on SiC substrate.

\subsubsection{Experimental}

\section{Precursor Synthesis}

The reaction and manipulations were carried out under a $\mathrm{N}_{2}$ atmosphere on a Schlenk line using Schlenk air-free techniques and in a Glovebox-Systemtechnik dry box. All anhydrous solvents were purchased from Sigma-Aldrich ${ }^{\mathrm{TM}}$ and further 
dried with $4 \AA$ molecular sieves. All NMR spectra were measured with an Oxford Varian 300 and spectrometer. Solvents peaks were used as an internal standard for the ${ }^{1} \mathrm{H}$ NMR (300 MHz) spectrum. The melting point was determined in a capillary sealed under $\mathrm{N}_{2}$ with a Stuarts SMP10 melting point apparatus and is uncorrected. $\mathrm{GaCl}_{3} \quad(99.99 \%)$ was purchased from ACROS-Organics ${ }^{\mathrm{TM}}$ and lithium dimethylamide (95\%) from Sigma-Aldrich ${ }^{\top M}$, and both were used without further purification.

\section{Synthesis of tris(dimethylamido)gallium(III) dimer (4)}

Tris(dimethylamido)gallium(III) dimer 4 was synthesised using a modified literature procedure (Figure 3.8). ${ }^{126} \mathrm{~A}$ room temperature suspension of lithium dimethylamide (5.2 g, $102 \mathrm{mmol})$ in $n$-hexanes $(150 \mathrm{~mL})$ was slowly added to a $20{ }^{\circ} \mathrm{C}$ solution of $\mathrm{GaCl}_{3}(6.0 \mathrm{~g}, 34.1 \mathrm{mmol})$ in $n$-hexanes $(100 \mathrm{~mL})$ via cannula and the mixture was stirred at this temperature for $1 \mathrm{~h}$. The reaction was slowly warmed to room temperature and stirred for a further $16 \mathrm{~h}$. The reaction mixture was filtered through a pad of Celite and concentrated under reduced pressure to give a solid. The solid was purified by recrystallisation from $\mathrm{Et}_{2} \mathrm{O}$ at $-35{ }^{\circ} \mathrm{C}$ to give the compound 4 as a solid $(4.61 \mathrm{~g}, 63 \%)$.

4: Colourless solid, m.p. $101-104{ }^{\circ} \mathrm{C} .{ }^{1} \mathrm{H}$ NMR $\left(300 \mathrm{MHz}, \mathrm{C}_{6} \mathrm{D}_{6}\right) \delta 2.34(\mathrm{~s}, 12 \mathrm{H}, \mathrm{p}-$ $\mathrm{NMe} 2), 2.68\left(24 \mathrm{H}, \mathrm{\mu}-\mathrm{N} M e_{2}\right)$. 

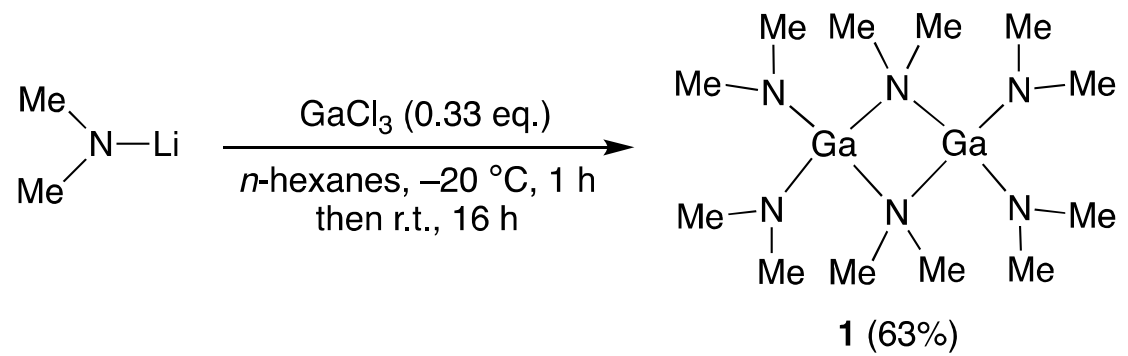

Figure 3.8: Synthesis of tris(dimethylamido)gallium(III) 4.

\section{Thermogravimetric Analysis and Differential Scanning}

\section{Calorimetry}

The volatilization curve was collected using a TA Instruments thermogravimetric analysis Q500 tool. The ramp experiment of $\mathbf{4}$ was undertaken inside a nitrogen glovebox in a tared aluminum pan loaded with $18 \mathrm{mg}$ of $\mathbf{4}$. The furnace was heated at a rate of $10{ }^{\circ} \mathrm{C} / \mathrm{min}$ to $400{ }^{\circ} \mathrm{C}$ with a maintained nitrogen flow rate of $60 \mathrm{sccm}$. The differential scanning calorimetry (DSC) measurement for $\mathbf{4}$ was performed on a TA Instruments DSC Q10 tool. The sample was prepared in a sealed aluminum pans in a $\mathrm{N}_{2}$ filled glovebox and weighed approximately $0.2 \mathrm{mg}$. The experiments heated the sample of 4 and a blank reference pan at a rate of $10{ }^{\circ} \mathrm{C} / \mathrm{min}$ to $400{ }^{\circ} \mathrm{C}$.

\section{Film Deposition}

The films were deposited in a Picosun R-200 ALD system equipped with a Litmas

Remote Inductively Coupled Plasma Source. The system used a base pressure of $400 \mathrm{~Pa}$ with continuous $\mathrm{N}_{2}(99,999 \%$, further purified with a getter filter to remove 
moisture) flow through the deposition chamber. The $\mathrm{Si}(100)$ and $4 \mathrm{H}-\mathrm{SiC}(0002)$ substrates were cut into $1.5 \times 1.5 \mathrm{~cm}$ pieces and $\mathrm{Si}(100)$ was used without further cleaning whilst $4 \mathrm{H}-\mathrm{SiC}$ was cleaned with RCA-1 (solution of 1 part $\mathrm{H}_{2} \mathrm{O}_{2}(30 \%), 1$ part $\mathrm{NH}_{3}(25 \%)$ and 5 parts $\left.\mathrm{H}_{2} \mathrm{O}\right)$ and $\mathrm{RCA}-2\left(1\right.$ part $\mathrm{H}_{2} \mathrm{O}_{2}(30 \%), 1$ part $\mathrm{HCl}(37 \%)$ and 6 parts $\left.\mathrm{H}_{2} \mathrm{O}\right)^{127}$ solutions to remove organic and inorganic contaminants on the surface prior to deposition. The substrates were loaded into the reactor onto a heated substrate holder and the system was heated to $450{ }^{\circ} \mathrm{C}$ for 120 minutes before each run with a continuous $\mathrm{N}_{2}$ flow $(300 \mathrm{sccm})$ to minimize the oxygen content in the chamber. Approximately $500 \mathrm{mg}$ of $\mathbf{4}$ was placed in a glass vial in a stainlesssteel bubbler without a dip-tube for incoming carrier gas. The temperature of the bubbler was set at $120{ }^{\circ} \mathrm{C}$ with a $\mathrm{N}_{2}$ flow of $100 \mathrm{sccm}$ to aid transporting the precursor vapor into the deposition chamber. A $10 \mathrm{~s} \mathrm{~N}_{2}$ purge was used after the pulse of 4 . The $\mathrm{NH}_{3}$ (AGA/Linde, $99.999 \%$ ) plasma used as the nitrogen source was an $\operatorname{Ar}\left(99.999 \%\right.$, further purified with a getter filter to remove moisture) $/ \mathrm{NH}_{3}$ $(100 / 75 \mathrm{sccm})$ mixture, ignited using a plasma power of $2800 \mathrm{~W}$. These plasma parameters were previously investigated to be optimal for our reactor and deposition of nitrides. ${ }^{128}$ A plasma pulse of $9 \mathrm{~s}$ followed by a $10 \mathrm{~s}$ purge was used with the above parameters unless otherwise stated. The thickness of the GaN film was $70 \mathrm{~nm}$ unless otherwise stated. 


\section{Characterization}

The crystallinity of deposited films was studied using a PANalytical EMPYREAN MRD XRD with a Cu-anode $\mathrm{x}$-ray tube and 5-axis (x-y-z-v-u) sample stage both in $\theta-2 \theta$ and grazing incidence (GIXRD) configuration. Strain in the films was estimated from the GIXRD measurement for each diffraction peak values of $\sin ^{2} \psi$ and $\varepsilon$ using the software X'Pert Stress. PANalytical X'Pert PRO with a Cu-anode tube and Bragg-Brentano HD optics was used for $\theta-2 \theta$ measurement and in X-ray Reflectivity (XRR) mode to measure the film thickness. The software PANalytical X'Pert reflectivity was used to fit the XRR data using a two-layer model of the GaN film and the substrate. A LEO 1550 Scanning electron microscope (SEM) with an acceleration energy of $10-20 \mathrm{kV}$ was used to characterise the morphology of the film. The film composition and chemical bonding environments of the atoms in the film were characterized with a Kratos AXIS Ultra DLD X-ray photoelectron spectroscopy (XPS) equipped with Ar $(0.5 \mathrm{keV})$ sputtering source. CasaXPS software was used to evaluate the data. Gaussian-Laurentius functions and Shirley background were used to fit the experimental XPS data. The absorption spectroscopy measurements were conducted using a custom fibre optical setup consisting of a light source (Ocean Optics DH-2000-BAL), a detector (Avantes AvaSpec-Dual) and a bifurcated optical fibre (Ocean Optics BIFBORO-2-1000). An absorption spectrum for the GaN film was collected using a custom software 
based on LabView (National Instruments) with a Si (100) substrate used as a reference.

Elemental composition of the films was obtained using Rutherford backscattering spectrometry (RBS) and time-of-flight elastic recoil detection analyses (ToF-ERDA). The measurements were carried out in a 5-MV NEC-5SDH2 pelletron tandem accelerator. $2 \mathrm{MeV}^{4} \mathrm{He}^{+}$ions were employed for $\mathrm{RBS}$ and detected in a scattering angle of $170^{\circ}$. Two different geometries, azimuth angle $5^{\circ}+$ tilt angle $2^{\circ}$ and azimuth angle $40^{\circ}+$ tilt angle $2^{\circ}$, were chosen in order to minimize channelling effects. In addition, more suppression of the probable channelling effects was undertaken by multiple small random-angular movements around the equilibrium angles within a range of $2^{\circ}$. RBS spectra were fitted by SIMNRA 7.02 code $^{129}$ with an $\sim 1 \%$ statistic uncertainty to determine elemental compositions. Recoils, in ToF-ERDA, were detected at $45^{\circ}$ angle between the primary beam and a ToF-E detector telescope in a gas ionization chamber (GIC) using a $36-\mathrm{MeV}{ }^{127} \mathrm{I}^{8+}$ beam incident at $67.5^{\circ}$ with respect to the sample surface normal. The ToF-E detector telescope consisted of two circular carbon foils with 8 and $5 \mu \mathrm{g} / \mathrm{cm}^{2}$ thicknesses, $6 \mathrm{~mm}$ radius, a $0.05-\mathrm{msr}$ solid angle $(\Delta \Omega)$, and a flight distance of $425 \mathrm{~mm}$ between the foils. Utilizing a ToF-GIC setup provides the system with a good energy resolution and enhanced ion species separation in terms of mass and energy. ${ }^{130}$ Average elemental compositions were also obtained from 
ToF-ERDA time-energy coincidence spectra using two different software packages, CONTES ${ }^{131}$ and Potku. ${ }^{132}$ Systematic uncertainties of the experiment, discussed in more detail elsewhere ${ }^{133}$ in particular for light elements, were estimated to be a maximum of $5-10 \%$, whereas statistic uncertainties arisen from the number of experimental counts were $<2.3 \%$. However, the relative elemental concentrations were obtained with higher accuracy. ${ }^{134,135}$ The stopping power data required for both RBS and ERDA simulations was retrieved from SRIM2013 code. ${ }^{136}$

Cross-sectional transmission electron microscope (TEM) samples were prepared by the traditional sandwich approach, which includes sample cutting, gluing, polishing and ion milling. A Gatan Precision Ion Polishing System Model 691 operated at $5 \mathrm{kV}$ and $40 \mathrm{~mA}$, with an Ar ion source, was used to make the samples electron transparent. Scanning transmission electron microscopy (STEM) and selective area electron diffraction (SAED) characterization were performed using the Linköping double Cs corrected FEI Titan ${ }^{3}$ 60-300 operated at $300 \mathrm{kV}$.

\subsubsection{Results and Discussion}

Tris(dimethylamido)gallium(III) dimer $\mathbf{4}$ has been previously used in ALD studies, ${ }^{122,123}$ however its thermal properties have not been reported as far as we know. Thermogravimetric analysis (TGA) showed $\mathbf{4}$ evaporated in a single step from $140-230{ }^{\circ} \mathrm{C}$, with only $5 \%$ of residual mass (Figure 3.9). The 1 torr vapour 
pressure of 4 was shown to occur at $109 \pm 5{ }^{\circ} \mathrm{C}$ its $\Delta \mathrm{H}$ of vaporization was 59.5 $\mathrm{kJ} \mathrm{mol}^{-1}$ (Figure 3.16). Differential scanning calorimetry (DSC) showed an endothermic peak at $103{ }^{\circ} \mathrm{C}$ and a small broad exothermic event with a peak at $193{ }^{\circ} \mathrm{C}$. The endothermic event peak corresponds to the melting point of 4 (103$106{ }^{\circ} \mathrm{C}$ ), which matches our observed DSC value, whilst the exothermic event indicates high temperature decomposition. These results show that $\mathbf{4}$ is a thermally stable precursor and has favourable properties for use in ALD of GaN.

(a)

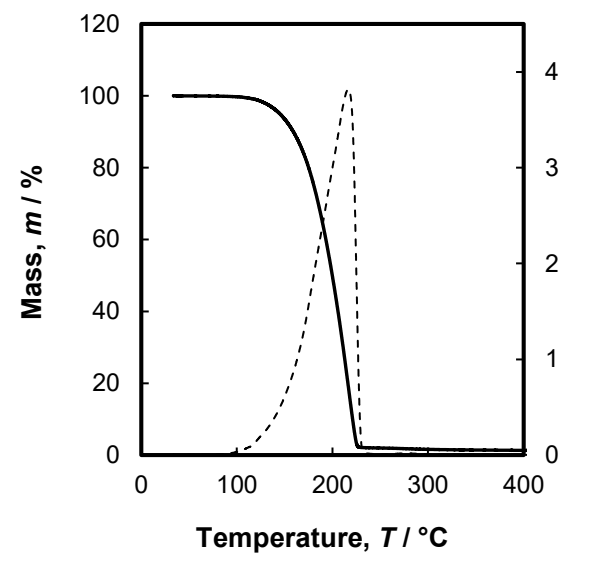

(b)

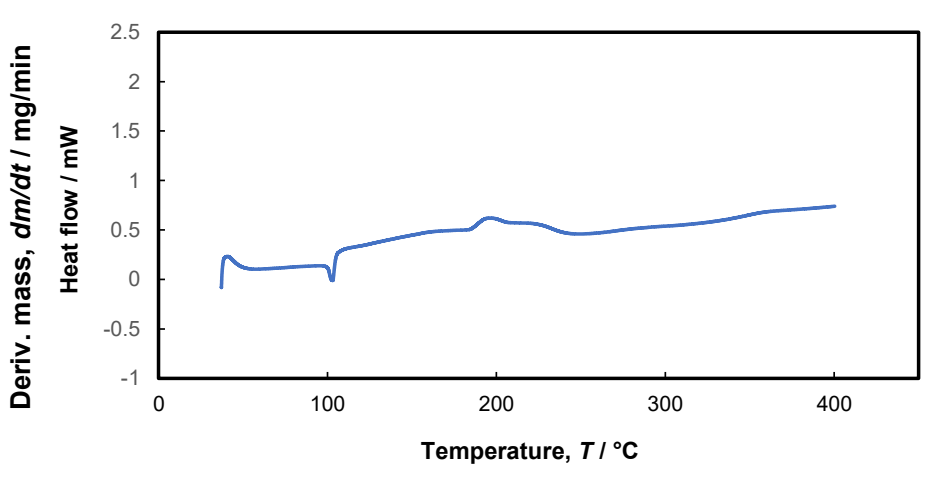

Figure 3.9: a) Thermogravimetric analysis and b) differential scanning calorimetry of 4 .

To study if 4 could be used in self-limiting deposition of GaN, ALD experiments on Si (100) substrates were undertaken using varied pulse times for $\mathbf{4}$ and $\mathrm{NH}_{3}$ plasma whilst the temperature was maintained at $200{ }^{\circ} \mathrm{C}$. The GaN growth per cycle (GPC) saturates at $1.4 \AA$ /cycle when the pulse time of 4 is $4 \mathrm{~s}$ or 
longer (Figure 3.10a). The GPC saturates at the same value for $\mathrm{NH}_{3}$ plasma when pulse times of $6 \mathrm{~s}$ or longer were used. This is indicative of a self-limiting growth and surface chemistry between 4 and $\mathrm{NH}_{3}$ plasma. The growth per cycle was constant between $130-250{ }^{\circ} \mathrm{C}$ when using a $4 \mathrm{~s}$ pulse time for 4 and $9 \mathrm{~s} \mathrm{NH}_{3}$ plasma pulse time (Figure 3.10b). As the bubbler temperature was set to $120{ }^{\circ} \mathrm{C}$, lower deposition temperature was not conducted due to the risk of condensation of $\mathbf{4}$ on the substrate. Reactor temperatures of $\geq 250{ }^{\circ} \mathrm{C}$ resulted in a decreased GPC, indicating desorption of the precursor from the substrate.

This GPC is roughly 3-5 times that of previous reports of GaN plasma processes with TMG/TEG. ${ }^{115,116,118}$ It can be noted here that the wurtzite GaN lattice has a $c$ lattice constant of $5.182 \AA$. To obtain such a unit cell, three monolayers are required (Ga source, N source followed by Ga source) which corresponds to 1.5 ALD cycles and therefore the theoretical maximum growth per cycle is approximately $3.45 \AA$ /cycle. The growth of $1.4 \AA$ /cycle obtained with the $\mathrm{M}-\mathrm{N}$ bonded precursor 4 is lower than the theoretical growth making it reasonable despite its higher growth rate compared to other GaN processes.

The films deposited in the temperature window with constant GPC rendered crystalline GaN with a preferred (002) orientation on Si(100) (Figure 3.11a). The intensity of the XRD peaks, which is especially clear from the GIXRD measurements (Figure 3.11b), increases with higher deposition temperature 
suggesting that film crystallinity increases with temperature. The (002) plane is present in both the $\theta-2 \theta$ XRD and the GIXRD indicating that grains tilted with respect to the substrate normal are present in the film. The stress and strain in the $70 \mathrm{~nm}$ GaN films were estimated from the GIXRD measurement by a $\varepsilon-\sin ^{2} \psi$ plot, Figure 3.11c. The positive slope of the fitted line to the plotted data is indicative of tensile strain of the film. The strain was obtained from the slope (7475.61 ppm) and gives a value of 0.00747561 . This corresponds to roughly $0.75 \%$ of tensile strain. To calculate the stress in the film, the Young's modulus (E) and the Poisson's ratio (v) for the GaN was used (Equation 1).

$$
\sigma=\frac{E * \varepsilon}{1+v}
$$

The Youngs's modulus and Poisson's ratio of GaN have been determined from previous studies to be $325.3 \mathrm{GPa}$ and 0.25 , respectively. ${ }^{137}$ This gives a tensile stress of about $1.95 \mathrm{GPa}$ for the GaN thin film on a Si substrate. Tensile strain and stress in the GaN film is expected due to the large difference in lattice constant and crystal structure of cubic Si to hexagonal GaN.

Top-view SEM analysis showed that the films deposited at $250{ }^{\circ} \mathrm{C}$ is composed of many small crystalline grains with little variation in grain size (Figure 3.11d). The little variation in grain size indicates that the film grows in a layer-bylayer fashion without any observable secondary nucleation. 
(a)

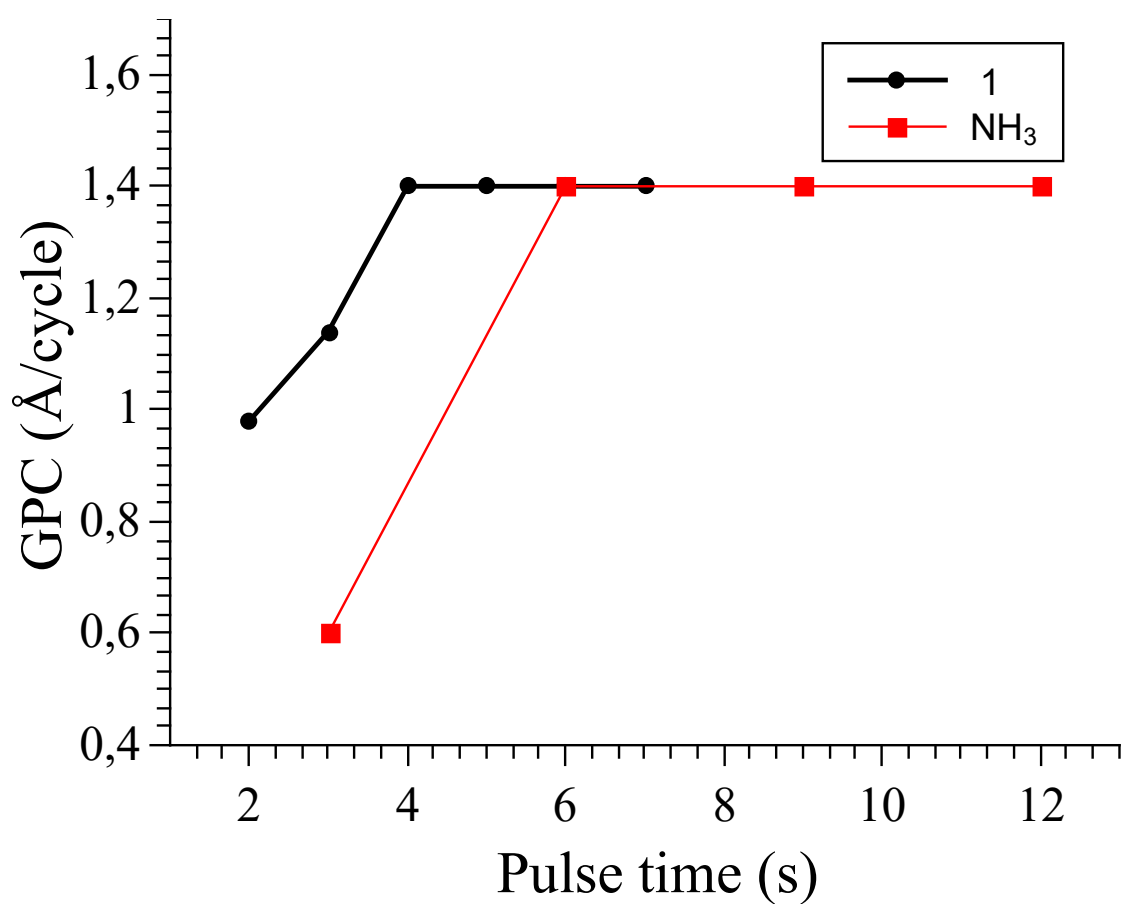

(b)

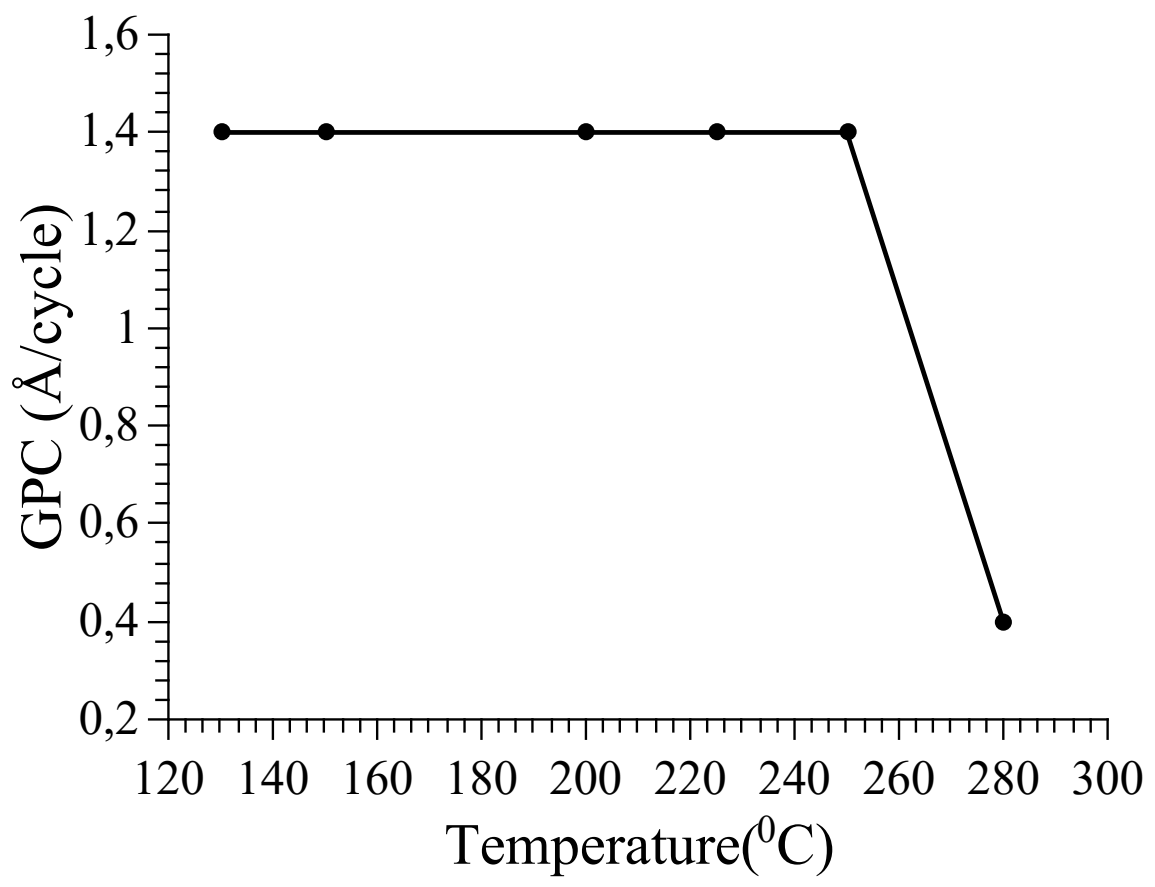

Figure 3.10: a) The saturation curves of 4 (black circles) with $9 \mathrm{~s} \mathrm{NH}_{3}$ plasma pulse and $\mathrm{NH}_{3}$ plasma (red squares) with $4 \mathrm{~s}$ pulse of 4 deposited at $220{ }^{\circ} \mathrm{C}$. b) The GPC dependence on temperature using $9 \mathrm{~s}$ pulses of $\mathrm{NH}_{3}$ plasma and $4 \mathrm{~s}$ pulses of 4 . 
(a)

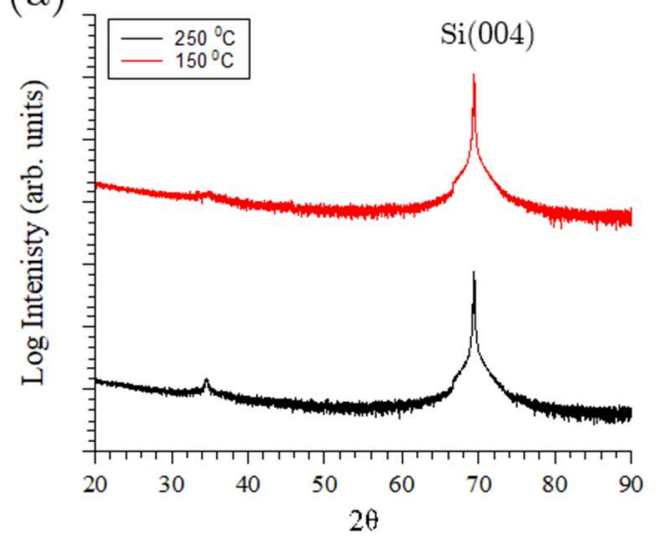

(c)

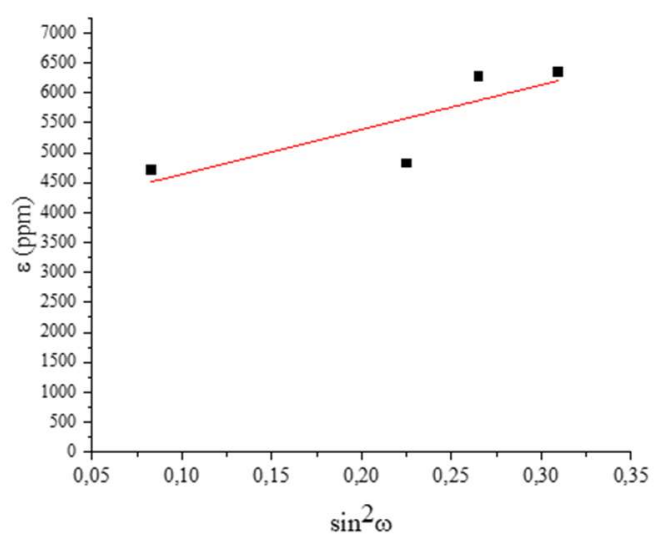

(b)

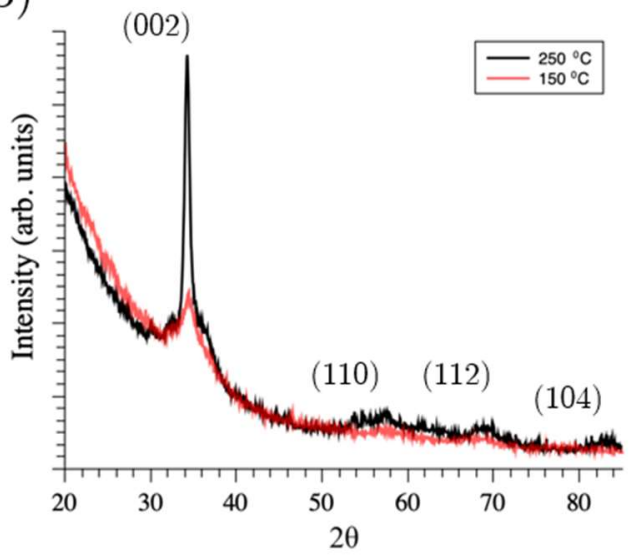

(d)

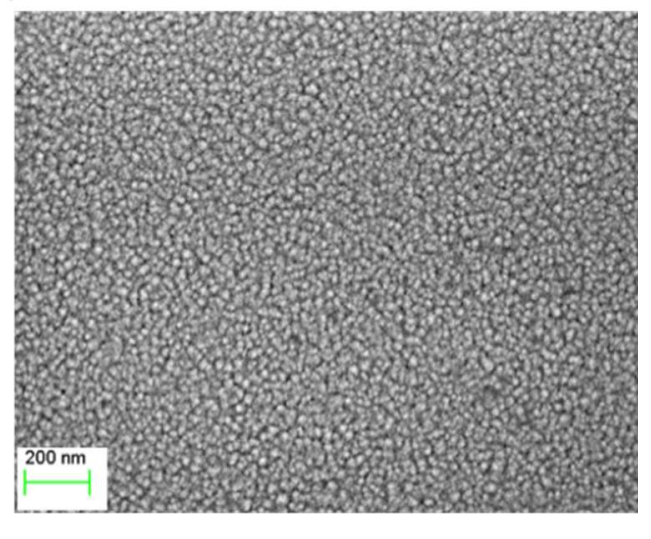

Figure 3.11: a) XRD of the films on $\mathrm{Si}(100)$ showing $\mathrm{GaN}(002)$ peak deposited at $150{ }^{\circ} \mathrm{C}$ (red line) and $250{ }^{\circ} \mathrm{C}$ (black line) with $9 \mathrm{~s} \mathrm{NH}_{3}$ plasma pulse and $4 \mathrm{~s}$ pulse of 4 with a thickness of approximately $70 \mathrm{~nm}$. b) GIXRD of the films on $\operatorname{Si}(100)$ with $4 \mathrm{~s}$ pulse of $4,9 \mathrm{~s} \mathrm{NH}_{3}$ plasma pulse deposited at $150{ }^{\circ} \mathrm{C}$ (red line) and 250 ${ }^{\circ} \mathrm{C}$ (black line) showing GaN(002) peak with a thickness of approximately $70 \mathrm{~nm}$. c) $\varepsilon-\sin ^{2} \psi$ plot from GIXRD measurement on $\mathrm{Si}(100)$ with $4 \mathrm{~s}$ of 4 and $9 \mathrm{~s} \mathrm{NH}_{3}$ plasma deposited at $250{ }^{\circ} \mathrm{C}$ with a thickness of approximately $70 \mathrm{~nm}$. d) Top-view $\mathrm{SEM}$ of the film on $\mathrm{Si}$ (100) with $4 \mathrm{~s}$ pulse of 4 and $9 \mathrm{~s} \mathrm{NH} 3$ pulse deposited at 250 ${ }^{\circ} \mathrm{C}$ with a thickness of approximately $70 \mathrm{~nm}$.

The chemical bonding environment and atomic composition in the GaN films deposited at $250{ }^{\circ} \mathrm{C}$ were analysed by XPS (Figure 3.12). High resolution XPS spectra were used to analyse the chemical environment of the Ga $2 \mathrm{p}_{3 / 2}$ and $\mathrm{N} 1 \mathrm{~s}$ 
regions. Two peaks were used to obtain a good fit for the Ga $2 \mathrm{p}_{3 / 2}$ at $1117.5 \mathrm{eV}$ and $1119.4 \mathrm{eV}$, which were attributed to $\mathrm{Ga}-\mathrm{N}$ and $\mathrm{Ga}-\mathrm{O}$ bonds, respectively (Figure 3.12a). Three peaks were used to fit the N 1s at $397.1 \mathrm{eV}, 394.8 \mathrm{eV}$ and $392.7 \mathrm{eV}$, which were assigned to $\mathrm{N}-\mathrm{Ga}$ and two Ga Auger peaks, respectively (Figure 3.12b). This is in accordance with previous XPS measurements on GaN thin films. ${ }^{138,139,115,117}, 140$ After sputter cleaning the film surface, XPS measurements gave an initial overall composition of 25.0 at.\% Ga, 74.5 at.\% N, 0.5 at.\% $\mathrm{O}$ with no detectable $\mathrm{C}$. It should be noted that the $\mathrm{N}$ 1s peak overlaps with Ga Auger peaks, which results in a broad N 1s peak and an overestimate of the $\mathrm{N}$ content. To obtain the Ga/ $\mathrm{N}$ ratio in the film without Ga Auger interference, the Auger peaks were subtracted from the contribution to the $\mathrm{N}$ content. The NGa peak contribution is $33 \%$ of the overall $\mathrm{N}$ content, which gives 24.6 at.\% of $\mathrm{N}$ that is attributed to the actual $\mathrm{N}$ content of the film. This gives a corrected Ga/N ratio of 1.02 , which is close to stochiometric GaN. The high resolution XPS peak of Ga $2 \mathrm{p}_{3 / 2}$ can be fitted with contributions from $\mathrm{Ga}-\mathrm{N}$ and $\mathrm{Ga}-\mathrm{O}$ bonds while the high resolution XPS $\mathrm{N}$ 1s peak cannot be fitted with a $\mathrm{N}-\mathrm{O}$ peak, suggesting that all oxygen in the film is bonded to Ga. If oxygen is subtracted from Ga $2 \mathrm{p}_{3 / 2}$ spectra and only the $\mathrm{Ga}-\mathrm{N}$ and $\mathrm{N}-\mathrm{Ga}$ bonds are considered, a near stoichiometric Ga/N ratio of 0.99 is obtained. To investigate if the subtraction of the Ga Auger peaks from the N 1s XPS region is a good strategy, RBS combined with ERDA was conducted on the GaN film. The RBS/ERDA measurement showed that the GaN 
film contained 45.7 at.\% Ga, 47.2 at.\% N, 2.8 at.\% C, 3.1 at.\% $\mathrm{O}$ and 1.2 at.\% H giving a $\mathrm{Ga} / \mathrm{N}$ ratio of 0.97 , which indicates a slightly under-stochiometric film composition. This shows that even when subtracting the Ga Auger peak from the $\mathrm{N}$ 1s XPS region, the film composition and $\mathrm{Ga} / \mathrm{N}$ ratio is not reliable and other techniques are required to determine an accurate atomic content. In addition, C impurities were not detected by XPS after clean sputtering whilst RBS/ERDA showed $\mathrm{C}$ present in the film, showing the latter is preferred for detection of impurities. In addition, sputtering was used in the XPS measurement, which could cause preferential sputtering and change the composition and impurity levels in the film. Nonetheless, XPS is highly important for accessing the chemical environment of each element.
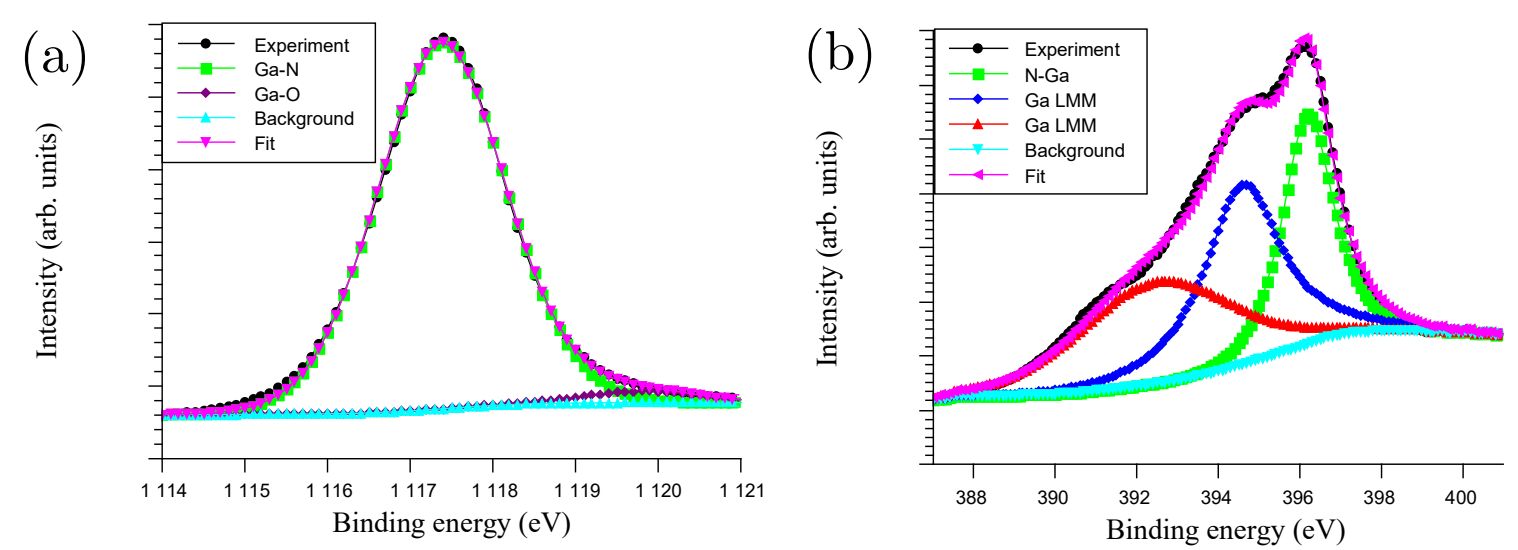

Figure 3.12: HR-XPS of Ga 2p (a) and N $1 \mathrm{~s}$ (b) with $4 \mathrm{~s}$ of 4 and $9 \mathrm{~s} \mathrm{NH}_{3}$ plasma pulse deposited at $250{ }^{\circ} \mathrm{C}$ with a thickness of $70 \mathrm{~nm}$.

An absorption measurement was conducted on the GaN film and the data was used to construct a Tauc plot to calculate the bandgap. The optical bandgap 
for a direct bandgap material is expressed by equation 2. Extrapolating the linear part in the Tauc plot and setting $(\alpha \mathrm{hv})^{2}$ to 0 gives the band gap.

$$
\alpha h v=A\left(h v-E_{g}\right)^{\frac{1}{2}}
$$

The Tauc plot for GaN films deposited at $250{ }^{\circ} \mathrm{C}$ gave a band gap value of approximately $3.42 \mathrm{eV}$ (Figure $3.13 \mathrm{a}$ ). This is close to the previously reported single crystalline GaN value of $3.40 \mathrm{eV} .{ }^{109}$ The valence band (VB) spectrum obtained from XPS has two distinct features, labelled A and B in Figure 3.13b, which correspond to Ga $4 \mathrm{p}-\mathrm{N} 2 \mathrm{p}$ and $\mathrm{Ga} 4 \mathrm{p}-\mathrm{N}$ 4s hybridized orbitals, respectively. These features are in line with previous studies on GaN thin films. ${ }^{141,142}$ The VB is near the Fermi level $\left(\mathrm{E}_{\mathrm{F}}\right)$ obtained by XPS (Figure 3.13c). By extrapolating the linear part of the leading edge and the baseline, the valence band maxima (VBM) could be obtained. It was found that the VBM lies approximately $2.20 \mathrm{eV}$ below the surface Fermi level and is in line with a previous GaN study deposited with by MBE. ${ }^{141}$ In combination with the estimated band gap of $3.42 \mathrm{eV}$, this result indicates that the Fermi level is not positioned in the middle of the bandgap, but closer to the conduction band (CB) instead of the VB (Figure 3.13d). This shift of the Fermi level indicates that the deposited GaN is n-type doped. This is expected as GaN normally shows n-type behaviour due to impurities and defects in the film such as oxygen and nitrogen vacancies. ${ }^{143,144,145}$ 
(a)

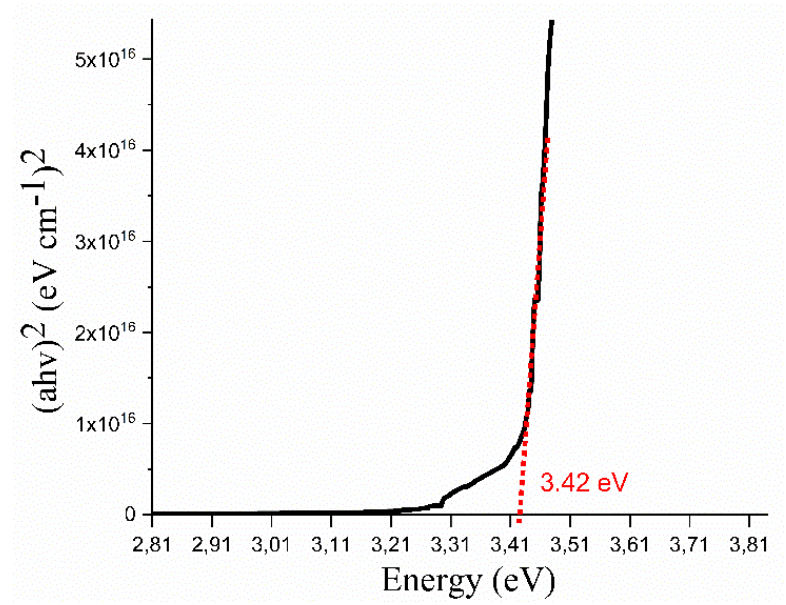

(c)

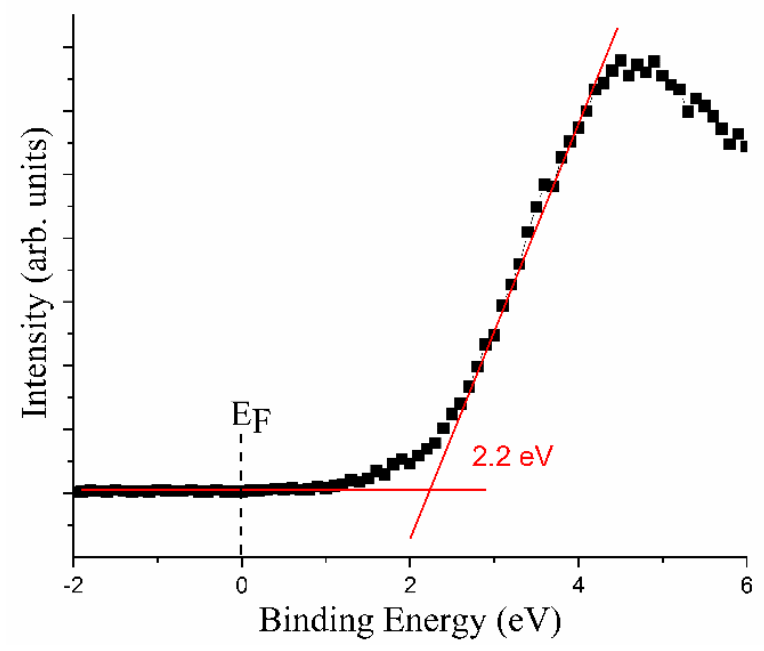

(b)

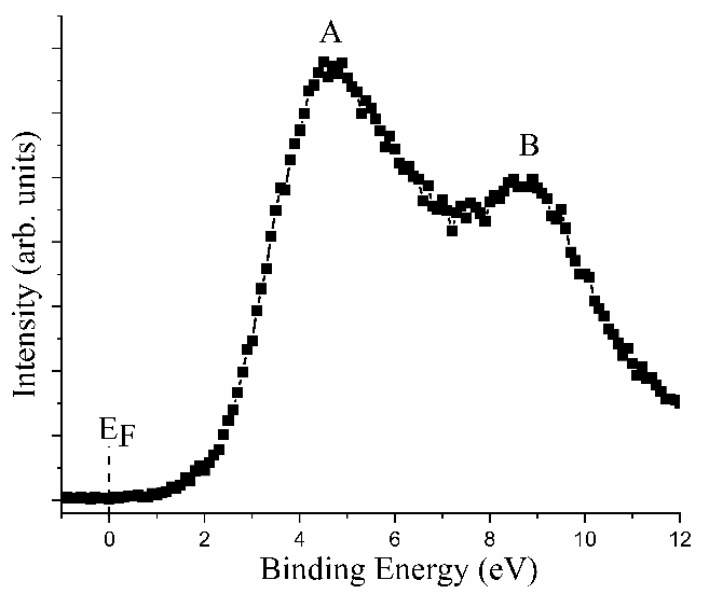

(d)

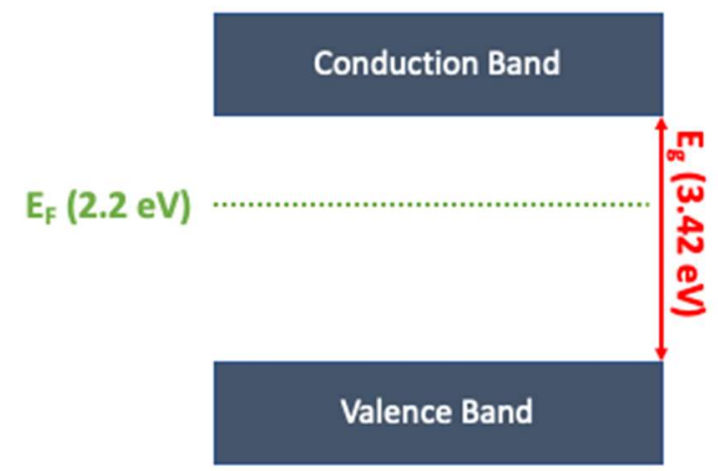

Figure 3.13: a) The absorption spectrum for the GaN deposited at $250{ }^{\circ} \mathrm{C}$ with a 4 s pulse of 4 and $9 \mathrm{~s} \mathrm{NH}_{3}$ plasma pulse on $\mathrm{Si}$ (100) with a thickness of approximately $70 \mathrm{~nm}$. b) The valence band spectrum of the GaN film with a thickness of approximately 70nm obtained by XPS. c) The near Fermi level $\left(\mathrm{E}_{\mathrm{F}}\right)$ region together with the valence band maxima obtained from extrapolating the baseline with the linear fit of the XPS spectrum. d) Schematic illustration of the energy band diagram for GaN deposited at $250{ }^{\circ} \mathrm{C}$. 
By replacing the $\mathrm{Si}(100)$ substrate with $4 \mathrm{H}-\mathrm{SiC}$ (0001), which is a more suitable substrate for GaN with respect to the lattice mismatch and crystal structure, XRD in $\theta-2 \theta$ geometry showed only the GaN (0002) and (0004) peaks together with the substrate peaks. This indicates that growth occurred only in the c-direction (Figure 3.14a). The GaN film was deposited at $250{ }^{\circ} \mathrm{C}$ using a 4 s pulse of 4 and $9 \mathrm{~s} \mathrm{NH}_{3}$ plasma pulse. GIXRD of the GaN film grown on $\mathrm{SiC}$ showed no peaks, indicating no tilted grains present in the film (Figure 3.14b). Pole figures were constructed to investigate the epitaxial relationship between the film and the substrate (Figure 3.14c and 3.14d). The GaN (1013) plane was used due to large difference in the psi angle between $\mathrm{GaN}$ and $\mathrm{SiC}$, making it possible to distinguish between the $\mathrm{GaN}$ and $\mathrm{SiC}$ poles. The pole figure showed six poles, representing the hexagonal GaN lattice with its six-fold symmetry (Figure 3.14c). The SiC (10̄12) plane show six poles, representing its hexagonal six-fold symmetry (Figure 3.14d). These pole figures show that the hexagonal GaN crystals have grown exactly on top of the SiC crystals, confirming that epitaxial GaN has been grown directly on $\mathrm{SiC}$ substrate. The epitaxial relationship between the film and the substrate is $\operatorname{GaN}(0002) \| \mathrm{SiC}(0004)$ and $\mathrm{GaN}(10 \overline{1} 3) \| \mathrm{SiC}(10 \overline{1} 2)$. It is worth noting that no AlN interlayer was used to aid the growth of $\mathrm{GaN}$ on $\mathrm{SiC}$, which is required for CVD of GaN on SiC. ${ }^{111,146}$ This opens up the possibility for deposition of GaN on transistor structures without the need of a buffer layer between $\mathrm{SiC}$ and $\mathrm{GaN}$. 
Top-view SEM of the GaN film on $\mathrm{SiC}$ deposited at $250{ }^{\circ} \mathrm{C}$ was composed of larger grains compared to GaN on Si substrate (Figure 3.18).

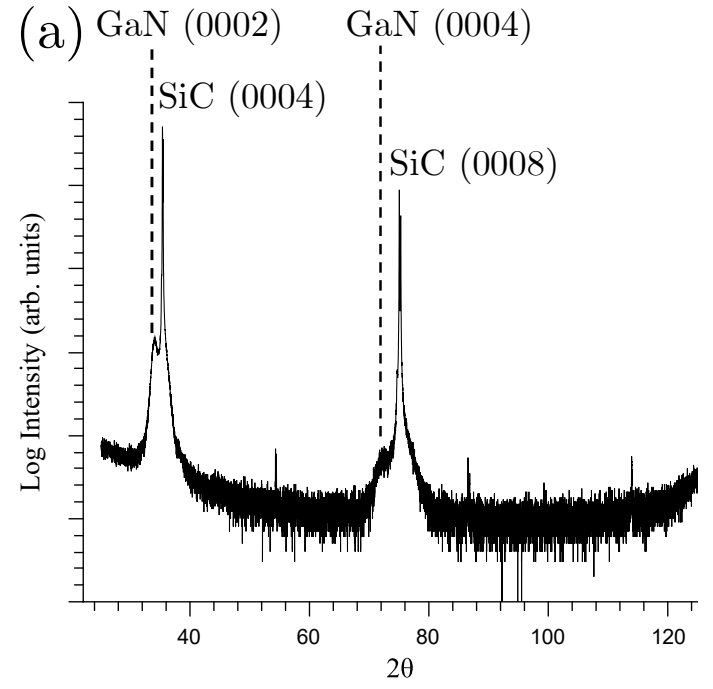

(c)

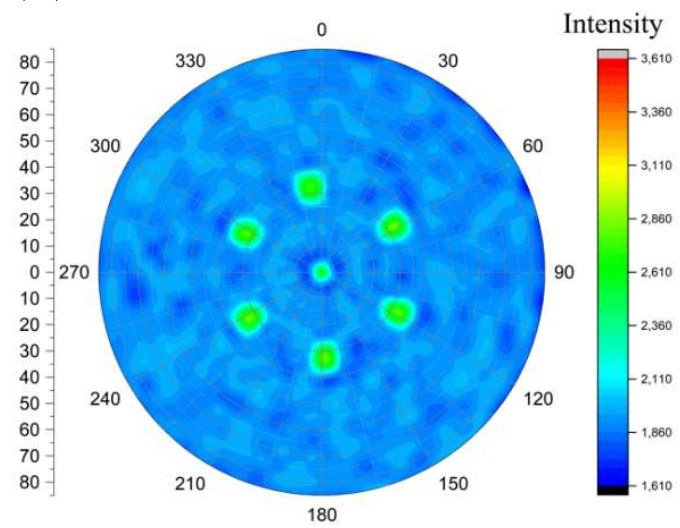

(b)

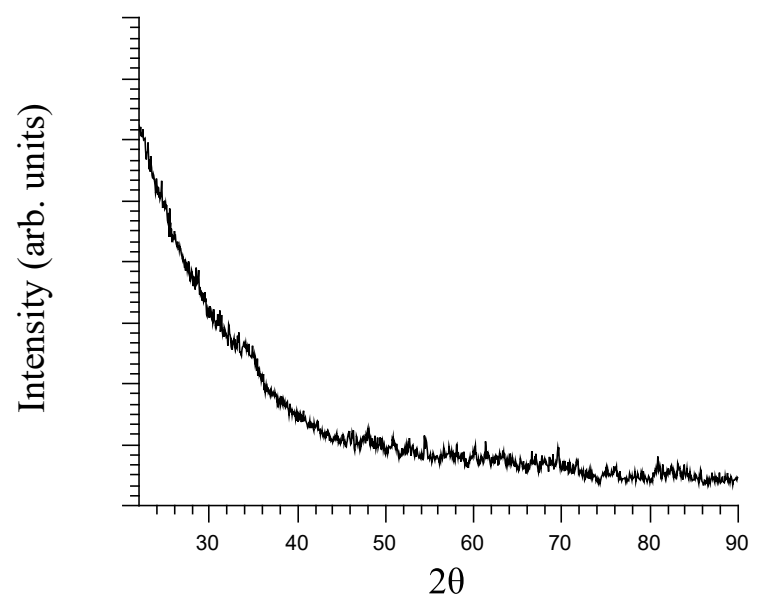

(d)

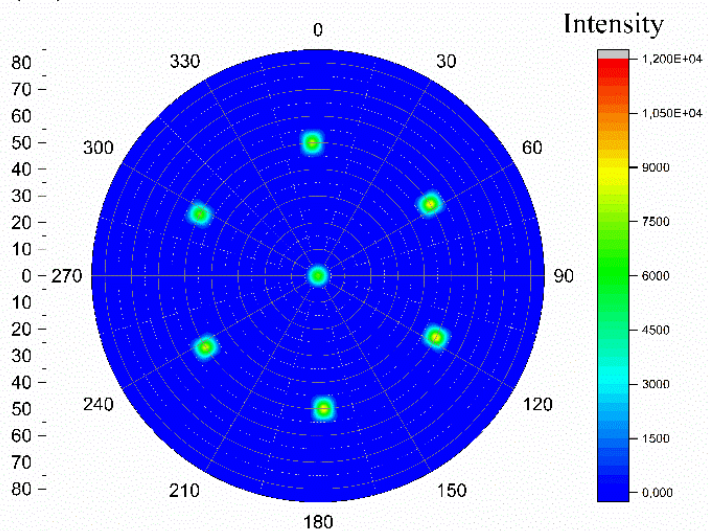

Figure 3.14: XRD of $\mathrm{GaN}$ deposited at $250{ }^{\circ} \mathrm{C}$ with $4 \mathrm{~s}$ of 4 and $9 \mathrm{~s} \mathrm{NH}_{3}$ plasma with a thickness of approximately $70 \mathrm{~nm}$. a) $\theta-2 \theta$ showing the (0002) and (0004) plane of hexagonal GaN b) GiXRD showing no present peaks indicating no tilted grains for $\mathrm{GaN}$ on SiC. c) Pole figure of (1013) plane for GaN showing six poles of

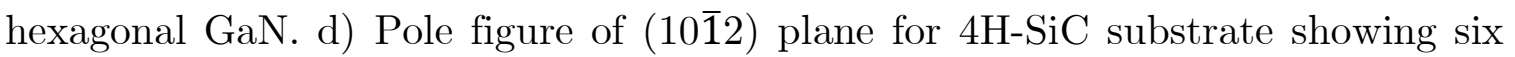
poles representing its six-fold symmetry.

Further structural characterization was undertaken using scanning transmission electron microscopy (STEM) with high angle annular dark field 
(HAADF) imaging and SAED). An overview cross-sectional STEM-HAADF image,

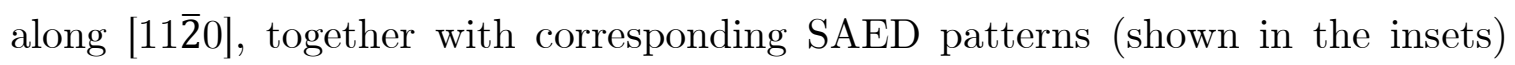
acquired from the film and substrate are shown in Figure 3.15a. According to the STEM-HAADF images recorded under strong elemental contrast (Z-contrast) conditions, two distinct regions in the film are observed. The film appears uniform starting from film/substrate interface for the first $\sim 6 \mathrm{~nm}$. Afterward, the growing film starts to develop columnar microstructure, leading to a pronounced surface roughening. The darker STEM-HAADF contrast dotted pattern in the columns and columnar boundaries can be attributed to the presents of point defects, e.g. vacancies and impurities, in the film.

The epitaxial grown GaN film on 4H-SiC can be identified by SAED pattern shown as insets in Figure 3.15a. The GaN is oriented with the c-axis perpendicular to the $4 \mathrm{H}-\mathrm{SiC}$ substrate surface, GaN [0002] || SiC [0002] and the in-plane relation

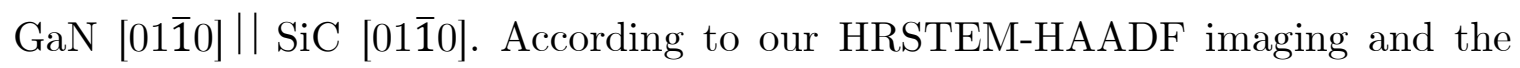
Fast Fourier Transforms (FFTs), the stacking of the GaN lattice planes is more ordered close to the GaN-SiC interface compared to the upper part of the film, Figure 3.15b. The arc-shaped diffraction spots associated with GaN (inset in Figure 3.15a), indicating part of GaN crystal is deviated from the ideal c-axis epitaxy, are ascribed to the curved stacking of (0002) planes for the upper part of the film, Figure $3.15 \mathrm{c}$. The lattice curvature together with various types of structural 
imperfections becomes more and more pronounced for the top part of the film. The GaN film can accommodate the stress in the beginning of the growth, the first $\sim 6$ $\mathrm{nm}$ as evidenced from Figure 3.15b. We believe that when the GaN grows over this critical thickness the increased stress (due to compressive lattice mismatch of $3 \%$ with the substrate) forces the GaN planes to curve. The curvature of the planes increases along the c-direction, further away from film/substrate interface. X-ray Rocking Curve (XRC) was employed on the epitaxial GaN, showing that the full width at half maximum (FWHM) for the (0002) plane was $523 \operatorname{arc} \sec \left(0.1452^{\circ}\right)$, Figure 3.17. This is on par with previous high temperature CVD study of GaN films where an AlN seed layer was deposited on the SiC substrate. ${ }^{112}$ These findings could revolutionize the transistor industry, especially for the production of high electron mobility transistors (HEMTs). Being able to deposit very thin GaN directly on $\mathrm{SiC}$ without the need for an AlN seed layer allows for increased design freedom when producing HEMTs, which could in turn improve their performance.

Finally, a thermal ALD route to GaN was investigated using 4 and $\mathrm{NH}_{3}$ without plasma discharge. The same pulse parameters were used as the plasma process, but a $16 \mathrm{~s}$ of $\mathrm{NH}_{3}$ pulse was used in a temperature range of $150-400{ }^{\circ} \mathrm{C}$. GaN films could be obtained also via this thermal process, however, the films were X-ray ( $\theta-2 \theta$ and GIXRD) amorphous. The XPS analysis showed the films contained high amounts of $\mathrm{C}$ and $\mathrm{O}$ impurities, 4 at.\% and 15 at.\%, respectively (Figure 
3.19). The Ga/N ratio could not be accurately be measured due to the Ga Auger overlap with $\mathrm{N}$ 1s. The high level of impurities could explain the amorphous nature of these films, which are likely to distort the long-range order of the atoms in the lattice. This also highlights the efficiency of the $\mathrm{NH}_{3}$ plasma to remove carbon and oxygen impurities from the growing film surface.

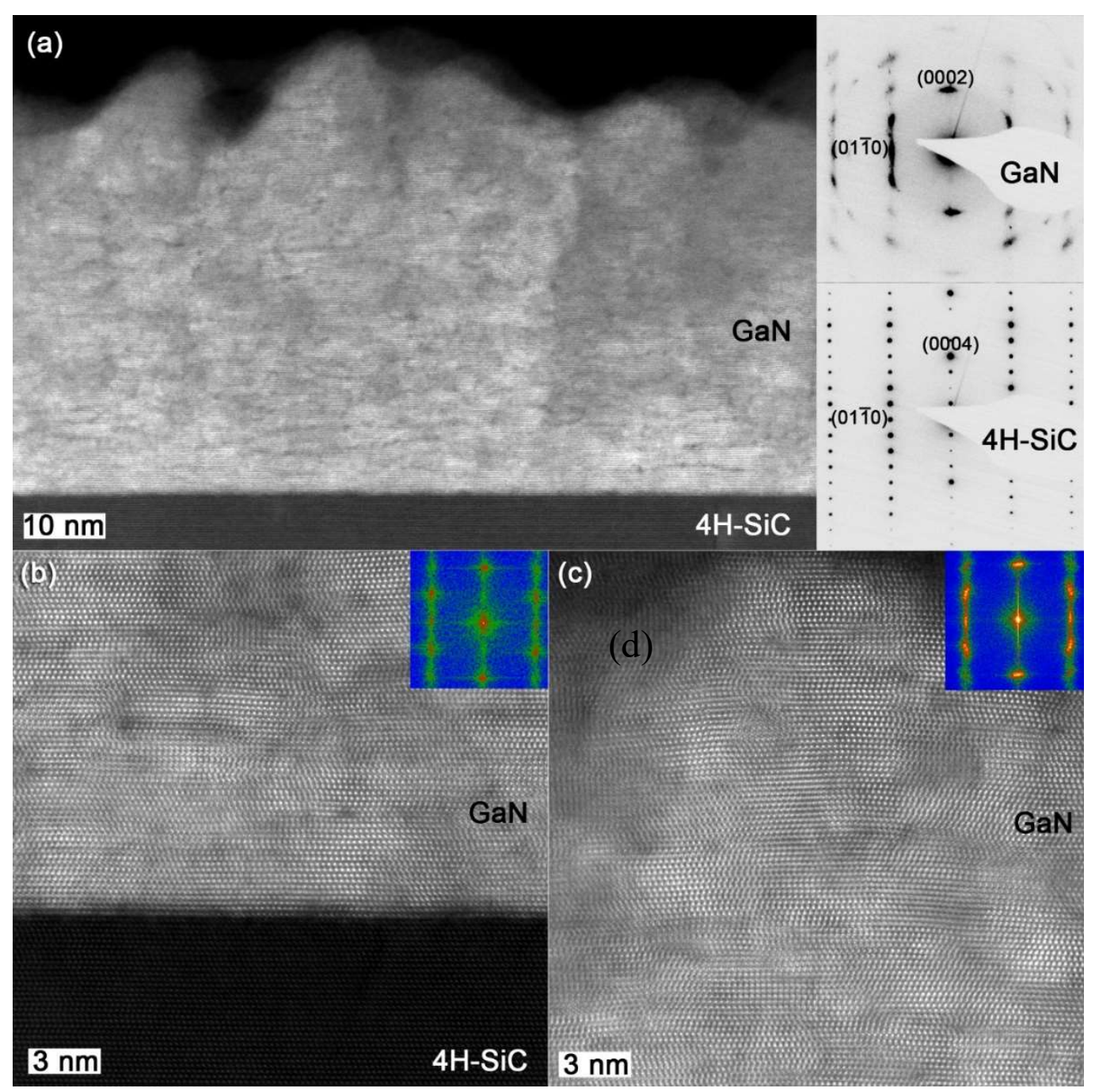

Figure 3.15: An overview STEM-HAADF image from the GaN film $\left(4+\mathrm{NH}_{3}\right.$ plasma) with a thickness of approximately $40 \mathrm{~nm}$ on $4 \mathrm{H}-\mathrm{SiC}$ together with SAED in the insets. HRSTEM-HAADF image from b) film-substrate interface and c) the top part of the film. FFT patterns are shown as insets in b) and c). 


\subsubsection{Summary and Conclusions}

We have successfully been able to deposit GaN on $\mathrm{Si}$ (100) and 4H-SiC (0001) using tris(dimethylamido)gallium(III) together with $\mathrm{NH}_{3}$ by both thermal and plasma ALD. The $\mathrm{NH}_{3}$ plasma process showed that surface saturation could be obtained with a GPC of $1.4 \AA$ /cycle after $4 \mathrm{~s}$ of 4 and $6 \mathrm{~s}$ of $\mathrm{NH}_{3}$ plasma. The growth per cycle was independent of temperature between $130-250{ }^{\circ} \mathrm{C}$ and the growth rate was 3-5 times higher than previously reported ALD of GaN. The deposited film at $250{ }^{\circ} \mathrm{C}$ showed a $\mathrm{Ga} / \mathrm{N}$ ratio of 0.97 with 2.8 at.\% of $\mathrm{C}$ and 3 at.\% of O measured by RBS/ERDA. A Tauc plot revealed that the deposited GaN had an optical bandgap of $3.42 \mathrm{eV}$. XPS measurements showed that the Fermi level lies closer to the conduction band in comparison to the valence band, indicating unintentionally n-type doped GaN films. Pole figure measurements showed that epitaxial GaN was deposited directly on $4 \mathrm{H}-\mathrm{SiC}$ (0001), without any buffer layer, with $\mathrm{NH}_{3}$ plasma at $250{ }^{\circ} \mathrm{C}$. The epitaxial relationships were determined as $\operatorname{GaN}(0002) \| \mathrm{SiC}(0004)$ and $\mathrm{GaN}(10 \overline{1} 3) \| \mathrm{SiC}(10 \overline{1} 2)$. SAED confirmed the epitaxial relationship between the GaN film and $\mathrm{SiC}$ substrate. This is to our knowledge the first epitaxial GaN on $\mathrm{SiC}$ without a buffer layer. The STEMHAADF images also revealed that the GaN planes start to curve along the growth direction after a critical thickness of approximately $6 \mathrm{~nm}$. XRC shows that the GaN (0002) has a FWHM of 523 arc sec which is similar to that of CVD grown 
GaN. The thermal route rendered X-ray ( $\theta-2 \theta$ and GIXRD) amorphous GaN in the temperature range of $150-400{ }^{\circ} \mathrm{C}$. The high impurity levels in the thermal GaN films are believed to be the reason for its amorphous nature.

These findings could open up the doors for new and smaller HEMTs based on group 13 nitrides. By being able to deposit high quality GaN films at these low temperatures also present the opportunity to deposit on temperature sensitive materials which has previously been hindered due to the high deposition temperatures. The ability to deposit GaN directly on $\mathrm{SiC}$ also reduces the number of material interfaces which could potentially improve the transistor performance.

\subsubsection{Acknowledgements}

This project was founded by the Swedish foundation for Strategic Research through the project "Time-resolved low temperature CVD for III-nitrides" (SSF-RMA 150018) and by the Knut and Alice Wallenberg foundation through the project "Bridging the THz gap" (no. KAW 2013.0049). Support from the Swedish research council VR-RFI (2017-00646_9) for the Accelerator based ion-technological center, and from the Swedish Foundation for Strategic Research (RIF14-0053) for the tandem accelerator laboratory in Uppsala is gratefully acknowledged. The authors acknowledge the Knut and Alice Wallenberg Foundation for supporting the 
Linköping electron microscopy laboratory and Seán T. Barry for access to TGA and DSC instruments at Carleton University, Canada.

\subsubsection{Supporting Information}

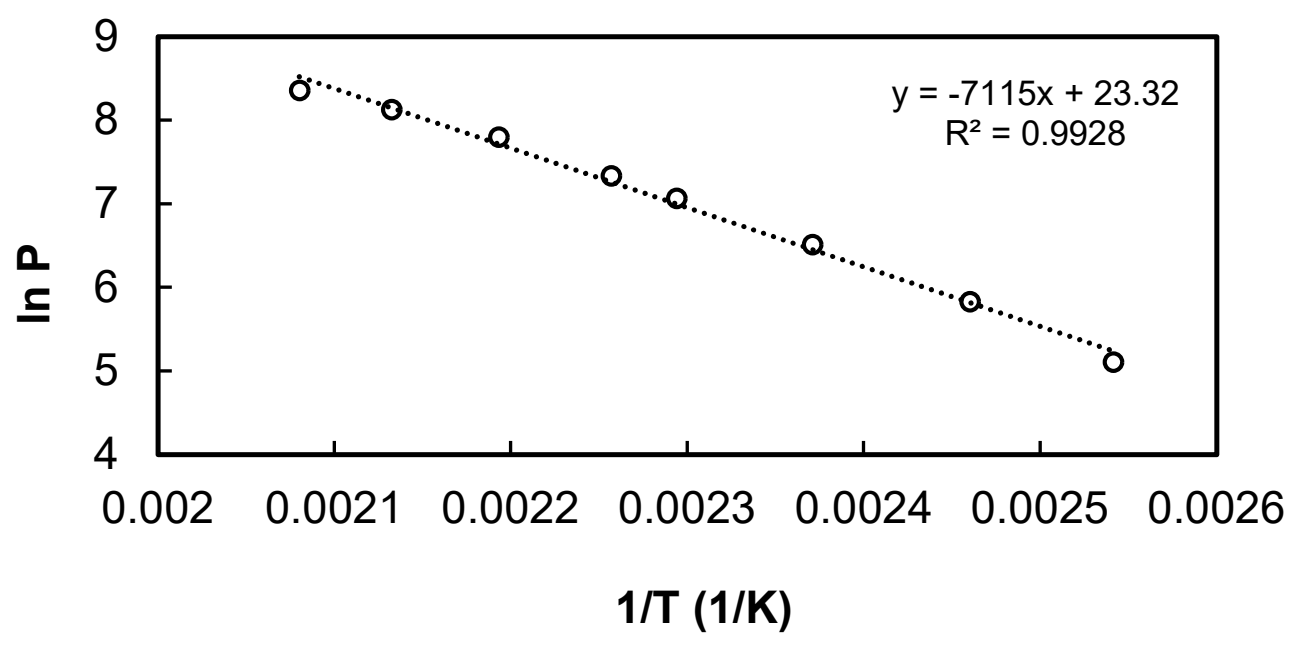

Figure 3.16: Plot of $\ln$ (vapour pressure) vs. $1 / \mathrm{T}$ for $\mathbf{4}$, obtained from TGA data and used to calculate the 1 Torr temperature.

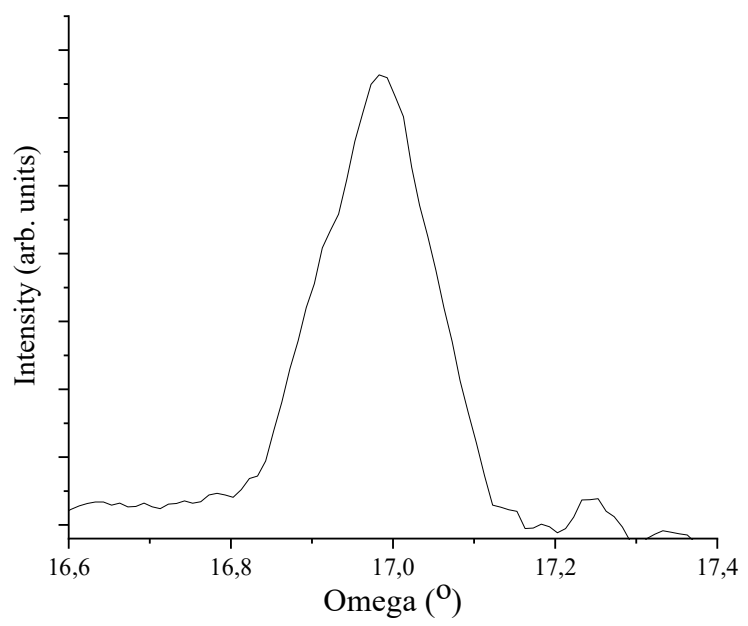

Figure 3.17: The XRC of the GaN (0002) plane showing a FWHM of 523 arc sec $\left(0.1452^{\circ}\right)$. Film deposited from 4 and $\mathrm{NH}_{3}$ plasma. 


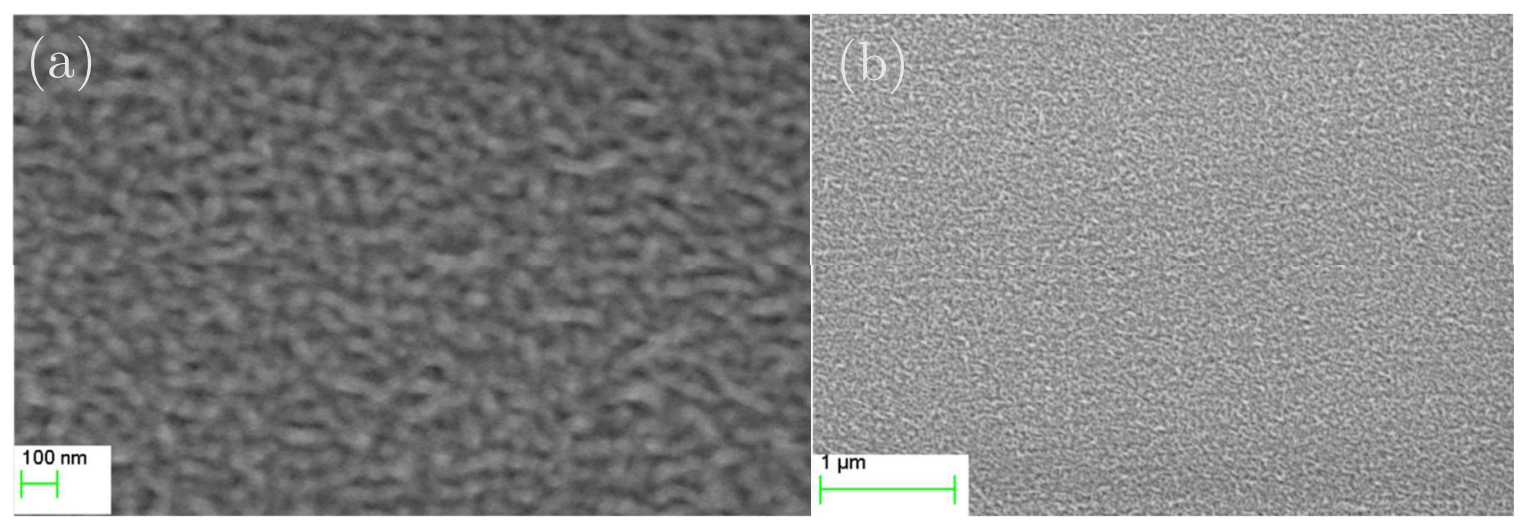

Figure 3.18: The top-view SEM of the GaN ( $4+\mathrm{NH}_{3}$ plasma) film on $\mathrm{SiC}$ with 4 $\mathrm{s}$ of 4 and $9 \mathrm{~s} \mathrm{NH}_{3}$ plasma deposited at $250{ }^{\circ} \mathrm{C}$ with both high magnification a) and low magnification b).

Ga $2 p$

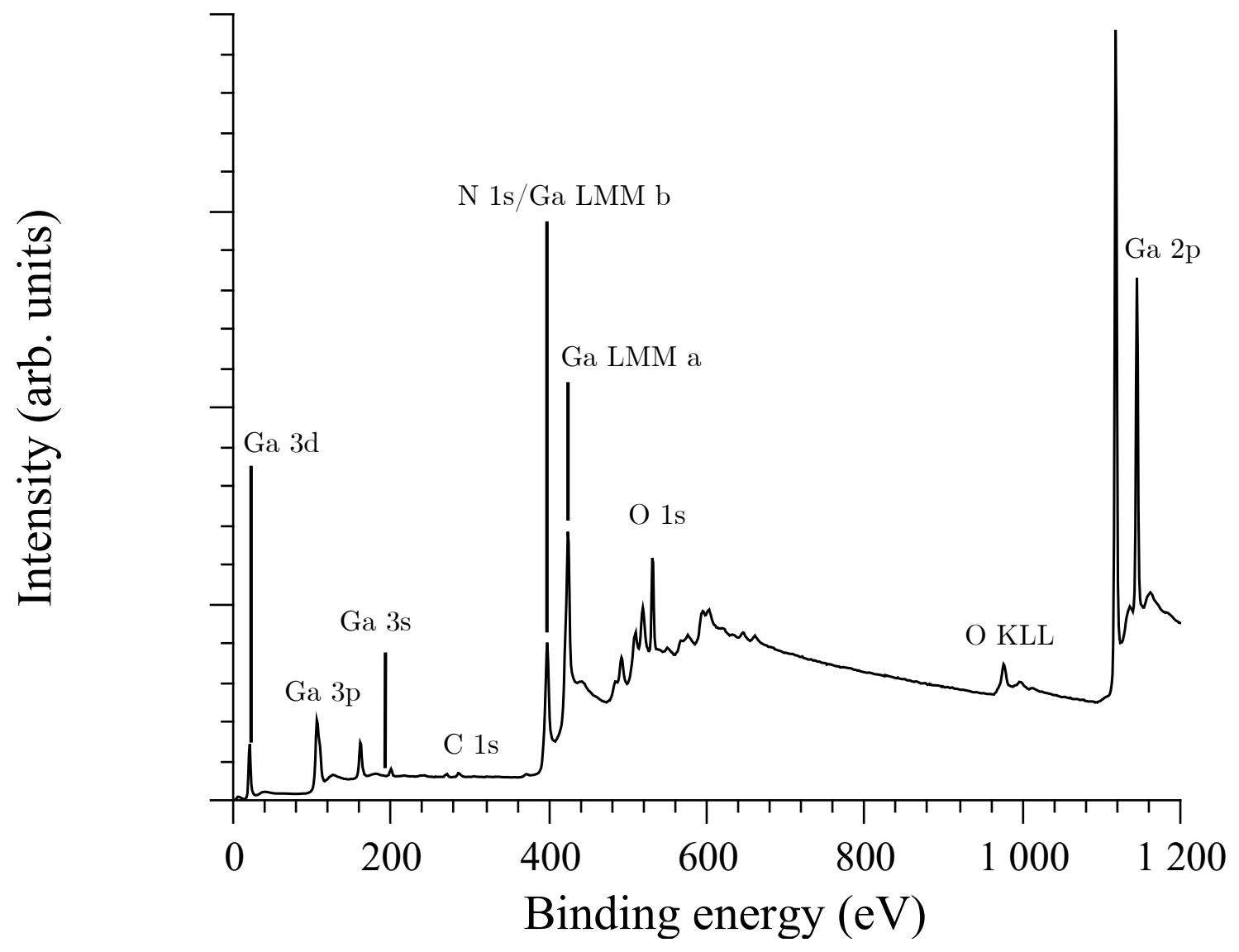

Figure 3.19: The XPS measurement with 4s pulse of 4 and $16 \mathrm{NH}_{3}$ pulse deposited at $200{ }^{\circ} \mathrm{C}$ after cleaning sputtering. 


\section{Chapter 4}

\section{Methylamines as Nitrogen Precursors in}

\section{Chemical Vapour Deposition of Gallium}

\section{Nitride}

Modified from the original manuscript published as:

Rönnby, K.; Buttera, S. C.; Rouf, P.; Barry, S. T.; Ojamäe, L.; Pedersen, H. Methylamines as Nitrogen Precursors in Chemical Vapor Deposition of Gallium

Nitride. J. Phys. Chem. C 2019, $123 \quad$ (11), 6701-6710. https://doi.org/10.1021/acs.jpcc.9b00482. 


\subsection{Abstract}

Chemical vapor deposition (CVD) is one of the most important techniques for depositing thin films of the group 13 nitrides (13-Ns), AlN, GaN, InN and their alloys, for electronic device applications. The standard CVD chemistry for 13-Ns use ammonia as the nitrogen precursor, however, this gives an inefficient CVD chemistry forcing N/13 ratios of 100/1 or more. Here we investigate the hypothesis that replacing the $\mathrm{N}-\mathrm{H}$ bonds in ammonia with weaker $\mathrm{N}-\mathrm{C}$ bonds in methylamines will permit better CVD chemistry, allowing lower CVD temperatures and an improved N/13 ratio. Quantum chemical computations shows that while the methylamines have a more reactive gas phase chemistry, ammonia has a more reactive surface chemistry. CVD experiments using methylamines failed to deposit a continuous film, while instead micrometer sized gallium droplets were deposited. This study shows that the nitrogen surface chemistry is most likely more important to consider than the gas phase chemistry when searching for better nitrogen precursors for 13-N CVD.

\subsection{Introduction}

The group 13 nitrides (13-Ns or III-Ns), AlN, GaN, InN and their alloys, form an increasingly important class of semiconductor materials with applications in light emitting diodes (LEDs), laser diodes, high electron mobility transistors (HEMTs), 
radio frequency (RF) devices, and power transistors. Regardless of the application, one of the most popular methods used to deposit thin layers of $13-\mathrm{Ns}$ is chemical vapor deposition (CVD), where typically trimethyl metal complexes $\left(\mathrm{M}\left(\mathrm{CH}_{3}\right)_{3}\right.$, where $\mathrm{M}=\mathrm{Al}$, Ga or $\mathrm{In})$ and ammonia $\left(\mathrm{NH}_{3}\right)$ are used as group 13 and nitrogen precursors, respectively. ${ }^{147}$ The reaction pathways of the trimethyl gallium $\left(\mathrm{Ga}\left(\mathrm{CH}_{3}\right)_{3}\right) / \mathrm{NH}_{3}$ system has been studied earlier, both experimentally and theoretically. ${ }^{148-153}$ The studies show a complex gas phase chemistry where both $\mathrm{Ga}\left(\mathrm{CH}_{3}\right)_{3}$ and $\mathrm{NH}_{3}$ could decompose to more reactive species that could deposit on the surface or react in the gas phase to form species containing both gallium and nitrogen. $\mathrm{Ga}\left(\mathrm{CH}_{3}\right)_{3}$ is a Lewis acid due to the empty p-orbital on the metal center and ammonia exists with a free electron pair on the nitrogen, making it a Lewis base; for this reason, Lewis adduct formation of $\mathrm{H}_{3} \mathrm{~N}: \mathrm{Ga}\left(\mathrm{CH}_{3}\right)_{3}$ is very likely, especially with the Le Chatelier's driving force provided by the large surplus of ammonia in the CVD gas mixture. It has been suggested that such adducts either break up at the high temperatures employed in CVD of 13-Ns or undergo a reaction where a M-N bond is formed with release of methane. ${ }^{154-156}$ The formation of $\mathrm{H}_{2} \mathrm{~N}$ $\mathrm{M}\left(\mathrm{CH}_{3}\right)_{2}$ could contribute to the growth of GaN by deposition, but might be also detrimental to the CVD process by leading to the formation of parasitic nitride nanoparticles in the gas phase. ${ }^{149,155,157-159}$ 
Despite the fact that CVD processes for 13 -Ns have reached a level of maturity that allows them to be used for industrial production of $13-\mathrm{N}$ based electronics, they typically require a very high $\mathrm{NH}_{3} / 13$ precursor ratio, typically 100-1000:1 for AlN and GaNError! Bookmark not defined, and up to $10^{5}: 1$ for InN, ${ }^{6}$ reflecting poorly tuned CVD chemistry. From a purely thermodynamic point of view, $\mathrm{NH}_{3}$ will mainly decompose under deposition conditions to highly stable dinitrogen and dihydrogen via the reactive species $\mathrm{NH}_{2}$ and $\mathrm{NH} .{ }^{160}$ However, kinetically, $\mathrm{NH}_{2}$ and $\mathrm{NH}$ intermediates only react to form dinitrogen and dihydrogen over longer timescales that are not typically accessible under normal CVD conditions. ${ }^{161}$ It has previously been demonstrated that $\mathrm{NH}_{3}$ has a very low reactivity at typical CVD temperatures for AlN and GaN depositions, and its decomposition by catalysis by iron from the CVD reactor's components has shown to improve ammonia reactivity. ${ }^{162}$ The need to use high $\mathrm{NH}_{3} / 13$ precursor ratios in CVD of group 13 nitrides is therefore the poor reactivity of $\mathrm{NH}_{3}$ to produce a more reactive species, rather than losing $\mathrm{NH}_{3}$ to the process by reacting to less active species. ${ }^{15}$

This study investigates whether replacing one or more of the $\mathrm{N}-\mathrm{H}$ bonds (bond dissociation energy: $450 \mathrm{~kJ} / \mathrm{mol}$ ) ${ }^{76}$ in $\mathrm{NH}_{3}$ with $\mathrm{N}-\mathrm{C}$ bonds (bond dissociation energy $356 \mathrm{~kJ} / \mathrm{mol})^{76}$ will lead to a more efficient nitrogen precursor; this change would produce a precursor with weaker bonds and lower symmetry as a result of 
substituting one or two hydrogens with methyl groups, hopefully increasing its reactivity to deposit group 13 nitride films. Quantum chemical calculations were used to study the possible gas phase chemical reactions in the decomposition of $\mathrm{NH}_{3-\mathrm{x}}\left(\mathrm{CH}_{3}\right)_{\mathrm{x}}$ with $\mathrm{x}=0-3$. The subsequent surface chemical interactions with the reactive fragments with a GaN surface were also studied using quantum chemical calculations. Since the Lewis adduct formation between acidic group 13 precursors and basic $\mathrm{N}$ precursors is an important aspect of the gas phase chemistry for CVD of $13-\mathrm{Ns}$, it has also been studied by quantum chemical computations in this work. The quantum chemical study is further compared to an experimental CVD study where $\mathrm{NH}_{2} \mathrm{CH}_{3}, \quad \mathrm{NH}\left(\mathrm{CH}_{3}\right)_{2}$ and $\mathrm{N}\left(\mathrm{CH}_{3}\right)_{3}$ were compared to $\mathrm{NH}_{3}$ as nitrogen precursors in a CVD process with $\mathrm{Ga}\left(\mathrm{CH}_{3}\right)_{3}$.

\subsection{Experimental}

\section{Computational Details}

Quantum chemical calculations were carried out with the Gaussian09 software suite. ${ }^{82}$ The high accuracy Gaussian 4 (G4) ${ }^{163}$ composite method was used for calculations on the gas phase molecular species, which enabled the derivation of thermochemical data for decomposition and adducts reactions. While the G4 method is well established for lighter elements ${ }^{164}$ there is less experience with its performance for e.g. gallium. Additional calculations using the coupled cluster 
approach $^{165}$ were therefore performed for comparison. The G4 method was found to somewhat overestimate the $\mathrm{Ga}-\mathrm{CH}_{3}$ bond energy (around $10 \mathrm{~kJ} / \mathrm{mol}$ ) compared to those calculated at $\operatorname{CCSD}(\mathrm{T}) /$ aug-cc-pVTZ level of theory. The calculated adduct formation energies using G4 level of theory, $74.51 \mathrm{~kJ} / \mathrm{mol}$ for the enthalpy of forming the adduct between $\mathrm{NH}_{3}$ and $\mathrm{Ga}\left(\mathrm{CH}_{3}\right)_{3}$, are in good agreement with experimental values, -68.20 to $-77.82 \mathrm{~kJ} / \mathrm{mol}$ for the same reaction. ${ }^{166,167}$ The hybrid density functional theory (DFT) B3LYP method with the D3 version of Grimme's empirical dispersion ${ }^{168}$ and the $6-31 \mathrm{G}(2 \mathrm{df}, \mathrm{p})$ was used as the basis set for the surface interaction studies. For the surface studies, a hydrogen terminated model (0001) surface in the form of a $(\mathrm{GaN}){ }_{13} \mathrm{H}_{32}$ cluster was used (See Fig. $4.8 \mathrm{in}$ the supporting information). The effect of the size of the cluster on adsorption energies was investigated and are summarized in the supporting information. It was found that the effect was small, i.e. enlarging the cluster changed the adsorption energy by less than $1.84 \mathrm{~kJ} / \mathrm{mol}$.

The geometries, for both equilibrium and transition state structures were optimized using the Berny algorithm as implemented in the Gaussian program with standard convergence criteria. The equilibrium structures were confirmed to have zero imaginary vibrational modes by vibrational normal-mode calculations while the transition state structures were confirmed to have one imaginary mode connecting the reactant and product structures. Gibbs free energies were obtained 
by adding zero-point energies and temperature-dependent energy contributions to the electronic energies along with entropic terms as computed from the vibrational normal-mode frequencies and the optimized geometries. ${ }^{169}$ An investigation of the error of treating the internal rotations as vibration was performed; see supporting information for details. Due to the small difference in energy (less than $2 \mathrm{~kJ} / \mathrm{mol}$ for enthalpy and $3.2 \mathrm{~kJ} / \mathrm{mol}$ for free energy) for the two methods, we chose to not include correction for the internal rotations in our presented calculations.

\section{Experimental Details}

The depositions of GaN films were undertaken in a horizontal hot-wall CVD system $^{170}$ operating at 50 mbar with a carrier gas mixture of palladium membrane purified hydrogen $\left(\mathrm{H}_{2}\right)$ and nitrogen $\left(\mathrm{N}_{2}\right)(99.999 \%)$, at a volumetric $\mathrm{H}_{2} / \mathrm{N}_{2}$ ratio of 6.33. Films were deposited on nominally on-axis $4 \mathrm{H}-\mathrm{SiC}$ (0001) substrates, cut into $2 \times 2 \mathrm{~cm}^{2}$ pieces, washed using standard RCA cleaning solutions: ${ }^{127}$ they were submerged in $\mathrm{NH}_{3}: \mathrm{H}_{2} \mathrm{O}_{2}: \mathrm{H}_{2} \mathrm{O}$ 1:1:5, rinsed in deionized water, submerged in $\mathrm{HCl}: \mathrm{H}_{2} \mathrm{O}_{2}: \mathrm{H}_{2} \mathrm{O}$ 1:1:6, rinsed again in deionized water, and finally dried with dry $\mathrm{N}_{2}$. Before each CVD experiment, an epitaxial aluminum nitride (AlN) nucleation layer was grown on the $\mathrm{SiC}$ substrate using $\mathrm{Al}\left(\mathrm{CH}_{3}\right)_{3}$ (SAFC Hightec EpiPure grade) and $\mathrm{NH}_{3}(99.9999 \%$, further purified using a Nanochem purifier down to $<$

$1 \mathrm{ppb}$ for important contaminants such as water and oxygen) with a flow ratio of $\mathrm{NH}_{3} / \mathrm{Al}\left(\mathrm{CH}_{3}\right)_{3}=1043$ at $1200{ }^{\circ} \mathrm{C}$. The temperature was then lowered to $800-1000$ 
${ }^{\circ} \mathrm{C}$ for the growth of GaN directly on the AlN layer using $\mathrm{Ga}\left(\mathrm{CH}_{3}\right)_{3}$ (SAFC Hightec EpiPure grade) and $\mathrm{NH}_{3}, \mathrm{NH}_{2} \mathrm{CH}_{3}(\mathrm{AGA}, 99 \%), \mathrm{NH}\left(\mathrm{CH}_{3}\right)_{2}(\mathrm{AGA}, 99 \%)$ or $\mathrm{N}\left(\mathrm{CH}_{3}\right)_{3}$ (AGA, $99 \%$ ). All methylamines where further purified by a Micro Torr gas filter to reduce water and oxygen to less than $1 \mathrm{ppb}$.

We note, and would like to point out, that the methylamines, as compared to ammonia, are more challenging to work with in CVD experiments. The lower vapor pressure of the methylamines led, at one point, to condensation of amine in the gas lines and we therefore recommend that the gas lines and mass flow controllers are heated when attempting to replace ammonia with methylamines in a CVD setup.

The morphology of the grown films was characterized using an optical microscope with Nomarski differential interference contrast and by a LEO 1550 Gemini scanning electron microscope (SEM) equipped with a field emission gun using 3-20 kV acceleration. Energy-dispersive X-ray spectroscopy (EDS) was used in the SEM for elemental analysis. An interference method ${ }^{171}$ was used to determine the thickness of the epitaxial layers. XRD measurements were performed by employing PANalytical EMPYREAN MRD X-Ray diffractometer equipped with a Cu-anode x-ray tube and 5-axis (x-y-z-v-u) sample stage. For the determination of the crystalline structure of the thin films, glancing incidence XRD (GI-XRD) was conducted. For these measurements, capillary optics on the incident beam side and 
parallel plate collimator on the detector side were used. The $\mathrm{Cu} \mathrm{K} \beta$ line was removed by a Ni filter.

\subsection{Results and Discussion}

\section{Gas Phase Decomposition of $\mathrm{NH}_{3-\mathrm{x}}\left(\mathrm{CH}_{3}\right)_{\mathrm{x}}$}

The optimized geometries of the $\mathrm{NH}_{3-\mathrm{x}}\left(\mathrm{CH}_{3}\right) \mathrm{x}$ species and their decomposition products are given in Fig. 4.1. The studied gas phase decomposition reactions are summarized in Scheme 4.1. In these reaction paths the nitrogen atom consecutively loses a ligand (hydrogen atom or methyl group) and forms more reactive nitrogen radical species. The reactions are labeled "H" and "C" and correspond to the release of a hydrogen (vertical transitions) or a methyl radical (diagonal transitions), respectively. The "RE" reaction is a rearrangement of $\mathrm{NCH}_{3}$ to methanimine $\left(\mathrm{NHCH}_{2}\right)$.

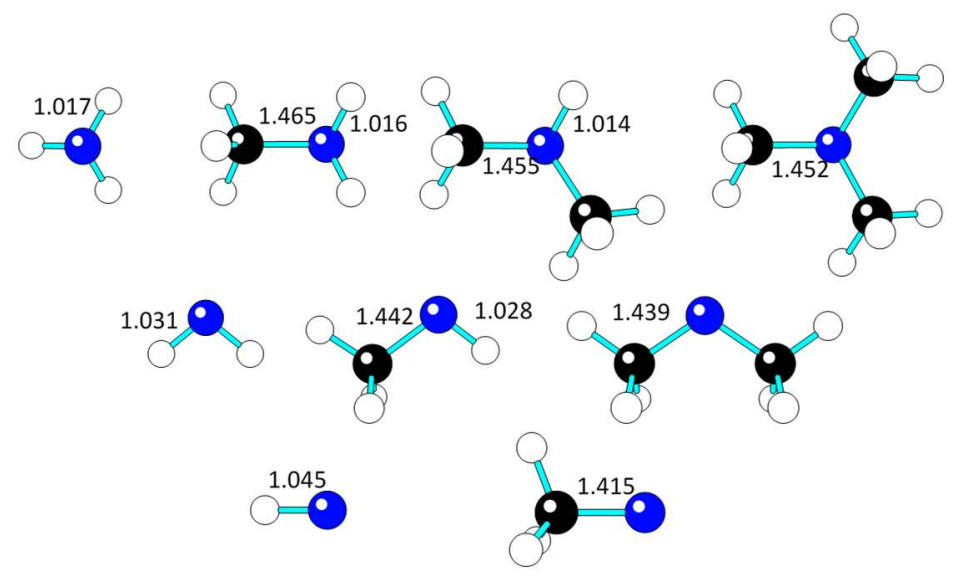

Figure 4.1: Optimized geometries and bond lengths in Ångström for $\mathrm{NH}_{\mathrm{x}}\left(\mathrm{CH}_{3}\right)_{\mathrm{n}-\mathrm{x}}$ $(\mathrm{n}=3,2,1)$ Blue atoms are nitrogen, black are carbon and white are hydrogen. 


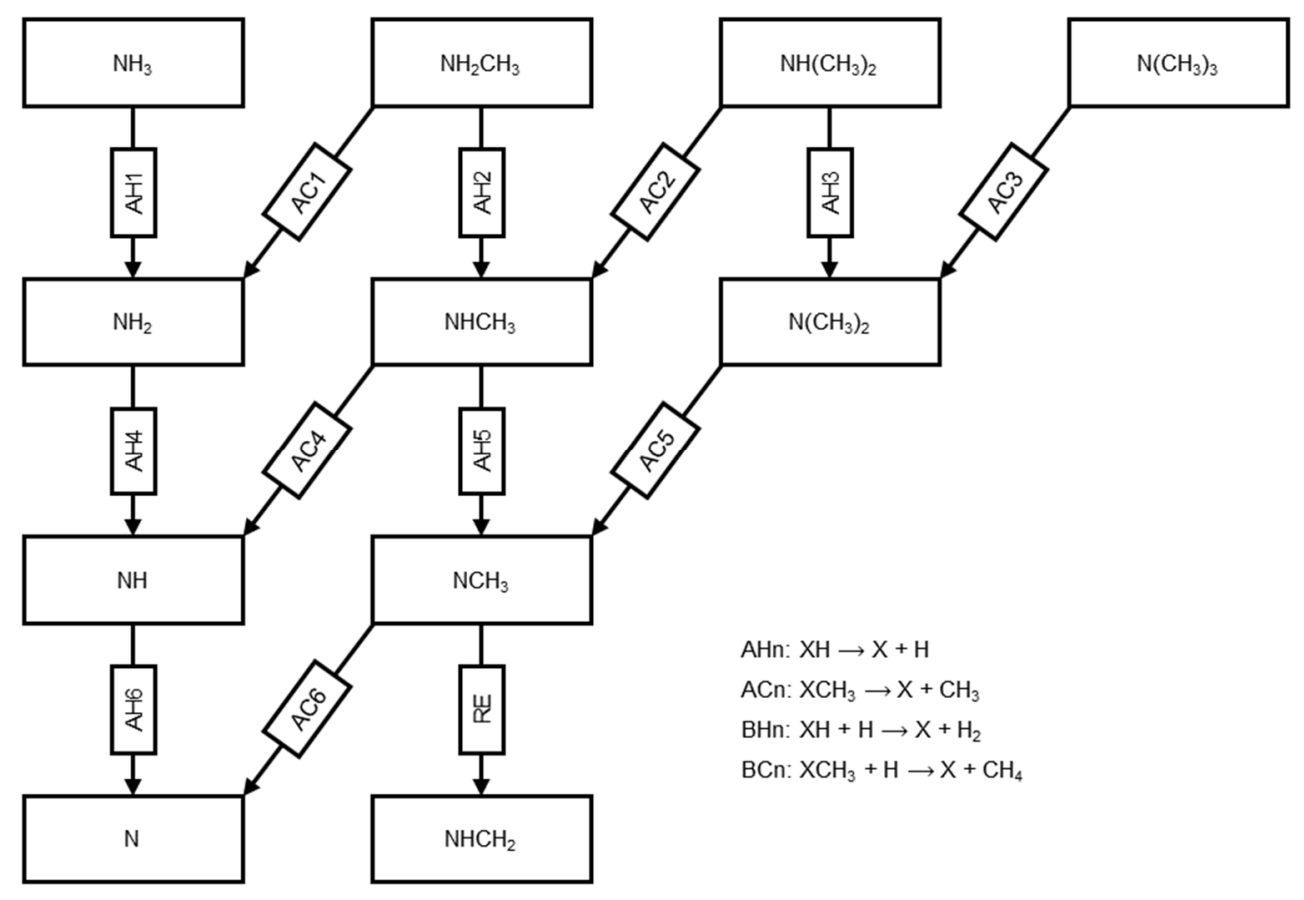

Scheme 4.1: Decomposition pathways for the unimolecular decomposition of methyl amines, $\mathrm{NH}_{3-\mathrm{x}}\left(\mathrm{CH}_{3}\right)_{\mathrm{x}}$ with $\mathrm{x}=0-3$. Reactions labeled "AHn" release a hydrogen radical while reactions labeled "ACn" release a methyl radical. Reaction "RE" is a rearrangement.

The energies of the unimolecular decompositions of ammonia and the three methylamines are presented Table 4.1 and graphically in Figs. 4.10-4.11. All reactions are endoenergetic $\left(\Delta_{\mathrm{r}} \mathrm{E}^{\text {elec }}>0\right)$ and endothermic $\left(\Delta_{\mathrm{r}} \mathrm{H}^{\circ}>0\right)$. All reactions except methyl elimination from $\mathrm{N}\left(\mathrm{CH}_{3}\right)_{3}(\mathrm{AC} 3)$ are also endergonic $\left(\Delta_{\mathrm{r}} \mathrm{G}^{\circ}>0\right)$, making the reactants more favorable than the decomposition products. The free energies are lower at elevated temperatures due to an increase in entropy, making the difference between $\Delta_{\mathrm{r}} \mathrm{H}^{\circ}$ and $\Delta_{\mathrm{r}} \mathrm{G}^{\circ}$ larger at higher temperatures. In general, the reaction free energy is lower for a loss of methyl than for the loss of a hydrogen radical and the energy difference increases at higher temperatures, which is in line 
with the bond energies. The triplet spin state of $\mathrm{NCH}_{3}$ has a lower energy than the singlet state, but the molecule can undergo rearrangement (RE) to the much more stable methanimine $\left(\mathrm{NHCH}_{2}\right)$ by migration of a hydrogen from the methyl to the nitrogen, thereby forming a nitrogen-carbon double bond. The structures of these molecules are given in Fig. 4.12 in the supporting information. The calculated N$\mathrm{C}$ bond length is consistent with the formation of a double bond. This rearrangement was exothermic and had a negative reaction free energy at both STP and CVD conditions at all temperatures. The temperature dependence of the free energy is very small due to the entropy change being small for an internal rearrangement.

In addition to unimolecular decomposition, there also exists a possibility for ammonia and methylamines to decompose via hydrogen radical assisted abstraction. Hydrogen radicals are abundant under typical CVD conditions given the high temperatures and carrier gas mixture used. ${ }^{172}$ The bimolecular decomposition reactions also follow Scheme 1 with the difference that a hydrogen radical is included as a co-reactant and reacts to form molecular dihydrogen or methane as co-products. The energies of the bimolecular decompositions of ammonia and the three methylamines are given in Table 4.2 and graphically in Figs. 4.10-4.11. All reactions except the abstraction of a hydrogen from ammonia are exothermic and exergonic $\left(\Delta_{\mathrm{r}} \mathrm{G}^{\circ}<0\right)$ at all investigated CVD temperatures. 
Table 4.1: Reaction enthalpies and free energies for unimolecular decomposition of ammonia and methylamines at CVD conditions: $300-1300{ }^{\circ} \mathrm{C}$ and total pressure 50 mbar. Energies given in $\mathrm{kJ} / \mathrm{mol}$. Reaction indices refer to Scheme 4.1.

\begin{tabular}{|c|c|c|c|c|c|c|}
\hline \multirow{2}{*}{$\begin{array}{c}\text { Reaction } \\
\text { index }\end{array}$} & \multicolumn{6}{|c|}{$\Delta_{\mathrm{r}} \mathrm{H}^{\circ}$} \\
\hline & $300{ }^{\circ} \mathrm{C}$ & $500{ }^{\circ} \mathrm{C}$ & $700{ }^{\circ} \mathrm{C}$ & $900{ }^{\circ} \mathrm{C}$ & $1100{ }^{\circ} \mathrm{C}$ & $1300{ }^{\circ} \mathrm{C}$ \\
\hline AH1 & 451.2 & 453.7 & 455.6 & 457.1 & 458.1 & 458.9 \\
\hline AH2 & 419.1 & 421.6 & 423.5 & 424.9 & 426.0 & 426.7 \\
\hline AH3 & 395.0 & 397.6 & 399.5 & 400.9 & 401.9 & 402.6 \\
\hline AH4 & 391.8 & 394.4 & 396.5 & 398.3 & 399.9 & 401.1 \\
\hline AH5 & 357.1 & 358.8 & 360.1 & 361.1 & 361.7 & 362.1 \\
\hline AH6 & 338.4 & 340.8 & 343.0 & 345.1 & 347.0 & 348.7 \\
\hline AC1 & 354.5 & 355.0 & 354.2 & 352.6 & 350.6 & 348.2 \\
\hline AC2 & 340.5 & 340.7 & 339.7 & 338.0 & 335.8 & 333.3 \\
\hline AC3 & 329.2 & 329.2 & 328.1 & 326.3 & 324.1 & 321.5 \\
\hline AC4 & 327.2 & 327.8 & 327.2 & 326.1 & 324.5 & 322.6 \\
\hline AC5 & 302.6 & 302.0 & 300.4 & 298.2 & 295.7 & 292.9 \\
\hline AC6 & 308.5 & 309.8 & 310.2 & 310.1 & 309.7 & 309.2 \\
\hline \multirow[t]{2}{*}{ RE } & -226.0 & -226.6 & -227.2 & -227.8 & -228.3 & -228.7 \\
\hline & \multicolumn{6}{|c|}{$\Delta_{\mathrm{r}} \mathrm{G}^{\circ}$} \\
\hline AH1 & 368.7 & 339.4 & 309.6 & 279.5 & 249.1 & 218.6 \\
\hline AH2 & 330.1 & 298.6 & 266.5 & 234.1 & 201.5 & 168.7 \\
\hline AH3 & 299.8 & 266.2 & 232.0 & 197.4 & 162.6 & 127.7 \\
\hline AH4 & 313.9 & 286.3 & 258.0 & 229.4 & 200.5 & 171.3 \\
\hline AH5 & 276.0 & 247.5 & 218.5 & 189.3 & 159.9 & 130.5 \\
\hline AH6 & 269.7 & 245.3 & 220.3 & 194.9 & 169.2 & 143.1 \\
\hline $\mathrm{AC} 1$ & 242.9 & 203.8 & 164.8 & 126.1 & 87.6 & 49.4 \\
\hline $\mathrm{AC2}$ & 219.7 & 177.4 & 135.3 & 93.4 & 51.9 & 10.7 \\
\hline AC3 & 199.9 & 154.7 & 109.7 & 65.0 & 20.6 & -23.4 \\
\hline $\mathrm{AC} 4$ & 226.7 & 191.5 & 156.3 & 121.3 & 86.6 & 52.0 \\
\hline AC5 & 195.9 & 158.7 & 121.8 & 85.3 & 49.2 & 13.5 \\
\hline AC6 & 220.4 & 189.4 & 158.2 & 127.0 & 95.8 & 64.7 \\
\hline $\mathrm{RE}$ & -223.4 & -222.4 & -221.2 & -220.0 & -218.6 & -217.1 \\
\hline
\end{tabular}


As in the unimolecular case, the effect of increased temperature lowers the reaction enthalpy and free energy. Temperature effects are smaller than for the unimolecular decomposition due to the smaller change in entropy, since the number of reactants and products are the same. The reaction energy for the removal of a ligand is, as in the unimolecular case, lower for the methyl than the hydrogen.

The mechanism for hydrogen abstraction was assumed to be that initially a hydrogen atom can approach either a hydrogen bonded to the nitrogen or a methyl ligand. As the hydrogen approaches the ligand the bond to the nitrogen is elongated and the ligand can be released in the form of a dihydrogen or methane molecule. Transition states for the mechanism were found, and the corresponding activation energies were computed for the first bimolecular decomposition; the enthalpy and free energy of the transition states relative to the reactants are listed in Table 4.3 and graphically in Figs. 4.10-4.11. The activation enthalpies for all transition states show very little variance within the temperature range used for CVD, while the activation free energy increases with increased temperature. Noteworthy is that the free energy barrier of hydrogen elimination depends strongly on the number of methyl ligands, while the barrier for the elimination of methane is fairly constant for all the methylamines. The barrier for hydrogen elimination is also lower than the barrier for methane elimination, making the hydrogen elimination the preferred 
kinetic path, while the reaction free energy makes the methane elimination the preferred thermodynamic path.

\section{Adduct Formation Between $\mathrm{NH}_{3-\mathrm{x}}\left(\mathrm{CH}_{3}\right)_{\mathrm{x}}$ and $\mathrm{Ga}\left(\mathrm{CH}_{3}\right)_{3}$}

The adduct formation between $\mathrm{Ga}\left(\mathrm{CH}_{3}\right)_{3}$ and the methylamines with subsequent alkane release reactions are summarized in Scheme 4.2. The reaction energies are given in Table 4.4 and graphically in Figs. 4.10-4.11. The adduct formation reactions (CAn) are all exothermic and exergonic at STP but endergonic for all reactions at CVD temperatures and pressures. The reaction free energy increases as the temperature is increased due to the decrease in entropy from the formed adducts. The reaction free energy for the three first reactions (CA1-3) decreases with increased number of methyl ligands on the nitrogen, possibly due to a stabilizing effect of the positive charge at the nitrogen by the methyl groups. For the last adduct reaction (CA4), the reaction free energy increases, possibly as an effect of steric factors. The optimized geometries for the adducts are given in Fig. 4.13 in the supporting information.

The removal of ligands from the adducts is categorized in two groups: the release of methane (CMn) and release of ethane (CEn). At STP and lower CVD temperatures, the removal of both methane and ethane are endothermic and at higher temperatures they are exothermic, except for CE1 which is exothermic at all temperatures. The temperature at which the enthalpy changes sign increases 
Table 4.2: Reaction enthalpies and free energies for bimolecular decomposition of ammonia and methylamines at CVD conditions of $300-1300^{\circ} \mathrm{C}$ and 50 mbar. Bimolecular decomposition reactions follow Scheme 4.1 with the difference that a hydrogen radical is included as a co-reactant and reacts to form molecular hydrogen or methane as a co-product. All energies are given in $\mathrm{kJ} / \mathrm{mol}$.

\begin{tabular}{|c|c|c|c|c|c|c|}
\hline \multirow{2}{*}{$\begin{array}{c}\text { Reaction } \\
\text { index }\end{array}$} & \multicolumn{6}{|c|}{$\Delta_{\mathrm{r}} \mathrm{H}^{\circ}$} \\
\hline & $300{ }^{\circ} \mathrm{C}$ & $500{ }^{\circ} \mathrm{C}$ & $700{ }^{\circ} \mathrm{C}$ & $900{ }^{\circ} \mathrm{C}$ & $1100^{\circ} \mathrm{C}$ & $1300^{\circ} \mathrm{C}$ \\
\hline BH1 & 10.4 & 10.4 & $\begin{array}{l}9.9 \\
\end{array}$ & 9.0 & 7.9 & 6.6 \\
\hline BH2 & -21.7 & -21.7 & -22.2 & -23.1 & -24.3 & -25.6 \\
\hline BH3 & -45.8 & -45.7 & -46.3 & -47.2 & -48.4 & -49.8 \\
\hline BH4 & -49.0 & -48.9 & -49.2 & -49.7 & -50.4 & -51.2 \\
\hline BH5 & -83.7 & -84.5 & -85.6 & -87.0 & -88.5 & -90.2 \\
\hline BH6 & -102.4 & -102.5 & -102.7 & -103.0 & -103.3 & -103.6 \\
\hline $\mathrm{BC} 1$ & -88.8 & -91.4 & -94.4 & -97.4 & -100.5 & -103.4 \\
\hline BC2 & -102.8 & -105.7 & -108.8 & -112.0 & -115.2 & -118.3 \\
\hline BC3 & -114.1 & -117.2 & -120.4 & -123.7 & -126.9 & -130.1 \\
\hline BC4 & -116.1 & -118.6 & -121.3 & -124.0 & -126.6 & -129.0 \\
\hline BC5 & -140.7 & -144.4 & -148.2 & -151.8 & -155.3 & -158.7 \\
\hline \multirow[t]{2}{*}{ BC6 } & -134.8 & -136.6 & -138.4 & -140.0 & -141.3 & -142.4 \\
\hline & \multicolumn{6}{|c|}{$\Delta_{\mathrm{r}} \mathrm{G}^{\circ}$} \\
\hline BH1 & 3.6 & 1.1 & -1.2 & -3.4 & -5.4 & $\begin{array}{l}-7.3 \\
\end{array}$ \\
\hline BH2 & -35.0 & -39.7 & -44.3 & -48.8 & -53.0 & -57.1 \\
\hline BH3 & -65.3 & -72.1 & -78.9 & -85.5 & -91.9 & -98.2 \\
\hline BH4 & -51.2 & -52.0 & -52.8 & -53.5 & -54.1 & -54.5 \\
\hline BH5 & -89.1 & -90.8 & -92.3 & -93.6 & -94.6 & -95.4 \\
\hline BH6 & -95.4 & -93.0 & -90.5 & -87.9 & -85.4 & -82.7 \\
\hline BC1 & -101.5 & -105.5 & -108.8 & -111.4 & -113.6 & -115.3 \\
\hline BC2 & -124.7 & -131.9 & -138.3 & -144.1 & -149.3 & -154.0 \\
\hline BC3 & -144.4 & -154.6 & -163.9 & -172.5 & -180.5 & -188.1 \\
\hline BC4 & -117.6 & -117.8 & -117.2 & -116.1 & -114.6 & -112.7 \\
\hline BC5 & -148.5 & -150.6 & -151.8 & -152.2 & -151.9 & -151.2 \\
\hline BC6 & -124.0 & -119.9 & -115.4 & -110.5 & -105.4 & -100.0 \\
\hline
\end{tabular}


Table 4.3: Activation enthalpies and free energies for bimolecular decomposition, listed in Table 4.2 , at CVD conditions $\left(300-1300^{\circ} \mathrm{C}\right.$ and $\left.50 \mathrm{mbar}\right)$. All energies are given in $\mathrm{kJ} / \mathrm{mol}$.

\begin{tabular}{|c|c|c|c|c|c|c|}
\hline \multirow{2}{*}{$\begin{array}{c}\text { Reaction } \\
\text { index }\end{array}$} & \multicolumn{6}{|c|}{$\Delta_{\mathrm{r}} \mathrm{H}^{\circ}$} \\
\hline & $300{ }^{\circ} \mathrm{C}$ & $500{ }^{\circ} \mathrm{C}$ & $700{ }^{\circ} \mathrm{C}$ & $900{ }^{\circ} \mathrm{C}$ & $1100{ }^{\circ} \mathrm{C}$ & $1300{ }^{\circ} \mathrm{C}$ \\
\hline$\overline{\mathrm{BH} 1}$ & 52.8 & 52.2 & 51.9 & 51.8 & 51.7 & 51.5 \\
\hline BH2 & 34.6 & 34.1 & 34.0 & 33.9 & 33.8 & 33.6 \\
\hline BH3 & 20.0 & 19.7 & 19.6 & 19.6 & 19.4 & 19.3 \\
\hline BC1 & 132.9 & 132.3 & 131.7 & 131.0 & 130.2 & 129.5 \\
\hline BC2 & 131.7 & 131.1 & 130.4 & 129.7 & 128.9 & 128.1 \\
\hline \multirow[t]{2}{*}{ BC3 } & 127.6 & 127.0 & 126.3 & 125.6 & 124.8 & 124.0 \\
\hline & \multicolumn{6}{|c|}{$\Delta_{\mathrm{r}} \mathrm{G}^{\circ}$} \\
\hline BH1 & 124.9 & 150.2 & 175.6 & 201.0 & 226.4 & 251.9 \\
\hline BH2 & 106.5 & 131.6 & 156.9 & 182.2 & 207.4 & 232.7 \\
\hline BH3 & 91.3 & 116.3 & 141.3 & 166.3 & 191.3 & 216.3 \\
\hline BC1 & 204.4 & 229.4 & 254.6 & 280.0 & 305.4 & 331.0 \\
\hline BC2 & 203.2 & 228.3 & 253.5 & 278.8 & 304.3 & 329.9 \\
\hline BC3 & 198.8 & 223.7 & 248.8 & 274.0 & 299.4 & 324.9 \\
\hline
\end{tabular}

with increasing number of methyl ligands on the nitrogen atom. The reaction free energy is negative for all the removal reactions, and due to the increase in entropy, the reaction is more favorable at higher temperatures. As with the reaction entropy, the reaction free energy is lower for the removal of ethane than for the removal of methane for the mono- and dimethylamine adducts. The sum reaction of adduct formation plus the release of methane or ethane are exothermic at all calculated temperatures; at STP and lower CVD temperatures, the reaction free energy is 
negative. Although the release reactions are entropically favorable, the total reaction $(\mathrm{CAx}+\mathrm{CMy} / \mathrm{CEy})$ has a decrease in entropy. This indicates that the entropy loss in the formation of the adducts is larger than the entropy gain from release of an alkane. This makes the sum reactions less favorable at higher temperatures. The driving force for the adduct formation and release of methane and ethane is the release reactions. The formation of adducts between $\mathrm{NH}_{3-\mathrm{x}}\left(\mathrm{CH}_{3}\right)_{\mathrm{x}}$ and $\mathrm{Al}\left(\mathrm{CH}_{3}\right)_{3}$ follows the same trends as the adduct formation with $\mathrm{Ga}\left(\mathrm{CH}_{3}\right)_{3}$; all details for adduct formation with $\mathrm{Al}\left(\mathrm{CH}_{3}\right)_{3}$ are given in the supporting information.

The alkane release mechanism from the adduct contains a migration of either a hydrogen (methane release) or a methyl (ethane release) from the nitrogen towards one of the methyl groups of $\mathrm{Ga}\left(\mathrm{CH}_{3}\right)_{3}$. Simultaneously the nitrogen and gallium atoms are pulled closer to form a covalent bond. The transition state barriers for the alkane release reactions are given in Table 4.5 and graphically in Figs. 4.10-4.11. The transition state enthalpy is almost constant for all reactions in the CVD temperature range and does also not depend on the number of methyl groups on the nitrogen. The activation enthalpy for the elimination of methane is lower than for the elimination of ethane. The activation free energy barrier of the eliminations has a higher dependence on temperature than the activation enthalpy has, except for reactions CM1 and CE3, and increases slightly with increasing temperatures. The activation free energy of methyl eliminations shows a larger 
dependence on the number of methyl ligands than the activation free energy of ethane eliminations does. As with the enthalpy barrier, the free energy barrier is higher for the elimination of ethane than for methane, demonstrating that the expected rate of ethane elimination is slower than the rate of methane elimination.

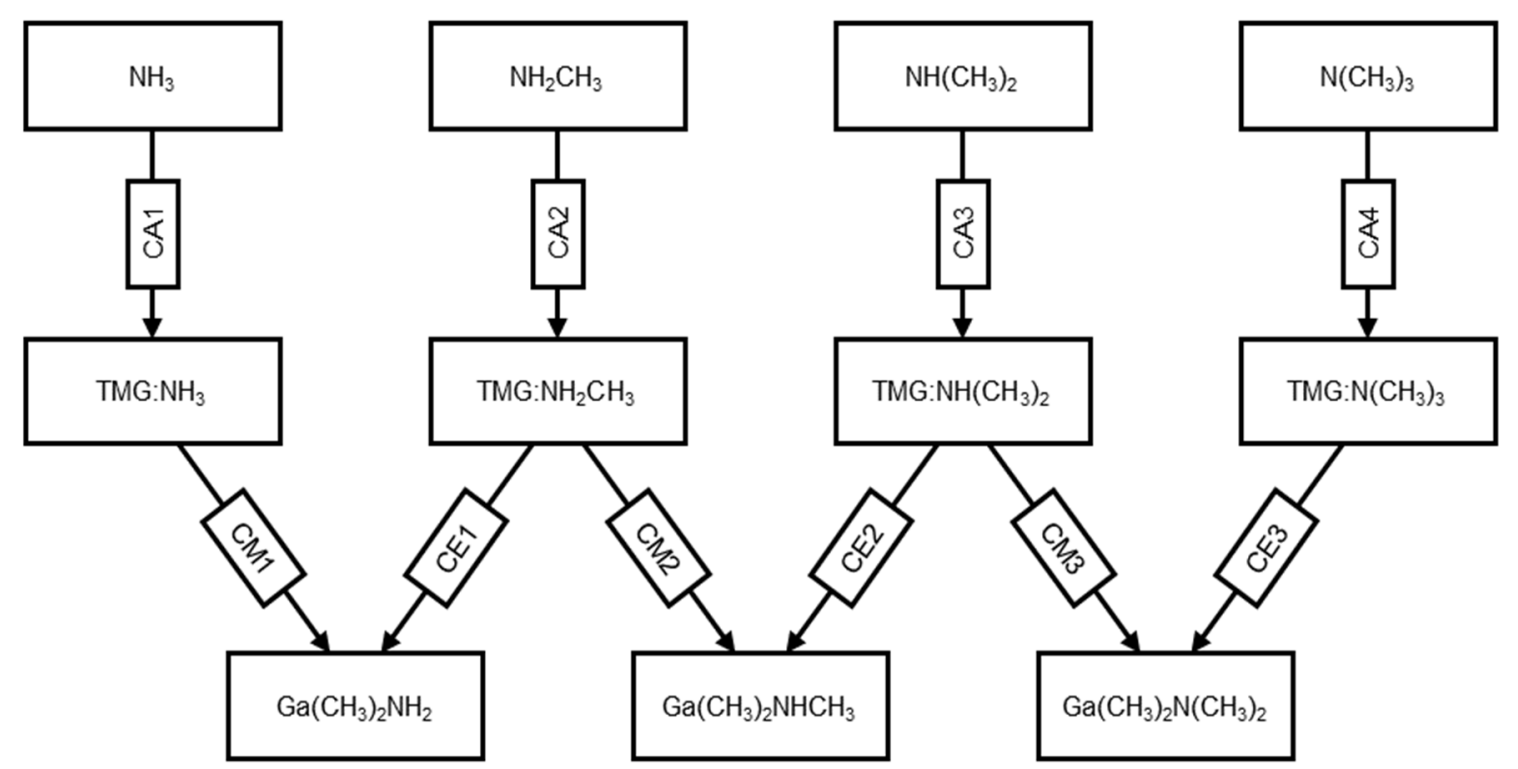

Scheme 4.2: Reaction pathway diagram of the adduct formation between $\mathrm{NH}_{3-}$ ${ }_{x}\left(\mathrm{CH}_{3}\right)_{x}$ and $\mathrm{Ga}\left(\mathrm{CH}_{3}\right)_{3}$ and subsequent alkane elimination reactions. Reactions $\mathrm{CAn}$ are the adduct formation, $\mathrm{CMn}$ are the release of methane, and CEn are the release of ethane. 
Table 4.4: Reaction enthalpies and free energies for adduct formation between $\mathrm{NH}_{3-}$ ${ }_{x}\left(\mathrm{CH}_{3}\right)_{\mathrm{x}}$ and $\mathrm{Ga}\left(\mathrm{CH}_{3}\right)_{3}$ and subsequent alkane elimination reactions at $\mathrm{CVD}$ conditions: $300{ }^{\circ} \mathrm{C}-1300{ }^{\circ} \mathrm{C}$ and 50 mbar. All energies are given in $\mathrm{kJ} / \mathrm{mol}$. Reaction indices refer to Scheme 4.2.

\begin{tabular}{|c|c|c|c|c|c|c|}
\hline \multirow{2}{*}{$\begin{array}{c}\text { Reaction } \\
\text { index }\end{array}$} & \multicolumn{6}{|c|}{$\Delta_{\mathrm{r}} \mathrm{H}^{\circ}$} \\
\hline & $300{ }^{\circ} \mathrm{C}$ & $500^{\circ} \mathrm{C}$ & $700{ }^{\circ} \mathrm{C}$ & $900{ }^{\circ} \mathrm{C}$ & $1100{ }^{\circ} \mathrm{C}$ & $1300{ }^{\circ} \mathrm{C}$ \\
\hline CA1 & -73.6 & -72.4 & -71.0 & -69.5 & -67.9 & -66.4 \\
\hline CA2 & -83.0 & -80.1 & -77.0 & -73.9 & -70.7 & -67.4 \\
\hline CA3 & -91.7 & -88.7 & -85.6 & -82.4 & -79.2 & -76.0 \\
\hline CA4 & -95.5 & -92.3 & -89.2 & -86.1 & -83.0 & -79.8 \\
\hline CM1 & 1.2 & -0.2 & -1.7 & -3.3 & -4.9 & -6.6 \\
\hline CM2 & 7.6 & 4.4 & 1.1 & -2.2 & -5.5 & -8.8 \\
\hline CM3 & 9.8 & 4.8 & -0.2 & -5.2 & -10.1 & -15.0 \\
\hline CE1 & -20.5 & -23.4 & -26.5 & -29.7 & -33.0 & -36.3 \\
\hline CE2 & 3.4 & 0.0 & -3.4 & -6.9 & -10.3 & -13.6 \\
\hline \multirow[t]{2}{*}{ CE3 } & 13.4 & 8.0 & 2.8 & -2.4 & -7.4 & -12.5 \\
\hline & \multicolumn{6}{|c|}{$\Delta_{\mathrm{r}} \mathrm{G}^{\circ}$} \\
\hline CA1 & 53.9 & 98.3 & 142.3 & 185.9 & 229.4 & 272.6 \\
\hline CA2 & 36.6 & 77.9 & 118.5 & 158.3 & 197.7 & 236.5 \\
\hline CA3 & 27.4 & 68.5 & 108.8 & 148.4 & 187.5 & 226.1 \\
\hline CA4 & 35.0 & 80.1 & 124.3 & 167.9 & 211.0 & 253.5 \\
\hline CM1 & -98.2 & -132.7 & -166.8 & -200.6 & -234.1 & -267.4 \\
\hline CM2 & -77.0 & -106.0 & -134.1 & -161.6 & -188.5 & -214.9 \\
\hline CM3 & -60.5 & -84.3 & -106.7 & -128.2 & -148.7 & -168.6 \\
\hline CE1 & -119.7 & -153.9 & -187.3 & -220.1 & -252.3 & -284.0 \\
\hline CE2 & -91.1 & -123.6 & -155.2 & -186.0 & -216.3 & -246.1 \\
\hline CE3 & -81.1 & -113.3 & -144.0 & -173.7 & -202.5 & -230.5 \\
\hline
\end{tabular}


Table 4.5: Activation enthalpies and free energies for alkane elimination from the adducts at CVD conditions: $300-1300^{\circ} \mathrm{C}$ and 50 mbar. All energies are given in $\mathrm{kJ} / \mathrm{mol}$. Reaction indices refer to Scheme 4.2.

\begin{tabular}{lcccccc}
\hline $\begin{array}{c}\text { Reaction } \\
\text { index }\end{array}$ & \multicolumn{5}{c}{$\Delta_{\mathrm{r}} \mathrm{H}^{\circ}$} & \\
\cline { 2 - 7 } & $300{ }^{\circ} \mathrm{C}$ & $500{ }^{\circ} \mathrm{C}$ & $700{ }^{\circ} \mathrm{C}$ & $900{ }^{\circ} \mathrm{C}$ & $1100{ }^{\circ} \mathrm{C}$ & $1300{ }^{\circ} \mathrm{C}$ \\
\hline CM1 & 144.8 & 145.8 & 146.8 & 147.8 & 148.7 & 149.4 \\
CM2 & 146.2 & 145.5 & 144.9 & 144.2 & 143.4 & 142.5 \\
CM3 & 148.0 & 147.3 & 146.6 & 145.9 & 145.1 & 144.2 \\
& & & & & & \\
CE1 & 386.6 & 387.1 & 386.8 & 386.1 & 385.0 & 383.8 \\
CE2 & 392.4 & 392.4 & 391.9 & 391.1 & 390.0 & 388.7 \\
CE3 & 388.2 & 388.0 & 387.3 & 386.4 & 385.3 & 384.0 \\
\cline { 2 - 6 } CM1 & 145.2 & 146.2 & 146.6 & 146.7 & 146.5 & 146.2 \\
CM2 & 151.4 & 155.5 & 158.8 & 162.4 & 166.0 & 169.8 \\
CM3 & 153.8 & 158.5 & 162.2 & 166.2 & 170.2 & 174.5 \\
& & & & & & \\
CE1 & 389.4 & 392.8 & 394.8 & 396.9 & 399.0 & 401.3 \\
CE2 & 393.7 & 395.5 & 396.5 & 397.6 & 398.9 & 400.3 \\
CE3 & 388.0 & 388.1 & 388.1 & 388.2 & 388.5 & 388.9 \\
\hline
\end{tabular}

\section{Surface interactions with GaN (0001)}

Adsorption reactions for both $\mathrm{NH}_{3-\mathrm{x}}\left(\mathrm{CH}_{3}\right)_{\mathrm{x}}$ and their decomposition products were modeled by allowing the adsorbate to displace a hydrogen at a GaN surface site, releasing a hydrogen radical.

$$
-\mathrm{H}+\mathrm{NH}_{\mathrm{x}-\mathrm{y}}\left(\mathrm{CH}_{3}\right)_{\mathrm{y}} \rightarrow \mathrm{HNH}_{\mathrm{x}-\mathrm{y}}\left(\mathrm{CH}_{3}\right)_{\mathrm{y}}+\mathrm{H} \cdot
$$


The reaction energies are presented in Table 4.6 and graphically in Figs. 4.10-4.11; Fig. 4.2 shows the geometries for the adsorption of the intact $\mathrm{NH}_{3-}$ ${ }_{\mathrm{x}}\left(\mathrm{CH}_{3}\right)_{\mathrm{x}}$ species on GaN (0001). In Table 4.6 the reactions are indexed as Dxy, where $\mathrm{x}$ is the total number of ligands attached to the nitrogen and $\mathrm{y}$ is the number of methyl ligands. Reaction DRE is the adsorption of the rearranged methanimine. While the adsorptions of $\mathrm{NH}_{3}$ and $\mathrm{NH}_{2} \mathrm{CH}_{3}$ are exothermic at the CVD temperatures, undecomposed $\mathrm{NH}\left(\mathrm{CH}_{3}\right)_{2}$ and $\mathrm{N}\left(\mathrm{CH}_{3}\right)_{3}$ have a positive reaction enthalpy. The adsorption of all undecomposed species has a positive free energy of adsorption at all CVD temperatures and thus these are not likely to adsorb significantly. The reaction free energy also increases with the temperature for adsorption of all undecomposed species. The computed N - Ga distances and geometries indicate that the adsorption of $\mathrm{N}\left(\mathrm{CH}_{3}\right)_{3}$ is hindered by steric repulsion due to the methyl groups.

For the first decomposition products, the adsorptions at CVD conditions are exothermic and exergonic, except for $\mathrm{N}\left(\mathrm{CH}_{3}\right)_{2}$ which is endergonic at high CVD temperature. A lower number of methyl groups on the nitrogen gives a lower adsorption free energy. At higher temperatures, the adsorptions have higher reaction free energies and the effect of temperature is larger as the number of methyl groups is increased. The overall lowest reaction free energy is for the

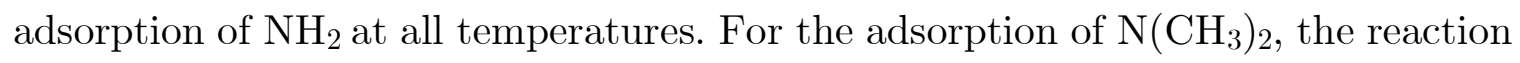


changes from exergonic at lower temperatures to endergonic at temperatures between $900-1100{ }^{\circ} \mathrm{C}$. All other reaction free energies are negative indicating favorable reactions.

As with the first decomposition products, all desorptions of second decomposition products are exothermic. The adsorption of $\mathrm{NH}$ and $\mathrm{NCH}_{3}$ are exergonic at lower CVD temperatures and $\mathrm{NCH}_{3}$ becomes endergonic above 900$1100{ }^{\circ} \mathrm{C}$. The reaction free energy is higher for the adsorption of $\mathrm{NCH}_{3}$ than for $\mathrm{NH}$ at all CVD temperatures. The adsorption of the nitrogen radical is both exothermic and exergonic and is less favorable than the adsorption of $\mathrm{NH}$ but more favorable than the adsorption of $\mathrm{N}\left(\mathrm{CH}_{3}\right)$, though the free energy approaches that of $\mathrm{NH}$ as temperature is increased. The adsorption of $\mathrm{NHCH}_{2}$ is has an adsorption enthalpy close to zero at all CVD conditions and the high adsorption free energy makes adsorption unfavorable.

A radical precursor species is necessary to allow exchange at the hydrogenated GaN surface. The reaction is more thermodynamically likely with a lower number of methyls (i.e., higher number of hydrogen bonded to the precursor nitrogen). In the CVD temperature range, the increase in $\Delta_{\mathrm{r}} \mathrm{G}$ is more significant for species with a greater number of methyl groups than for ammonia-derived gaseous reactants. 

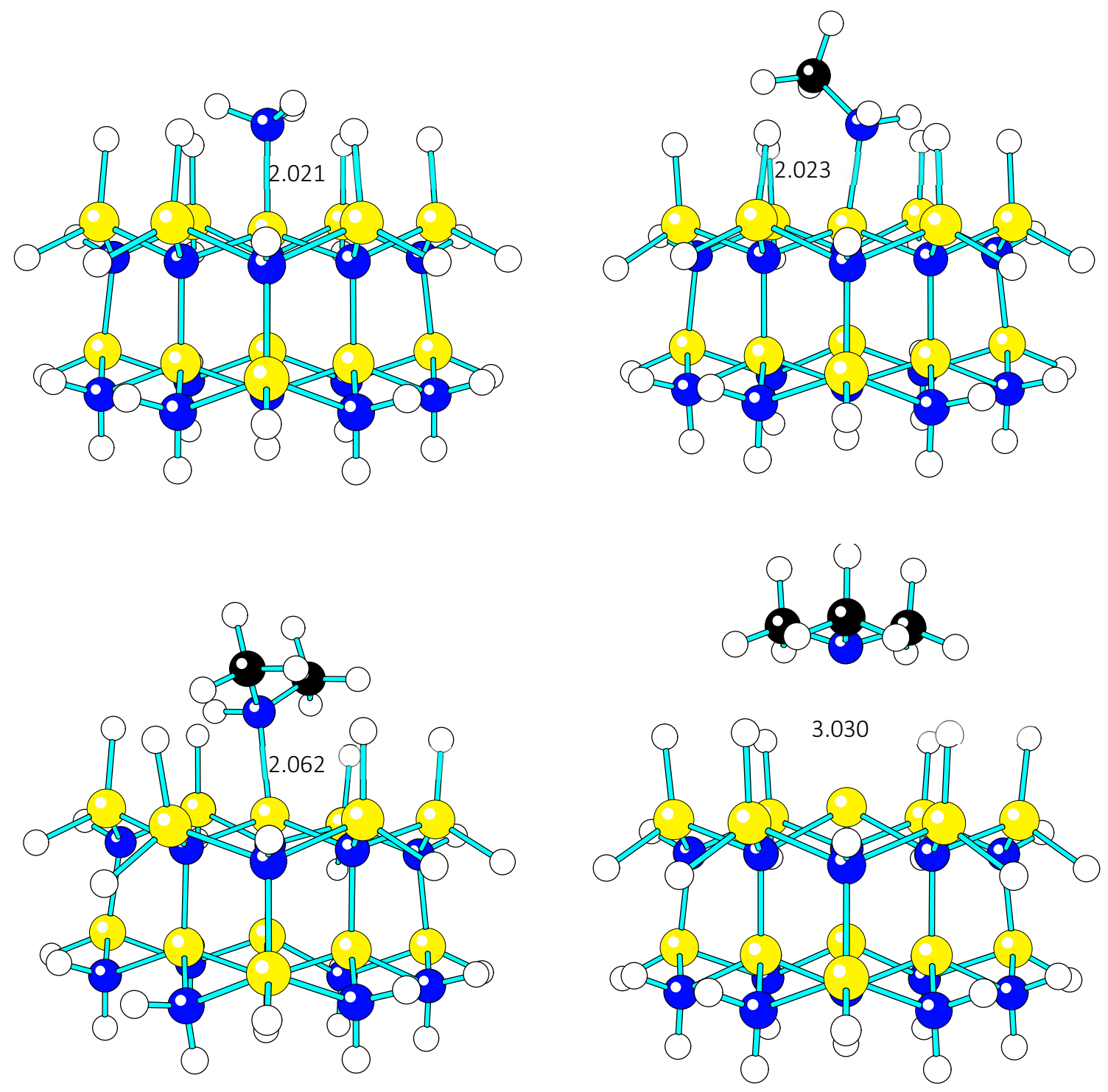

Figure 4.2: Optimized geometries for the D30 - D33 adsorptions of undecomposed precursors at the GaN (0001) surface. The distance between the Ga at the adsorption site and the $\mathrm{N}$ in the precursor is given in Angströms. Yellow atoms are gallium, blue nitrogen, black carbon and white hydrogen. 
Table 4.6: Reaction enthalpies and free energies for the reaction of ammonia and methylamines with the H-terminated GaN (0001) surface at CVD conditions $\left(300^{\circ} \mathrm{C}-1300^{\circ} \mathrm{C}\right.$ at $\left.50 \mathrm{mbar}\right)$. The reactions are indexed as Dxy, where $\mathrm{x}$ is the total number of ligands attached to the nitrogen and $\mathrm{y}$ is the number of methyl ligands. Reaction DRE is the adsorption of the rearranged methanimine. All values are given in $\mathrm{kJ} / \mathrm{mol}$.

\begin{tabular}{|c|c|c|c|c|c|c|}
\hline \multirow{2}{*}{$\begin{array}{l}\text { Reaction } \\
\text { index }\end{array}$} & \multicolumn{6}{|c|}{$\Delta_{\mathrm{r}} \mathrm{H}^{\circ}$} \\
\hline & $300{ }^{\circ} \mathrm{C}$ & $500{ }^{\circ} \mathrm{C}$ & $700{ }^{\circ} \mathrm{C}$ & $900{ }^{\circ} \mathrm{C}$ & $1100{ }^{\circ} \mathrm{C}$ & $1300{ }^{\circ} \mathrm{C}$ \\
\hline D30 & -11.6 & -9.5 & -7.3 & -5.2 & -3.0 & -0.9 \\
\hline D31 & -24.0 & -23.3 & -22.7 & -22.2 & -21.8 & -21.3 \\
\hline D32 & 4.4 & 5.1 & 5.6 & 6.0 & 6.3 & 6.6 \\
\hline D33 & 79.8 & 79.6 & 78.9 & 77.8 & 76.6 & 75.3 \\
\hline D20 & -256.8 & -254.7 & -252.8 & -251.0 & -249.3 & -247.6 \\
\hline D21 & -228.6 & -226.6 & -224.8 & -223.1 & -221.4 & -219.7 \\
\hline D22 & -196.5 & -196.2 & -196.1 & -196.0 & -196.0 & -196.0 \\
\hline D10 & -201.4 & -201.7 & -202.2 & -202.8 & -203.5 & -204.2 \\
\hline D11 & -171.2 & -172.1 & -173.3 & -174.6 & -176.1 & -177.5 \\
\hline DRE & -3.7 & 0.0 & 3.1 & 5.7 & 8.1 & 10.3 \\
\hline \multirow[t]{2}{*}{ Doo } & -145.0 & -144.8 & -145.0 & -145.4 & -145.9 & -146.4 \\
\hline & \multicolumn{6}{|c|}{$\Delta_{\mathrm{r}} \mathrm{G}^{\circ}$} \\
\hline D30 & 38.3 & 55.3 & 71.8 & 87.9 & 103.6 & 119.0 \\
\hline D31 & 49.6 & 75.2 & 100.6 & 125.8 & 151.0 & 176.2 \\
\hline D32 & 87.9 & 117.0 & 145.9 & 174.6 & 203.4 & 232.1 \\
\hline D33 & 144.1 & 166.6 & 189.1 & 211.9 & 234.8 & 258.0 \\
\hline D20 & -205.6 & -188.0 & -171.0 & -154.3 & -138.0 & -121.9 \\
\hline D21 & -163.2 & -140.7 & -118.7 & -97.1 & -75.7 & -54.6 \\
\hline D22 & -107.3 & -76.3 & -45.3 & -14.3 & 16.7 & 47.7 \\
\hline D10 & -148.6 & -130.2 & -111.6 & -93.0 & -74.2 & -55.3 \\
\hline D11 & -96.9 & -70.9 & -44.5 & -17.9 & 8.9 & 35.9 \\
\hline DRE & 38.3 & 52.3 & 65.4 & 78.0 & 90.1 & 101.9 \\
\hline D00 & -111.7 & -100.1 & -88.5 & -76.9 & -65.2 & -53.4 \\
\hline
\end{tabular}


In summary, the computational study shows that the gas phase chemistry for decomposition of $\mathrm{NH}_{3-\mathrm{x}}\left(\mathrm{CH}_{3}\right)_{\mathrm{x}}$ becomes more thermodynamically favorable with more methyl groups in the amine precursor. Adduct formation is more favorable with fewer methyl groups in the amine precursor, and the subsequent release of methane or ethane is less favorable as the number of methyl groups is increased. Overall, the gas phase chemistry becomes more favored with higher temperatures. The surface chemistry for adsorption of the $\mathrm{NH}_{3-\mathrm{x}}\left(\mathrm{CH}_{3}\right)_{\mathrm{x}}$ becomes more favorable for decomposition products with fewer methyl groups and with lower temperatures. This suggests that $\mathrm{N}\left(\mathrm{CH}_{3}\right)_{3}$ should be the best nitrogen precursor for gas phase chemistry rendering a higher concentration of more reactive species with less nitrogen species in adducts with gallium species. However, $\mathrm{NH}_{3}$ is the most favored nitrogen precursor for surface chemistry as the more methyl groups on the nitrogen atoms, the higher the steric hindrance for chemisorption seems to be.

\section{CVD experiments of GaN using $\mathrm{NH}_{3-\mathrm{x}}\left(\mathrm{CH}_{3}\right)_{\mathrm{x}}$ and $\mathrm{Ga}\left(\mathrm{CH}_{3}\right)_{3}$}

CVD of of GaN was first undertaken with $\mathrm{NH}_{3}$ and $\mathrm{Ga}\left(\mathrm{CH}_{3}\right)_{3}$ to use as a reference. With a $\mathrm{N} / \mathrm{Ga}$ ratio of 570 and reactor temperatures of $850{ }^{\circ} \mathrm{C}$ and $1000{ }^{\circ} \mathrm{C}$, this process resulted in crystalline GaN films with somewhat rough surfaces (Fig. 4.3). The deposition rate was found to be $44 \mathrm{~nm} / \mathrm{min}$ at $1000{ }^{\circ} \mathrm{C}$ and $37 \mathrm{~nm} / \mathrm{min}$ at 850 ${ }^{\circ} \mathrm{C}$. 

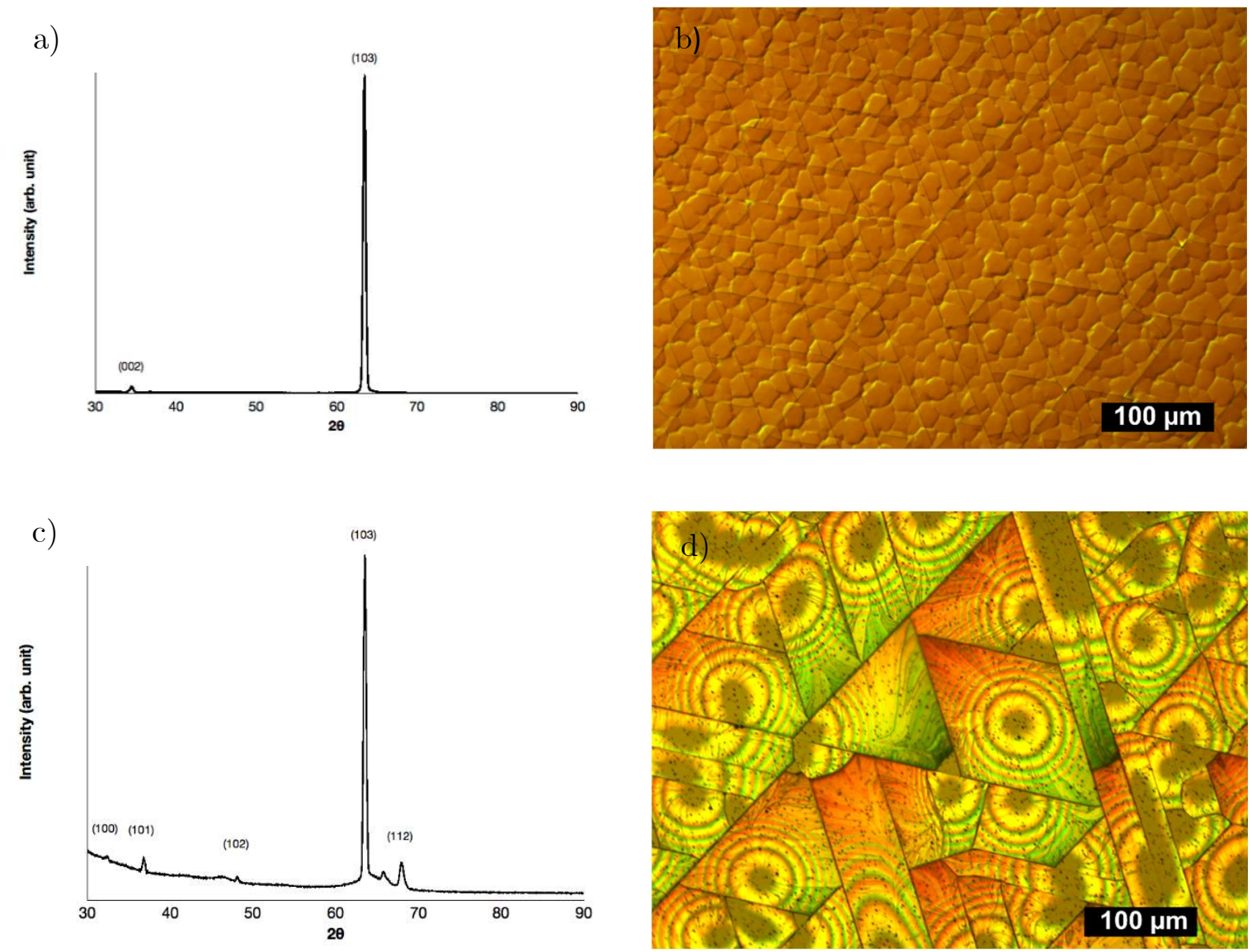

Figure 4.3: GaN films grown by CVD using $\mathrm{Ga}\left(\mathrm{CH}_{3}\right)_{3}$ and $\mathrm{NH}_{3}$ with $\mathrm{N} / \mathrm{Ga}=570$. Deposition at $1000{ }^{\circ} \mathrm{C}$ in panels a) and b) and at $850{ }^{\circ} \mathrm{C}$ in panels c) and d) afforded crystalline GaN films, as seen from GIXRD with a rough surface, imaged by optical microscopy.

The same experimental conditions with a reactor temperature of $1000{ }^{\circ} \mathrm{C}$ and $\mathrm{N} / \mathrm{Ga}=570$ were then used for CVD experiments with $\mathrm{NH}_{2} \mathrm{CH}_{3}, \mathrm{NH}\left(\mathrm{CH}_{3}\right)_{2}$ and $\mathrm{N}\left(\mathrm{CH}_{3}\right)_{3}$. None of these amines yielded any film by SEM or XRD under these conditions. CVD experiments at $850{ }^{\circ} \mathrm{C}$ using $\mathrm{NH}_{2} \mathrm{CH}_{3}$ and $\mathrm{N}\left(\mathrm{CH}_{3}\right)_{3}$ with $\mathrm{N} / \mathrm{Ga}=$ 570 were performed to probe the possibility of lower CVD temperatures for GaN by $\mathrm{NH}_{3-\mathrm{x}}\left(\mathrm{CH}_{3}\right)_{\mathrm{x}}$. These experiments yielded Ga metal droplets (Figure 4.4). 

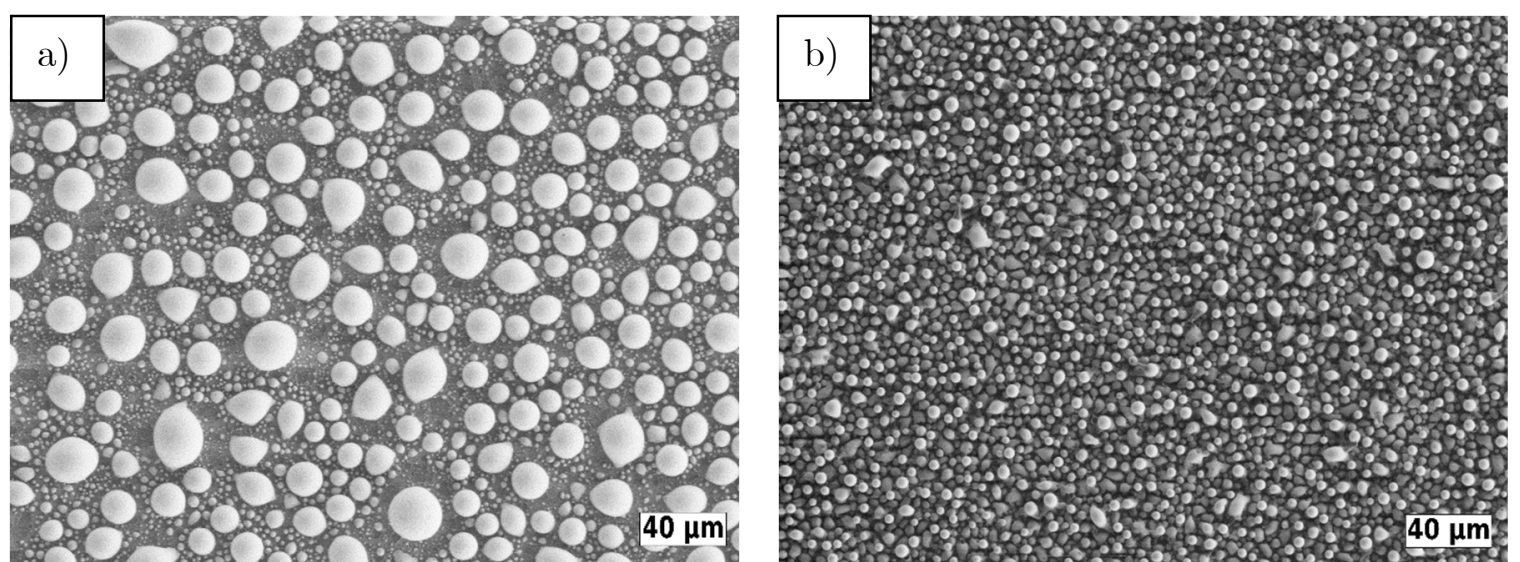

Figure 4.4: SEM surface micrographs from CVD samples grown at $850{ }^{\circ} \mathrm{C}$ using $\mathrm{Ga}\left(\mathrm{CH}_{3}\right)_{3}$ and a) $\mathrm{NH}_{2} \mathrm{CH}_{3}$ and b) $\mathrm{N}\left(\mathrm{CH}_{3}\right)_{3}$ using $\mathrm{N} / \mathrm{Ga}=570$.

Further attempts to form GaN were undertaken using $\mathrm{N}\left(\mathrm{CH}_{3}\right)_{3}$ and $\mathrm{Ga}\left(\mathrm{CH}_{3}\right)_{3}$ The $\mathrm{N} / \mathrm{Ga}$ ratio was tuned by varying the flow of $\mathrm{N}\left(\mathrm{CH}_{3}\right)_{3}$ while keeping the flow of $\mathrm{Ga}\left(\mathrm{CH}_{3}\right)_{3}$ constant at an intermediate deposition temperature of 925 ${ }^{\circ} \mathrm{C}$. These experiments also resulted in the formation of droplets with the additional growth of cones (Fig. 4.5). The number of the droplets and cones varied with the $\mathrm{N} / \mathrm{Ga}$ ratio; a high $\mathrm{N} / \mathrm{Ga}$ ratio (i.e., higher flow of $\mathrm{N}\left(\mathrm{CH}_{3}\right)_{3}$ ) resulted in a lower density of droplets and cones as seen by SEM (Fig. 4.5). This was also seen by the naked eye as the samples from CVD with a lower N/Ga appeared greyer and more metallic than the samples from CVD with higher $\mathrm{N} / \mathrm{Ga}$, which had a shiny appearance on the transparent greenish $\mathrm{SiC}$ substrate. The grey coating on the samples could easily be scratched. From EDS (Fig. 4.15), the droplets were pure gallium, while the cones consisted of gallium with obvious amounts of nitrogen and carbon. GIXRD of the sample (Fig. 4.16) show an indication of crystalline gallium in its orthorhombic structure and possible weak XRD peaks from crystalline GaN. 


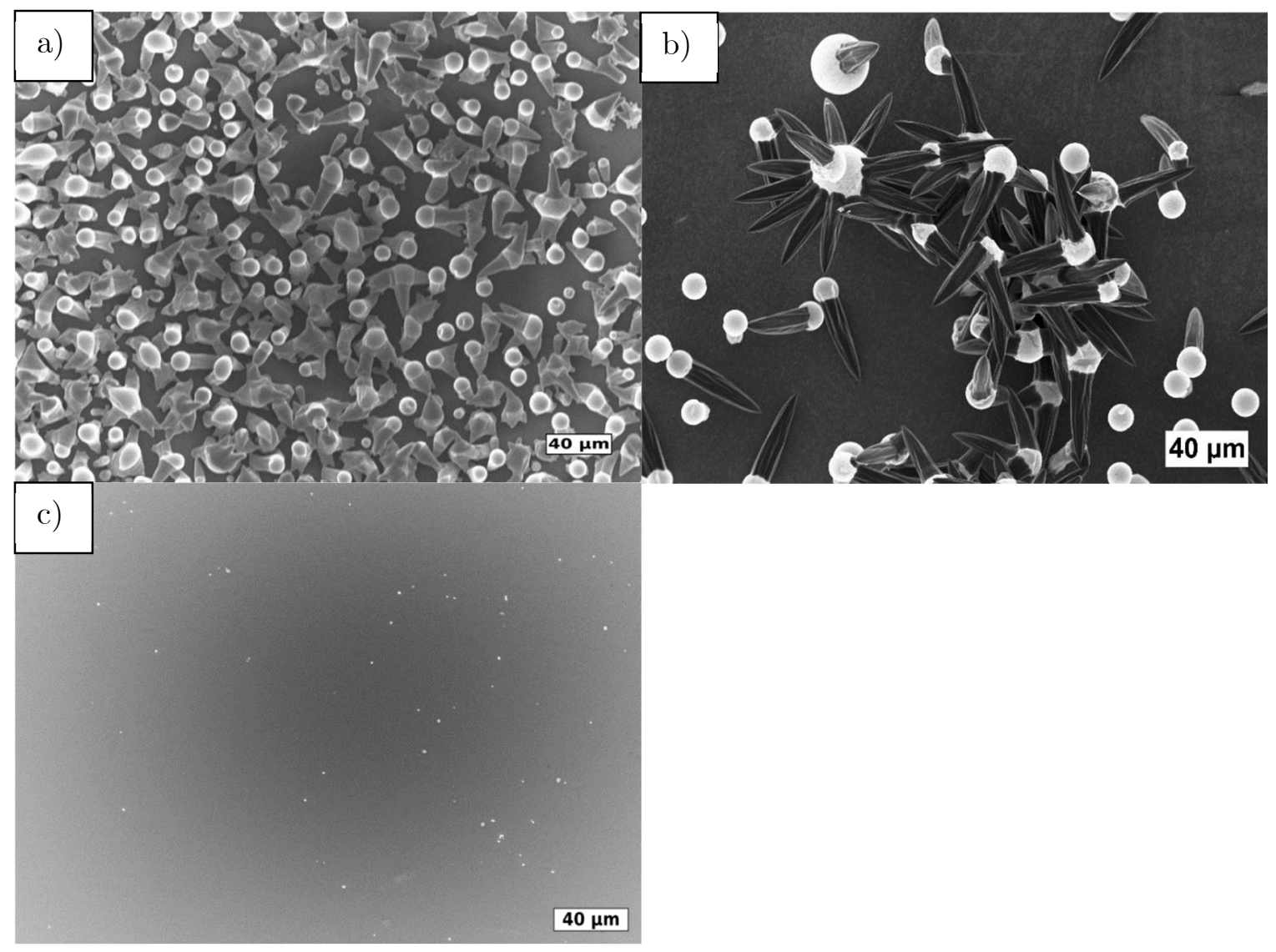

Figure 4.5: SEM surface micrographs from CVD samples grown at $925{ }^{\circ} \mathrm{C}$ from $\mathrm{Ga}\left(\mathrm{CH}_{3}\right)_{3}$ and $\mathrm{N}\left(\mathrm{CH}_{3}\right)_{3}$ using a) $\mathrm{N} / \mathrm{Ga}=275$ and b) $\mathrm{N} / \mathrm{Ga}=570$ and c) $\mathrm{N} / \mathrm{Ga}=$ 1100 .

To further study how the concentration of amine affected the deposition, experiments with a N/Ga fixed at 570 and changing the flows, and thereby the partial pressures, of both $\mathrm{N}\left(\mathrm{CH}_{3}\right)_{3}$ and $\mathrm{Ga}\left(\mathrm{CH}_{3}\right)_{3}$ were performed (Fig. 4.6). From these experiments a higher partial pressure of amine, coupled with a higher partial pressure of $\mathrm{Ga}\left(\mathrm{CH}_{3}\right)_{3}$, rendered larger droplets and fewer cones. 


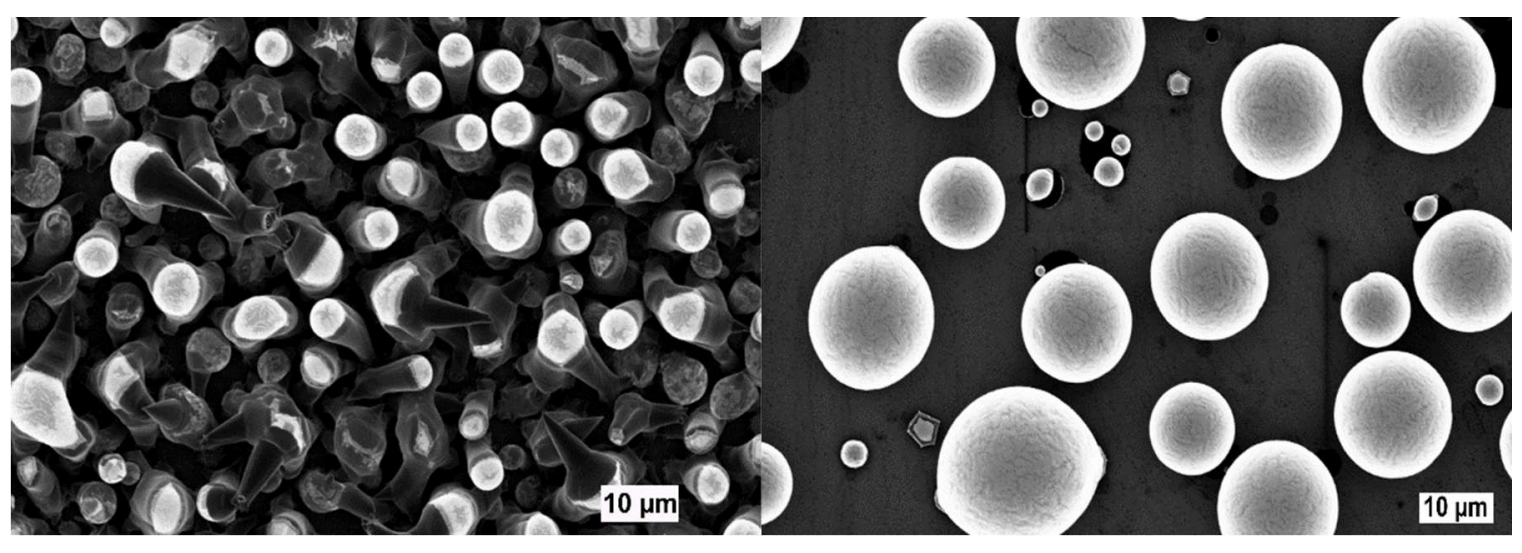

Figure 4.6: SEM surface micrographs from CVD samples grown at $925{ }^{\circ} \mathrm{C}$ using $\mathrm{Ga}\left(\mathrm{CH}_{3}\right)_{3}$ and $\mathrm{N}\left(\mathrm{CH}_{3}\right)_{3}$ with $\mathrm{N} / \mathrm{Ga}=570$ and a) low precursor flow $\left(\mathrm{N}\left(\mathrm{CH}_{3}\right)_{3}\right.$ at a partial pressure of 2 mbar) and b) high precursor flow $\left(\mathrm{N}\left(\mathrm{CH}_{3}\right)_{3}\right.$ at a partial pressure of $8 \mathrm{mbar})$.

\section{Discussion}

While the computational study shows that the amines will decompose more easily than ammonia and thereby be more abundant nitrogen precursors in the gas phase at thermodynamic equilibrium for CVD, the experimental study demonstrated that methylamines cannot replace ammonia in CVD of GaN. Rather, methylamines seem to be poor nitrogen precursors as no contiguous GaN films could be grown using them in this study. This is in line with the computational results that showed that methylamines interacted less favorably than amines with the GaN surface (i.e. higher $\Delta_{\mathrm{r}} \mathrm{G}$ in Table 4.6), suggesting that surface chemistry rather than gas phase chemistry is the limiting factor to growing high quality GaN films. Specifically, the exchange of an activated gas phase radical for a surface hydrogen shows less of a thermodynamic driving force as the number of methyl groups increase. This is not 
due to a difference in the $\mathrm{N}-\mathrm{H}$ vs. N-Me bond strength (as was originally supposed), but rather due to a difference in radical chemisorption at a surface gallium site. Another factor to consider is that the bimolecular decomposition rates are lower for methylamines than for amines due to higher activation energies (Table 4.3), which make the methylamine precursor concentrations lower than expected if equilibrium has not been reached in the CVD process. It can also be noted that potentially detrimental adduct derivatives can be formed after both methane and ethane elimination (Scheme 2) for most of the $\mathrm{Ga}\left(\mathrm{CH}_{3}\right)_{3}$-methylamine adducts, but only by methane elimination for the $\mathrm{Ga}\left(\mathrm{CH}_{3}\right)_{3}$-ammonia adducts. This could indicate that carbon released as more reactive ethane, rather than less reactive methane, is detrimental to the CVD process of GaN. As our attempts to achieve a continuous GaN film, both by varying $\mathrm{N} / \mathrm{Ga}$ (through changing the partial pressure of the trimethylamine) and by varying the partial pressures of both trimethylamine and trimethylgallium while keeping a fixed $\mathrm{N} / \mathrm{Ga}$, all resulted in formation of droplets and cones. And, a higher partial pressure of the amine, which should be beneficial for adduct formation, always resulted in less growth of cones and formation of larger droplets. Furthermore, EDX mapping of the cones (Fig. 4.15) shows a significant carbon signal, likely originating from carbon adsorbed on the surface, as the solubility of carbon is very low in both Ga ${ }^{173}$ and GaN. ${ }^{174}$ This can be interpreted as the trimethylamine or its gas phase reaction products, such as ethane, hindering the growth of GaN, leaving droplets of metallic gallium on the 
surface. This reasoning can be extended to explain why no GaN films were grown using any of the methylamines: the amines or their reaction products could be passivating GaN surfaces, forcing the enhanced formation of metallic gallium droplets.

The presence of metallic droplets appears to favor a VLS-type growth mechanism of cones rather than films. The morphology from SEM, Figs. 4.5b and EDS mapping Fig. 4.15, suggests that the metallic gallium droplet is growing while also seeding growth of the cone, causing the growing cone to continually increase in diameter while it grows in length (Fig. 4.7) This shows similar behavior to the growth of $\mathrm{Ga}_{2} \mathrm{O}_{3}$ cones using an amidinate precursor and water. ${ }^{60}$ The material comprising the cone is not fully understood; as no peaks related to gallium oxide could be seen in the GIXRD pattern (Fig. 4.16) and considering the very weak tendency for gallium to form carbides $^{175}$ and that nitrogen was detected in the cones by EDX (Fig. 4.15), a probable material in the cones is an X-ray amorphous gallium nitride. The high amount of carbon detected on the cones in EDX (Fig. 4.15) could disturb the nitride forming process, leading to an X-ray amorphous gallium nitride structure. 


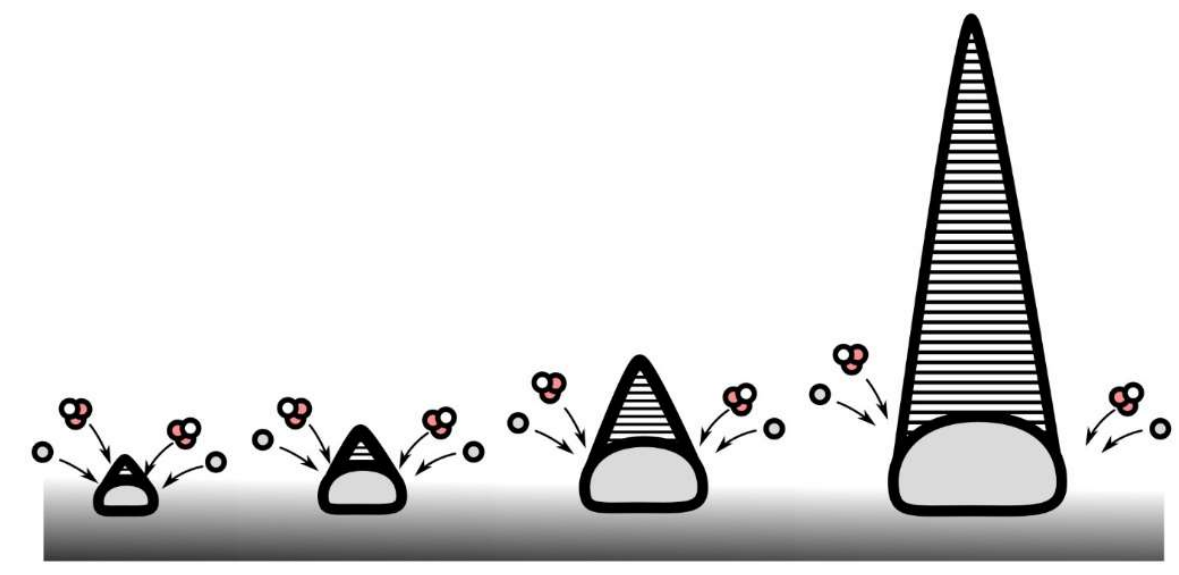

Figure 4.7: Schematics of a possible growth mechanism for the observed cones.

Although it was observed that low amine flow led to the formation of droplets and cones, and high amine flow led to no deposited species at all, it is unclear what the cause of this is. The formation of gallium metal droplets at the surface occurs more readily at low amine/Ga precursor ratio, N/Ga $=275$. Perhaps a gallium species, most likely $\mathrm{GaCH}_{3}$ with minor contribution from atomic Ga, Error! Bookmark not defined. reaches the surface and deposits gallium metal by reductive elimination of ethane:

$$
2 \mathrm{GaCH}_{3} \rightarrow 2 \mathrm{Ga}^{0}+\mathrm{C}_{2} \mathrm{H}_{6}
$$

This would be an irreversible process as there are no oxidizing agents present in the CVD gas mixture. Under low and intermediate amine flow, GaN could then nucleate on the growing droplet surface. Under high amine flow, where there was no apparent GaN formation, the gallium metal could be etched by the overpressure of amine by a subsequent oxidative addition: 
$2 \mathrm{n} \mathrm{Ga}^{0}+3 \mathrm{n} \mathrm{N}\left(\mathrm{CH}_{3}\right)_{3} \rightarrow$ n-x $\left(\mathrm{CH}_{3}\right)_{2} \mathrm{Ga}^{\mathrm{IIIN}}\left(\mathrm{CH}_{3}\right)_{2}+\mathrm{x} \mathrm{CH} \mathrm{CHa}^{\mathrm{III}}\left(\mathrm{N}\left(\mathrm{CH}_{3}\right)_{2}\right)_{2}$

Another explanation for the enhanced formation of $\mathrm{Ga}$ droplets is a reversible blocking of surface sites by the methylamines reaction products, partially preventing growth of $\mathrm{GaN}$, forcing incoming gallium species to adsorb in a such a way that reductive elimination of alkanes is favored.

\subsection{Summary and conclusions}

From quantum chemical computations we find that methylamines show a higher reactivity in the gas phase chemistry of a CVD process for group 13 nitrides than (the currently used precursor) ammonia, while ammonia and its decomposition products show a higher reactivity towards a GaN surface than methylamines and their decomposition products. CVD experiments where ammonia was replaced by $\mathrm{NH}_{3-\mathrm{x}}\left(\mathrm{CH}_{3}\right)_{\mathrm{x}}$ with $\mathrm{x}=1,2$ or 3 , did not deposit continuous films, and instead the experiments rendered micrometer sized gallium droplets. At some experimental conditions, cone shaped outgrowths from the droplets, probably consisting of $\mathrm{X}$ ray amorphous GaN, were observed. This study shows that in the search to replace ammonia with a more efficient nitrogen precursor in CVD of the group 13 nitrides, allowing for a N/13 ratio closer to unity and lower CVD temperatures, the surface chemistry of the reaction fragments is likely more important than a more reactive 
gas phase chemistry. The results also suggest that care should be taken to avoid formation of too-reactive hydrocarbon fragments of any new nitrogen precursor.

\subsection{Acknowledgements}

This project was founded by the Swedish foundation for Strategic Research through the project "Time-resolved low temperature CVD for III-nitrides" (SSF-RMA 150018). STB and SCB acknowledge the Vinnova VINNMER Marie Curie incoming mobility program for funding for a sabbatical and research visits to Linköping University (Vinnova grant 2015-03714). HP and STB are very grateful for the networking support provided from the COST Action MP1402 'Hooking together European research in atomic layer deposition (HERALD) supported by COST (European Cooperation in Science and Technology). LO acknowledges financial support from the Swedish Government Strategic Research Area in Materials Science on Functional Materials at Linköping University (Faculty Grant SFO Mat LiU No. 2009 00971). Supercomputing resources were provided by the Swedish National Infrastructure for Computing (SNIC) and the Swedish National Supercomputer Centre (NSC). Urban Forsberg and Chih-Wei Hsu are gratefully acknowledged for technical assistance with the CVD reactor. 


\subsection{Supporting information}
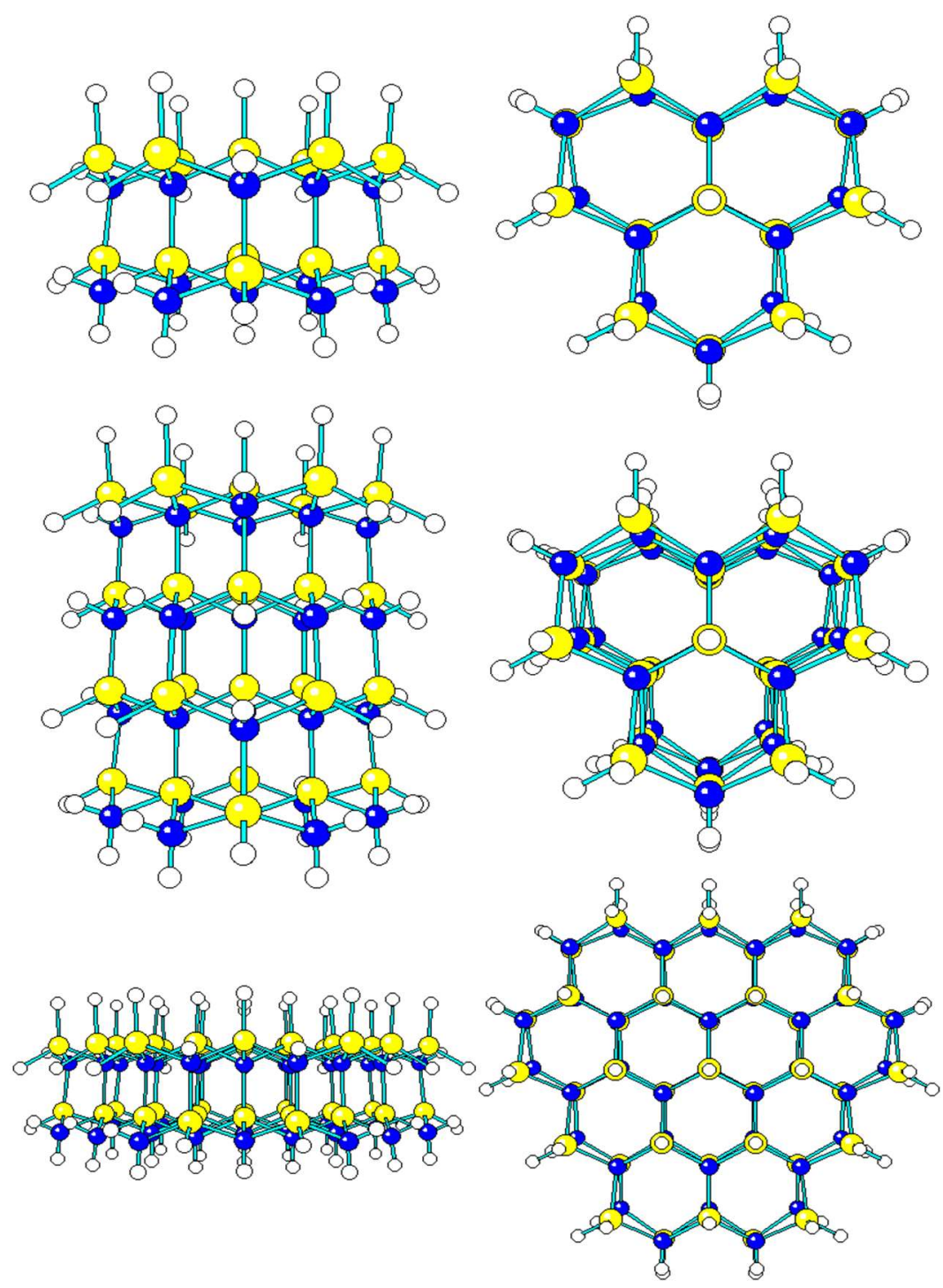

Figure 4.8: The three hydrogen terminated gallium nitride cluster used to evaluate the cluster size; top the smaller $(\mathrm{GaN}){ }_{13} \mathrm{H}_{32}$, middle the two layer $(\mathrm{GaN})_{26} \mathrm{H}_{50}$ and bottom the larger $(\mathrm{GaN})_{37} \mathrm{H}_{68}$. Viewed along the symmetry axis on the left and from the side at the right. Yellow atoms are gallium, blue are nitrogen and white are hydrogen. 


\section{Evaluation of the G4 method}

The bond energy for the gallium-carbon bond was calculated at both G4 and $\operatorname{CCSD}(\mathrm{T}) /$ aug-cc-pVTZ level of theory as an evaluation of the G4 method. The bond energy was estimated by the electronic energy of the reaction $\mathrm{Ga}\left(\mathrm{CH}_{3}\right)_{3} \rightarrow$ $\mathrm{Ga}\left(\mathrm{CH}_{3}\right)_{2}+\mathrm{CH}_{3}$. Geometries of the reactant and products were optimized at the B3LYP/6-31G(2df,p) level of theory. The reaction energy calculated with G4 was $317 \mathrm{~kJ} / \mathrm{mol}$ while the energy from $\operatorname{CCSD}(\mathrm{T})$ was $328 \mathrm{~kJ} / \mathrm{mol}$.

\section{Size of surface model cluster}

The size of the cluster was investigated by computation of the reaction energy of the adsorption of $\mathrm{NH}_{3}$ onto a hydrogen terminated cluster, releasing a hydrogen molecule. The reaction energy was computed at B3LPY/6-31G level of theory. Three different clusters were investigated; a smaller $(\mathrm{GaN}){ }_{13} \mathrm{H}_{32}$, a two-layer $(\mathrm{GaN})_{26} \mathrm{H}_{50}$ and a larger $(\mathrm{GaN}){ }_{37} \mathrm{H}_{68}$. The energy for adsorption was calculated to $-54.6 \mathrm{~kJ} / \mathrm{mol}$ for the smaller cluster, $-52.9 \mathrm{~kJ} / \mathrm{mol}$ for the two-layer cluster and $54.8 \mathrm{~kJ} / \mathrm{mol}$ for the larger cluster. Since the difference in energy (less than 2 $\mathrm{kJ} / \mathrm{mol}$ ) was very small between the different clusters and that the smaller cluster allowed us to use a larger basis set we opted to use the smaller cluster in the computational study. 


\section{Handling of internal rotations}

The assumption of treating internal rotations as vibration modes where investigated for reaction $\mathrm{BC} 2\left(\mathrm{NH}\left(\mathrm{CH}_{3}\right)_{2}+\mathrm{H} \rightarrow \mathrm{NH}\left(\mathrm{CH}_{3}\right)_{2} \mathrm{H}^{\ddagger} \rightarrow \mathrm{NHCH}_{3}+\mathrm{CH}_{4}\right)$.

Frequency calculations where performed at the B3LYP/6-31G(2df,p) level of theory where the frequencies were scaled by a factor 0.9854 in the same manner as in the G4-method. The enthalpy and free energy of the reaction and transition state when the internal rotations were treated as vibrations were compared to energies obtained by correcting for the rotations. As seen in Fig. 4.9 the largest difference was for the free energy at higher temperatures, but the difference in both enthalpy and free energy was less than $3.5 \mathrm{~kJ} / \mathrm{mol}$ which is in the chemical accuracy.
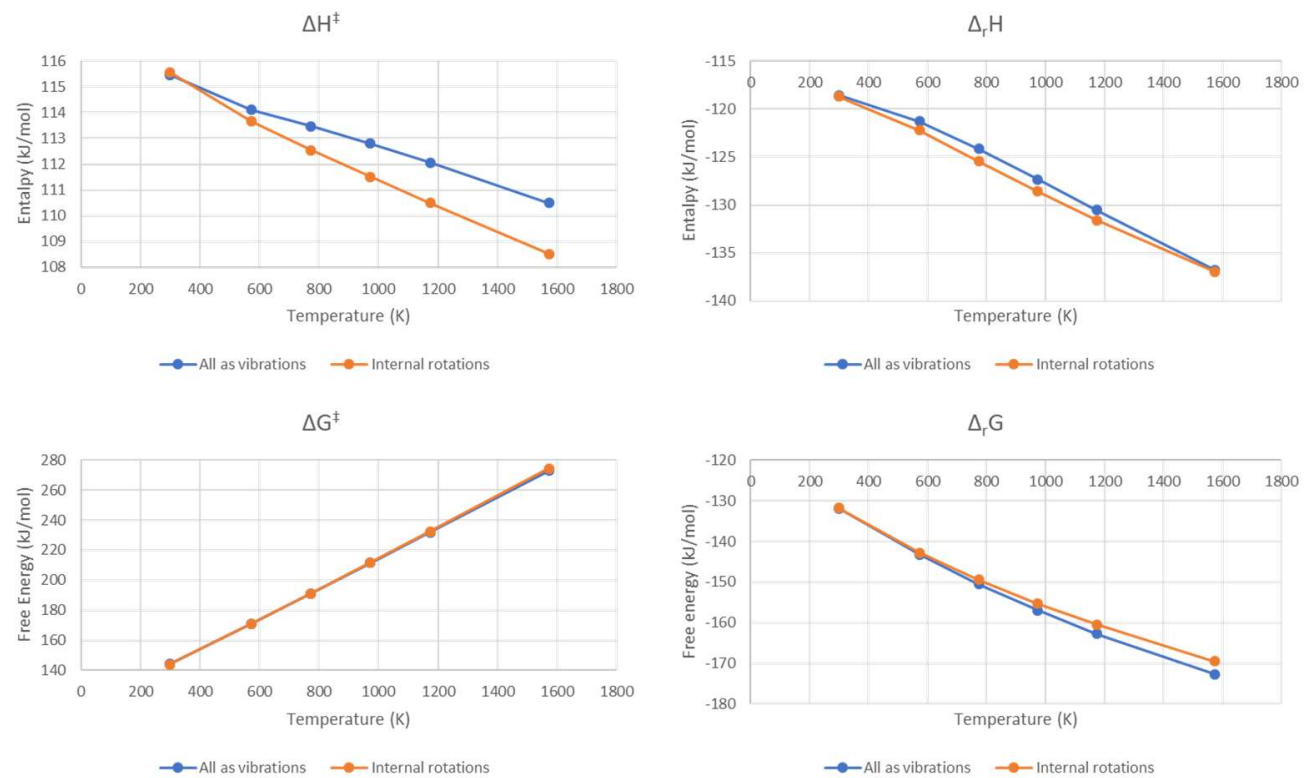

Figure 4.9: Enthalpy and free energy of transition state and reaction for BC2. Blue without correction for internal rotations and orange with corrections. 

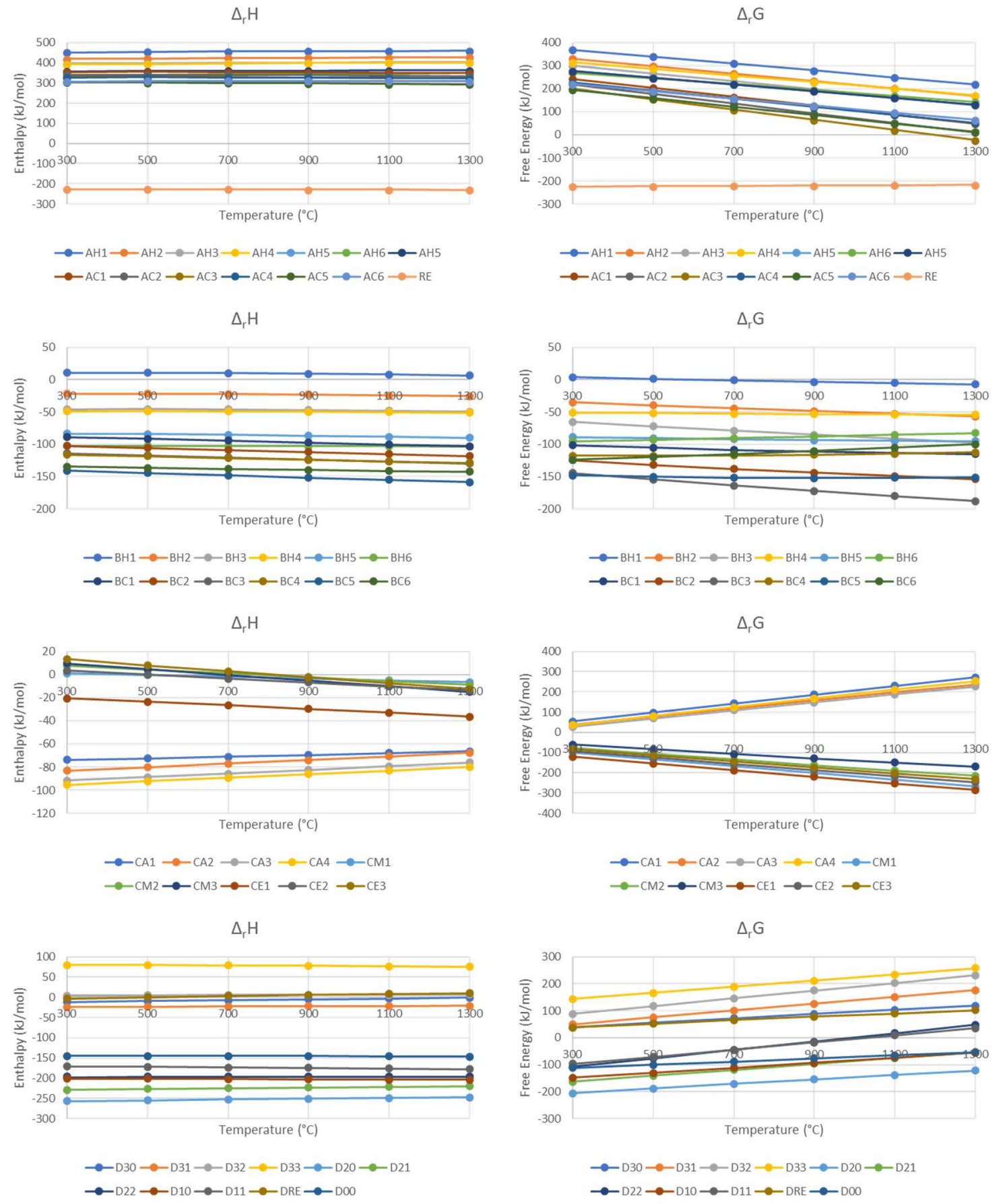

Figure 4.10: Reaction enthalpy and free energy for all gas phase and adsorption reactions, with the designations of the reactions from Scheme 4.1 and Scheme 4.2. 
$\Delta^{\ddagger} \mathrm{H}$

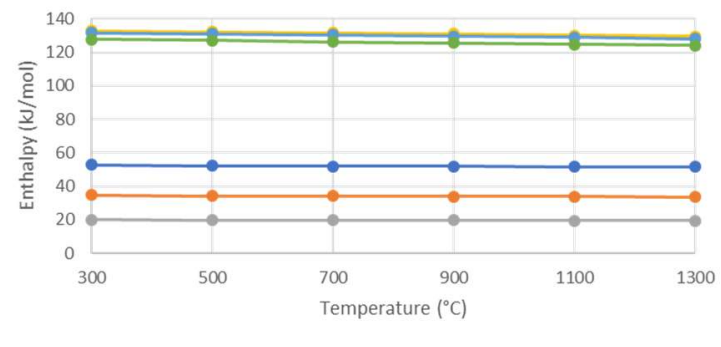

$\Delta^{\ddagger} H$

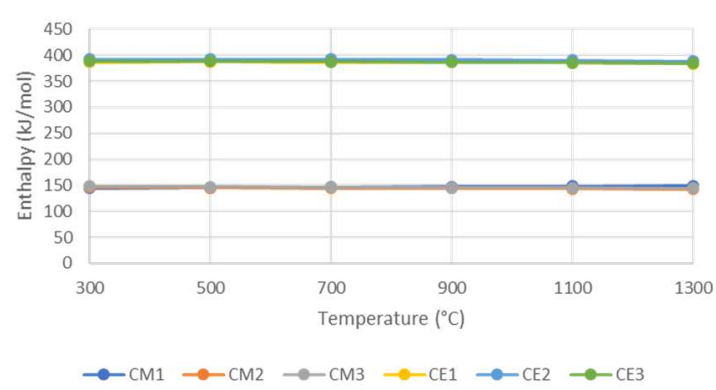

$\Delta^{\ddagger} \mathrm{G}$

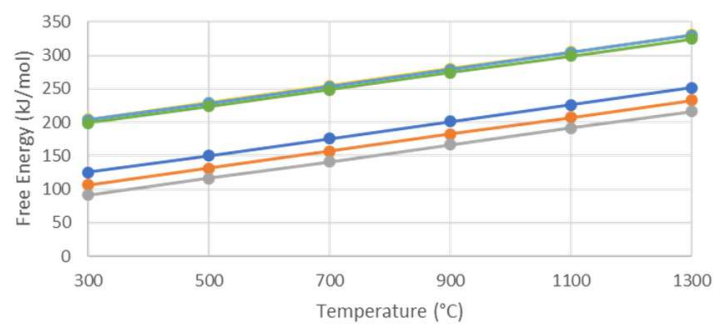

$\multimap \mathrm{BH} 1 \rightarrow \mathrm{BH} 2 \rightarrow \mathrm{BH} 3 \longrightarrow \mathrm{BC} 1 \rightarrow \mathrm{BC2} \rightarrow \mathrm{BC} 3$

$\Delta^{\ddagger} G$

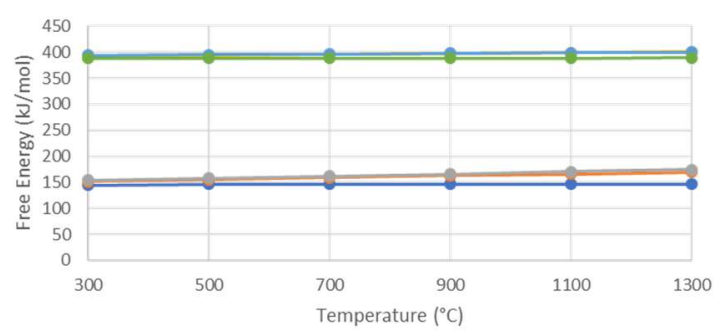

$\rightarrow$ CM1 $\bullet$ CM2 $\rightarrow$ CM3 $\rightarrow$ CE1 $\bullet-$ CE2 $\rightarrow$ CE3

Figure 4.11: Transition state enthalpy and free energy for all gas phase and adsorption reactions, with the designations of the reactions from Scheme 4.1 and Scheme 4.2 .
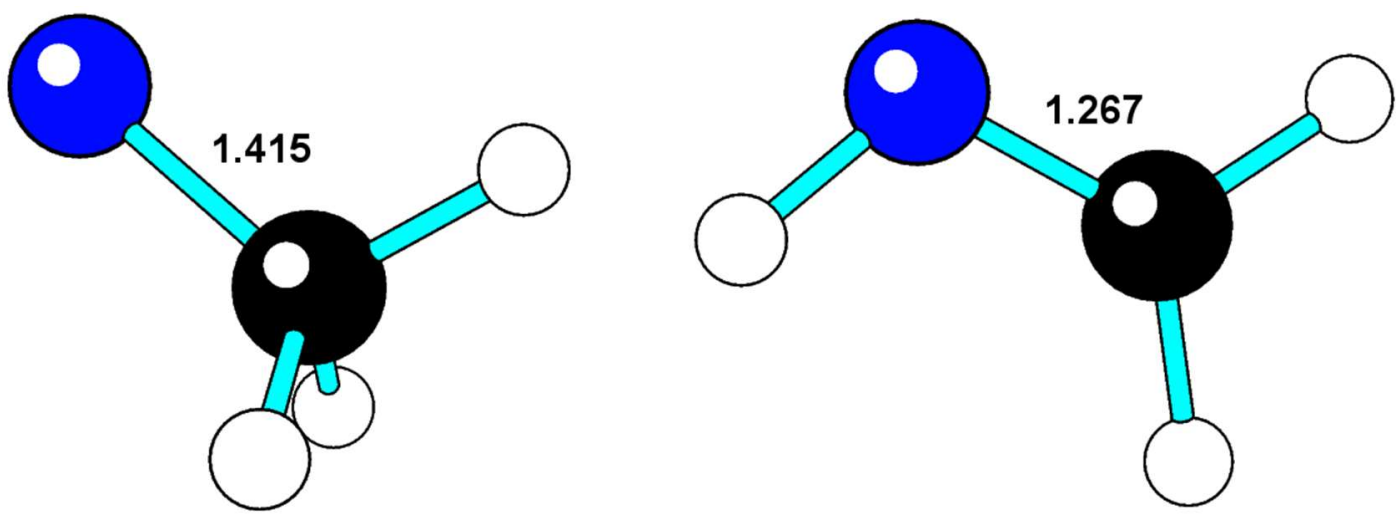

Figure 4.12: Reactant and product geometries for the rearrangement of $\mathrm{NCH}_{3}$ to $\mathrm{NHCH}_{2}$. Bond length of the carbon-nitrogen is bond given in Ångström. Blue atoms are nitrogen, black are carbon and white are hydrogen. 

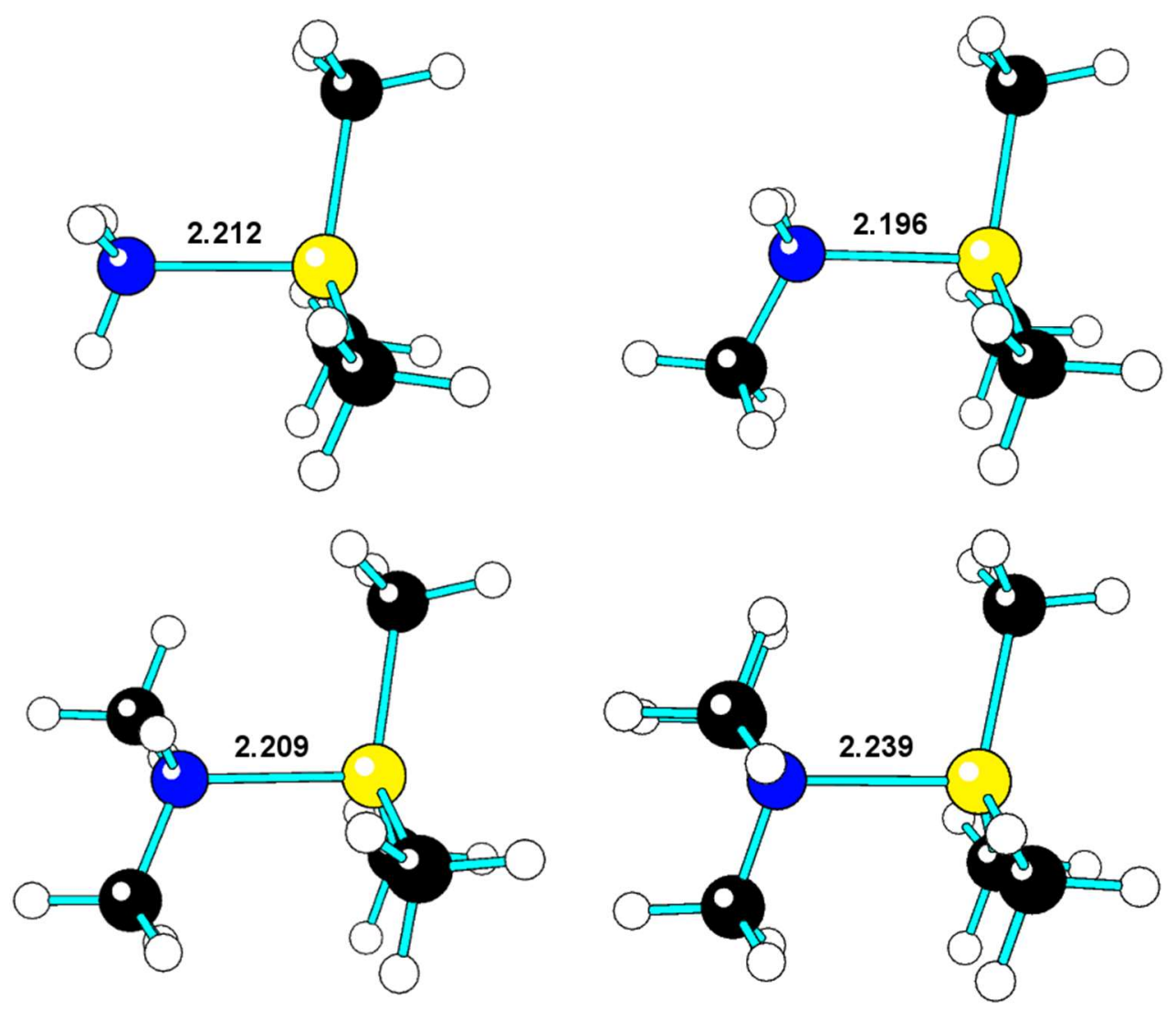

Figure 4.13: Geometry optimized structures for the Lewis adducts formed between $\mathrm{NH}_{3-\mathrm{x}}\left(\mathrm{CH}_{3}\right)_{\mathrm{x}}$ and $\mathrm{Ga}\left(\mathrm{CH}_{3}\right)_{3}$. The Ga-N distance is given in Angström. Yellow atoms are gallium, blue are nitrogen, black are carbon and white are hydrogen.

\section{Adduct formation between $\mathrm{NH}_{3-\mathrm{x}}\left(\mathrm{CH}_{3}\right)_{\mathrm{x}}$ and $\mathrm{Al}\left(\mathrm{CH}_{3}\right)_{3}$}

\section{(TMA)}

Figure 4.12 shows the TMA adduct complexes after the geometry optimization with the Al-N bond lengths indicated. The reaction energies for the adduct reactions between ammonia or the methylamines and TMA are listed in Table 4.7 at STP and Table 4.8 at CVD temperatures and pressure. As with the reaction with TMG, the adduct formation reactions with TMA are exothermic at all 
calculated temperatures, but the reaction enthalpies are less negative at higher temperatures. The reaction free energies at CVD conditions are all positive, but the forming of the adduct between dimethylamine and trimethylaluminum at $300^{\circ} \mathrm{C}$. As in the TMG case, the effect of the temperature increases the reaction free energy making the reactions less favorable at high temperatures. The adducts reaction with ammonia, methylamine and dimethylamine have similar reaction free energy and the difference is lower at higher temperatures. The reaction free energies of the adduct formation with trimethylamine is much higher than for the rest of the adduct reactions and a difference to that of the TMG case is that this reaction has the most positive reaction free energy for all CVD temperatures. The release of methane and ethane from the TMA adducts are more exothermic than the release from the TMG adducts. The reaction free energy is negative for all reaction due to them being entropic favorable. As with the TMG case the releases have higher entropy as the nitrogen gets more methyl groups and that the release of ethane is more favorable than the release of methane when both could be released.

The sum reactions for the adduct formation and release of methane and ethane are exothermic at all temperatures. All reactions but the formation of the adduct of TMA and dimethylamine and release of methane, which have a positive reaction free energy at higher temperatures, have negative reaction free energy at all temperatures. 

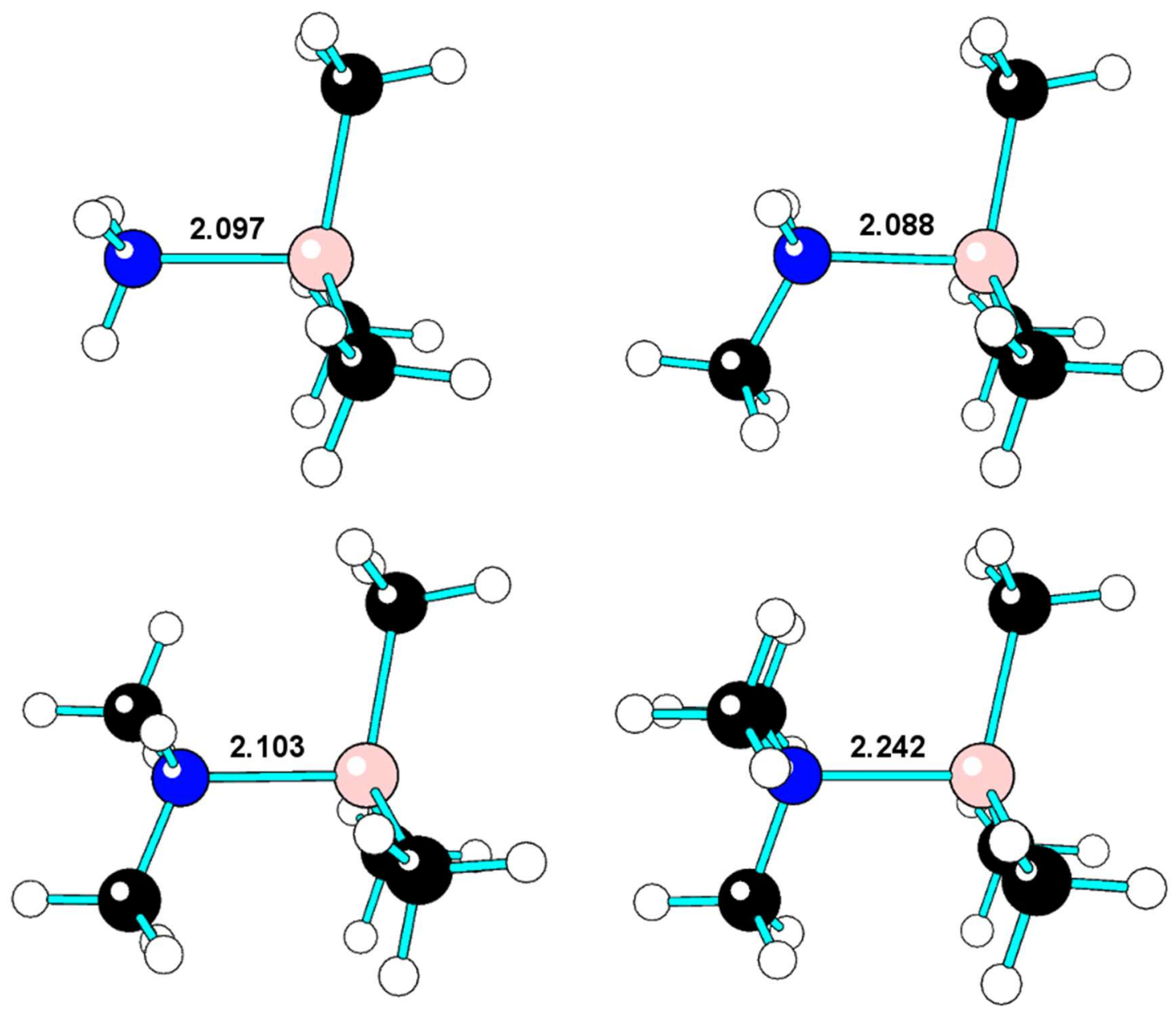

Figure 4.14: Geometry optimized structures for the Lewis adducts formed between $\mathrm{NH}_{3-\mathrm{x}}\left(\mathrm{CH}_{3}\right)_{\mathrm{x}}$ and $\mathrm{Al}\left(\mathrm{CH}_{3}\right)_{3}$. The Al-N distance is given in Angström. Salomon atoms are aluminum, blue are nitrogen, black are carbon and white are hydrogen. 
Table 4.7: Adduct formation of between $\mathrm{NH}_{3-\mathrm{x}}\left(\mathrm{CH}_{3}\right)_{\mathrm{x}}$ and $\mathrm{Al}\left(\mathrm{CH}_{3}\right)_{3}$ (TMA) and subsequent hydrocarbon elimination reactions at CVD conditions: $300^{\circ} \mathrm{C}-1300^{\circ} \mathrm{C}$ and 50 mbar. All energies are given in $\mathrm{kJ} / \mathrm{mol}$.

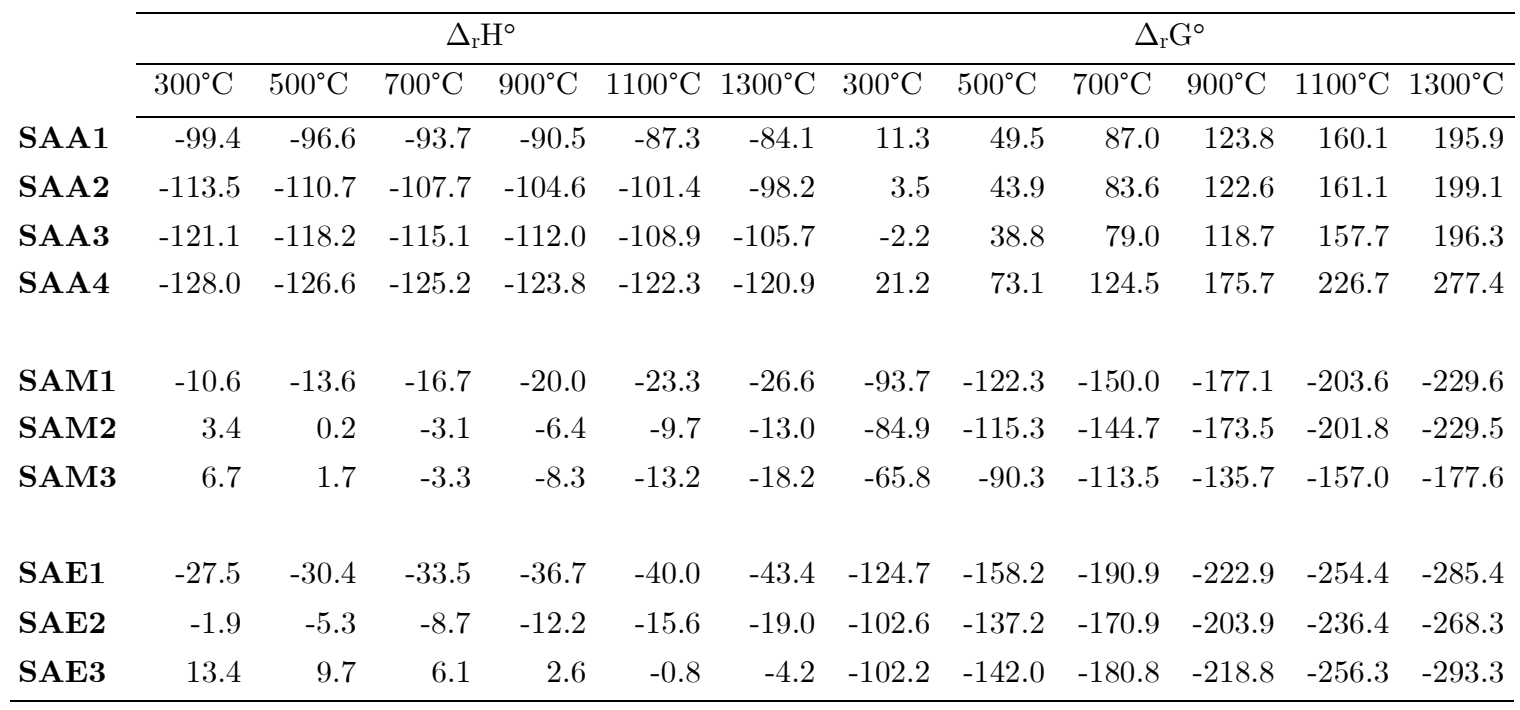

\section{Dispersion correction effects on surface interaction energies}

In Table 4.8 are the surface interaction reaction energies using the functional B3LYP but now instead without empirical dispersion listed for comparison with the values including the correction in Table 4.6. For the most reactions the difference between the two calculations with and without the dispersion is less than $50 \mathrm{~kJ} / \mathrm{mol}$ and the difference increases as the temperature is increased. The larges difference in energy was for the non-chemisorbed $\mathrm{N}\left(\mathrm{CH}_{3}\right)_{3}$. 
Table 4.8: Surface interaction of ammonia and methylamines with GaN at CVD conditions, i.e. $300^{\circ} \mathrm{C}-1300^{\circ} \mathrm{C}$ at 50 mbar, modelled using the functional B3LYP but now without empirical dispersion included. All values are given in $\mathrm{kJ} / \mathrm{mol}$.

\begin{tabular}{lrrrrrrrrrrrrr} 
& \multicolumn{1}{c}{$\Delta_{\mathrm{r}} \mathrm{H}^{\circ}$} \\
\cline { 2 - 13 } & \multicolumn{1}{c}{$300^{\circ} \mathrm{C}$} & $500^{\circ} \mathrm{C}$ & $700^{\circ} \mathrm{C}$ & $900^{\circ} \mathrm{C}$ & $1100^{\circ} \mathrm{C}$ & $1300^{\circ} \mathrm{C}$ & $300^{\circ} \mathrm{C}$ & $500^{\circ} \mathrm{C}$ & $700^{\circ} \mathrm{C}$ & $900^{\circ} \mathrm{C}$ & $1100^{\circ} \mathrm{C}$ & $1300^{\circ} \mathrm{C}$ \\
\cline { 2 - 13 } D30 & 13.7 & 14.6 & 15.3 & 15.9 & 16.5 & 17.0 & 66.1 & 84.2 & 102.2 & 119.9 & 137.6 & 155.2 \\
D31 & 16.1 & 17.1 & 17.8 & 18.4 & 18.9 & 19.4 & 86.5 & 110.9 & 135.1 & 159.2 & 183.2 & 207.0 \\
D32 & 115.4 & 118.7 & 121.4 & 123.9 & 126.1 & 128.2 & 150.7 & 162.5 & 173.5 & 184.0 & 194.1 & 203.8 \\
D33 & 111.9 & 111.7 & 111.0 & 110.0 & 108.8 & 107.5 & 175.2 & 197.3 & 219.5 & 241.9 & 264.5 & 287.2 \\
& & & & & & & & & & & & \\
D20 & -239.8 & -237.6 & -235.7 & -233.8 & -232.1 & -230.4 & -185.0 & -166.2 & -147.9 & -130.1 & -112.5 & -95.2 \\
D21 & -197.8 & -196.0 & -194.3 & -192.7 & -191.1 & -189.4 & -125.5 & -100.6 & -76.1 & -51.9 & -28.1 & -4.4 \\
D22 & -151.0 & -150.7 & -150.5 & -150.4 & -150.4 & -150.3 & -60.9 & -29.5 & 1.8 & 33.1 & 64.4 & 95.7 \\
& & & & & & & & & & & & \\
D10 & -188.1 & -188.3 & -188.7 & -189.3 & -190.0 & -190.6 & -131.5 & -111.7 & -91.9 & -71.9 & -51.9 & -31.7 \\
D11 & -144.2 & -145.0 & -146.1 & -147.4 & -148.8 & -150.2 & -67.3 & -40.3 & -13.1 & 14.4 & 42.1 & 70.0 \\
DRE & 25.6 & 29.2 & 32.1 & 34.6 & 36.9 & 39.0 & 80.8 & 99.5 & 117.3 & 134.6 & 151.4 & 168.0 \\
& & & & & & & & & & & & \\
D00 & -136.4 & -136.2 & -136.3 & -136.7 & -137.2 & -137.7 & -97.1 & -83.4 & -69.7 & -56.0 & -42.2 & -28.3 \\
\hline
\end{tabular}


Electron Image 3

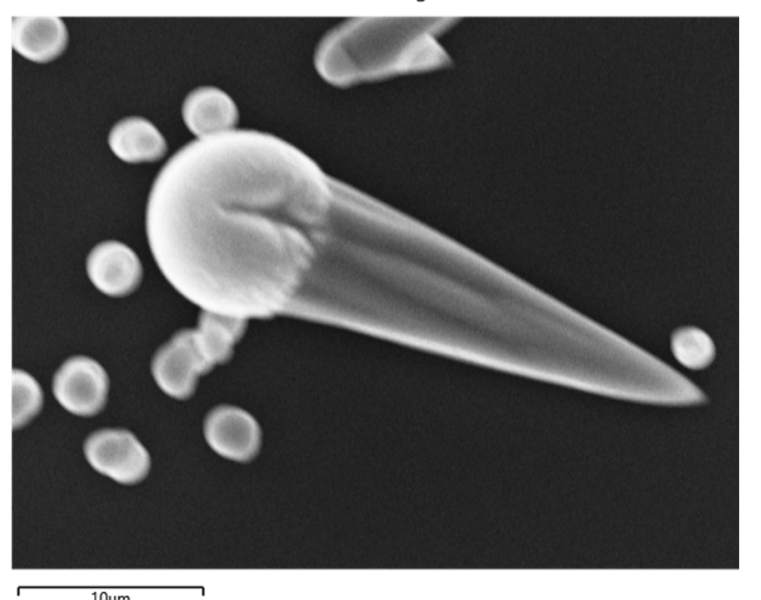

$10 \mu \mathrm{m}$

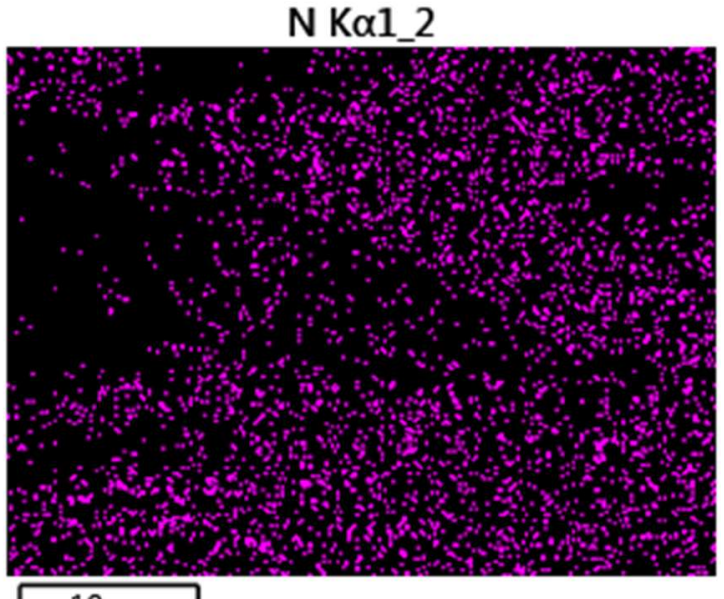

$10 \mu \mathrm{m}$

$\mathrm{O} K \alpha 1$

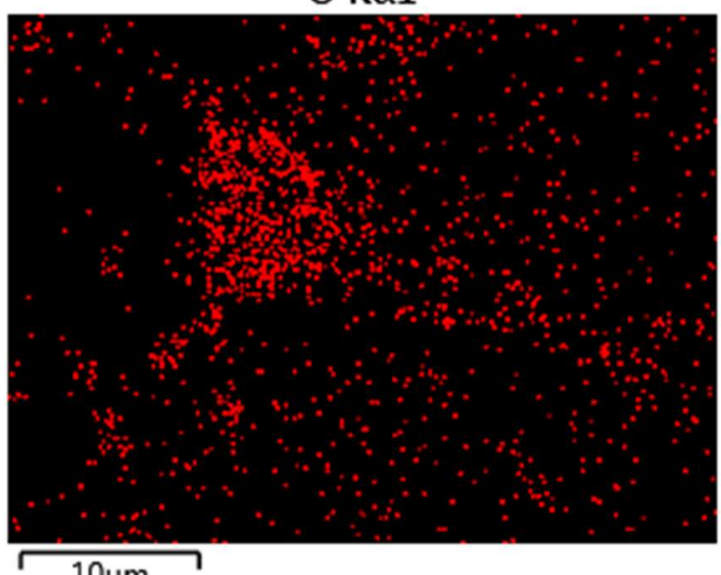

$10 \mu \mathrm{m}$
Ga K $\alpha 1$

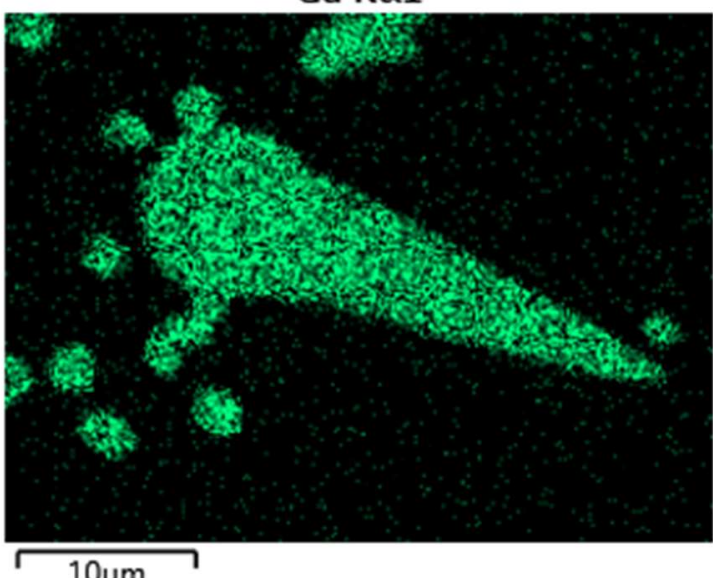

C K $\alpha 12$

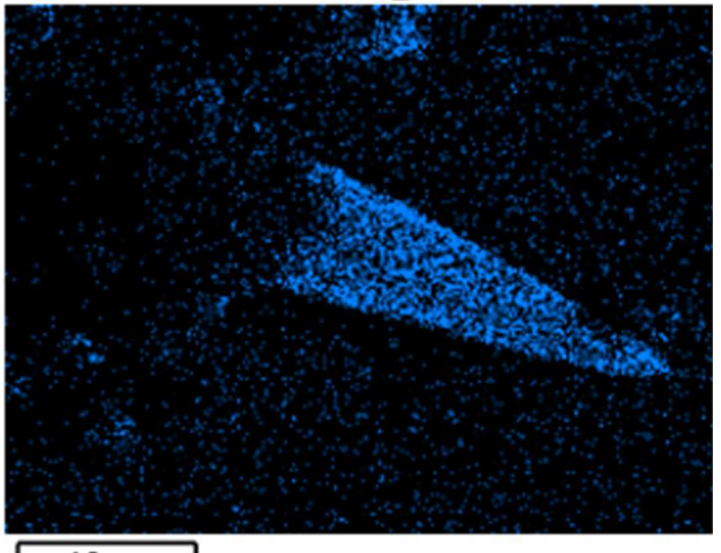

Al $K \alpha 1$

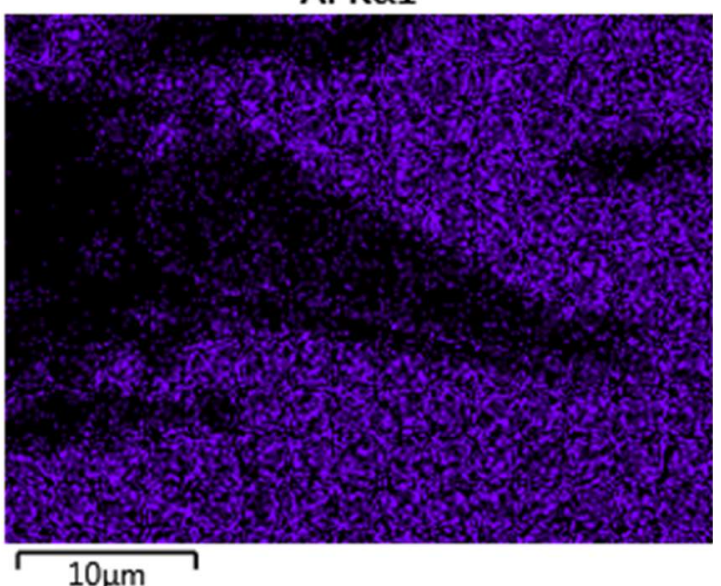

Figure 4.15: Elemental mapping by EDX of a droplet with a cone from a CVD samples grown at $925{ }^{\circ} \mathrm{C}$ from $\mathrm{Ga}\left(\mathrm{CH}_{3}\right)_{3}$ and $\mathrm{N}\left(\mathrm{CH}_{3}\right)_{3}$ using $\mathrm{N} / \mathrm{Ga}=570$. 


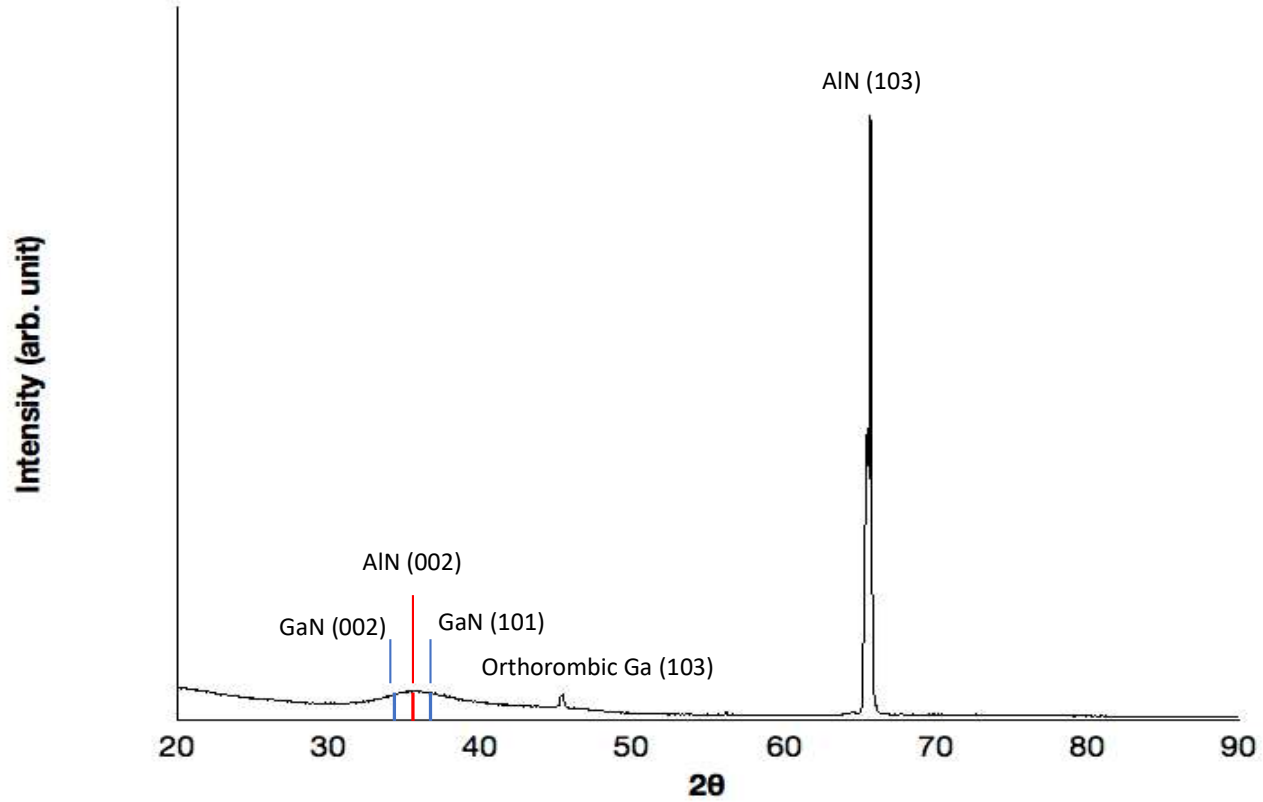

Figure 4.16: GIXRD of a CVD sample grown at $925{ }^{\circ} \mathrm{C}$ from $\mathrm{Ga}\left(\mathrm{CH}_{3}\right)_{3}$ and $\mathrm{N}\left(\mathrm{CH}_{3}\right)_{3}$ using $\mathrm{N} / \mathrm{Ga}=570$. 


\title{
Chapter 5 - Part I
}

\section{In-situ Activation of an Indium(III) Triazenide}

\author{
Precursor for Epitaxial Growth of Indium
}

\section{Nitride by Atomic Layer Deposition}

Modified from the original manuscript published as:

O'Brien, N. J.; Rouf, P.; Samii, R.; Ronnby, K.; Buttera, S. C.; Hsu, C.-W.; Ivanov,

I. G.; Kessler, V.; Ojamäe, L.; Pedersen, H. In-Situ Activation of an Indium(III)

Triazenide Precursor for Epitaxial Growth of Indium Nitride by Atomic Layer

Deposition. Chem. Mater. 2020. https://doi.org/10.1021/acs.chemmater.9b05171. 


\subsubsection{Abstract}

Indium nitride $(\mathrm{InN})$ is characterized by its high electron mobility, making it a ground-breaking material for high frequency electronics. The difficulty of depositing high-quality crystalline $\mathrm{InN}$ currently impedes its broad implementation in electronic devices. Herein, we report a new highly volatile $\operatorname{In}(\mathrm{III})$ triazenide precursor and demonstrate its ability to deposit high-quality epitaxial hexagonal InN by atomic layer deposition (ALD). The new In(III) precursor, the first example of a homoleptic triazenide used in a vapor deposition process, was easily synthesized and purified by sublimation. Thermogravimetric analysis showed single step volatilization with an onset temperature of $145{ }^{\circ} \mathrm{C}$ and negligible residual mass. Strikingly, two temperature intervals with self- limiting growth were observed when depositing InN films. In the high-temperature interval, the precursor underwent a gas-phase thermal decomposition inside the ALD reaction chamber to produce a more reactive $\operatorname{In}(\mathrm{III})$ compound while retaining self-limiting growth behavior. Density functional theory calculations revealed a unique two-step decomposition process, which liberates three molecules of each propene and $\mathrm{N}_{2}$ to give a smaller tricoordinated $\operatorname{In}(\mathrm{III})$ species. Stoichiometric InN films with very low levels of impurities were grown epitaxially on $4 \mathrm{H}-\mathrm{SiC}$. The InN films deposited at $325{ }^{\circ} \mathrm{C}$ had a sheet resistivity of $920 \Omega /$ sq. This new triazenide precursor enables ALD of 
InN for semiconductor applications and provides a new family of $\mathrm{M}-\mathrm{N}$ bonded precursors for future deposition processes.

\subsubsection{Introduction}

The high electron mobility of indium nitride $(\operatorname{InN})^{71,176}$ makes it a very interesting material for the conduction channel in high electron mobility transistors (HEMTs) and high frequency electronics. InN can be integrated in state-of-the-art electronic devices that are based on aluminum- and gallium nitride (AlN \& GaN) and their alloys, given the close similarities in their crystal lattices. The implementation of InN into device structures requires deposition of epitaxially orientated and stoichiometric InN thin films with negligible impurities on substrates with high thermal conductivity, such as SiC. ${ }^{177}$ The current chemical vapor deposition (CVD) processes used for depositing thin films of AlN and GaN, at high temperatures of 800-1000 ${ }^{\circ} \mathrm{C}$, are not suitable to depositing InN as it decomposes to In metal and $\mathrm{N} 2$ gas at approximately $500{ }^{\circ} \mathrm{C} .{ }^{178}$ This sets a strict upper temperature limit for $\mathrm{CV} D$ of $\mathrm{InN}$ where the reactivity of the nitrogen precursor, ammonia $\left(\mathrm{NH}_{3}\right)$, is very low, forcing $\mathrm{N} / \mathrm{In}$ ratios in the order of $10^{5}$ and thus poorly functioning CVD chemistry. ${ }^{179,180}$ Atomic layer deposition (ALD) is a low temperature time-resolved form of CVD, in which the metal and non-metal precursors are pulsed into the reaction chamber sequentially. This process allows for the deposition of the resulting film to be solely

governed by surface chemical reactions. ${ }^{181}$ The ALD cycle is repeated hundreds of 
times to deposit films with controlled thickness, excellent large-area uniformity, and conformity. We envision that ALD is the way forward to realize electronics based on InN. Although ALD is routinely used in the production of modern electronic devices, its potential for $\mathrm{InN}$ is yet to be explored. This is mostly due to the poor deposition chemistry afforded at low temperatures by the commonly used trimethylindium $\left(\mathrm{InMe}_{3}\right)$ precursor. ALD of $\mathrm{InN}$ from $\mathrm{InMe}_{3}$ in combination with $\mathrm{N}_{2}{ }^{182,183}$ and $\mathrm{NH}_{3}{ }^{184}$ plasma has produced epitaxial $\mathrm{InN}$ on $\mathrm{GaN}(0001), \mathrm{Si}(111)$, and sapphire substrates, ${ }^{182,185,186}$ but renders films with high carbon and oxygen impurity levels and non-stoichiometric $\mathrm{In} / \mathrm{N}$ ratios.

For successful ALD, it is important to have a metal precursor with high volatility and thermal stability that cleanly reacts with the surface to avoid unwanted byproducts. ${ }^{181}$ Efforts have been made to develop In(III) precursors with favorable surface chemistry for ALD of InN by replacing the $\mathrm{M}-\mathrm{C}$ bonds of $\mathrm{InMe}_{3}$ with $\mathrm{M}-\mathrm{N}$ bonds. Homoleptic tricoordinated $\mathrm{M}-\mathrm{N}$ bonded compounds have been reported $^{187-191}$, but these often require bulky ligands to stabilize the In center, which reduces their volatility. Instead, homoleptic hexacoordinated $\mathrm{M}-\mathrm{N}$ bonded precursors, $\operatorname{In}(\mathrm{III})$ tris-amidinate $\left(\operatorname{In}(\mathrm{amd}){ }_{3}\right)^{192}$, formamidinate $\left(\operatorname{In}(\text { famd })_{3}\right)^{193}$ and guanidinate $\left(\operatorname{In}(\text { guan })_{3}\right)^{194,195}$ precursors, have shown increased thermal stability in comparison to tricoordinated $\operatorname{In}(\mathrm{III})$ precursors and were first used for ALD of $\mathrm{In}_{2} \mathrm{O}_{3}{ }^{193,195}$ and $\operatorname{In}_{2} \mathrm{~S}_{3}{ }^{196}$ Recently, we investigated these precursors with $\mathrm{NH}_{3}$ 
plasma for ALD of crystalline InN. ${ }^{197}$ The best results were achieved with $\operatorname{In}(\text { famd })_{3}$, giving the highest degree of crystallinity, lowest impurity levels and $\mathrm{In} / \mathrm{N}$ ratio of 1.01. It was further revealed that smaller and less electron donating substituents on the endocyclic carbon of the amidinate ligand backbone led to an improved surface chemistry in ALD of InN films. To further explore this trend, we envisaged changing the endocyclic position to an even smaller and more electronwithdrawing moiety. A ligand that is closely related to the amidinates is the triazenide, which differs only by the electronegative nitrogen atom in the endocyclic position of the ligand backbone. Although the binding modes of amidinates are similar to those of triazenides, the extra nitrogen atom is thought to make the chelating nitrogens weaker binders. ${ }^{198,199}$ This would benefit the surface reactions of the precursor during deposition by making the In-N bonds weaker and the In metal center more electrophilic. There are many examples of homoleptic triazenide complexes in the literature ${ }^{199-208}$, including an $\operatorname{In}(\mathrm{III})$ complex $(\operatorname{In}(\text { dptriaz}))^{209,210}$, however these are undesirable for vapor deposition due to the 1,3diphenyltriazenide ligand. Homoleptic 1,3-dimethyltriazenide ${ }^{211}$ and heteroleptic 1tert-butyl-3-alkyltriazenide ${ }^{212,213}$ complexes have also been reported for various metals, but these suffer from low volatility and thermal stability. To the best of our knowledge, there are no examples of a homoleptic 1,3-dialkyltriazenide complex used for vapor deposition. Herein, we report the synthesis, structure and physical properties of a new $\operatorname{In}(\mathrm{III})$ precursor, tris(1,3-diisopropyltriazenide)indium(III) $\mathbf{5}$, 
and demonstrate it as an excellent ALD precursor for epitaxial InN thin films on 4H-SiC.

\subsubsection{Experimental}

\section{General Experimental Procedures}

Caution! As catenated nitrogen compounds are known to be associated with explosive hazards, isopropylazide and compound $\mathbf{5}$ are possible explosive energetic materials. Although we have not experienced any problems in the synthesis, characterization, sublimation and handling of compound 5, its energetic properties have not been investigated and are therefore unknown. We therefore highly recommend all appropriate standard safety precautions for handling explosive materials (safety glasses, face shield, blast shield, leather gloves, polymer apron and ear protection) be used at all times when working with compound $\mathbf{5}$ and isopropylazide. All reactions and manipulations were carried out under an $\mathrm{N}_{2}$ atmosphere on a Schlenk line using Schlenk air-free techniques and in a GloveboxSystemtechnik dry box. All anhydrous solvents were purchased from SigmaAldrich ${ }^{\mathrm{TM}}$ and further dried with $4 \AA$ molecular sieves. $\mathrm{InCl}_{3} \quad(98 \%)$ and isopropyllithium solution were purchased from Sigma-Aldrich and both used

without further purification. Isopropylazide was synthesized according to the literature procedure. ${ }^{214}$ All NMR spectra were measured with an Oxford Varian 
$300 \mathrm{MHz}$ spectrometer. The $\mathrm{C}_{6} \mathrm{D}_{6}$ solvent peaks were used as an internal standard for the ${ }^{1} \mathrm{H}$ NMR $(300 \mathrm{MHz})$ and ${ }^{13} \mathrm{C}\left\{{ }^{1} \mathrm{H}\right\}$ NMR $(75 \mathrm{MHz})$ spectra. The electrospray ionization (ESI)-mass spectrum data was obtained on a Thermo Scientific LCQ Fleet ESI-MS instrument. The melting point was determined in a capillary sealed under $\mathrm{N}_{2}$ with a Stuart ${ }^{\circledR}$ SMP10 melting point apparatus and is uncorrected. Elemental analysis was performed by Mikroanalytisches Laboratorium Kolbe, Germany.

\section{Synthesis of tris(1,3-diisopropyltriazenide)indium(III) (5)}

To a solution of isopropylazide ${ }^{214}(5.90 \mathrm{~g}, 69.3 \mathrm{mmol})$ in $\mathrm{Et}_{2} \mathrm{O}(100 \mathrm{~mL})$ at $-78{ }^{\circ} \mathrm{C}$ was added isopropyllithium (0.7 M in pentane, $99.0 \mathrm{~mL}, 69.3 \mathrm{mmol})$. The reaction mixture was stirred for $30 \mathrm{~min}$ and then allowed to warm up to room temperature for $1 \mathrm{~h}$. This solution was then added to a $-78{ }^{\circ} \mathrm{C}$ solution of $\mathrm{InCl}_{3}(5.11 \mathrm{~g}, 23.1$ mmol) in THF (100 mL) via cannula and the mixture was stirred at this temperature for $20 \mathrm{~min}$. The reaction was warmed to room temperature and stirred for $16 \mathrm{~h}$. The reaction mixture was concentrated under reduced pressure and the resulting residue was dissolved in $n$-hexanes, filtered through a pad of Celite and concentrated under reduced pressure to give a light-yellow solid. The solid was purified by sublimation at $80{ }^{\circ} \mathrm{C}(0.5$ mbar $)$ to give compound $\mathbf{5}$ as a solid $(8.19 \mathrm{~g}$, $71 \%)$. 
5: Colorless solid, decomp. $212-214{ }^{\circ} \mathrm{C} .{ }^{1} \mathrm{H}$ NMR $\left(300 \mathrm{MHz}, \mathrm{C}_{6} \mathrm{D}_{6}\right) \delta 1.23(\mathrm{~d}, J=$ $\left.6.0 \mathrm{~Hz}, 36 \mathrm{H}, \mathrm{CH}_{3}\right), 4.06$ (sept, $\left.J=6.0 \mathrm{~Hz}, 6 \mathrm{H}, \mathrm{CH}\right) .{ }^{13} \mathrm{C}\left\{{ }^{1} \mathrm{H}\right\} \mathrm{NMR}\left(75 \mathrm{MHz}, \mathrm{C}_{6} \mathrm{D}_{6}\right)$ $\delta 24.0\left(\mathrm{~s}, \mathrm{CH}_{3}\right), 53.7(\mathrm{~s}, \mathrm{CH})$. LRMS (ESI, positive) $m / z=500[\mathrm{M}+\mathrm{H}]^{+}$. Anal. calcd for $\mathrm{C}_{18} \mathrm{H}_{42} \mathrm{InN}_{9}$ : C, $43.29 \%$; H, 8.48\%; N, 25.24\%. Found: C, 42.64\%; H, 8.25\%; N, $24.90 \%$.

\section{X-ray Crystallographic Analysis}

Colorless single crystals were obtained by recrystallization from $n$-hexanes at -35 ${ }^{\circ} \mathrm{C}$ for $\mathbf{5}$. The single crystals were used for X-ray diffraction data collection on a Bruker D8 SMART Apex-II diffractometer, using graphite-monochromated Mo-Ka radiation $(\lambda=0.71073 \AA)$. All data was collected in hemisphere with over $95 \%$ completeness to $2 \theta<50.05^{\circ}$. The structures were solved by direct methods. The coordinates of metal atoms were determined from the initial solutions, and of the $\mathrm{N}$ and $\mathrm{C}$ methods, located in subsequent differential Fourier syntheses. All nonhydrogen atoms were refined first in isotropic and then in anisotropic approximation using Bruker SHELXTL software. Additional crystal data is available at the Cambridge Crystallographic Data Centre, deposition no. CCDC 1966432.

\section{Thermogravimetric Analysis}

Volatilization and vapor pressure curves were collected using a TA Instruments thermogravimetric analysis Q500 tool inside an $\mathrm{N}_{2}$ filled glovebox. The ramp 
experiment of $\mathbf{5}$ was undertaken in tared platinum pans loaded with $6 \mathrm{mg}$ and 17 $\mathrm{mg}$ of $\mathbf{5}$ for low and high mass volatilization experiments, respectively. The furnace was heated at a rate of $10{ }^{\circ} \mathrm{C} / \mathrm{min}$ to $500{ }^{\circ} \mathrm{C}$ with a maintained nitrogen flow rate of $60 \mathrm{sccm}$.

\section{Differential Scanning Calorimetry Analysis}

The differential scanning calorimetry (DSC) measurement for $\mathbf{5}$ was performed on a TA Instruments DSC Q10 tool. A sample of $\sim 0.2 \mathrm{mg}$ was prepared in a sealed aluminum pan in an $\mathrm{N}_{2}$ filled glovebox. The sample of $\mathbf{5}$ and a blank reference pan were heated at a rate of $10{ }^{\circ} \mathrm{C} / \min$ to $400{ }^{\circ} \mathrm{C}$.

\section{Quantum-Chemical Computations}

All quantum chemical computations were preformed using Gaussian 16 software. ${ }^{215}$ Structural optimization and harmonic normal mode vibrational calculations were done using the hybrid DFT method B3LYP216,217 together with Grimme's version 3 dispersion correction ${ }^{218}$ and def2TZVP ${ }^{219,220}$ basis set. The decomposition path was investigated by searching for possible stable structures as well as finding transition states connecting these structures. Minima were confirmed to have no imaginary frequencies while transition states were verified to have one imaginary frequency, lying along the reaction path. 


\section{Film Deposition}

A hot-wall Picosun R-200 was used for the deposition of InN, which is equipped with a Litmas remote plasma source. The tool operated at 4 mbar with a continuous flow of high purity $\mathrm{N}_{2}(99,999 \%)$ into the chamber, which was also used as the purge gas. The system was equipped with a traditional stainless-steel precursor container (bubbler), albeit without dip-tube for the incoming carrier gas. The system was baked at $450{ }^{\circ} \mathrm{C}$ for 2 hours with a $300 \mathrm{sccm}$ flow of $\mathrm{N}_{2}$ to remove traces of $\mathrm{H}_{2} \mathrm{O}$ and $\mathrm{O}_{2}$ in the deposition chamber after exposure to the atmosphere during substrate exchange. In an $\mathrm{N}_{2}$ filled glovebox, $\sim 300 \mathrm{mg}$ of the precursor was weighed into a glass vial, which was placed in a stainless-steel container and then assembled onto the ALD tool. Caution! The bubbler must not be overfilled with multigram scale of precursor 5 due to possible explosion risk. The optimal temperature for the bubbler in order to obtain high enough vapor pressure to saturate the substrate surface and obtain optimal growth was $125{ }^{\circ} \mathrm{C}$. An amount of $\sim 300 \mathrm{mg}$ of precursor was enough for 500 cycles, and increased linearly with the number of cycles. $\mathrm{A} \mathrm{NH} 3$ plasma was used as the nitrogen source, containing a $100 / 75 \mathrm{sccm} \mathrm{Ar} / \mathrm{NH}_{3}$ gas mixture with a $2800 \mathrm{~W}$ plasma power and was located approximately $75 \mathrm{~cm}$ above the substrate. ${ }^{184}$ Unless otherwise stated, a $9 \mathrm{~s} \mathrm{NH}_{3}$ pulse was used followed by a $10 \mathrm{~s}$ purge. The $\mathrm{Si}(100)$ and $4 \mathrm{H}-\mathrm{SiC}$ substrates were cut into $15 \times 15 \mathrm{~mm}^{2}$ pieces and films were deposited on them without any further 
ex-situ cleaning. Prior to deposition, a $120 \mathrm{~s}$ of $\mathrm{H}_{2}$ plasma (1000 W, gas mixture of $\left.\mathrm{Ar} / \mathrm{H}_{2} 100 / 10 \mathrm{sccm}\right)$ and $\mathrm{N}_{2}$ plasma $\left(2800 \mathrm{~W}\right.$, gas mixture of $\left.\mathrm{Ar} / \mathrm{N}_{2} 80 / 380 \mathrm{sccm}\right)$ was employed to nitridize the substrate surface and to remove residual $\mathrm{H} 2 \mathrm{O}$ and O2 from inside the deposition chamber.

\section{Film Characterization}

Film thickness and film crystallinity $(\theta-2 \theta)$ were measured with X-ray Reflectivity (XRR), PANalytical X'Pert PRO with a Cu-anode tube and Bragg-Brentano HD optics. To analyze the thickness, the software PANalytical X'Pert reflectivity and a two-layer model was used to fit the data, InN/substrate. PANalytical EMPYREAN MRD XRD with a Cu-anode X-ray tube and 5-axis (x-y-z-v-u) sample stage operating at $45 \mathrm{kv}$ and $40 \mathrm{~mA}$ was used in Grazing Incidence X-ray diffraction (GIXRD) mode with $0.5^{\circ}$ incident angle to analyze the crystallinity of the films. The pole figures were obtained with the same XRD equipment and operating parameters using an X-ray lens and parallel plate collimator. The morphology of the films was examined with a LEO 1550 Scanning electron microscopy (SEM) operating at an acceleration energy of $10 \mathrm{kV}$. Kratos AXIS Ultra DLD X-ray photoelectron spectroscopy (XPS) was used to analyze the composition and the chemical environment of the atoms in the film. The XPS tool was equipped with an Ar sputtering source $(0.5 \mathrm{keV})$, which was used for clean sputtering and depth profiling of the films. To analyze the data CasaXPS was used, the high- 
resolution scans were fitted by Gaussian-Laurentius functions and Shirley background. Raman spectra were recorded on a micro-Raman setup utilizing 100X objective and 532-nm solid-state laser (Coherent, Sapphire SF 532-150). The laser was focused to $\sim 0.8 \mu \mathrm{m}$ spot on the sample and a power below $1 \mathrm{~mW}$ was used to avoid overheating of the sample. The Raman signal was dispersed by a monochromator (Jobin Yvon, HR460) coupled to a CCD camera. With the 600 grooves/mm grating used in this measurement the resolution was about $5.5 \mathrm{~cm}^{-1}$. To obtain high signal-to-noise ratio 100 subsequent acquisitions by $10 \mathrm{~s}$ were acquired for each sample resulting in a total acquisition time of $1000 \mathrm{~s}$. No changes were observed in the spectra during the series, indicating that the laser power utilized was sufficiently low to avoid thermal damage.

\subsubsection{Results and Discussion}

\section{Synthesis and Characterization of In(III) Triazenide}

\section{Precursor}

Reaction of isopropylazide ${ }^{214}$ with isopropyllithium generated the lithium 1,3-diisopropyltriazenide intermediate, which was reacted directly with indium trichloride $\left(\mathrm{InCl}_{3}\right)$ at $-78{ }^{\circ} \mathrm{C}$ to give tris(1,3-diisopropyltriazenide)indium(III) $\mathbf{5}$ in a $71 \%$ yield (Scheme 5.1). Compound $\mathbf{5}$ was isolated as a colorless solid after 
purification by sublimation. It was found to be highly stable at room temperature in both solid and solution state under an inert atmosphere, however exposure to air led to decomposition to a white insoluble powder thought to be of $\operatorname{In}_{2} \mathrm{O}_{3}$. The compound was fully characterized by nuclear magnetic resonance (NMR) spectroscopy, mass spectrometry (MS), elemental analysis and X-ray crystallography.

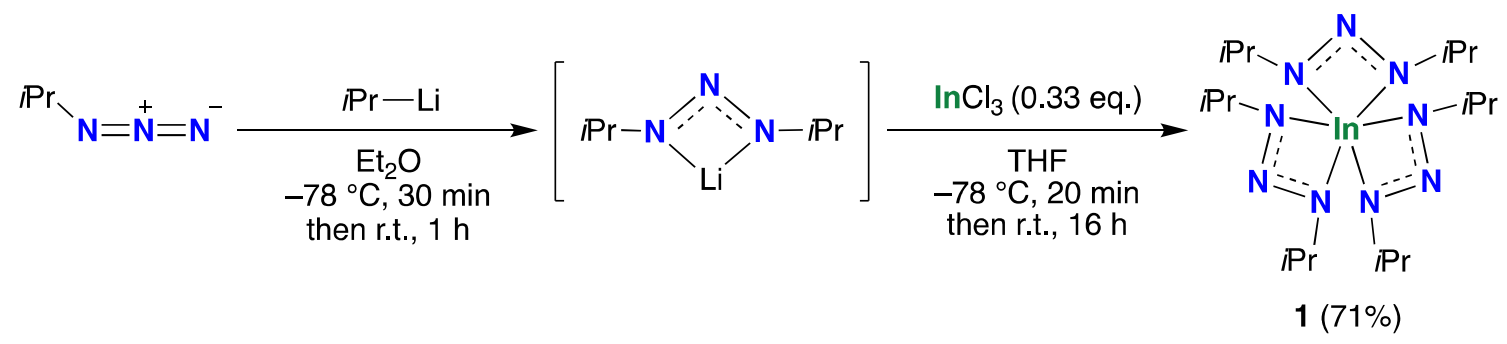

Scheme 5.1: Synthesis of tris(1,3-diisopropyltriazenide)indium(III) (5).

The crystal structure of $\mathbf{5}$ (Figure 5.1, Table 5.1-5.5) showed the In atom in a distorted-octahedral coordination geometry with the triazenide ligands in a pseudo- $C_{3}$ propeller arrangement similarly to $\operatorname{In}(\text { dptriaz })_{3}{ }^{210}$ Two of the ligands were found to be distorted over four positions and the third over two positions. The In-N bond lengths (av. 2.21(5) $\AA$ ) were similar to that of $\operatorname{In}($ dptriaz) 3 (av. $2.237(6) \AA)^{210}, \operatorname{In}(\text { famd })_{3}(\text { av. } 2.252(13) \AA)^{193}, \operatorname{In}(\text { amd })_{3}(\text { av. } 2.236(1) \AA)^{192}$, and In(guan) 3 (av. 2.260(4) $\AA)^{194,195}$, showing the alkyl-triazenide ligand has little effect on the $\mathrm{In}-\mathrm{N}$ bond distances. 


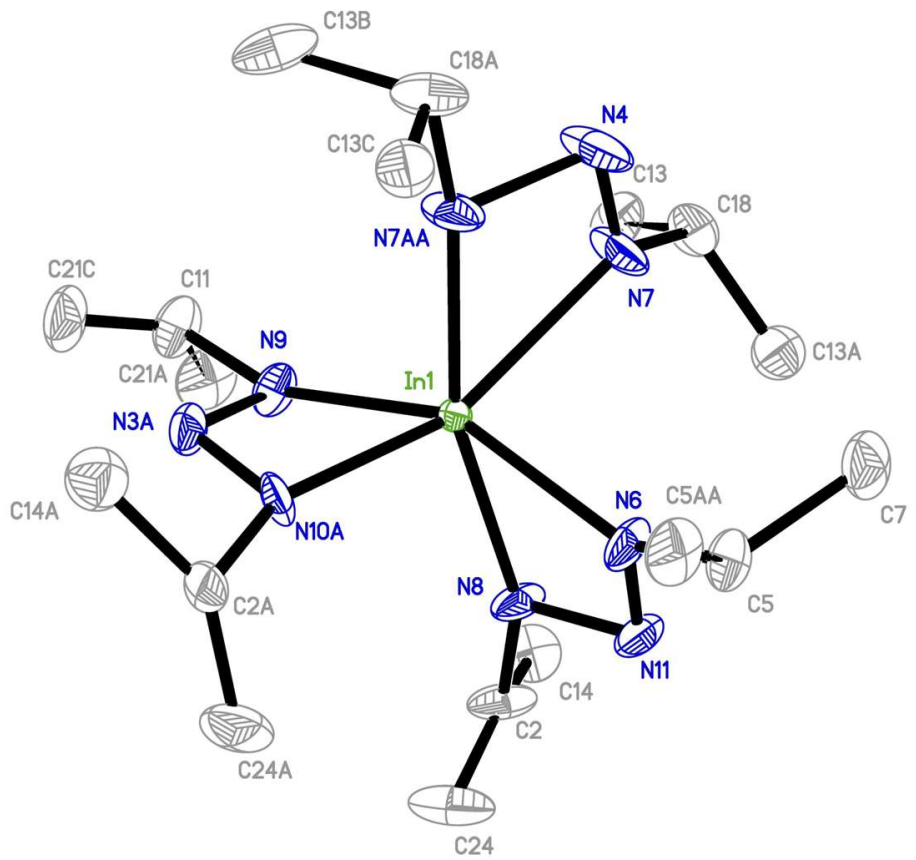

Figure 5.1: ORTEP drawing of $\mathbf{5}$ with thermal ellipsoids at the $50 \%$ probability level. All hydrogen atoms were removed for clarity.

The ${ }^{1} \mathrm{H}$ NMR spectrum of 5 in $\mathrm{C}_{6} \mathrm{D}_{6}$ showed one septet at $4.06 \mathrm{ppm}$ and one doublet at $1.23 \mathrm{ppm}$ for the methine proton and methyl groups of the isopropyl moiety, respectively (Figure 5.7). This indicates that $\mathbf{5}$ exists as a monomer in solution state, which is also reflected in the solid-state structure. MS analysis gave a molecular ion at $500 \mathrm{~m} / \mathrm{z}$, confirming its identity. Compound $\mathbf{5}$ was found to be highly volatile and sublimed at $80{ }^{\circ} \mathrm{C}$ (0.5 mbar) with no visible solid remaining. Thermogravimetric analysis (TGA) showed the new precursor volatilized completely in a single step from 145 to $215{ }^{\circ} \mathrm{C}$, with only $2 \%$ of residual mass (Figure 5.2). A thermal stress test with a higher mass loading of precursor showed a slight shift to a higher temperature $\left(160-235{ }^{\circ} \mathrm{C}\right)$ without signs of decomposition 
(Figure 5.9a). The 1 Torr vapor pressure of $\mathbf{5}$ was shown to occur at $134{ }^{\circ} \mathrm{C}$ (Figure $5.9 \mathrm{~b})$ and its $\Delta \mathrm{H}$ of vaporization was $65.8 \mathrm{~kJ} \mathrm{~mol}^{-1}$ (Figure $5.9 \mathrm{c}$ ).

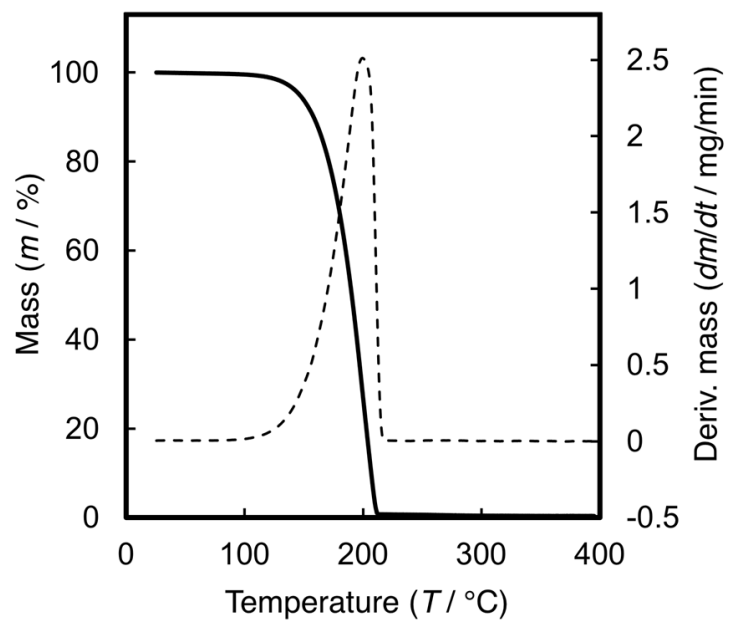

Figure 5.2: Thermogravimetric analysis of compound $\mathbf{5}$.

The heterolytic bond dissociation energy of the $\mathrm{In}-\mathrm{N}$ bonds for $\mathbf{5}$ was calculated by density functional theory (DFT) to be $289 \mathrm{~kJ} \mathrm{~mol}^{-1}$, which is lower than that of $\operatorname{In}(\text { famd })_{3}\left(312 \mathrm{~kJ} \mathrm{~mol}^{-1}\right)$ (Table 5.6) and indicates a slightly weaker In-N bond. The natural charges of the In $(+1.47)$ and $\mathrm{N}(-0.44)$ atoms of 5 show a less polarized In-N bond character in comparison to the previously used $\operatorname{In}(\text { famd })_{3}(\mathrm{In},+1.78 ; \mathrm{N},-0.69)$ (Table 5.7). Both the HOMO $\pi$ and LUMO $\pi^{*}$ frontier orbitals of $\mathbf{1}$ are localized on the $\mathrm{N}_{3}$ moiety of the triazenide ligand (Figure 5.3a and 5.3b). The high electron density on the backbone of the ligand could explain the increased volatility of $\mathbf{5}$ due to electrostatic repulsion forces in solid state. This is consistent with the crystal structure, which is highly disordered because of a lack of intermolecular interactions. 

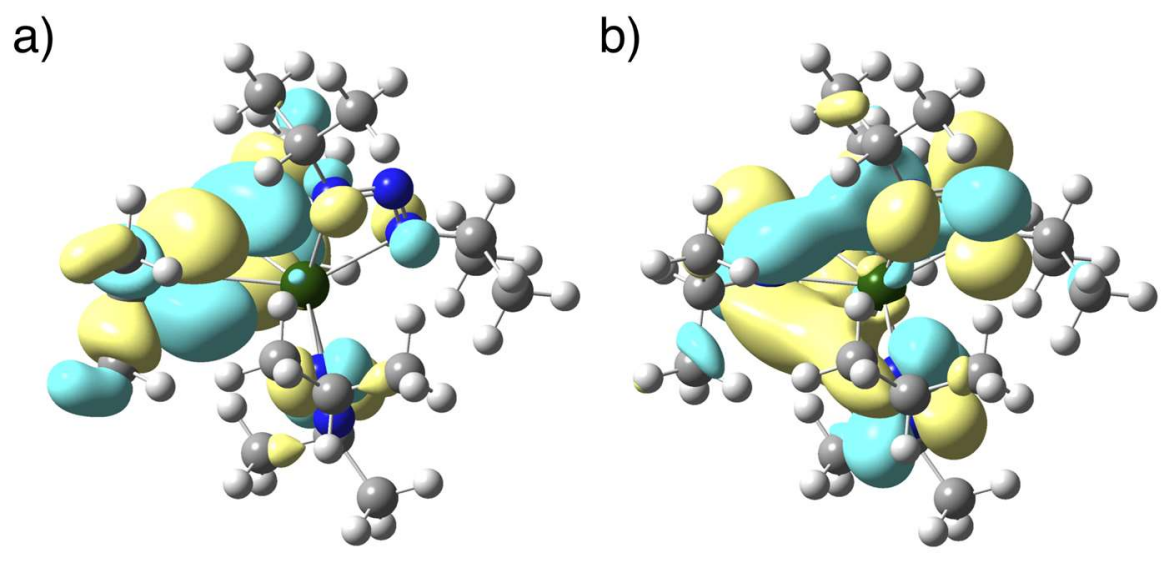

Figure 5.3: a) The HOMO $(-5.90 \mathrm{eV})$ and b) LUMO $(-0.69 \mathrm{eV})$ of $\mathbf{5}$ from DFT calculations.

\section{ALD of InN using In(III) triazenide precursor}

ALD of InN was tested initially on $\mathrm{Si}(100)$ using 5 s pulse of 5 and $9 \mathrm{~s} \mathrm{NH}_{3}$ plasma pulse, separated by a $9 \mathrm{~s} \mathrm{~N}_{2}$ purge, while varying the deposition temperature. These experiments revealed two temperature intervals where the growth per cycle (GPC) was constant with temperature, at $220-250{ }^{\circ} \mathrm{C}$ and $300-350{ }^{\circ} \mathrm{C}$ (Figure 5.4a). Saturation curves on $\mathrm{Si}(100)$ of the lower and higher temperature intervals showed saturation growth behavior with a GPC of $0.4 \AA /$ cycle and $1.2 \AA /$ cycle, respectively, after a $5 \mathrm{~s}$ pulse of $\mathbf{5}$ and $9 \mathrm{~s}$ pulse of $\mathrm{NH}_{3}$ plasma (Figure $5.4 \mathrm{~b}$ and 5.4c). These results indicate a self-limiting surface chemistry for $\mathbf{5}$ and $\mathrm{NH}_{3}$ plasma at both the lower and higher temperature regions. The high-temperature interval growth rate is three and four times that of our previous reports using $\operatorname{In}(\text { famd })_{3}$ $(0.4 \AA / \text { cycle })^{197}$ and $\mathrm{InMe}_{3}(0.32 \AA / \text { cycle })^{184}$, respectively, with $\mathrm{NH}_{3}$ plasma. 

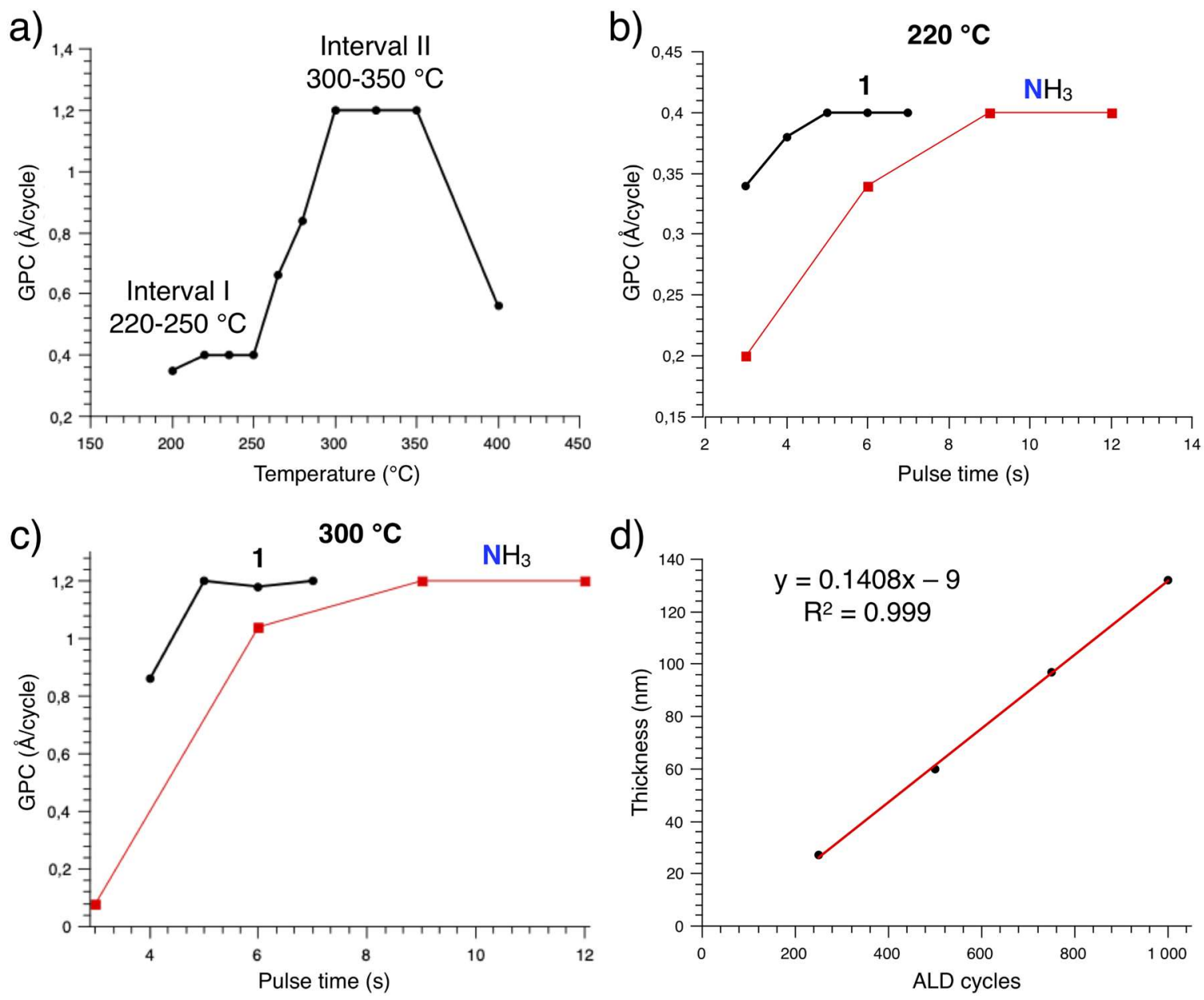

Figure 5.4: a) The growth dependence on process temperature using a $5 \mathrm{~s}$ pulse of $\mathbf{5}$ and 9 s pulse of $\mathrm{NH}_{3}$ on $\mathrm{Si}(100)$. b) The growth per cycle behavior of $\mathbf{5}$ and $\mathrm{NH}_{3}$ pulses deposited at $220{ }^{\circ} \mathrm{C}$ on $\mathrm{Si}(100)$. c) The growth per cycle behavior of $\mathbf{1}$ and $\mathrm{NH}_{3}$ pulses deposited at $300{ }^{\circ} \mathrm{C}$ on $\mathrm{Si}(100)$. d) Growth behavior of $\mathbf{5}$ at $300{ }^{\circ} \mathrm{C}$ dependent on the number of cycles using a 5 s pulse of $\mathbf{5}$ and a $9 \mathrm{~s} \mathrm{NH}_{3}$ plasma pulse on $\mathrm{Si}(100)$. The red line indicates the linear trend.

Furthermore, the high temperature interval indicates a higher thermal stability of the chemisorbed species in comparison to the previously used $\operatorname{In}(\text { famd })_{3}$, which has a constant GPC over a wide temperature range of $200-280{ }^{\circ} \mathrm{C} .{ }^{197} \mathrm{~A}$ linear growth trend was observed when all the parameters were kept constant upon increasing ALD cycles at 220 and $300{ }^{\circ} \mathrm{C}$ (Figure 5.13 and 5.4d). 


\section{Thermal Properties of In(III) Triazenide Precursor}

The presence of two temperature intervals with self-limiting deposition is high exceptional in ALD. Differential scanning calorimetry (DSC) analysis of $\mathbf{5}$ shows two exothermic events, a sharp peak between $150-180^{\circ} \mathrm{C}$ and a broad peak between 220 and $280{ }^{\circ} \mathrm{C}$ (Figure 5.10). The onset temperature of the first exothermic event coincides with the onset volatilization temperature observed in the TGA $\left(\sim 145^{\circ} \mathrm{C}\right)$, which could indicate decomposition and volatilization occurring simultaneously. Heating of $\mathbf{5}$ in solid state to $180{ }^{\circ} \mathrm{C}$ for $16 \mathrm{~h}$ showed only slight discoloration of the sample with no signs of decomposition by ${ }^{1} \mathrm{H}$ NMR analysis. These results suggest that the first exothermic event does not correspond to a decomposition pathway. The spike in growth rate between 250 and $300{ }^{\circ} \mathrm{C}$ lines up with the second broad exothermic event between 220 and $280{ }^{\circ} \mathrm{C}$, which contains three overlapping peaks at approximately 245,255 , and $270{ }^{\circ} \mathrm{C}$. Heating studies of $\mathbf{5}$ in solid and solution states (Figure 5.11) above $200{ }^{\circ} \mathrm{C}$ confirmed this to be a decomposition event, giving In metal and an unidentifiable brown solid. This is a strong indication that 1 decomposes in the gas-phase inside the deposition chamber to a more volatile and reactive species at $\sim 250{ }^{\circ} \mathrm{C}$. It is worth pointing out that even though this is usually an unwanted ALD precursor property, in this case the decomposition product still retains self-limiting growth behavior. 
The thermal decomposition of $\mathbf{5}$ was studied by DFT calculations at $300{ }^{\circ} \mathrm{C}$ showing two possible pathways (Figure 5.5a). The first common step of both pathways is a transfer of a hydrogen from the methyl group of the $i \operatorname{Pr}$ moiety to the endocyclic nitrogen, releasing a propene molecule to form $\mathbf{A}$. Compound $\mathbf{A}$ can tautomerize through a hydrogen transfer to the exocyclic nitrogen to give $\mathbf{B}$. The other possible path, which is more energetically favored, is migration of the hydrogen onto the exocyclic tertiary nitrogen while simultaneously releasing $\mathrm{N}_{2}$ to form $\mathbf{C}$. The free energies for the decomposition path are shown at $300{ }^{\circ} \mathrm{C}$ (Figure $5.5 \mathrm{~b})$. The energy for the final structure $\mathbf{C}\left(\Delta \mathrm{G}^{\circ}=-245 \mathrm{~kJ} \mathrm{~mol}^{-1}\right)$ is much lower than $\mathbf{5}$, due to the large increase of entropy when releasing two molecules. This indicates that a higher temperature and lower pressure structure $\mathbf{C}$ would be expected to dominate. Further calculations show that all three ligands decompose simultaneously via this pathway to give a tricoordinated $\operatorname{In}(\mathrm{III})$ species of $\mathbf{C}$ (Table 5.10 and 5.11). Decomposition of the triazenide ligand of $\mathbf{5}$ is uniquely different to that of amidinates and guanidinates, which have been shown to thermally decompose by either carbodiimide deinsertion or $\beta$-hydrogen abstraction. ${ }^{221}$ This highlights the reduced hydride reactivity of the $\beta$-hydrogen on the isopropyl moiety by the electron-withdrawing triazenide ligand and removes the need for a bulky tert-butyl exocyclic substituent to block this position. 
a)

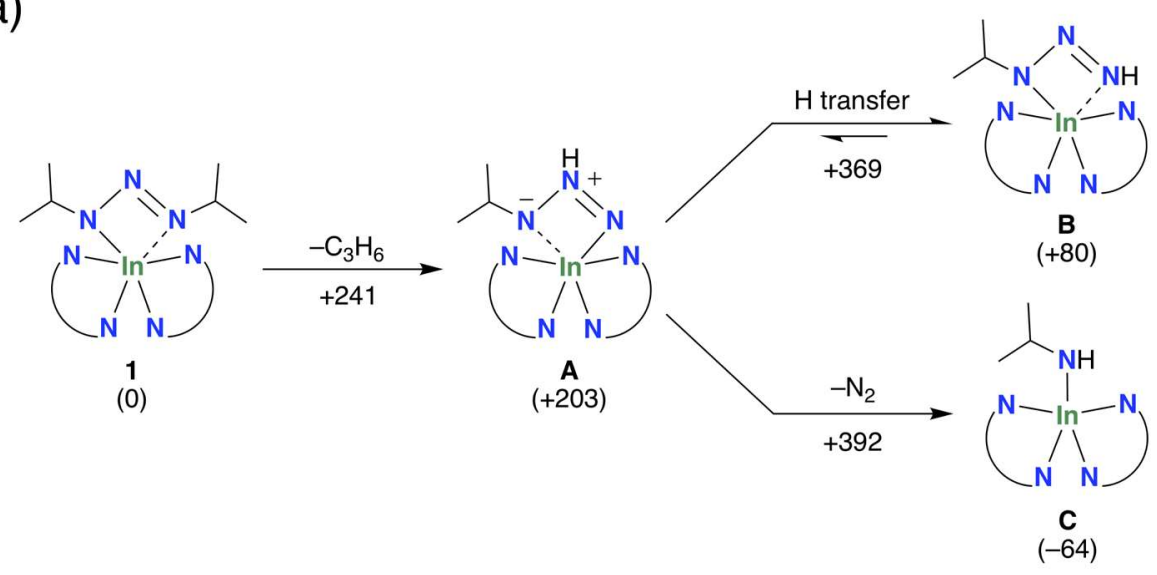

b)

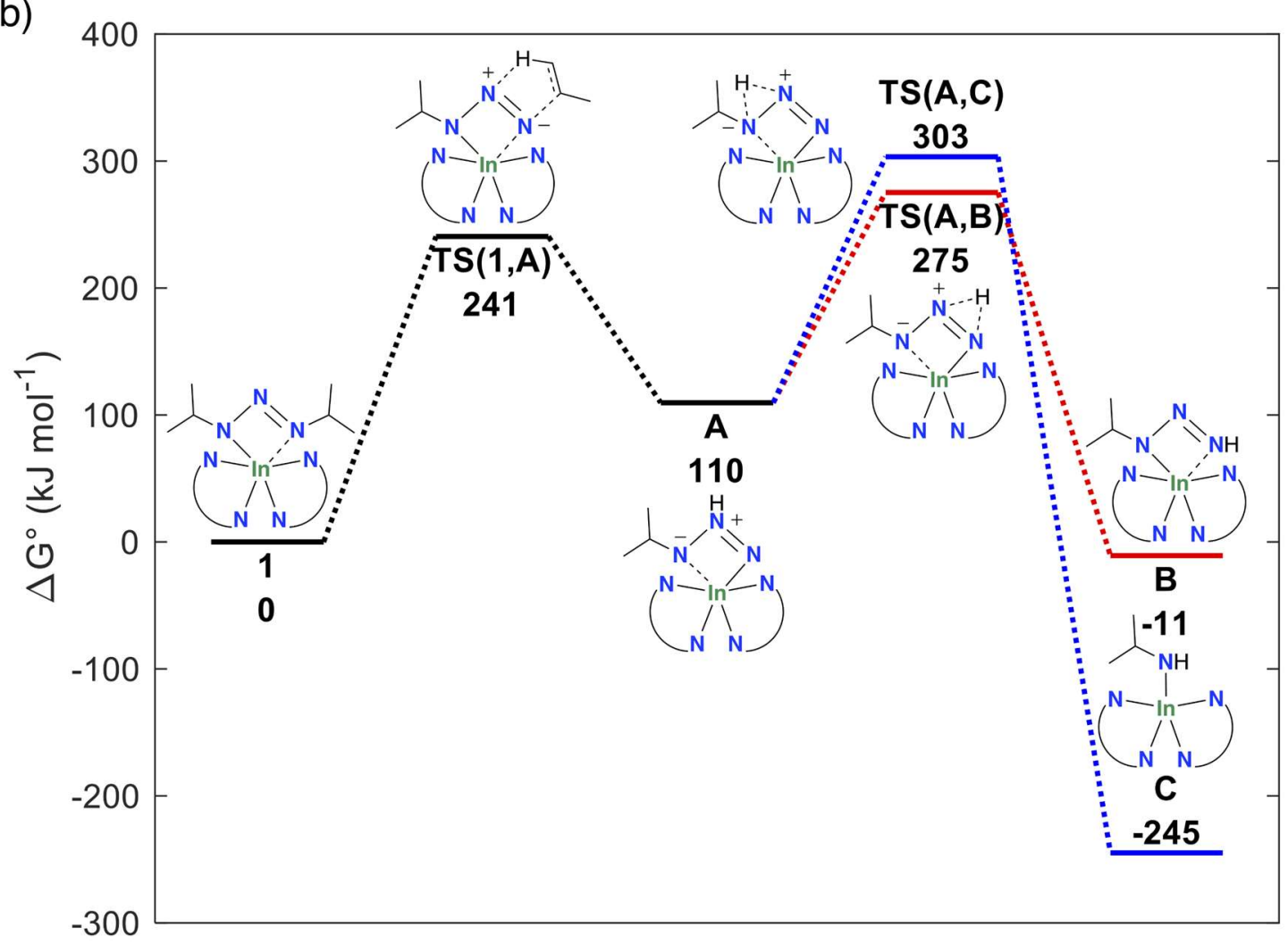

Figure 5.5: a) DFT calculated decomposition pathways for the thermal decomposition of $\mathbf{5}$ at $300{ }^{\circ} \mathrm{C}$. The change in enthalpy is shown in each step and is given in $\mathrm{kJ} \mathrm{mol}^{-1}$. b) The free energy profile for the decomposition of $\mathbf{5}$ at $300{ }^{\circ} \mathrm{C}$. The free energies are marked for each pathway and are given in $\mathrm{kJ} \mathrm{mol}^{-1}$. 


\section{InN Film Characterization}

The films deposited at both temperature intervals rendered polycrystalline hexagonal wurtzite $\mathrm{InN}$ on $\mathrm{Si}(100)$ (Figure 5.14a-c). Deposition on $4 \mathrm{H}-\mathrm{SiC}(0001)$, which has a smaller lattice mismatch with $\mathrm{InN}, 177$ at $325{ }^{\circ} \mathrm{C}$ with $5 \mathrm{~s}$ pulse of $\mathbf{5}$ and $9 \mathrm{~s} \mathrm{NH}_{3}$ plasma pulse rendered epitaxial wurtzite $\mathrm{InN}$. The $\vartheta$ - $2 \vartheta$ X-ray diffraction (XRD) measurement shows InN peaks corresponding to the (0002), (0004) and (0006) planes indicating growth along the c-axis (Figure 5.6a). Grazing incidence XRD (GIXRD) shows no peaks indicating no tilted grains (Figure 5.14d). XRD pole figures of the $\operatorname{InN}(10-11)$ and (10-12) peaks were constructed to study the inplane grain orientation and the crystal relationship between the film and the substrate (Figure 5.15 and 5.6b). Both pole figures show 12 poles, with 6 poles corresponding to $4 \mathrm{H}-\mathrm{SiC}($ hkil) and 6 poles to the $\mathrm{InN}$ film. This is expected for an epitaxially grown hexagonal film on a hexagonal substrate due to its six-folded symmetry. Note that in the (10-11) plane (Figure 5.15), the poles for the substrate and the InN film overlaps. A distinct difference between the substrate and the InN poles was observed for the (10-12) plane (Figure 5.6b), where the inner 6 poles correspond to the $\mathrm{InN}$ film and the outer 6 planes correspond to the $\mathrm{SiC}$ substrate. 
a)

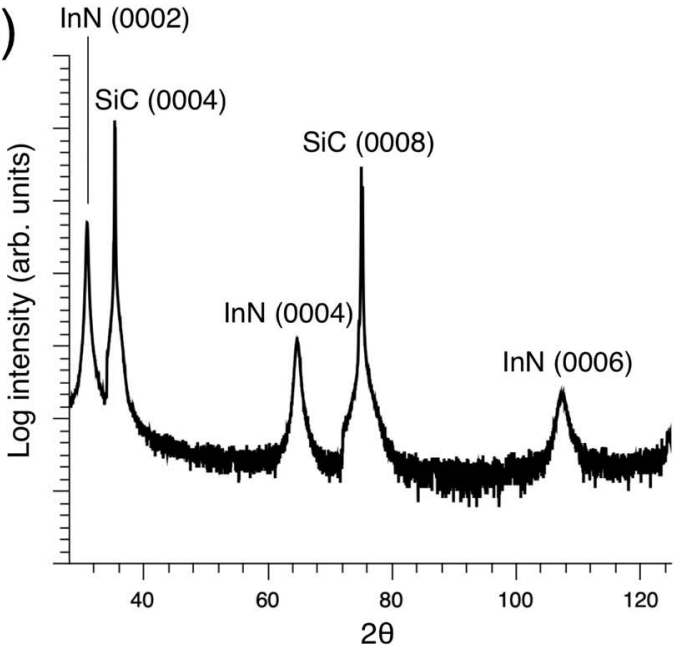

b)

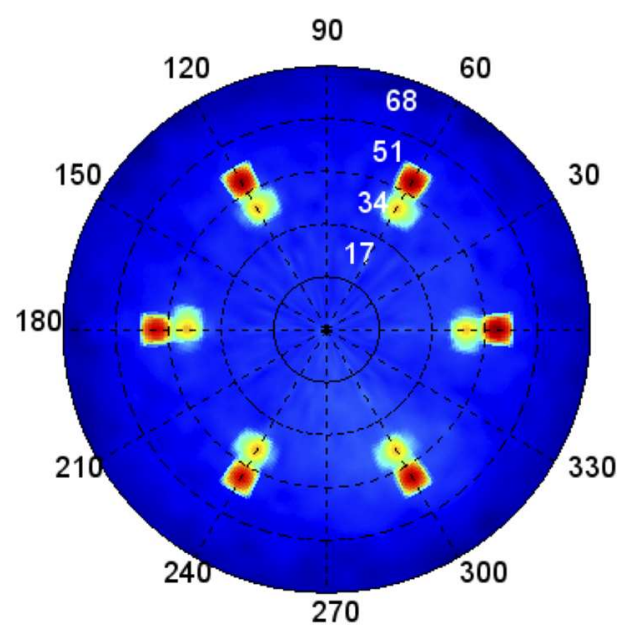

c)

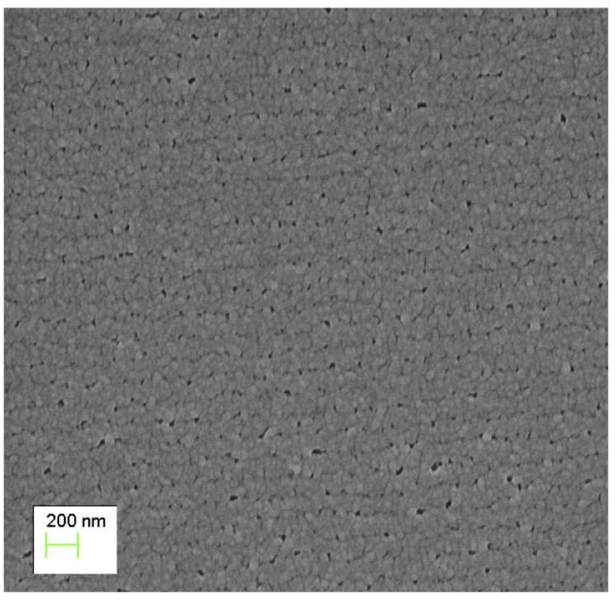

d)

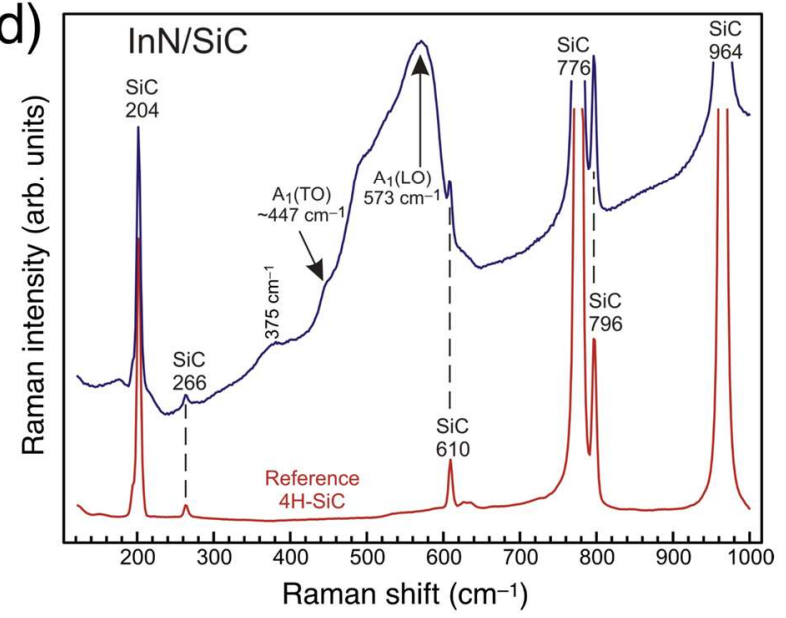

Figure 5.6: a) XRD of $\mathrm{InN}$ on $4 \mathrm{H}-\mathrm{SiC}(0001)$ using a $5 \mathrm{~s}$ pulse of $\mathbf{5}$ and $9 \mathrm{~s} \mathrm{NH}_{3}$ plasma pulse deposited at $325{ }^{\circ} \mathrm{C}$ showing epitaxial $\mathrm{InN}$ along the c-axis. b) Pole figure of the $\mathrm{InN}(10-12)$ plane showing 12 poles, the outer 6 red poles corresponding to $\mathrm{SiC}$ and the inner 6 green/yellow poles corresponding InN. c) Top view SEM of InN on $4 \mathrm{H}-\mathrm{SiC}$ deposited at $325{ }^{\circ} \mathrm{C}$. d) The Raman spectrum of InN on $4 \mathrm{H}-\mathrm{SiC}$ deposited at $325{ }^{\circ} \mathrm{C}$ together with the reference spectrum of the substrate provided for comparison. The lines belonging to the $\mathrm{SiC}$ substrate are labelled ' $\mathrm{SiC}$ ' with their corresponding Raman shifts in $\mathrm{cm}^{-1}$.

The $\vartheta-2 \vartheta$ XRD, GIXRD and the pole figure measurements shows that each InN hexagon grows epitaxially on a $\mathrm{SiC}$ hexagon, with in-plane relations $\mathrm{InN}$ [1011] || 4H-SiC [10-11] and $\mathrm{InN}$ [10-12] || 4H-SiC [10-12]. This confirms the epitaxial 
relation between the $\mathrm{InN}$ film and the $\mathrm{SiC}$ substrate along the c-axis; $\mathrm{InN}$ [0002] ॥ 4H-SiC [0001].

The top-view scanning electron microscope (SEM) image of the InN films on 4H-SiC shows homogenously ordered grains along horizontal lines with a line width of approximately $130 \mathrm{~nm}$ (Figure 5.6c). This indicates that the InN films grows on the terrace of the $4 \mathrm{H}-\mathrm{SiC}$ substrate, leaving partially uncoated stepedges. ${ }^{222}$ We speculate that the step-edges acts as a surface diffusion barrier for the atoms, forcing them to nucleate at the step-edge and the InN films starts to grow along the terrace. The species on the terrace have a higher surface diffusion compared to the species on the step allowing them to move towards the steps to nucleate. ${ }^{222,223}$ X-ray photoelectron spectroscopy (XPS) of the film deposited on $4 \mathrm{H}-\mathrm{SiC}$ at $325{ }^{\circ} \mathrm{C}$ with $5 \mathrm{~s}$ pulse of $\mathbf{5}$ and $9 \mathrm{~s} \mathrm{NH}_{3}$ plasma pulse showed $48.7 \pm 2.4$ at. $\%$ In, $48.6 \pm 2.4$ at. $\%$ N, $2.7 \pm 0.1$ at. $\% \mathrm{O}$ and no detectable C (Figure 5.18). The same amount of oxygen is bonded to In and $\mathrm{N}$, giving perfect $\mathrm{In} / \mathrm{N}$ ratio of 1.0. It should be noted that the $\mathrm{InN}$ film was capped with approximately $10 \mathrm{~nm}$ of AlN in order to avoid post deposition oxidation of the InN film. As preferential sputtering of $\mathrm{InN}$ can occur, this was minimized by applying the lowest sputtering energy and no tilting of the sample according to previously reported protocols. ${ }^{224}$ The high resolution XPS of In $3 \mathrm{~d}_{5 / 2}$ and N1s was fitted with two sub-peaks each. For In $3 \mathrm{~d}_{5 / 2}$, the major contribution at $444.6 \mathrm{eV}$ assigned to $\mathrm{In}-\mathrm{N}$ bond, with a 
minor contribution at $445.7 \mathrm{eV}$ assigned to the $\mathrm{In}-\mathrm{O}$ bond. For the $\mathrm{N}$ 1s, the major contribution at $396.5 \mathrm{eV}$ was assigned to $\mathrm{N}-\mathrm{In}$ bond, with a minor contribution at $397.6 \mathrm{eV}$ assigned to the $\mathrm{N}-\mathrm{O}$ bond. ${ }^{225-227}$ Raman spectroscopy of the InN films on $4 \mathrm{H}-\mathrm{SiC}$ deposited at $325{ }^{\circ} \mathrm{C}$ (Figure $5.6 \mathrm{~d}$ ) shows the $\mathrm{A}_{1}(\mathrm{LO})$ band of wurtzite $\mathrm{InN}$ at approximately $573 \mathrm{~cm}^{-1}$, which agrees well with previously reported Raman shift ${ }^{228}$ and indicates low stress in the film. A very small shoulder was observed at $375 \mathrm{~cm}^{-1}$, which is a gap mode previously shown to correspond to In vacancies in InN. ${ }^{228}$ The appearance of this gap mode is attributed to the small amount of oxygen in the film. The sheet resistance of $50 \mathrm{~nm}$ InN films is in average $920 \Omega / \mathrm{sq}$ which corresponds to a resistivity of $0.46 \Omega \mathrm{cm}$. This value is comparable to previously reported InN deposited by ALD at $240{ }^{\circ} \mathrm{C} .182$ We could not determine the carrier concentration and the carrier mobility with high accuracy due to the limitations of the instrument used. The fitted electron mobility values are $10-500$ $\mathrm{cm}^{2} /(\mathrm{V} \mathrm{s})$ depending on its correlated carrier concentration. We believe that this scattering is due to the confidence level of the fitting. Assuming a carrier concentration of $1 \times 10^{20} \mathrm{~cm}^{-3}$, the mobility is approximately $13 \mathrm{~cm}^{2} /(\mathrm{V} \mathrm{s})$. This value is in the same range as previously reported for InN films from ALD deposited at somewhat lower temperature. ${ }^{182}$ 


\subsubsection{Summary and Conclusions}

In conclusion, we have developed a new highly volatile $\mathrm{In}(\mathrm{III})$ precursor and shown for the first time the use of a homoleptic triazenide in a vapor deposition process. This compound was easily synthesized using cheap and easily obtainable reagents. Thermogravimetric analysis showed $\mathbf{5}$ underwent single step volatilization between 145 and $215{ }^{\circ} \mathrm{C}$ with negligible mass remaining and heating studies showed it started to slowly decompose at $\sim 200{ }^{\circ} \mathrm{C}$. Interestingly, $\mathbf{5}$ undergoes decomposition in the gas-phase at higher deposition temperatures to give a more reactive $\operatorname{In}(\mathrm{III})$ compound that still possess self-limiting behavior and without unwanted impurities in the film. DFT calculations showed $\mathbf{5}$ decomposes to a smaller tricoordinated In(III) compound, by liberating three molecules of propene and $\mathrm{N}_{2}$, and explains the increased growth rate observed in the higher temperature interval. This result has the potential to open a new path in ligand and precursor design for vapor deposition with deliberate gas-phase decomposition. ALD of $\mathrm{InN}$ using $\mathbf{5}$ and $\mathrm{NH}_{3}$ plasma afforded epitaxial $\mathrm{InN}$ on $4 \mathrm{H}-\mathrm{SiC}$ that was stoichiometric and with a very low level of impurities. Initial electrical characterization of the InN films shows a sheet resistivity of $920 \Omega^{2}$ and electron mobility on par with the reported literature. This new triazenide ligand has potential to unlock ALD of InN for semi-conductor applications and develop a new class of $\mathrm{M}-\mathrm{N}$ bonded precursors for future ALD processes. 


\subsubsection{Acknowledgements}

The authors acknowledge Laurent Souqui for his assistance with the pole figure measurements and Seán Barry for access to TGA and DSC instruments. This project was founded by the Swedish foundation for Strategic Research through the project "Time-resolved low temperature CVD for III-nitrides" (SSF-RMA 15-0018) and by the Knut and Alice Wallenberg foundation through the project "Bridging the THz gap" (No. KAW 2013.0049). L.O. acknowledges financial support from the Swedish Government Strategic Research Area in Materials Science on Functional Materials at Linköping University (Faculty Grant SFO Mat LiU no. 2009 00971). Supercomputing resources were provided by the Swedish National Infrastructure for Computing (SNIC) and the Swedish National Supercomputer Centre (NSC). 


\subsubsection{Supporting information}

\section{NMR Spectral Charts}

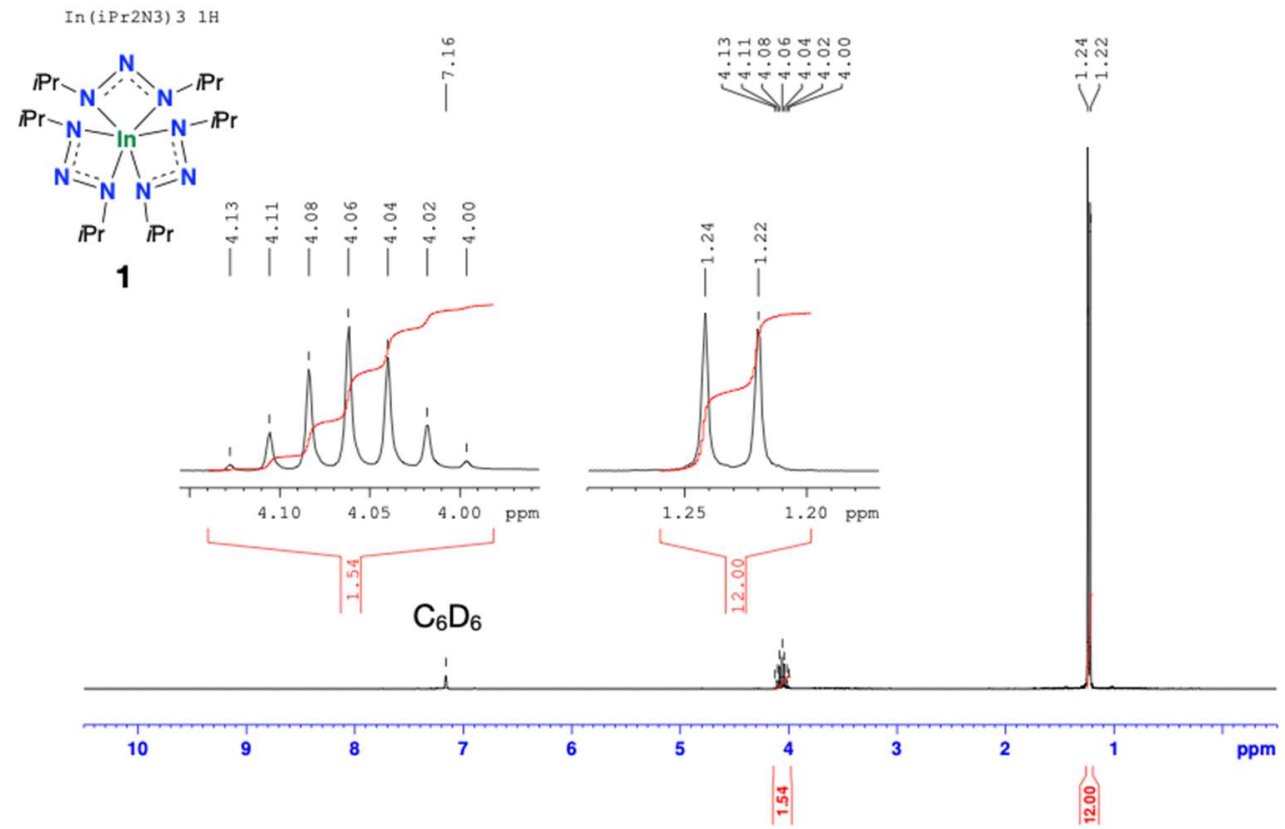

Figure 5.7: ${ }^{1} \mathrm{H}$ NMR $(300 \mathrm{MHz})$ spectrum of 5 in $\mathrm{C}_{6} \mathrm{D}_{6}$.

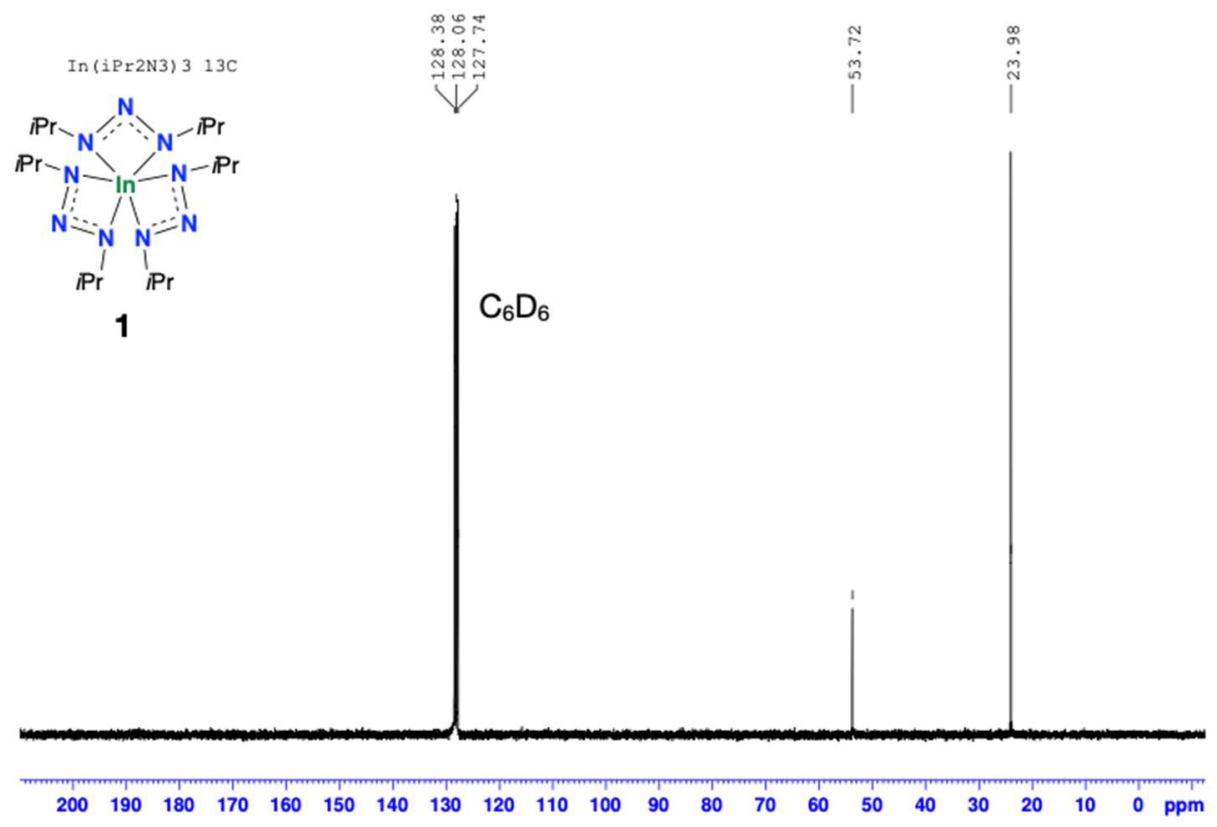

Figure 5.8: ${ }^{13} \mathrm{C}\left\{{ }^{1} \mathrm{H}\right\}$ NMR $(75 \mathrm{MHz})$ spectrum of 5 in $\mathrm{C}_{6} \mathrm{D}_{6}$. 


\section{Thermogravimetric Analysis}

(a)

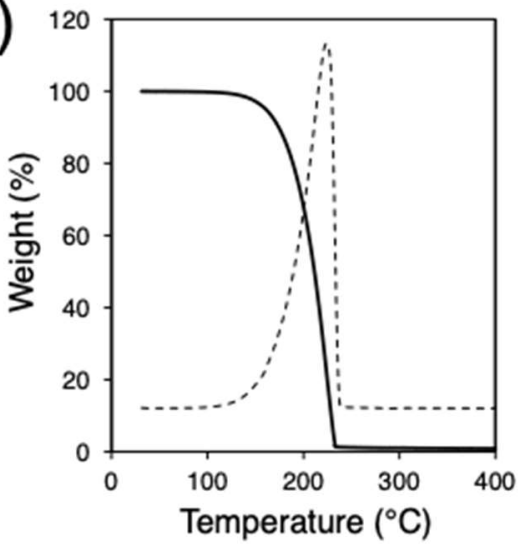

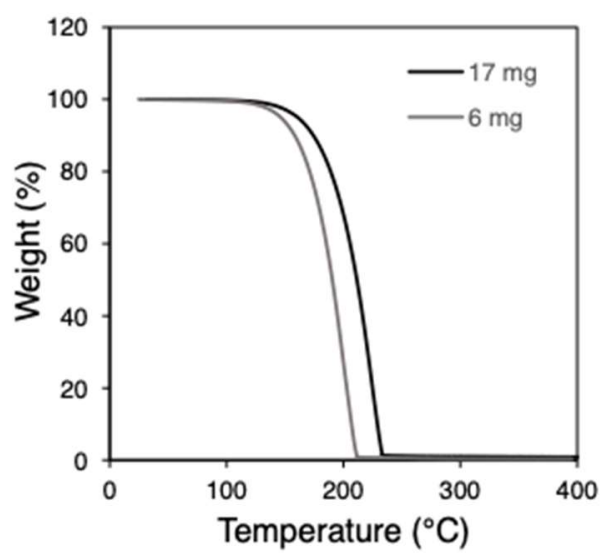

(b)

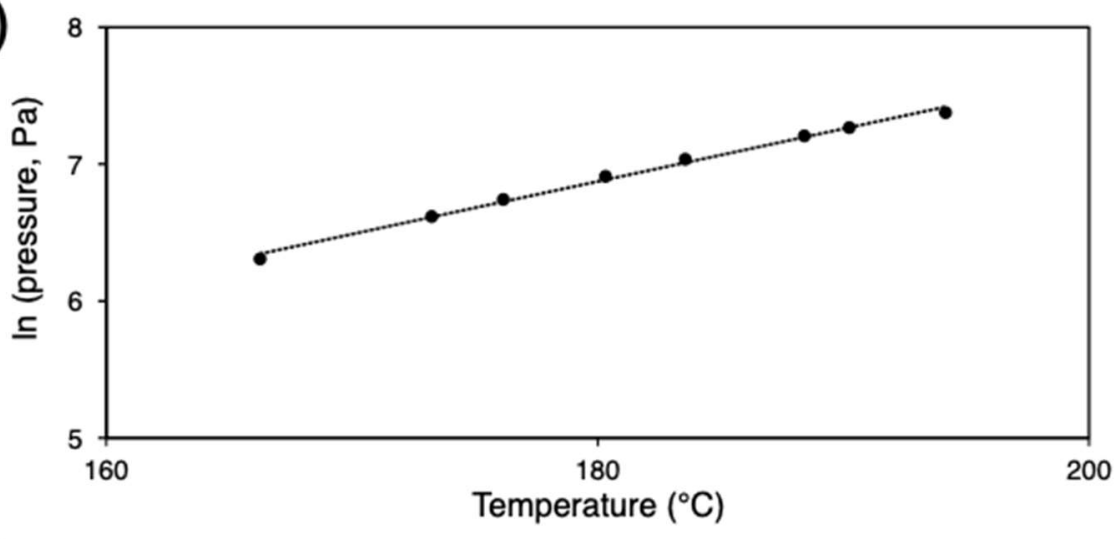

(c)

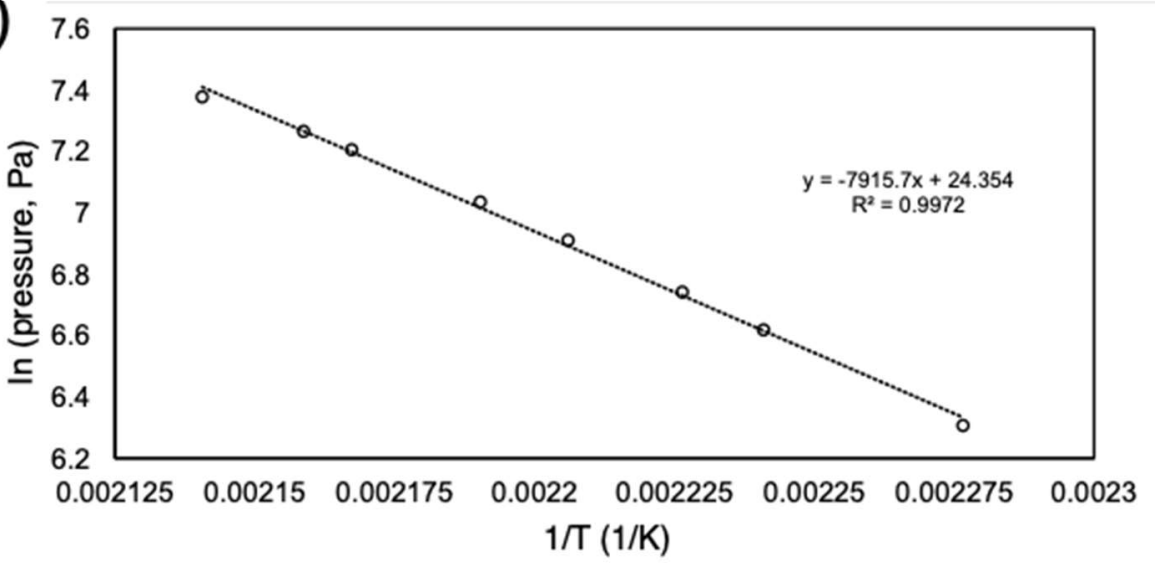

Figure 5.9: a) Thermogravimetric (left) and stress test analysis (right) of $\mathbf{5}$ and bc) its 1 Torr vapor pressure curves. 


\section{Differential Scanning Calorimetry Analysis}

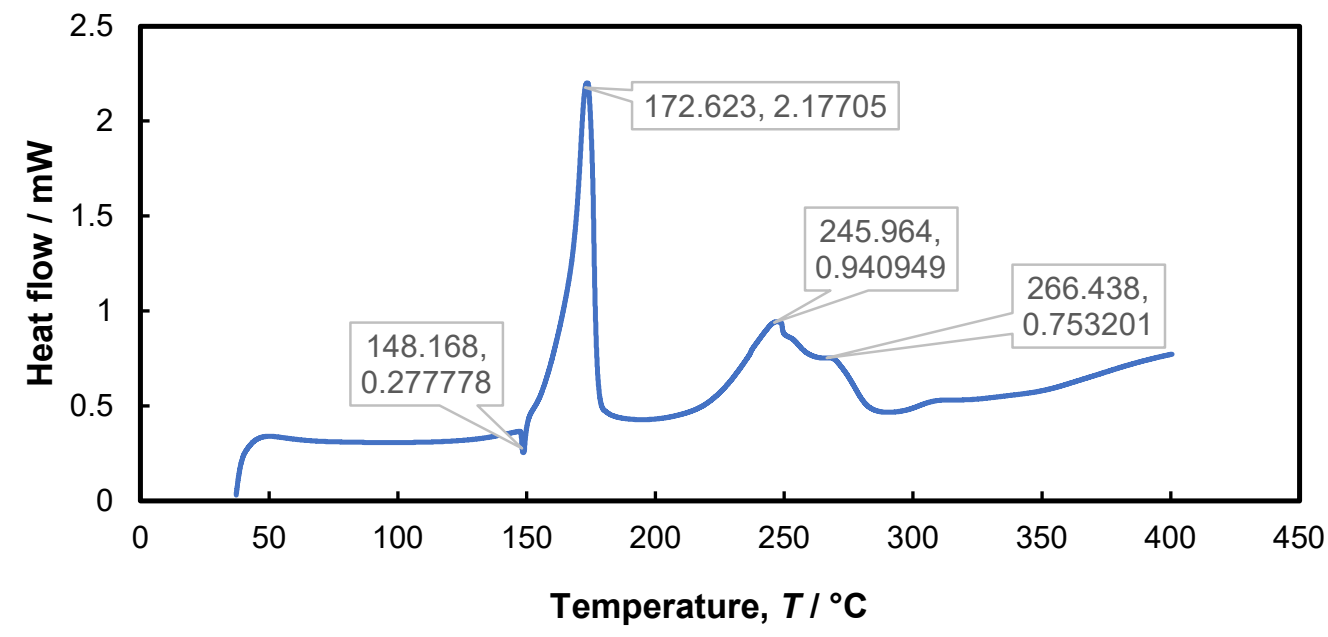

Figure 5.10: Differential scanning calorimetry of 5, which shows two distinct exothermic events between $150-180$ and $220-280^{\circ} \mathrm{C}$.

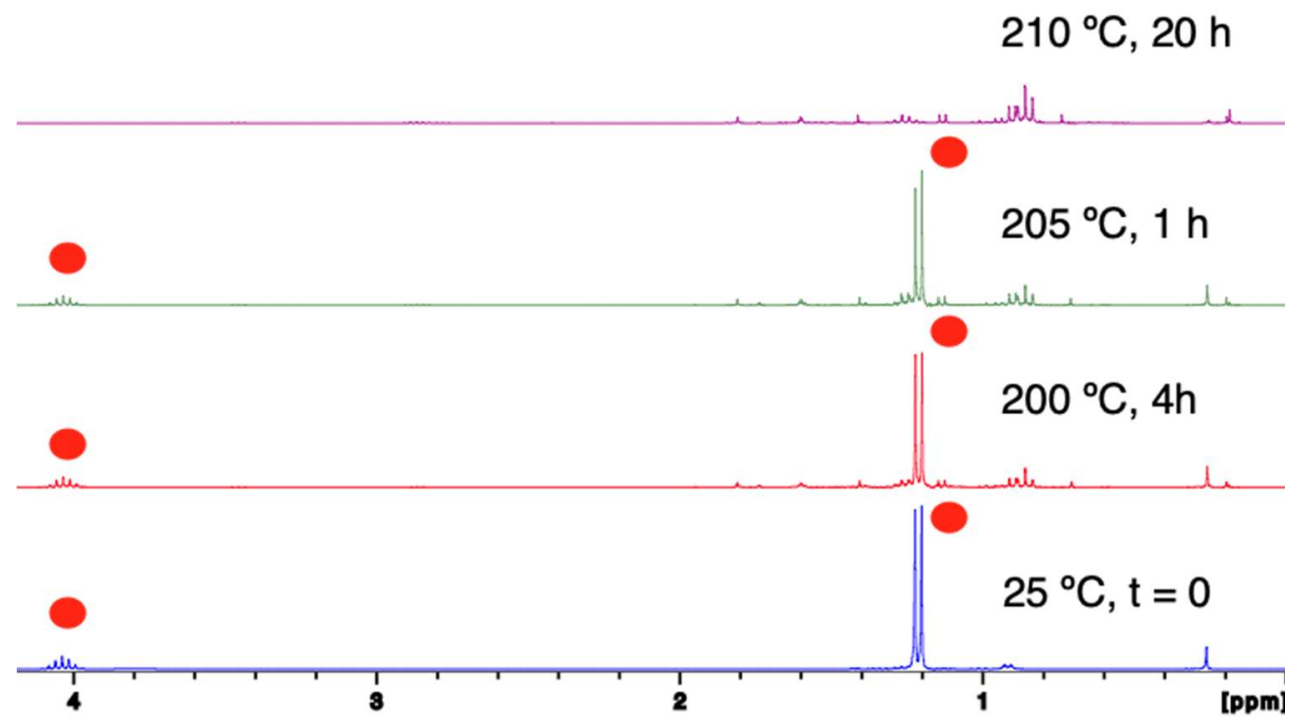

Figure 5.11: ${ }^{1} \mathrm{H}$ NMR spectra of 5, recorded during the decomposition study performed at various temperatures at $200{ }^{\circ} \mathrm{C}$ and above for different periods of time. Red dots show peaks of 5 . 


\section{X-ray Crystallographic Analysis}

Table 5.1: Crystal data and structure refinement for $\mathbf{5}$.

\begin{tabular}{|c|c|}
\hline Identification code & inpp2n3 \\
\hline Empirical formula & C18 H42 In N9 \\
\hline Formula weight & 499.43 \\
\hline Temperature & $296(2) \mathrm{K}$ \\
\hline Wavelength & $0.71073 \AA$ \\
\hline Crystal system & Orthorhombic \\
\hline Space group & Pnma \\
\hline Unit cell dimensions & $\begin{array}{l}\mathrm{a}=17.575(8) \AA \\
\mathrm{a}=90^{\circ} . \\
\mathrm{b}=16.067(7) \AA \\
\mathrm{b}=90^{\circ} . \\
\mathrm{c}=9.310(4) \AA \\
\mathrm{g}=90^{\circ} .\end{array}$ \\
\hline Volume & $2629(2) \AA^{3}$ \\
\hline $\mathrm{Z}$ & 4 \\
\hline Density (calculated) & $1.262 \mathrm{Mg} / \mathrm{m}^{3}$ \\
\hline Absorption coefficient & $0.919 \mathrm{~mm}^{-1}$ \\
\hline $\mathrm{F}(000)$ & 1048 \\
\hline Crystal size & $0.33 \times 0.24 \times 0.16 \mathrm{~mm}^{3}$ \\
\hline Theta range for data collection & 2.78 to $25.25^{\circ}$. \\
\hline Index ranges & $\begin{array}{l}-21<=\mathrm{h}<=21,- \\
19<=\mathrm{k}<=18,- \\
11<=\mathrm{l}<=11\end{array}$ \\
\hline Reflections collected & 14756 \\
\hline Independent reflections & $2424[\mathrm{R}(\mathrm{int})=0.0412]$ \\
\hline Completeness to theta $=25.25 \infty$ & $97.8 \%$ \\
\hline Absorption correction & None \\
\hline Max. and min. transmission & 0.8669 and 0.7513 \\
\hline Refinement method & $\begin{array}{l}\text { Full-matrix least-squares } \\
\text { on } \mathrm{F}^{2}\end{array}$ \\
\hline Data / restraints / parameters & $2424 / 290 / 184$ \\
\hline Goodness-of-fit on $\mathrm{F}^{2}$ & 0.911 \\
\hline Final $R$ indices $[I>2 \operatorname{sigma}(I)]$ & $\begin{array}{l}\mathrm{R} 1=0.0868, \mathrm{wR} 2= \\
0.2123\end{array}$ \\
\hline $\mathrm{R}$ indices (all data) & $\begin{array}{l}\mathrm{R} 1=0.0983, \mathrm{wR} 2= \\
0.2184\end{array}$ \\
\hline Largest diff. peak and hole & 0.658 and -0.770 e. $\AA^{-3}$ \\
\hline
\end{tabular}


Table 5.2: Atomic coordinates $\left(\mathrm{x} 10^{4}\right)$ and equivalent isotropic displacement parameters $\left(\AA^{2} \times 10^{3}\right)$ for $\mathbf{5}$. $\mathrm{U}(\mathrm{eq})$ is defined as one third of the trace of the orthogonalized Uij tensor.

\begin{tabular}{|c|c|c|c|c|}
\hline & $\mathrm{x}$ & $\mathrm{y}$ & $\mathrm{z}$ & $\mathrm{U}(\mathrm{eq})$ \\
\hline $\operatorname{In}(1)$ & $1250(1)$ & 2500 & $8876(1)$ & $75(1)$ \\
\hline $\mathrm{C}(2)$ & $2369(8)$ & $827(9)$ & 10327(17) & $140(3)$ \\
\hline $\mathrm{N}(3)$ & $1166(13)$ & $1650(20)$ & $11260(20)$ & $144(6)$ \\
\hline $\mathrm{N}(4)$ & $34(16)$ & 2500 & $6750(40)$ & $266(12)$ \\
\hline $\mathrm{C}(5)$ & $2554(17)$ & 2500 & $5970(30)$ & $183(8)$ \\
\hline $\mathrm{N}(6)$ & $2230(20)$ & $2200(20)$ & $7380(50)$ & $149(8)$ \\
\hline $\mathrm{N}(7)$ & $460(30)$ & $1860(30)$ & $7180(40)$ & $195(7)$ \\
\hline $\mathrm{N}(8)$ & $2050(30)$ & $1463(17)$ & $9420(50)$ & $124(3)$ \\
\hline $\mathrm{N}(9)$ & $770(20)$ & 2500 & $11020(30)$ & $141(6)$ \\
\hline $\mathrm{N}(10)$ & $1720(20)$ & $1610(30)$ & $10220(50)$ & $120(3)$ \\
\hline $\mathrm{C}(11)$ & $176(18)$ & 2500 & $12090(30)$ & $198(9)$ \\
\hline $\mathrm{N}(11)$ & $2482(13)$ & $1524(17)$ & $8010(30)$ & $153(7)$ \\
\hline $\mathrm{C}(13)$ & $-410(20)$ & $520(30)$ & $8450(40)$ & $212(9)$ \\
\hline $\mathrm{C}(21)$ & $-510(30)$ & $1730(30)$ & $11550(50)$ & $235(13)$ \\
\hline $\mathrm{C}(5 \mathrm{~A})$ & $2740(30)$ & $1680(30)$ & $5850(40)$ & $207(12)$ \\
\hline $\mathrm{N}(7 \mathrm{~A})$ & $320(20)$ & $1780(30)$ & $7910(40)$ & $185(6)$ \\
\hline $\mathrm{C}(14)$ & $1866(11)$ & $81(10)$ & $10700(20)$ & $185(4)$ \\
\hline $\mathrm{C}(21 \mathrm{~A})$ & $300(30)$ & $1750(30)$ & $12670(50)$ & $226(12)$ \\
\hline $\mathrm{C}(7)$ & $2030(30)$ & $1830(30)$ & $4840(40)$ & $224(14)$ \\
\hline $\mathrm{C}(13 \mathrm{~A})$ & 692(19) & $350(20)$ & $6870(40)$ & $202(8)$ \\
\hline $\mathrm{C}(24)$ & $3023(11)$ & $1315(13)$ & $10813(19)$ & $183(5)$ \\
\hline $\mathrm{C}(18)$ & $37(11)$ & $950(13)$ & $7240(20)$ & $193(6)$ \\
\hline
\end{tabular}


Table 5.3: Bond lengths $[\AA]$ and angles [] for $\mathbf{5}$.

\begin{tabular}{|c|c|c|c|}
\hline $\operatorname{In}(1)-N(7 A)$ & $2.19(3)$ & $\mathrm{C}(5)-\mathrm{C}(7) \# 1$ & $1.77(5)$ \\
\hline $\operatorname{In}(1)-\mathrm{N}(7 \mathrm{~A}) \# 1$ & $2.19(3)$ & $\mathrm{N}(6)-\mathrm{N}(6) \# 1$ & $0.96(8)$ \\
\hline $\operatorname{In}(1)-\mathrm{N}(9)$ & $2.17(3)$ & $\mathrm{N}(6)-\mathrm{N}(11)$ & $1.32(4)$ \\
\hline $\operatorname{In}(1)-\mathrm{N}(9) \# 1$ & $2.17(3)$ & $\mathrm{N}(6)-\mathrm{C}(5 \mathrm{~A})$ & $1.88(5)$ \\
\hline $\operatorname{In}(1)-\mathrm{N}(10)$ & $2.07(4)$ & $\mathrm{N}(7)-\mathrm{N}(7 \mathrm{~A})$ & $0.73(6)$ \\
\hline $\operatorname{In}(1)-\mathrm{N}(10) \# 1$ & $2.07(4)$ & $\mathrm{N}(7)-\mathrm{C}(18)$ & $1.64(4)$ \\
\hline $\operatorname{In}(1)-\mathrm{N}(8) \# 1$ & $2.24(4)$ & $\mathrm{N}(8)-\mathrm{N}(10)$ & $0.97(5)$ \\
\hline $\operatorname{In}(1)-\mathrm{N}(8)$ & $2.24(4)$ & $\mathrm{N}(8)-\mathrm{N}(11)$ & $1.52(5)$ \\
\hline $\operatorname{In}(1)-\mathrm{N}(6)$ & $2.26(3)$ & $\mathrm{N}(9)-\mathrm{N}(9) \# 1$ & $0.00003(10)$ \\
\hline $\operatorname{In}(1)-\mathrm{N}(6) \# 1$ & $2.26(3)$ & $\mathrm{N}(9)-\mathrm{C}(11)$ & $1.44(3)$ \\
\hline $\operatorname{In}(1)-\mathrm{N}(7)$ & $2.34(3)$ & $\mathrm{N}(9)-\mathrm{N}(3) \# 1$ & $1.55(3)$ \\
\hline $\operatorname{In}(1)-\mathrm{N}(7) \# 1$ & $2.34(3)$ & $\mathrm{C}(11)-\mathrm{C}(21 \mathrm{~A}) \# 1$ & $1.33(5)$ \\
\hline $\mathrm{C}(2)-\mathrm{C}(24)$ & $1.46(2)$ & $\mathrm{C}(11)-\mathrm{C}(21 \mathrm{~A})$ & $1.33(5)$ \\
\hline $\mathrm{C}(2)-\mathrm{N}(8)$ & $1.44(3)$ & $\mathrm{C}(11)-\mathrm{N}(9) \# 1$ & $1.44(3)$ \\
\hline $\mathrm{C}(2)-\mathrm{N}(10)$ & $1.70(4)$ & $\mathrm{C}(11)-\mathrm{C}(21) \# 1$ & $1.80(5)$ \\
\hline $\mathrm{C}(2)-\mathrm{C}(14)$ & $1.531(10)$ & $\mathrm{C}(11)-\mathrm{C}(21)$ & $1.80(5)$ \\
\hline $\mathrm{N}(3)-\mathrm{N}(10)$ & $1.37(5)$ & $\mathrm{C}(13)-\mathrm{C}(18)$ & $1.535(10)$ \\
\hline $\mathrm{N}(3)-\mathrm{N}(9)$ & $1.55(3)$ & $\mathrm{C}(21)-\mathrm{C}(21) \# 1$ & $2.47(11)$ \\
\hline $\mathrm{N}(3)-\mathrm{N}(9) \# 1$ & $1.55(3)$ & $\mathrm{C}(21)-\mathrm{C}(21 \mathrm{~A})$ & $1.77(6)$ \\
\hline $\mathrm{N}(4)-\mathrm{N}(7) \# 1$ & $1.34(4)$ & $\mathrm{C}(5 \mathrm{~A})-\mathrm{C}(7)$ & $1.58(6)$ \\
\hline $\mathrm{N}(4)-\mathrm{N}(7)$ & $1.34(4)$ & $\mathrm{N}(7 \mathrm{~A})-\mathrm{C}(18)$ & $1.56(4)$ \\
\hline $\mathrm{N}(4)-\mathrm{N}(7 \mathrm{~A})$ & $1.66(4)$ & $\mathrm{C}(7)-\mathrm{C}(7) \# 1$ & $2.16(10)$ \\
\hline $\mathrm{N}(4)-\mathrm{N}(7 \mathrm{~A}) \# 1$ & $1.66(4)$ & $\mathrm{C}(13 \mathrm{~A})-\mathrm{C}(18)$ & $1.537(10)$ \\
\hline $\mathrm{C}(5)-\mathrm{C}(5 \mathrm{~A})$ & $1.35(5)$ & $\mathrm{N}(7 \mathrm{~A})-\operatorname{In}(1)-\mathrm{N}(7 \mathrm{~A}) \# 1$ & $63(2)$ \\
\hline $\mathrm{C}(5)-\mathrm{C}(5 \mathrm{~A}) \# 1$ & $1.35(5)$ & $\mathrm{N}(7 \mathrm{~A})-\operatorname{In}(1)-\mathrm{N}(9)$ & $94.9(14)$ \\
\hline $\mathrm{C}(5)-\mathrm{N}(6) \# 1$ & $1.51(4)$ & $\mathrm{N}(7 \mathrm{~A}) \# 1-\operatorname{In}(1)-\mathrm{N}(9)$ & $94.9(14)$ \\
\hline $\mathrm{C}(5)-\mathrm{N}(6)$ & $1.51(4)$ & $\mathrm{N}(7 \mathrm{~A})-\operatorname{In}(1)-\mathrm{N}(9) \# 1$ & $94.9(14)$ \\
\hline $\mathrm{C}(5)-\mathrm{C}(7)$ & $1.77(5)$ & $\mathrm{N}(7 \mathrm{~A}) \# 1-\operatorname{In}(1)-\mathrm{N}(9) \# 1$ & $94.9(14)$ \\
\hline
\end{tabular}




\begin{tabular}{|c|c|c|c|}
\hline $\mathrm{N}(9)-\operatorname{In}(1)-\mathrm{N}(9) \# 1$ & $0.001(6)$ & $\mathrm{N}(7 \mathrm{~A})-\operatorname{In}(1)-\mathrm{N}(6) \# 1$ & $115.2(16)$ \\
\hline $\mathrm{N}(7 \mathrm{~A})-\operatorname{In}(1)-\mathrm{N}(10)$ & 100.3(16) & $\mathrm{N}(7 \mathrm{~A}) \# 1-\operatorname{In}(1)-\mathrm{N}(6) \# 1$ & $101.7(18)$ \\
\hline $\mathrm{N}(7 \mathrm{~A}) \# 1-\operatorname{In}(1)-\mathrm{N}(10)$ & $155.1(17)$ & $\mathrm{N}(9)-\operatorname{In}(1)-\mathrm{N}(6) \# 1$ & $149.7(16)$ \\
\hline $\mathrm{N}(9)-\operatorname{In}(1)-\mathrm{N}(10)$ & $66.4(15)$ & $\mathrm{N}(9) \# 1-\operatorname{In}(1)-\mathrm{N}(6) \# 1$ & $149.7(16)$ \\
\hline $\mathrm{N}(9) \# 1-\operatorname{In}(1)-\mathrm{N}(10)$ & $66.4(15)$ & $\mathrm{N}(10)-\operatorname{In}(1)-\mathrm{N}(6) \# 1$ & $102.5(18)$ \\
\hline $\mathrm{N}(7 \mathrm{~A})-\operatorname{In}(1)-\mathrm{N}(10) \# 1$ & $155.1(17)$ & $\mathrm{N}(10) \# 1-\operatorname{In}(1)-\mathrm{N}(6) \# 1$ & $85.6(18)$ \\
\hline $\mathrm{N}(7 \mathrm{~A}) \# 1-\operatorname{In}(1)-\mathrm{N}(10) \# 1$ & $100.3(16)$ & $\mathrm{N}(8) \# 1-\operatorname{In}(1)-\mathrm{N}(6) \# 1$ & $60.3(15)$ \\
\hline $\mathrm{N}(9)-\operatorname{In}(1)-\mathrm{N}(10) \# 1$ & $66.4(15)$ & $\mathrm{N}(8)-\operatorname{In}(1)-\mathrm{N}(6) \# 1$ & $79.6(15)$ \\
\hline $\mathrm{N}(9) \# 1-\operatorname{In}(1)-\mathrm{N}(10) \# 1$ & $66.4(15)$ & $\mathrm{N}(6)-\operatorname{In}(1)-\mathrm{N}(6) \# 1$ & $24(2)$ \\
\hline $\mathrm{N}(10)-\operatorname{In}(1)-\mathrm{N}(10) \# 1$ & $88(2)$ & $\mathrm{N}(7 \mathrm{~A})-\operatorname{In}(1)-\mathrm{N}(7)$ & $18.2(16)$ \\
\hline $\mathrm{N}(7 \mathrm{~A})-\operatorname{In}(1)-\mathrm{N}(8) \# 1$ & $162.4(15)$ & $\mathrm{N}(7 \mathrm{~A}) \# 1-\operatorname{In}(1)-\mathrm{N}(7)$ & $61.0(11)$ \\
\hline $\mathrm{N}(7 \mathrm{~A}) \# 1-\operatorname{In}(1)-\mathrm{N}(8) \# 1$ & $99.8(16)$ & $\mathrm{N}(9)-\operatorname{In}(1)-\mathrm{N}(7)$ & $112.9(15)$ \\
\hline $\mathrm{N}(9)-\operatorname{In}(1)-\mathrm{N}(8) \# 1$ & $92.1(13)$ & $\mathrm{N}(9) \# 1-\operatorname{In}(1)-\mathrm{N}(7)$ & $112.9(15)$ \\
\hline $\mathrm{N}(9) \# 1-\operatorname{In}(1)-\mathrm{N}(8) \# 1$ & $92.1(13)$ & $\mathrm{N}(10)-\operatorname{In}(1)-\mathrm{N}(7)$ & $109.6(16)$ \\
\hline $\mathrm{N}(10)-\operatorname{In}(1)-\mathrm{N}(8) \# 1$ & $97.3(14)$ & $\mathrm{N}(10) \# 1-\operatorname{In}(1)-\mathrm{N}(7)$ & $161.3(16)$ \\
\hline $\mathrm{N}(10) \# 1-\operatorname{In}(1)-\mathrm{N}(8) \# 1$ & $25.7(13)$ & $\mathrm{N}(8) \# 1-\operatorname{In}(1)-\mathrm{N}(7)$ & $148.7(15)$ \\
\hline $\mathrm{N}(7 \mathrm{~A})-\operatorname{In}(1)-\mathrm{N}(8)$ & $99.9(16)$ & $\mathrm{N}(8)-\operatorname{In}(1)-\mathrm{N}(7)$ & $101.4(16)$ \\
\hline $\mathrm{N}(7 \mathrm{~A}) \# 1-\operatorname{In}(1)-\mathrm{N}(8)$ & $162.4(15)$ & $\mathrm{N}(6)-\operatorname{In}(1)-\mathrm{N}(7)$ & $86.6(18)$ \\
\hline $\mathrm{N}(9)-\operatorname{In}(1)-\mathrm{N}(8)$ & $92.1(13)$ & $\mathrm{N}(6) \# 1-\operatorname{In}(1)-\mathrm{N}(7)$ & $97.4(17)$ \\
\hline $\mathrm{N}(9) \# 1-\operatorname{In}(1)-\mathrm{N}(8)$ & $92.1(13)$ & $\mathrm{N}(7 \mathrm{~A})-\operatorname{In}(1)-\mathrm{N}(7) \# 1$ & $61.0(11)$ \\
\hline $\mathrm{N}(10)-\operatorname{In}(1)-\mathrm{N}(8)$ & $25.7(13)$ & $\mathrm{N}(7 \mathrm{~A}) \# 1-\operatorname{In}(1)-\mathrm{N}(7) \# 1$ & 18.2(16) \\
\hline $\mathrm{N}(10) \# 1-\operatorname{In}(1)-\mathrm{N}(8)$ & $97.3(14)$ & $\mathrm{N}(9)-\operatorname{In}(1)-\mathrm{N}(7) \# 1$ & $112.9(15)$ \\
\hline $\mathrm{N}(8) \# 1-\operatorname{In}(1)-\mathrm{N}(8)$ & $96.0(19)$ & $\mathrm{N}(9) \# 1-\operatorname{In}(1)-\mathrm{N}(7) \# 1$ & $112.9(15)$ \\
\hline $\mathrm{N}(7 \mathrm{~A})-\mathrm{In}(1)-\mathrm{N}(6)$ & 101.7(18) & $\mathrm{N}(10)-\operatorname{In}(1)-\mathrm{N}(7) \# 1$ & $161.3(16)$ \\
\hline $\mathrm{N}(7 \mathrm{~A}) \# 1-\operatorname{In}(1)-\mathrm{N}(6)$ & $115.2(16)$ & $\mathrm{N}(10) \# 1-\operatorname{In}(1)-\mathrm{N}(7) \# 1$ & 109.6(16) \\
\hline $\mathrm{N}(9)-\operatorname{In}(1)-\mathrm{N}(6)$ & $149.7(16)$ & $\mathrm{N}(8) \# 1-\operatorname{In}(1)-\mathrm{N}(7) \# 1$ & 101.4(16) \\
\hline $\mathrm{N}(9) \# 1-\operatorname{In}(1)-\mathrm{N}(6)$ & $149.7(16)$ & $\mathrm{N}(8)-\operatorname{In}(1)-\mathrm{N}(7) \# 1$ & $148.7(15)$ \\
\hline $\mathrm{N}(10)-\operatorname{In}(1)-\mathrm{N}(6)$ & $85.6(17)$ & $\mathrm{N}(6)-\operatorname{In}(1)-\mathrm{N}(7) \# 1$ & $97.4(17)$ \\
\hline $\mathrm{N}(10) \# 1-\operatorname{In}(1)-\mathrm{N}(6)$ & $102.5(18)$ & $\mathrm{N}(6) \# 1-\operatorname{In}(1)-\mathrm{N}(7) \# 1$ & $86.6(18)$ \\
\hline $\mathrm{N}(8) \# 1-\operatorname{In}(1)-\mathrm{N}(6)$ & $79.6(15)$ & $\mathrm{N}(7)-\operatorname{In}(1)-\mathrm{N}(7) \# 1$ & $52(2)$ \\
\hline $\mathrm{N}(8)-\operatorname{In}(1)-\mathrm{N}(6)$ & $60.3(15)$ & $\mathrm{C}(24)-\mathrm{C}(2)-\mathrm{N}(8)$ & $96.0(18)$ \\
\hline
\end{tabular}




\begin{tabular}{|c|c|c|c|}
\hline $\mathrm{C}(24)-\mathrm{C}(2)-\mathrm{N}(10)$ & $98.8(18)$ & $\mathrm{C}(7)-\mathrm{C}(5)-\mathrm{C}(7) \# 1$ & $75(4)$ \\
\hline $\mathrm{N}(8)-\mathrm{C}(2)-\mathrm{N}(10)$ & $34.9(19)$ & $\mathrm{N}(6) \# 1-\mathrm{N}(6)-\mathrm{N}(11)$ & $146(3)$ \\
\hline $\mathrm{C}(24)-\mathrm{C}(2)-\mathrm{C}(14)$ & $143.4(16)$ & $\mathrm{N}(6) \# 1-\mathrm{N}(6)-\mathrm{C}(5)$ & $71.5(14)$ \\
\hline $\mathrm{N}(8)-\mathrm{C}(2)-\mathrm{C}(14)$ & $117.8(18)$ & $\mathrm{N}(11)-\mathrm{N}(6)-\mathrm{C}(5)$ & $121(3)$ \\
\hline $\mathrm{N}(10)-\mathrm{C}(2)-\mathrm{C}(14)$ & $101.7(18)$ & $\mathrm{N}(6) \# 1-\mathrm{N}(6)-\mathrm{C}(5 \mathrm{~A})$ & $116(2)$ \\
\hline $\mathrm{N}(10)-\mathrm{N}(3)-\mathrm{N}(9)$ & $106(2)$ & $\mathrm{N}(11)-\mathrm{N}(6)-\mathrm{C}(5 \mathrm{~A})$ & $79(3)$ \\
\hline $\mathrm{N}(10)-\mathrm{N}(3)-\mathrm{N}(9) \# 1$ & $106(2)$ & $\mathrm{C}(5)-\mathrm{N}(6)-\mathrm{C}(5 \mathrm{~A})$ & $45.5(19)$ \\
\hline $\mathrm{N}(9)-\mathrm{N}(3)-\mathrm{N}(9) \# 1$ & $0.001(8)$ & $\mathrm{N}(6) \# 1-\mathrm{N}(6)-\operatorname{In}(1)$ & $77.8(10)$ \\
\hline $\mathrm{N}(10)-\mathrm{N}(3)-\operatorname{In}(1)$ & $52.3(19)$ & $\mathrm{N}(11)-\mathrm{N}(6)-\operatorname{In}(1)$ & $99(2)$ \\
\hline $\mathrm{N}(9)-\mathrm{N}(3)-\operatorname{In}(1)$ & $56.2(12)$ & $\mathrm{C}(5)-\mathrm{N}(6)-\operatorname{In}(1)$ & $139(3)$ \\
\hline $\mathrm{N}(9) \# 1-\mathrm{N}(3)-\operatorname{In}(1)$ & $56.2(12)$ & $\mathrm{C}(5 \mathrm{~A})-\mathrm{N}(6)-\operatorname{In}(1)$ & $158(3)$ \\
\hline $\mathrm{N}(7) \# 1-\mathrm{N}(4)-\mathrm{N}(7)$ & $101(3)$ & $\mathrm{N}(7 \mathrm{~A})-\mathrm{N}(7)-\mathrm{N}(4)$ & $102(8)$ \\
\hline $\mathrm{N}(7) \# 1-\mathrm{N}(4)-\mathrm{N}(7 \mathrm{~A})$ & $99.8(18)$ & $\mathrm{N}(7 \mathrm{~A})-\mathrm{N}(7)-\mathrm{C}(18)$ & $71(5)$ \\
\hline $\mathrm{N}(7)-\mathrm{N}(4)-\mathrm{N}(7 \mathrm{~A})$ & $26(3)$ & $\mathrm{N}(4)-\mathrm{N}(7)-\mathrm{C}(18)$ & $116(3)$ \\
\hline $\mathrm{N}(7) \# 1-\mathrm{N}(4)-\mathrm{N}(7 \mathrm{~A}) \# 1$ & $26(3)$ & $\mathrm{N}(7 \mathrm{~A})-\mathrm{N}(7)-\operatorname{In}(1)$ & $69(4)$ \\
\hline $\mathrm{N}(7)-\mathrm{N}(4)-\mathrm{N}(7 \mathrm{~A}) \# 1$ & $99.8(18)$ & $\mathrm{N}(4)-\mathrm{N}(7)-\operatorname{In}(1)$ & $101(2)$ \\
\hline $\mathrm{N}(7 \mathrm{~A})-\mathrm{N}(4)-\mathrm{N}(7 \mathrm{~A}) \# 1$ & $88(2)$ & $\mathrm{C}(18)-\mathrm{N}(7)-\operatorname{In}(1)$ & $130(2)$ \\
\hline $\mathrm{C}(5 \mathrm{~A})-\mathrm{C}(5)-\mathrm{C}(5 \mathrm{~A}) \# 1$ & $151(5)$ & $\mathrm{N}(10)-\mathrm{N}(8)-\mathrm{N}(11)$ & $161(4)$ \\
\hline $\mathrm{C}(5 \mathrm{~A})-\mathrm{C}(5)-\mathrm{N}(6) \# 1$ & $118(3)$ & $\mathrm{N}(10)-\mathrm{N}(8)-\mathrm{C}(2)$ & $88(3)$ \\
\hline $\mathrm{C}(5 \mathrm{~A}) \# 1-\mathrm{C}(5)-\mathrm{N}(6) \# 1$ & $82(2)$ & $\mathrm{N}(11)-\mathrm{N}(8)-\mathrm{C}(2)$ & $111(3)$ \\
\hline $\mathrm{C}(5 \mathrm{~A})-\mathrm{C}(5)-\mathrm{N}(6)$ & $82(2)$ & $\mathrm{N}(10)-\mathrm{N}(8)-\operatorname{In}(1)$ & $67(3)$ \\
\hline $\mathrm{C}(5 \mathrm{~A}) \# 1-\mathrm{C}(5)-\mathrm{N}(6)$ & $118(3)$ & $\mathrm{N}(11)-\mathrm{N}(8)-\operatorname{In}(1)$ & $93.9(15)$ \\
\hline $\mathrm{N}(6) \# 1-\mathrm{C}(5)-\mathrm{N}(6)$ & $37(3)$ & $\mathrm{C}(2)-\mathrm{N}(8)-\operatorname{In}(1)$ & $155(3)$ \\
\hline $\mathrm{C}(5 \mathrm{~A})-\mathrm{C}(5)-\mathrm{C}(7)$ & $59(3)$ & $\mathrm{N}(9) \# 1-\mathrm{N}(9)-\mathrm{C}(11)$ & $37(10)$ \\
\hline $\mathrm{C}(5 \mathrm{~A}) \# 1-\mathrm{C}(5)-\mathrm{C}(7)$ & $132(4)$ & $\mathrm{N}(9) \# 1-\mathrm{N}(9)-\mathrm{N}(3)$ & $99(10)$ \\
\hline $\mathrm{N}(6) \# 1-\mathrm{C}(5)-\mathrm{C}(7)$ & $121(3)$ & $\mathrm{C}(11)-\mathrm{N}(9)-\mathrm{N}(3)$ & $103.2(15)$ \\
\hline $\mathrm{N}(6)-\mathrm{C}(5)-\mathrm{C}(7)$ & $97(3)$ & $\mathrm{N}(9) \# 1-\mathrm{N}(9)-\mathrm{N}(3) \# 1$ & $131(10)$ \\
\hline $\mathrm{C}(5 \mathrm{~A})-\mathrm{C}(5)-\mathrm{C}(7) \# 1$ & $132(4)$ & $\mathrm{C}(11)-\mathrm{N}(9)-\mathrm{N}(3) \# 1$ & $103.2(15)$ \\
\hline $\mathrm{C}(5 \mathrm{~A}) \# 1-\mathrm{C}(5)-\mathrm{C}(7) \# 1$ & $59(3)$ & $\mathrm{N}(3)-\mathrm{N}(9)-\mathrm{N}(3) \# 1$ & $123(3)$ \\
\hline $\mathrm{N}(6) \# 1-\mathrm{C}(5)-\mathrm{C}(7) \# 1$ & $97(3)$ & $\mathrm{N}(9) \# 1-\mathrm{N}(9)-\operatorname{In}(1)$ & $122(10)$ \\
\hline $\mathrm{N}(6)-\mathrm{C}(5)-\mathrm{C}(7) \# 1$ & $121(3)$ & $\mathrm{C}(11)-\mathrm{N}(9)-\operatorname{In}(1)$ & $157(3)$ \\
\hline
\end{tabular}




\begin{tabular}{|c|c|c|c|}
\hline $\mathrm{N}(3)-\mathrm{N}(9)-\operatorname{In}(1)$ & $87.4(13)$ & $\mathrm{N}(7)-\mathrm{N}(7 \mathrm{~A})-\mathrm{N}(4)$ & $52(6)$ \\
\hline $\mathrm{N}(3) \# 1-\mathrm{N}(9)-\operatorname{In}(1)$ & $87.4(13)$ & $\mathrm{C}(18)-\mathrm{N}(7 \mathrm{~A})-\mathrm{N}(4)$ & $103.7(18)$ \\
\hline $\mathrm{N}(8)-\mathrm{N}(10)-\mathrm{N}(3)$ & $167(5)$ & $\mathrm{N}(7)-\mathrm{N}(7 \mathrm{~A})-\operatorname{In}(1)$ & $93(5)$ \\
\hline $\mathrm{N}(8)-\mathrm{N}(10)-\mathrm{C}(2)$ & $58(3)$ & $\mathrm{C}(18)-\mathrm{N}(7 \mathrm{~A})-\operatorname{In}(1)$ & $149(3)$ \\
\hline $\mathrm{N}(3)-\mathrm{N}(10)-\mathrm{C}(2)$ & $118(3)$ & $\mathrm{N}(4)-\mathrm{N}(7 \mathrm{~A})-\operatorname{In}(1)$ & $97.5(19)$ \\
\hline $\mathrm{N}(8)-\mathrm{N}(10)-\operatorname{In}(1)$ & $87(3)$ & $\mathrm{C}(11)-\mathrm{C}(21 \mathrm{~A})-\mathrm{C}(21)$ & $69(3)$ \\
\hline $\mathrm{N}(3)-\mathrm{N}(10)-\operatorname{In}(1)$ & $96(2)$ & $\mathrm{C}(5 \mathrm{~A})-\mathrm{C}(7)-\mathrm{C}(5)$ & $47(2)$ \\
\hline $\mathrm{C}(2)-\mathrm{N}(10)-\operatorname{In}(1)$ & $144(3)$ & $\mathrm{C}(5 \mathrm{~A})-\mathrm{C}(7)-\mathrm{C}(7) \# 1$ & $98(3)$ \\
\hline $\mathrm{C}(21 \mathrm{~A}) \# 1-\mathrm{C}(11)-\mathrm{C}(21 \mathrm{~A})$ & $128(6)$ & $\mathrm{C}(5)-\mathrm{C}(7)-\mathrm{C}(7) \# 1$ & $52.4(19)$ \\
\hline $\mathrm{C}(21 \mathrm{~A}) \# 1-\mathrm{C}(11)-\mathrm{N}(9) \# 1$ & $99(2)$ & $\mathrm{C}(13)-\mathrm{C}(18)-\mathrm{C}(13 \mathrm{~A})$ & $105(3)$ \\
\hline $\mathrm{C}(21 \mathrm{~A})-\mathrm{C}(11)-\mathrm{N}(9) \# 1$ & $99(2)$ & $\mathrm{C}(13)-\mathrm{C}(18)-\mathrm{N}(7)$ & $132(3)$ \\
\hline $\mathrm{C}(21 \mathrm{~A}) \# 1-\mathrm{C}(11)-\mathrm{N}(9)$ & $99(2)$ & $\mathrm{C}(13 \mathrm{~A})-\mathrm{C}(18)-\mathrm{N}(7)$ & $102(3)$ \\
\hline $\mathrm{C}(21 \mathrm{~A})-\mathrm{C}(11)-\mathrm{N}(9)$ & $99(2)$ & $\mathrm{C}(13)-\mathrm{C}(18)-\mathrm{N}(7 \mathrm{~A})$ & $105(2)$ \\
\hline $\mathrm{N}(9) \# 1-\mathrm{C}(11)-\mathrm{N}(9)$ & $0.001(6)$ & $\mathrm{C}(13 \mathrm{~A})-\mathrm{C}(18)-\mathrm{N}(7 \mathrm{~A})$ & $113(2)$ \\
\hline $\mathrm{C}(21 \mathrm{~A}) \# 1-\mathrm{C}(11)-\mathrm{C}(21) \# 1$ & $67(3)$ & $\mathrm{N}(7)-\mathrm{C}(18)-\mathrm{N}(7 \mathrm{~A})$ & $26(2)$ \\
\hline $\mathrm{C}(21 \mathrm{~A})-\mathrm{C}(11)-\mathrm{C}(21) \# 1$ & $147(4)$ & & \\
\hline $\mathrm{N}(9) \# 1-\mathrm{C}(11)-\mathrm{C}(21) \# 1$ & $107(2)$ & & \\
\hline $\mathrm{N}(9)-\mathrm{C}(11)-\mathrm{C}(21) \# 1$ & $107(2)$ & & \\
\hline $\mathrm{C}(21 \mathrm{~A}) \# 1-\mathrm{C}(11)-\mathrm{C}(21)$ & $147(4)$ & & \\
\hline $\mathrm{C}(21 \mathrm{~A})-\mathrm{C}(11)-\mathrm{C}(21)$ & $67(3)$ & & \\
\hline $\mathrm{N}(9) \# 1-\mathrm{C}(11)-\mathrm{C}(21)$ & $107(2)$ & & \\
\hline $\mathrm{N}(9)-\mathrm{C}(11)-\mathrm{C}(21)$ & $107(2)$ & & \\
\hline $\mathrm{C}(21) \# 1-\mathrm{C}(11)-\mathrm{C}(21)$ & $87(4)$ & & \\
\hline $\mathrm{N}(8)-\mathrm{N}(11)-\mathrm{N}(6)$ & $106(2)$ & & \\
\hline $\mathrm{C}(11)-\mathrm{C}(21)-\mathrm{C}(21) \# 1$ & $46.6(19)$ & & \\
\hline $\mathrm{C}(11)-\mathrm{C}(21)-\mathrm{C}(21 \mathrm{~A})$ & $43.8(19)$ & & \\
\hline $\mathrm{C}(21) \# 1-\mathrm{C}(21)-\mathrm{C}(21 \mathrm{~A})$ & $89(2)$ & & \\
\hline $\mathrm{C}(5)-\mathrm{C}(5 \mathrm{~A})-\mathrm{C}(7)$ & $74(4)$ & & \\
\hline $\mathrm{C}(5)-\mathrm{C}(5 \mathrm{~A})-\mathrm{N}(6)$ & $52.8(17)$ & & \\
\hline $\mathrm{C}(7)-\mathrm{C}(5 \mathrm{~A})-\mathrm{N}(6)$ & $90(3)$ & & \\
\hline $\mathrm{N}(7)-\mathrm{N}(7 \mathrm{~A})-\mathrm{C}(18)$ & $83(5)$ & & \\
\hline
\end{tabular}


Table 5.4: Anisotropic displacement parameters $\left(\AA^{2} \times 10^{3}\right)$ for 5 . The anisotropic displacement factor exponent takes the form: $-2 \pi^{2}\left[\mathrm{~h}^{2} \mathrm{a}^{* 2} \mathrm{U}^{11}+\ldots+2 \mathrm{~h} \mathrm{k} \mathrm{a*} \mathrm{b}^{*}\right.$ $\left.\mathrm{U}^{12}\right]$.

\begin{tabular}{lcccccc}
\hline & $\mathrm{U}^{11}$ & $\mathrm{U}^{22}$ & $\mathrm{U}^{33}$ & $\mathrm{U}^{23}$ & $\mathrm{U}^{13}$ & $\mathrm{U}^{12}$ \\
& & & & & & \\
\hline $\mathrm{In}(1)$ & $72(1)$ & $80(1)$ & $74(1)$ & 0 & $-2(1)$ & 0 \\
$\mathrm{C}(2)$ & $140(3)$ & $140(3)$ & $141(3)$ & $2(1)$ & $-1(1)$ & $2(1)$ \\
$\mathrm{N}(3)$ & $144(14)$ & $169(16)$ & $120(9)$ & $40(10)$ & $19(7)$ & $-10(10)$ \\
$\mathrm{N}(4)$ & $230(20)$ & $258(14)$ & $310(20)$ & 0 & $-181(18)$ & 0 \\
$\mathrm{C}(5)$ & $188(19)$ & $240(20)$ & $122(12)$ & 0 & $74(11)$ & 0 \\
$\mathrm{~N}(6)$ & $166(13)$ & $140(20)$ & $142(13)$ & $13(10)$ & $76(12)$ & $60(15)$ \\
$\mathrm{N}(7)$ & $219(16)$ & $181(10)$ & $186(13)$ & $-23(11)$ & $-107(13)$ & $-35(7)$ \\
$\mathrm{N}(8)$ & $124(3)$ & $124(3)$ & $124(3)$ & $0(1)$ & $0(1)$ & $1(1)$ \\
$\mathrm{N}(9)$ & $144(17)$ & $168(15)$ & $111(10)$ & 0 & $55(11)$ & 0 \\
$\mathrm{~N}(10)$ & $120(3)$ & $120(3)$ & $119(3)$ & $1(1)$ & $0(1)$ & $0(1)$ \\
$\mathrm{C}(11)$ & $217(17)$ & $240(20)$ & $138(15)$ & 0 & $99(14)$ & 0 \\
$\mathrm{~N}(11)$ & $133(14)$ & $174(17)$ & $153(12)$ & $13(11)$ & $33(11)$ & $66(12)$ \\
$\mathrm{C}(13)$ & $212(9)$ & $212(9)$ & $212(9)$ & $0(1)$ & $0(1)$ & $0(1)$ \\
$\mathrm{C}(21)$ & $190(20)$ & $300(30)$ & $210(20)$ & $50(20)$ & $89(18)$ & $-48(16)$ \\
$\mathrm{C}(5 \mathrm{~A})$ & $210(30)$ & $250(20)$ & $163(14)$ & $-48(17)$ & $106(16)$ & $0(20)$ \\
$\mathrm{N}(7 \mathrm{~A})$ & $164(11)$ & $183(11)$ & $206(14)$ & $-43(8)$ & $-80(11)$ & $-34(8)$ \\
$\mathrm{C}(14)$ & $186(5)$ & $184(5)$ & $186(5)$ & $1(1)$ & $0(1)$ & $0(1)$ \\
$\mathrm{C}(21 \mathrm{~A})$ & $240(20)$ & $280(30)$ & $160(20)$ & $62(17)$ & $77(19)$ & $-30(20)$ \\
$\mathrm{C}(7)$ & $230(30)$ & $290(30)$ & $153(18)$ & $-20(20)$ & $86(17)$ & $-50(20)$ \\
$\mathrm{C}(13 \mathrm{~A})$ & $203(8)$ & $202(8)$ & $202(8)$ & $0(1)$ & $0(1)$ & $0(1)$ \\
$\mathrm{C}(24)$ & $182(5)$ & $183(5)$ & $183(5)$ & $0(1)$ & $-1(1)$ & $0(1)$ \\
$\mathrm{C}(18)$ & $170(10)$ & $221(11)$ & $188(11)$ & $-85(10)$ & $-30(10)$ & $-58(7)$
\end{tabular}


Table 5.5: Hydrogen coordinates $\left(\mathrm{x} 10^{4}\right)$ and isotropic displacement parameters $\left(\AA^{2} \mathrm{x} 10^{3}\right)$ for $\mathbf{5}$.

\begin{tabular}{lcccc}
\hline & $\mathrm{x}$ & $\mathrm{y}$ & $\mathrm{z}$ & $\mathrm{U}(\mathrm{eq})$ \\
& & & & \\
& & & & \\
$\mathrm{H}(13 \mathrm{~A})$ & -694 & 61 & 8054 & 318 \\
$\mathrm{H}(13 \mathrm{~B})$ & -749 & 906 & 8891 & 318 \\
$\mathrm{H}(13 \mathrm{C})$ & -59 & 308 & 9156 & 318 \\
$\mathrm{H}(14 \mathrm{~A})$ & 2181 & -392 & 10911 & 278 \\
$\mathrm{H}(14 \mathrm{~B})$ & 1541 & -46 & 9903 & 278 \\
$\mathrm{H}(14 \mathrm{C})$ & 1561 & 212 & 11527 & 278 \\
$\mathrm{H}(13 \mathrm{D})$ & 495 & -120 & 6370 & 304 \\
$\mathrm{H}(13 \mathrm{E})$ & 936 & 175 & 7742 & 304 \\
$\mathrm{H}(13 \mathrm{~F})$ & 1055 & 638 & 6277 & 304 \\
$\mathrm{H}(24 \mathrm{~A})$ & 3192 & 1670 & 10048 & 275 \\
$\mathrm{H}(24 \mathrm{~B})$ & 3427 & 946 & 11085 & 275 \\
$\mathrm{H}(24 \mathrm{C})$ & 2878 & 1648 & 11624 & 275 \\
& & & & \\
\hline
\end{tabular}

\section{Quantum-Chemical Computations}

Table 5.6: Ligand dissociation enthalpy at STP (298 K, 1 bar) and HOMO/LUMO energy levels for $\operatorname{In}(\text { famd })_{3} 193$ and $\mathbf{5}$.

\begin{tabular}{ccccccc}
\hline & $\begin{array}{c}1 \text { st } \\
(\mathrm{kJ} / \mathrm{mol})\end{array}$ & $\begin{array}{c}2 \mathrm{nd} \\
(\mathrm{kJ} / \mathrm{mol})\end{array}$ & $\begin{array}{c}3 \mathrm{rd} \\
(\mathrm{kJ} / \mathrm{mol})\end{array}$ & $\begin{array}{c}\mathrm{HOMO} \\
(\mathrm{eV})\end{array}$ & $\begin{array}{c}\text { LUMO } \\
(\mathrm{eV})\end{array}$ & $\begin{array}{c}\text { LUMO- } \\
\text { HOMO } \\
(\mathrm{eV})\end{array}$ \\
\hline $\operatorname{In}(\text { famd })_{3}$ & 312 & 382 & 510 & -5.45 & 0.25 & 5.70 \\
$\mathbf{5}$ & 289 & 301 & 570 & -5.90 & -0.69 & 5.21 \\
\hline
\end{tabular}


Table 5.7: Natural bond orbitals charges of the indium metal center and the bonding nitrogen atoms for $\operatorname{In}(\text { form })_{3}$ and $\mathbf{5}$.

\begin{tabular}{cccc}
\hline & Indium & Bonding Nitrogen & Bond \\
\hline $\operatorname{In}(\text { form })_{3}$ & 1.780 & -0.686 & 2.466 \\
$\mathbf{5}$ & 1.471 & -0.440 & 1.911 \\
\hline
\end{tabular}

Table 5.8: Enthalpy for the decomposition paths at 1 bar. All energies are in kJ $\mathrm{mol}^{-1}$ relative to undecomposed $\mathbf{5}$.

\begin{tabular}{ccccccc}
\hline Temperature & $\mathbf{1 - 2}^{\ddagger}$ & $\mathbf{2}$ & $\mathbf{2 - 3 a}^{\ddagger}$ & $\mathbf{3 a}$ & $\mathbf{2 - 3}^{\ddagger}$ & $\mathbf{3 b}$ \\
\hline $200{ }^{\circ} \mathrm{C}$ & 241 & 204 & 370 & 81 & 393 & -63 \\
$250{ }^{\circ} \mathrm{C}$ & 241 & 204 & 369 & 80 & 393 & -63 \\
$300{ }^{\circ} \mathrm{C}$ & 241 & 203 & 369 & 80 & 392 & -64 \\
$350{ }^{\circ} \mathrm{C}$ & 242 & 203 & 368 & 79 & 392 & -65 \\
\hline
\end{tabular}

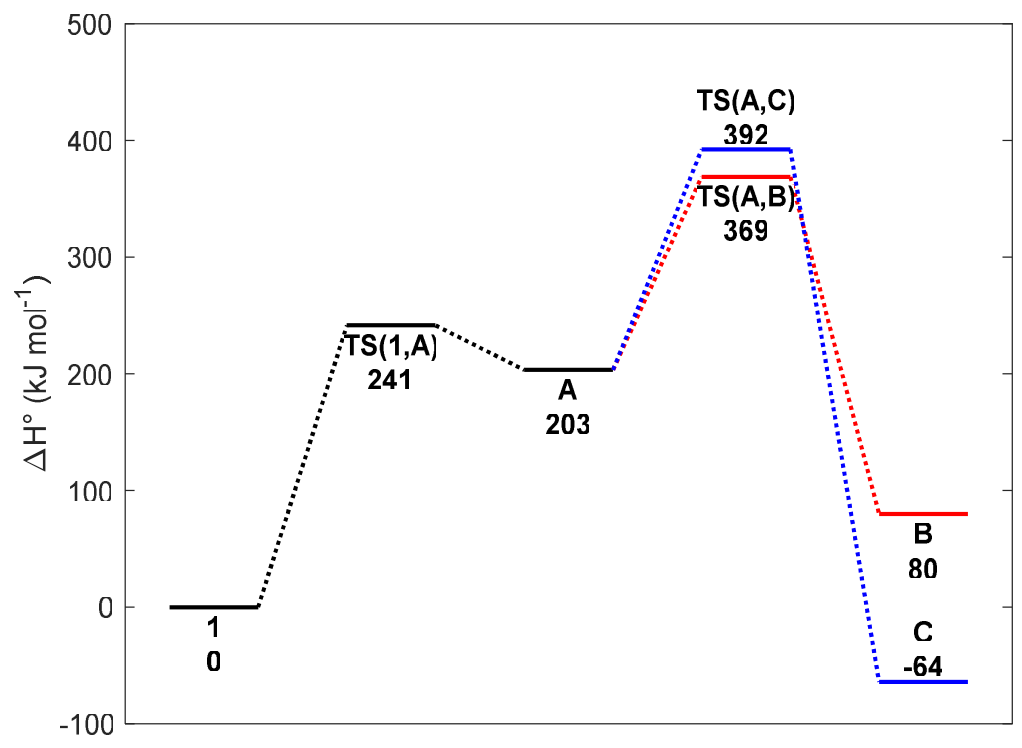

Figure 5.12: Enthalpy at $300{ }^{\circ} \mathrm{C}$ of the decomposition paths. All energies are relative to 5 .

Table 5.9: Free energies for the decomposition paths at 1 bar. All energies are in $\mathrm{kJ} \mathrm{mol}^{-1}$ relative to undecomposed $\mathbf{5}$.

\begin{tabular}{ccccccc}
\hline Temperature & $\mathbf{1 - 2}^{\ddagger}$ & $\mathbf{2}$ & $\mathbf{2 - 3 a}^{\ddagger}$ & $\mathbf{3 a}$ & $\mathbf{2 - 3 b ^ { \ddagger }}$ & $\mathbf{3 b}$ \\
\hline $200{ }^{\circ} \mathrm{C}$ & 241 & 126 & 292 & 5 & 319 & -213 \\
$250{ }^{\circ} \mathrm{C}$ & 241 & 118 & 283 & -3 & 311 & -229 \\
$300{ }^{\circ} \mathrm{C}$ & 241 & 110 & 275 & -11 & 303 & -245 \\
$350{ }^{\circ} \mathrm{C}$ & 241 & 101 & 267 & -19 & 296 & -261 \\
\hline
\end{tabular}


Table 5.10: Reaction enthalpy for the decomposition paths of 5 to a tricoordinated In(III) species at 1 bar. All energies are in $\mathrm{kJ} \mathrm{mol}^{-1}$.

\begin{tabular}{ccccccc}
\hline Temperature & $\begin{array}{c}\text { Path A (1-3a) } \\
\text { 1st Ligand }\end{array}$ & $\begin{array}{c}\text { 2nd } \\
\text { Ligand }\end{array}$ & $\begin{array}{c}\text { 3rd } \\
\text { Ligand }\end{array}$ & $\begin{array}{c}\text { Path B (1-3b) } \\
\text { 1st Ligand }\end{array}$ & $\begin{array}{c}\text { 2nd } \\
\text { Ligand }\end{array}$ & $\begin{array}{c}\text { 3rd } \\
\text { Ligand }\end{array}$ \\
\hline $200{ }^{\circ} \mathrm{C}$ & 81 & 86 & 80 & -63 & -70 & -54 \\
$250{ }^{\circ} \mathrm{C}$ & 80 & 85 & 80 & -63 & -71 & -54 \\
$300{ }^{\circ} \mathrm{C}$ & 80 & 85 & 79 & -64 & -72 & -55 \\
$350{ }^{\circ} \mathrm{C}$ & 79 & 84 & 79 & -65 & -72 & -56 \\
\hline
\end{tabular}

Table 5.11: Reaction free energies for the decomposition paths of $\mathbf{5}$ to a tricoordinated $\operatorname{In}(\mathrm{III})$ species at 1 bar. All energies are in $\mathrm{kJ} \mathrm{mol}^{-1}$.

\begin{tabular}{ccccccc}
\hline Temperature & $\begin{array}{c}\text { Path A (1-3a) } \\
\text { 1st Ligand }\end{array}$ & $\begin{array}{c}\text { 2nd } \\
\text { Ligand }\end{array}$ & $\begin{array}{c}\text { 3rd } \\
\text { Ligand }\end{array}$ & $\begin{array}{c}\text { Path B (1-3b) } \\
\text { 1st Ligand }\end{array}$ & $\begin{array}{c}\text { 2nd } \\
\text { Ligand }\end{array}$ & $\begin{array}{c}\text { 3rd } \\
\text { Ligand }\end{array}$ \\
\hline $200{ }^{\circ} \mathrm{C}$ & 5 & 1 & -2 & -213 & -233 & -202 \\
$250{ }^{\circ} \mathrm{C}$ & -3 & -8 & -11 & -229 & -250 & -217 \\
$300{ }^{\circ} \mathrm{C}$ & -11 & -16 & -20 & -245 & -268 & -233 \\
$350{ }^{\circ} \mathrm{C}$ & -19 & -25 & -29 & -261 & -285 & -248 \\
\hline
\end{tabular}

\section{Growth Behaviour Curve on Si(100)}

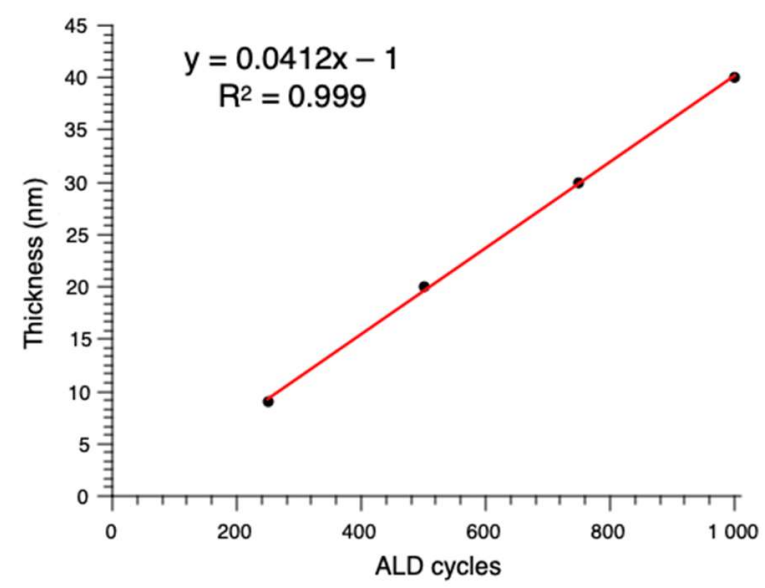

Figure 5.13: Growth behavior of $\mathbf{5}$ at $220{ }^{\circ} \mathrm{C}$ dependent on the number of cycles using a $5 \mathrm{~s}$ pulse of $\mathbf{5}$ and a $9 \mathrm{~s} \mathrm{NH}_{3}$ plasma pulse. The red line indicates the linear trend. 


\section{XRD Analysis of $\operatorname{InN}$ on $\mathrm{Si}(100)$ and $\mathrm{SiC}$}

The intensity of the (0002) grazing incidence X-ray diffraction (GIXRD) peak increased with temperature (Figure 5.14a, 5.14b and 5.14c). The film was analysed

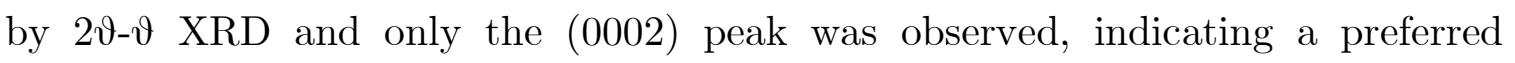
orientation along the c-axis (Figure 5.14d). The presence of the (0002) peak in both

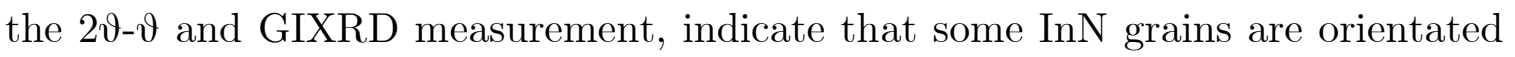
along the c-axis whilst others are titled with respect to the c-axis.
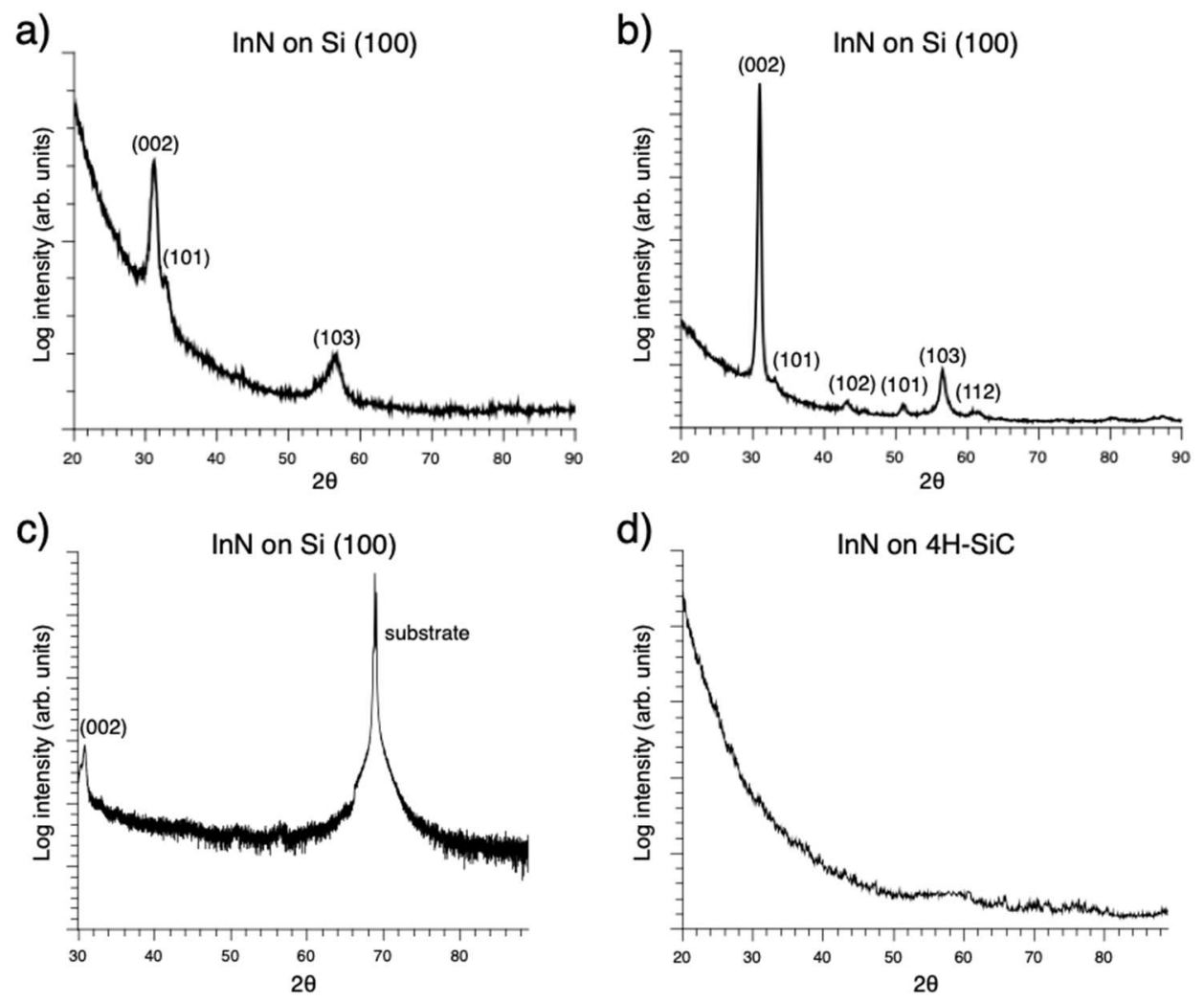

Figure 5.14: a) GIXRD on $\mathrm{Si}(100), 5 \mathrm{~s}$ of $\mathbf{5}$ and $9 \mathrm{~s} \mathrm{NH}_{3}$ plasma pulse deposited at $220{ }^{\circ} \mathrm{C}$ showing crystalline hexagonal wurtzite InN. b) GIXRD on $\mathrm{Si}(100), 5 \mathrm{~s}$ of $\mathbf{5}$ and $9 \mathrm{~s} \mathrm{NH}_{3}$ plasma pulse deposited at $300{ }^{\circ} \mathrm{C}$ showing crystalline hexagonal wurtzite InN. c) $\vartheta-2 \vartheta$ XRD of InN film deposited at $325{ }^{\circ} \mathrm{C}$ with $5 \mathrm{~s}$ of 5 and $9 \mathrm{~s}$ $\mathrm{NH}_{3}$ pulse. d) GIXRD of $\mathrm{InN}$ on $4 \mathrm{H}-\mathrm{SiC}$ substrate deposited at $325{ }^{\circ} \mathrm{C}$ with $5 \mathrm{~s}$ of 5 and $9 \mathrm{~s} \mathrm{NH}_{3}$ pulse. 


\section{Pole Figure Analysis}

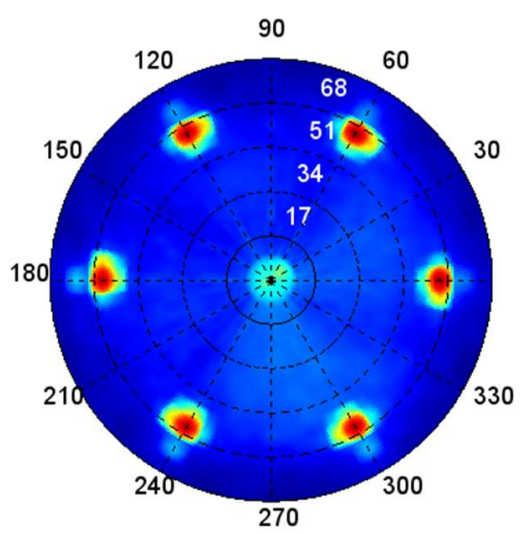

Figure 5.15: Pole figure of the InN (10-11) plane showing 12 poles, with 6 poles corresponding to $4 \mathrm{H}-\mathrm{SiC}$ (hkil) and 6 poles to $\mathrm{InN}(10-11)$. Note that the poles for $4 \mathrm{H}-\mathrm{SiC}$ and $\mathrm{InN}$ overlap. The red color corresponds to $\mathrm{SiC}$ and the green/yellow color corresponds to InN (10-11).

\section{SEM Analysis of InN on $\mathrm{Si}(100)$}

The top-view SEM of the InN films on Si shows InN crystallites for both temperature intervals, in line with the XRD results (Figure 5.16a and 5.16b). The crystal grains for a) seem to have coalesced to form a network structure instead of individual grains as for b). This is expected due to only the temperature increase, which leads to more diffusion on the surface in which smaller grains merge into larger ones. This trend was more noticeable when the deposition temperature was increase to $350{ }^{\circ} \mathrm{C}$ (Figure 5.16c). This was also indicated from the GIXRD results that showed a smaller FWHM for interval II in comparison to interval I. 

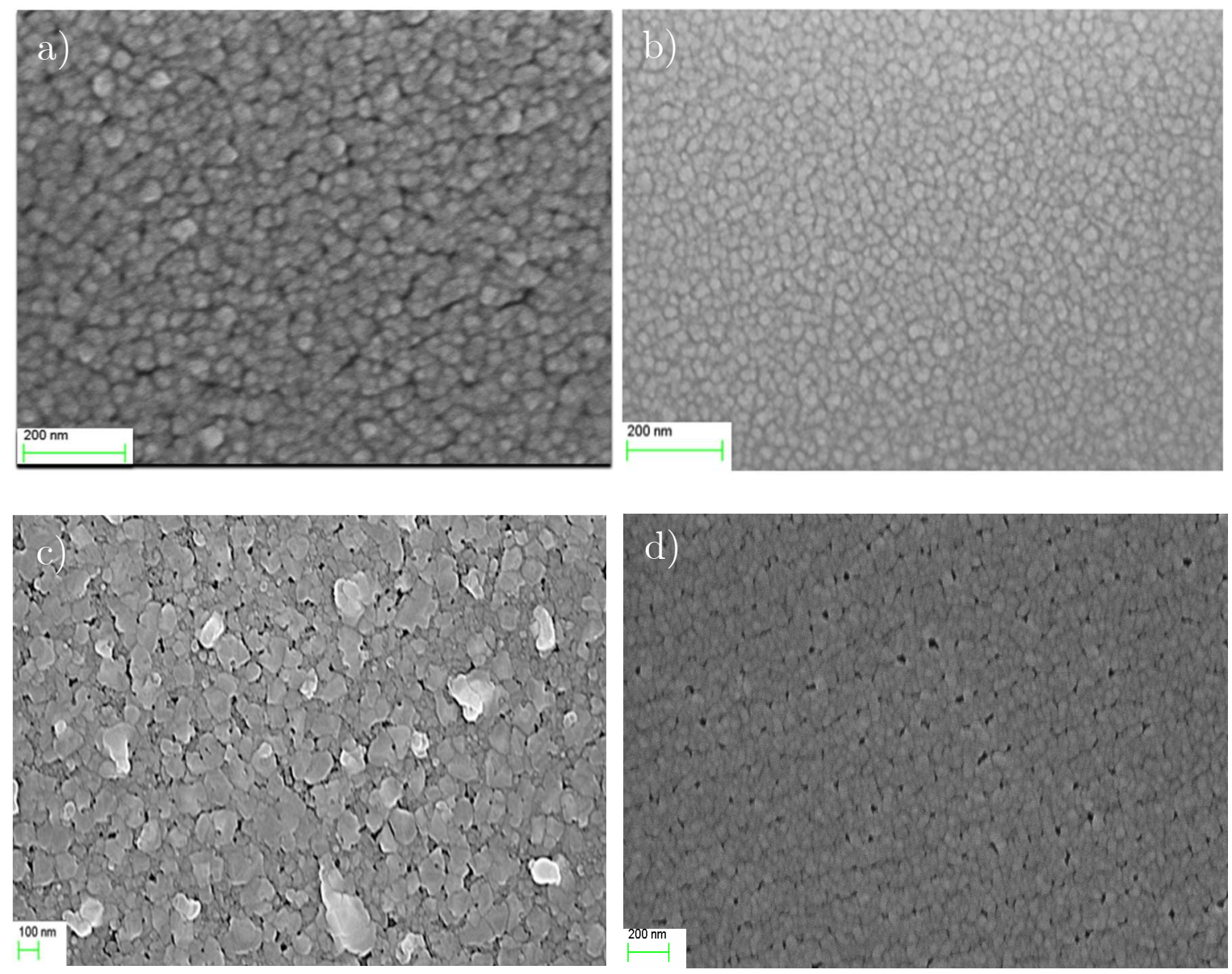

Figure 5.16: Top view SEM of $\mathrm{InN}\left(\mathbf{5}+\mathrm{NH}_{3}\right.$ plasma) on a) Si deposited at $325{ }^{\circ} \mathrm{C}$, b) Si deposited at $220{ }^{\circ} \mathrm{C}$, c) Si deposited at $350{ }^{\circ} \mathrm{C}$ and d) $4 \mathrm{H}-\mathrm{SiC}$ deposited at $325{ }^{\circ} \mathrm{C}$ using high magnification.

\section{X-ray Photoelectron Spectroscopy of InN on $\operatorname{Si}(100)$ and}

\section{H-SiC}

X-ray photoelectron spectroscopy (XPS) of the film deposited on $\operatorname{Si}(100)$ at $325{ }^{\circ} \mathrm{C}$ with $5 \mathrm{~s}$ precursor pulse and $9 \mathrm{~s} \mathrm{NH}_{3}$ plasma pulse showed $48.7 \pm 2.4$ at. \% In, 48.7

\pm 2.4 at. $\% \mathrm{~N}, 2.6 \pm 0.1$ at. $\% \mathrm{O}$ and no detectable $\mathrm{C}$ (Figure 5.17). The same amount of oxygen is bonded to In and $\mathrm{N}$, giving perfect $\mathrm{In} / \mathrm{N}$ ratio of 1.00. It should be noted that the InN film was capped with approximately $15 \mathrm{~nm}$ of AlN in order to avoid post deposition oxidation of the InN film. The high resolution XPS of In 
$3 \mathrm{~d}_{5 / 2}$ and N1s was fitted with two sub-peak each, the major contribution at 444.5 $\mathrm{eV}$ assigned to In- $\mathrm{N}$ bond and a minor contribution at $445.5 \mathrm{eV}$ assigned to In-O bond for In $3 \mathrm{~d}_{5 / 2}$, the major contribution at $396.7 \mathrm{eV}$ was assigned to N-In bond and the minor contribution at $397.7 \mathrm{eV}$ was assigned to $\mathrm{N}-\mathrm{O}$ for $\mathrm{N} 1 \mathrm{~s} .{ }^{225-227}$
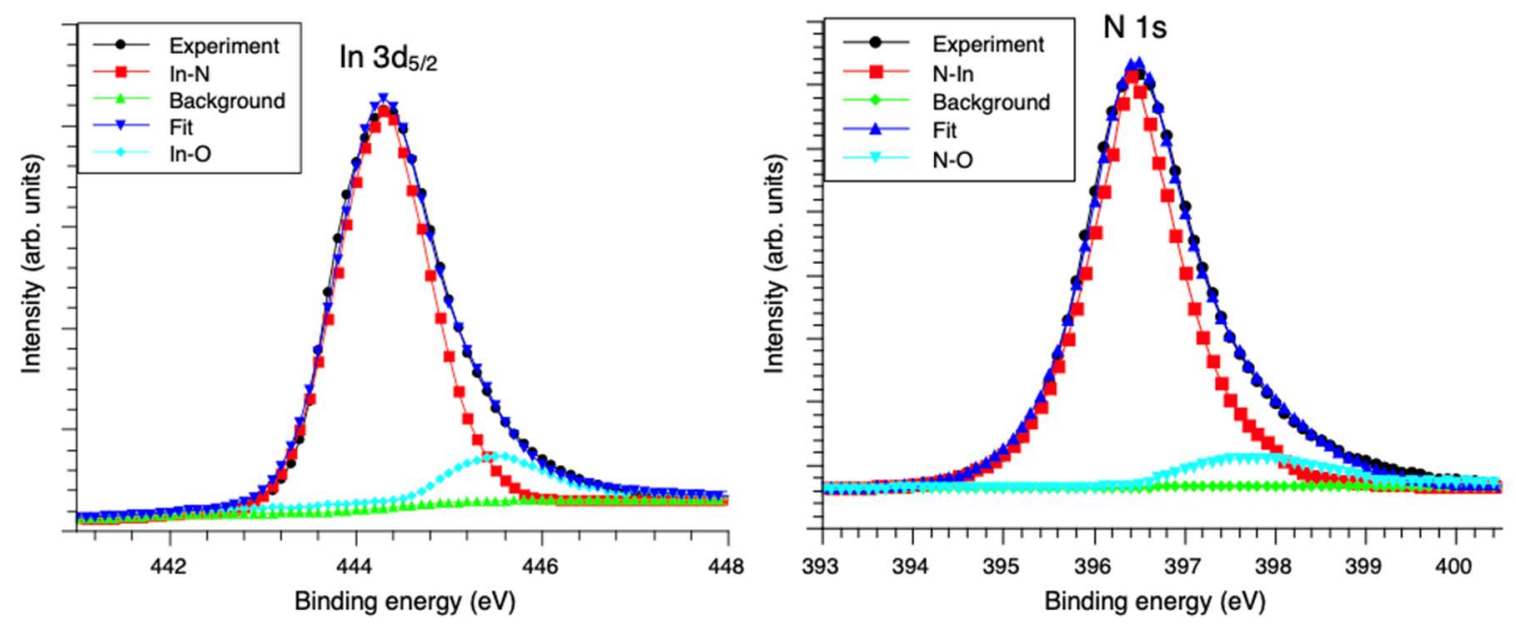

Figure 5.17: High resolution XPS for In $3 \mathrm{~d}_{5 / 2}$ a), and N 1s b), deposited on $\operatorname{Si}(100)$ at $325{ }^{\circ} \mathrm{C}$ with $5 \mathrm{~s}$ pulse of $\mathbf{5}$ and $9 \mathrm{~s}$ pulse of $\mathrm{NH}_{3}$ plasma. The data was fitted with two sub-peaks, which correspond to the $\mathrm{M}-\mathrm{N}$ bond, $\mathrm{In}-\mathrm{O}$ and $\mathrm{N}-\mathrm{O}$ bonds.
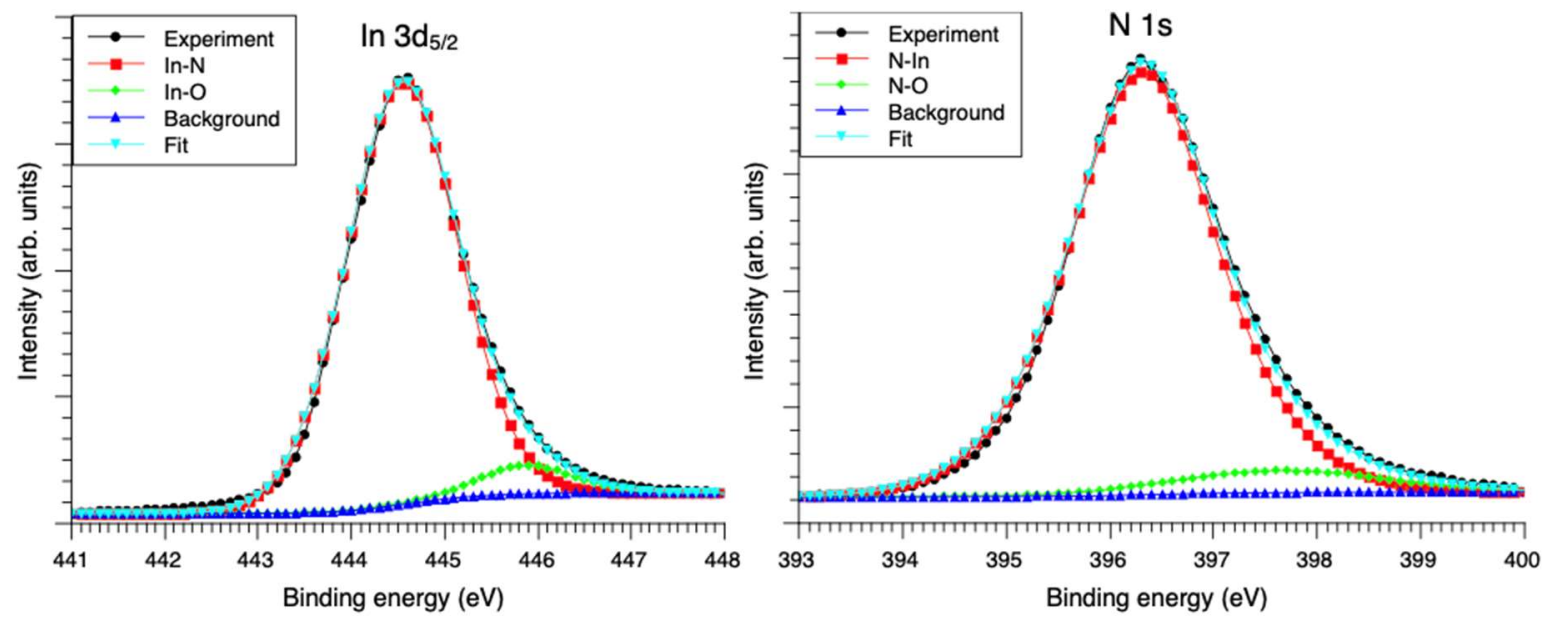

Figure 5.18: High resolution XPS for In $3 \mathrm{~d}_{5 / 2}$ a), and $\mathrm{N} 1 \mathrm{~s} \mathrm{~b}$ ), deposited on $4 \mathrm{H}-\mathrm{SiC}$ at $325{ }^{\circ} \mathrm{C}$ with $5 \mathrm{~s}$ pulse of $\mathbf{5}$ and $9 \mathrm{~s}$ pulse of $\mathrm{NH}_{3}$ plasma. The data was fitted with two sub-peaks, which correspond to the $\mathrm{M}-\mathrm{N}$ bond, $\mathrm{In}-\mathrm{O}$ and $\mathrm{N}-\mathrm{O}$ bonds. 


\section{Raman Spectroscopy Analysis of InN on Si(100)}

Raman spectroscopy of the InN films on Si deposited at $325{ }^{\circ} \mathrm{C}$ shows the $\mathrm{A}_{1}(\mathrm{LO})$ band of wurtzite InN at approximately $580 \mathrm{~cm}^{-1}$ (Figure 5.19). This agrees with the previously reported Raman shift, ${ }^{228}$ indicating low stress in the film. A small shoulder was observed at $375 \mathrm{~cm}^{-1}$, which is a gap mode previously shown to correspond to In vacancies in InN. ${ }^{228}$ The appearance of this gap mode is attributed to the small amount oxygen in the film.

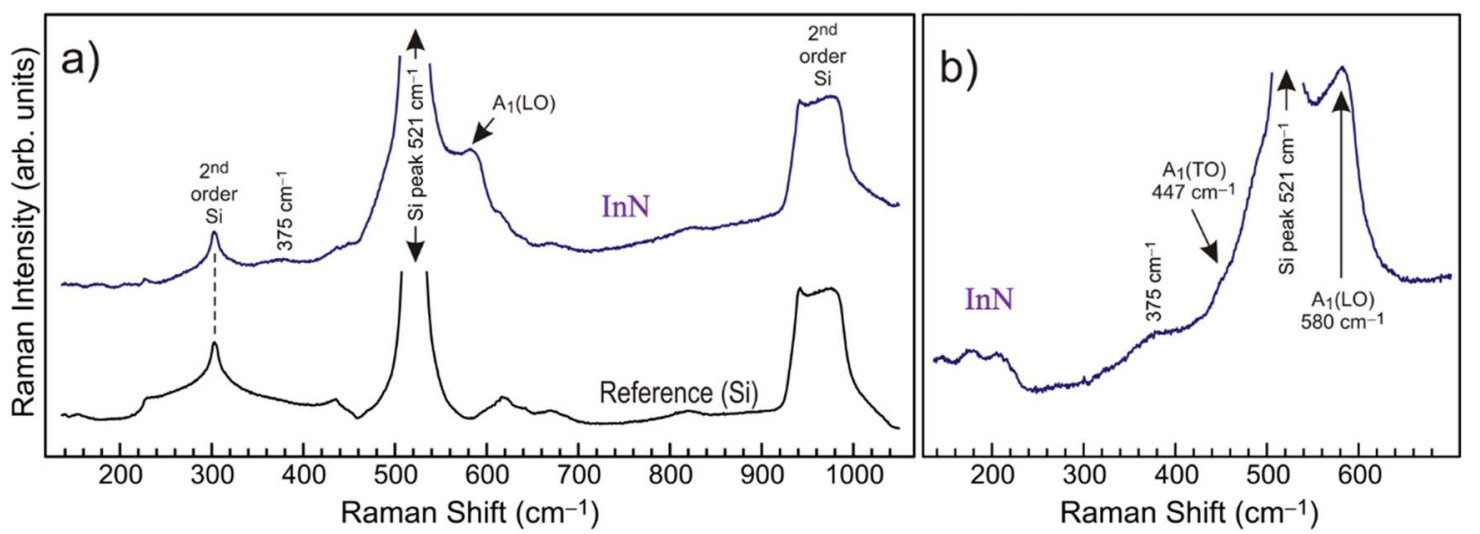

Figure 5.19: a) The Raman spectrum of InN deposited from 5 at $300{ }^{\circ} \mathrm{C}$ on $\mathrm{Si}(100)$ together with the reference spectrum of the Si substrate provided for comparison. b) The spectra of the samples after subtraction of the substrate spectrum. 


\section{Chapter 5 - Part II}

\section{Novel Aluminum MeNacNac Compounds as}

\section{Possible Precursors for Atomic Layer}

\section{Deposition}

A manuscript for the following work is in preparation. Seán T. Barry a and Jason D. Masuda $a^{b}$ are co-contributors to this work.

aDepartment of Chemistry, Carleton University, Ottawa, Ontario K1S 5B6, Canada

bDepartment of Chemistry, St. Mary's University, Halifax, Nova Scotia B3H 3C3, Canada 


\subsubsection{Abstract}

Three aluminum ${ }^{M e N a c N a c}$ species were synthesized and investigated as potential precursors for aluminum oxide and aluminum nitride atomic layer deposition (ALD). Compound 6 is a novel $\mathrm{Al}\left(\mathrm{Me}^{\mathrm{NacNac}}\right)\left(\mathrm{NMe}_{2}\right)_{2}$ species that is thermally stable and volatile by thermogravimetric analysis (TGA) with a 1 Torr temperature of $74{ }^{\circ} \mathrm{C}$. It also produced an interesting oxo-bridged structure when exposed to atmosphere, demonstrating the usefulness of its protecting and anchoring ligands. Compound 7 ( $\left.\mathrm{Al}\left({ }^{\mathrm{MeNacNac}}\right) \mathrm{H}_{2}\right)$ has been previously reported, but was demonstrated by TGA to be thermally unstable at elevated temperatures and therefore unsuitable for use as an ALD precursor. Its decomposition was studied by NMR tube thermolysis. Finally, compound $\mathbf{8}$ is the first report of a reduced MeNacNac ligand in the species $\mathrm{Al}\left(\mathrm{MeNacNac}^{\mathrm{r}, \mathrm{Me}} \mathrm{NacNac}\right)$, i.e. $\mathrm{Al}\left({ }^{\mathrm{MeNacNac}}\right)\left(\left(\mathrm{NCH}_{3}\right) \mathrm{CH}\left(\mathrm{CH}_{3}\right) \mathrm{CHC}\left(\mathrm{CH}_{3}\right)\left(\mathrm{NCH}_{3}\right)\right)$. Its thermal stability and aluminum-nitrogen bonds make it useful as a potential AlN ALD precursor, though its higher 1 Torr temperature of $131{ }^{\circ} \mathrm{C}$ compared to 6 indicates a reduced volatility. 


\subsubsection{Introduction}

Both aluminum oxide and aluminum nitride are useful microelectronic materials. Aluminum oxide has a large band gap of $9 \mathrm{eV}$, a high dielectric constant, and high chemical and thermal stability; for this reason, it is extremely useful to use as a dielectric of passivation layer. ${ }^{24}$ Aluminum nitride can also be used as a dielectric layer with its band gap of $6.2 \mathrm{eV}$, and can be used as a component in building light-emitting diodes (LEDs). ${ }^{1}$ AlN is beneficial for use in microelectronic devices because it self-passivates in high-oxidizing and high-temperature environments, in addition to being inert to chemical attack. ${ }^{3}$ For modern microelectronic applications, atomic layer deposition (ALD) is a powerful technique to employ due to its self-terminating and surface-controlled chemical reactions that allow for precise thickness and conformality control on small substrates with nontrivial geometries. Trimethylaluminum (TMA) has been widely used as a popular precursor for the deposition of aluminum oxide and aluminum nitride by ALD, though its drawbacks highlighted below allow for significant improvements to be made in this realm..$^{25}$

This work investigates the synthesis and characterization of novel aluminum $\beta$-diketiminate compounds that could be used as TMA-replacements for aluminum oxide and aluminum nitride deposition by ALD. Films deposited using TMA often suffer from carbon contamination on the order of $3-6 \% .37,97,100$ The NacNac 
framework was employed in order to introduce metal-nitrogen bonds in the precursor and prevent having direct bonds between aluminum and carbon. We hypothesize that this could reduce carbon contamination in films, which has been known to occur do the presence of direct metal-carbon bonds in TMA. ${ }^{37}$ Previously, we have reported an amide analogue of TMA that was shown to reduce carbon contamination in aluminum oxide films deposited by ALD to less than $1 \%{ }^{75}$ Previous publications have reported using $\beta$-diketonates for metal oxide vapour depositions, including acetylacetonate ligands to deposit cobalt oxide thin films by chemical vapour deposition (CVD) ${ }^{65}$ Because aluminum is so oxophilic, it is imperative to keep aluminum-oxygen bonds out of potential AlN precursors; this would undoubtably lead to oxygen incorporated into deposited films. These $\beta$ diketonate precursors have been modified by replacing one bonding oxygen with a nitrogen atom to produce $\beta$-ketoiminates which have been used to deposit a variety of metal oxides. ${ }^{66}$ Replacing the second bonding atom with a nitrogen atom produces $\beta$-diketiminate ligands that then would only employ metal-nitrogen bonds in a subsequent aluminum precursor.

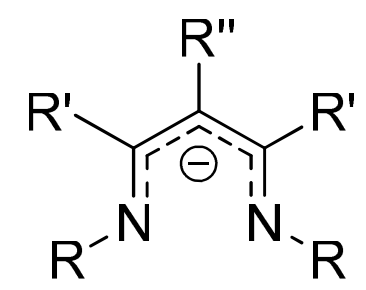

Figure 5.20: General structure of a NacNac ligand. For ${ }^{\mathrm{MeNacNac}}, \mathrm{R}=\mathrm{R}$ ' $=\mathrm{CH}_{3}$ and $\mathrm{R} "=\mathrm{H}$. 
$\beta$-diketiminates, also called NacNacs, are nitrogen-donating ligands that can be employed on a variety of metal centres. These ligands are monoanionic and their chemistry can be tuned according to desired applications through the choice of $\mathrm{R}$ and R' groups on the alpha and beta nitrogen and carbon positions, respectively. ${ }^{229}$ Substitution of the gamma-carbon position is also possible, though this position $(\mathrm{R}$ ") is often protonated. More so, it is intended in the design of possible precursors to employ the larger, stabilizing MeNacNac ligand to protect the $\mathrm{Al}$ centre while maintaining a lightweight compound by using other small heteroleptic ligands to satisfy the coordination of aluminum. Described herein is the synthesis and characterization of a ${ }^{\mathrm{Me}} \mathrm{NacNac}$ aluminum hyride, $\mathrm{Al}\left({ }^{\mathrm{Me}} \mathrm{NacNac}\right) \mathrm{H}_{2}(\mathbf{7})$, as well as two novel aluminum MeNacNac compounds, $\mathrm{Al}\left(\mathrm{MeNacNac}^{\mathrm{M}}\left(\mathrm{NMe}_{2}\right)_{2}\right.$ (6), and $\mathrm{Al}\left({ }^{\mathrm{MeNacNac}}\right)\left({ }^{\mathrm{r}, \mathrm{Me}} \mathrm{NacNac}\right)$ or $\mathrm{Al}\left({ }^{\mathrm{MeNacNac}}\right)\left(\left(\mathrm{NCH}_{3}\right) \mathrm{CH}\left(\mathrm{CH}_{3}\right) \mathrm{CHC}\left(\mathrm{CH}_{3}\right)\left(\mathrm{NCH}_{3}\right)\right)$. (8), where $\mathbf{8}$ shows the first example of a reduced ${ }^{M e N a c N a c ~ l i g a n d . ~}$

\subsubsection{Experimental}

All syntheses were performed air-free, either on a nitrogen Schlenk line or in an MBRAUN Labmaster 130 nitrogen glovebox, unless otherwise specified. Reagents were used as purchased from Sigma Aldrich, and solvents were purchased from VWR and purified in an MBRAUN Manual Solvent Purification system. 


\section{Precursor Synthesis}

\section{$\mathrm{Al}\left({ }^{\mathrm{MeNacNac}}\right)\left(\mathrm{NMe}_{2}\right)_{2}(6)$}

The synthesis for ${ }^{\mathrm{Me}} \mathrm{NacNac}$ was adapted from the process detailed in Stalzer et $a l .{ }^{230}$ and all ligands' NMR spectra were in agreement with the literature.

N-methyl-4-amino-3-penten-2-one - (MeNacac) Toluene (100 mL) was added in air to a $200 \mathrm{~mL}$ round-bottomed flask with a stir bar and was cooled to $0{ }^{\circ} \mathrm{C}$. To this flask, acetylacetone $(20 \mathrm{~mL}, 196 \mathrm{mmol})$ was added, followed by methylamine (18 mL, $40 \mathrm{wt} \%$ in $\mathrm{H}_{2} \mathrm{O}, 209 \mathrm{mmol}$ ). The biphasic solution began clear and colourless and became a bright highlighter yellow with a visible precipitate. The bottom phase was clear yellow while the top contained a crystalline solid. The biphasic solution stirred overnight while warming to room temperature. The following day, the organic phase was separated and stirred with magnesium sulphate. The mixture was filtered, its volume was reduced under vacuum, and the resultant solution was stored in the refrigerator to produce clear and colourless prismatic needle crystals (14.943 g, 68\% yield).

N-N'-dimethyl-4-amino-3-penten-2-immonium tetrafluoroborate $\left(\left[{ }^{\mathrm{MeNacNacH}}\right]\left[\mathrm{BF}_{4}\right]\right)-\mathrm{Et}_{3} \mathrm{O}^{+} \mathrm{BF}_{4}^{-}(5.473 \mathrm{~g}, 28 \mathrm{mmol})$ was dissolved in approximately $30 \mathrm{~mL}$ of dichloromethane (DCM) in a Schlenk flask in the glovebox and was capped with a rubber septum. In air, MeNacac $(3.2 \mathrm{~g}, 28 \mathrm{mmol})$ was dissolved in $30 \mathrm{~mL}$ DCM. The $\mathrm{Et}_{3} \mathrm{O}^{+} \mathrm{BF}_{4}^{-}$solution was placed under $\mathrm{Ar}(\mathrm{g})$ on the 
Schlenk line and was transferred via cannula to the MeNacac solution while stirring with a magnetic stir bar. The clear and colourless solution became clear and light rose in colour. The stirring was stopped, and the solution was left to sit under $\operatorname{Ar}(\mathrm{g})$ for 30 mins. Methylamine $(3.6 \mathrm{~mL}, 28 \mathrm{mmol}, 33 \mathrm{wt} \%$ in $\mathrm{EtOH})$ was added dropwise by syringe and the solution evolved through a clear light yellow, a cloudy light yellow, and finally to a clear bright yellow colour. Volatiles were removed under vacuum and the solution was filtered and placed in the freezer at $-35{ }^{\circ} \mathrm{C}$ to recrystallize. Crystals were washed with hexanes and dried under vacuum (4.275 g, $71 \%$ yield).

N-N'-dimethyl-4-amino-3-penten-2-imine ( ${ }^{\mathrm{MeNacNac}}$ ) - $\left[\mathrm{MeNacNacH}^{\mathrm{Ne}}\left[\mathrm{BF}_{4}\right]\right.$ (9.5 g, $44 \mathrm{mmol}$ ) was dissolved in $70 \mathrm{~mL}$ of anhydrous methanol in a roundbottomed flask. Sodium (1.077 g, $47 \mathrm{mmol})$ was added to a vial containing $20 \mathrm{~mL}$ methanol to produce a slightly cloudy suspension. This NaOMe solution was added to the $\left[{ }^{\mathrm{Me}} \mathrm{NacNacH}\right]\left[\mathrm{BF}_{4}\right]$ solution by pipette and the cloudy suspension was allowed to stir for $1 \mathrm{~h}$ and was left under $\operatorname{Ar}(\mathrm{g})$ overnight. Methanol was removed by vacuum on a Schlenk line to produce a light yellow slush; $70 \mathrm{~mL}$ of toluene was added and the mixture was filtered to produce a clear light yellow solution. The solid was washed with $2 \times 25 \mathrm{~mL}$ toluene and filtered, and the toluene fractions were combined and dried under vacuum to produce a white-yellow solid (4.008 g, $72 \%$ unpurified yield). 
$\mathrm{Al}\left({ }^{\mathrm{MeNacNac}}\right)\left(\mathrm{NMe}_{2}\right)_{2}(\mathbf{6})-\mathrm{AlH}\left(\mathrm{NMe}_{2}\right)_{2}(0.317 \mathrm{~g}, 3 \mathrm{mmol})$ was added to a 50 $\mathrm{mL}$ Schlenk flask with a stir bar and was dissolved in $10 \mathrm{~mL}$ toluene. ${ }^{\mathrm{Me}} \mathrm{NacNac}$ (0.342 $\mathrm{g}, 27 \mathrm{mmol}$ ) was dissolved in $10 \mathrm{~mL}$ toluene in a vial and was added by pipette to the $\mathrm{AlH}\left(\mathrm{NMe}_{2}\right)_{2}$ solution. Hydrogen gas bubbles were observed and evolved to produce a clear yellow solution that was left to stir overnight. Volatiles were removed under vacuum and the concentrated solution was placed in the freezer to produce a solid that melts at room temperature to a yellow-orange oily liquid $(0.259 \mathrm{~g}, \quad 40 \%$ yield $) .{ }^{1} \mathrm{H} \quad \mathrm{NMR} \quad\left(300 \mathrm{MHz}, \mathrm{C}_{6} \mathrm{D}_{6}\right): \delta \quad 1.49 \quad(\mathrm{~s}, \quad 6 \mathrm{H}$, $\left.\mathrm{Al}\left(\mathrm{NCH}_{3}\right) \mathrm{C}\left(\mathrm{CH}_{3}\right) \mathrm{CHC}\left(\mathrm{CH}_{3}\right)\left(\mathrm{NCH}_{3}\right)\right), \quad 2.73 \quad$ (s, $\quad 6 \mathrm{H}$, $\left.\mathrm{Al}\left(\mathrm{NCH}_{3}\right) \mathrm{C}\left(\mathrm{CH}_{3}\right) \mathrm{CHC}\left(\mathrm{CH}_{3}\right)\left(\mathrm{NCH}_{3}\right)\right), 2.95\left(\mathrm{~s}, 12 \mathrm{H}, \mathrm{Al}\left(\mathrm{N}\left(\mathrm{CH}_{3}\right)_{2}\right)_{2}\right), 4.52(\mathrm{~s}, 1 \mathrm{H}$, $\left.\mathrm{Al}\left(\mathrm{NCH}_{3}\right) \mathrm{C}\left(\mathrm{CH}_{3}\right) \mathrm{CHC}\left(\mathrm{CH}_{3}\right)\left(\mathrm{NCH}_{3}\right)\right)$.

\section{$\mathrm{Al}\left({ }^{\mathrm{Me}} \mathrm{NacNac}\right) \mathrm{H}_{2}(7)$}

${ }^{\mathrm{MeNacNac}}(0.358 \mathrm{~g}, 3 \mathrm{mmol})$ was dissolved in $8 \mathrm{~mL}$ toluene in a vial while $\mathrm{AlH}_{3} \mathrm{NMe}_{2} \mathrm{Et}(5.7 \mathrm{~mL}, 0.5 \mathrm{M}$ in hexanes, $3 \mathrm{mmol})$ was added to a $50 \mathrm{~mL}$ Schlenk flask, and both solutions were placed in the $-35{ }^{\circ} \mathrm{C}$ freezer. The ${ }^{\mathrm{Me}} \mathrm{NacNac}$ solution was added to the alane solution; $\mathrm{H}_{2}(\mathrm{~g})$ evolution was observed and a clear, pale yellow solution was produced. Once gas evolution ceased, solvent was removed under vacuum to produce a concentrated liquid that was placed in the freezer. Clear colourless crystals were recovered (0.301 g, yield 69\%). 


\section{$\mathrm{Al}\left({ }^{\mathrm{MeNacNac}}\right)\left({ }^{\mathrm{r}, \mathrm{Me}} \mathrm{NacNac}\right)(8)$}

Method A: MeNacNac $(0.474 \mathrm{~g}, 4 \mathrm{mmol})$ was dissolved in $10 \mathrm{~mL}$ of diethyl ether and $10 \mathrm{~mL}$ of toluene and cooled to $-35{ }^{\circ} \mathrm{C}$. To the ${ }^{\mathrm{MeNacNac}}$ solution, $\mathrm{AlH}_{3} \cdot \mathrm{NMe}_{2} \mathrm{Et}$ (2.5 mL, 0.5 M in hexanes, $1 \mathrm{mmol}$ ) was added dropwise via syringe. Hydrogen gas evolution was observed. A clear yellow solution was obtained that was concentrated under vacuum and placed in the $-35{ }^{\circ} \mathrm{C}$ freezer. Needle crystals were isolated (yield

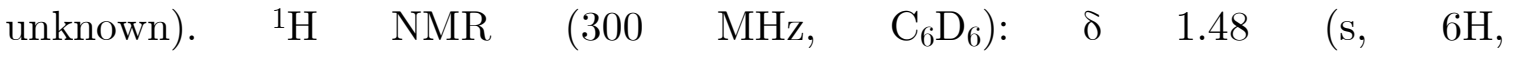
$\left.\mathrm{Al}\left(\mathrm{NCH}_{3}\right) \mathrm{C}\left(\mathrm{CH}_{3}\right) \mathrm{CHC}\left(\mathrm{CH}_{3}\right)\left(\mathrm{NCH}_{3}\right)\right), \quad 1.59 \quad$ (d, $\quad 3 \mathrm{H}$, $\left.\mathrm{Al}\left(\mathrm{NCH}_{3}\right) \mathrm{CH}\left(\mathrm{CH}_{3}\right) \mathrm{CHC}\left(\mathrm{CH}_{3}\right)\left(\mathrm{NCH}_{3}\right)\right), \quad 2.13 \quad$ s, $\quad 3 \mathrm{H}$, $\left.\mathrm{Al}\left(\mathrm{NCH}_{3}\right) \mathrm{CH}\left(\mathrm{CH}_{3}\right) \mathrm{CHC}\left(\mathrm{CH}_{3}\right)\left(\mathrm{NCH}_{3}\right)\right), \quad 2.77 \quad$ (d, $\quad 6 \mathrm{H}$, $\left.\mathrm{Al}\left(\mathrm{NCH}_{3}\right) \mathrm{CH}\left(\mathrm{CH}_{3}\right) \mathrm{CHC}\left(\mathrm{CH}_{3}\right)\left(\mathrm{NCH}_{3}\right)\right), \quad 2.80 \quad(\mathrm{~s}, \quad 6 \mathrm{H}$, $\left.\mathrm{Al}\left(\mathrm{NCH}_{3}\right) \mathrm{C}\left(\mathrm{CH}_{3}\right) \mathrm{CHC}\left(\mathrm{CH}_{3}\right)\left(\mathrm{NCH}_{3}\right)\right), \quad 3.99 \quad(\mathrm{q}, \quad 1 \mathrm{H}$, $\left.\mathrm{Al}\left(\mathrm{NCH}_{3}\right) \mathrm{CH}\left(\mathrm{CH}_{3}\right) \mathrm{CHC}\left(\mathrm{CH}_{3}\right)\left(\mathrm{NCH}_{3}\right)\right), \quad 4.45 \quad$ (d, $\quad 1 \mathrm{H}$, $\left.\mathrm{Al}\left(\mathrm{NCH}_{3}\right) \mathrm{CH}\left(\mathrm{CH}_{3}\right) \mathrm{CHC}\left(\mathrm{CH}_{3}\right)\left(\mathrm{NCH}_{3}\right)\right), \quad 4.56 \quad(\mathrm{~s}, \quad 1 \mathrm{H}$, $\left.\mathrm{Al}\left(\mathrm{NCH}_{3}\right) \mathrm{C}\left(\mathrm{CH}_{3}\right) \mathrm{CHC}\left(\mathrm{CH}_{3}\right)\left(\mathrm{NCH}_{3}\right)\right)$.

Method B: $\mathrm{AlH}_{2}\left({ }^{\mathrm{MeNacNac}}\right)(0.301 \mathrm{~g}, 2 \mathrm{mmol})$ was dissolved in toluene in a 50 $\mathrm{mL}$ Schlenk flask while ${ }^{\mathrm{MeNacNac}}(0.246,2 \mathrm{mmol})$ was dissolved in $10 \mathrm{~mL}$ toluene in a vial. Both were cooled to $-35{ }^{\circ} \mathrm{C}$ and the ${ }^{\mathrm{MeNacNac}}$ solution was subsequently added dropwise by pipette to the $\mathrm{AlH}_{2}\left({ }^{\mathrm{Me}} \mathrm{NacNac}\right)$ solution. The resultant mixture was stirred, bubbled $\mathrm{H}_{2}(\mathrm{~g})$, and became clear yellow. The solution was left to stir 
overnight and was then dried down to a small amount of orange solid (yield unknown).

\section{Thermogravimetric Analysis}

Thermogravimetric analysis (TGA) experiments were performed on a Q500 tool in a nitrogen glovebox. Ramp experiments loaded approximately $10 \mathrm{mg}$ of precursor on a tared platinum pan, and the furnace was heated at a rate of $10{ }^{\circ} \mathrm{C} / \mathrm{min}$ to 500 ${ }^{\circ} \mathrm{C}$ under a flow of 60 sccm $\mathrm{N}_{2}$ (99.998\%). The "1 Torr" temperatures were calculated using mass loss derivative data. ${ }^{20}$

\section{NMR Tube Thermolysis}

Decomposition studies of compound 7 were performed in a typical NMR thermolysis experiment. A sample of 7 was added to a thick-walled NMR tube inside the glovebox and was then dissolved in $\mathrm{d}_{6}$-benzene under an atmosphere of nitrogen on a Schlenk line. The NMR tube was then flame sealed under vacuum on a Schlenk line under an atmosphere of argon gas. The sealed NMR tube was placed in a closed oven and heated. The NMR tube was periodically removed and allowed to cool so that spectra could be collected.

\section{X-ray Crystallography}

Crystals of compounds $\mathbf{6}$ and $\mathbf{8}$ were mounted from Paratone-N oil on an appropriately sized MiTeGen MicroMount. The data were collected on a Bruker APEX II charge-coupled-device (CCD) diffractometer, with an Oxford 700 
Cryocool sample cooling device. The instrument was equipped with graphitemonochromated Mo K $\alpha$ radiation $\left(\lambda=0.71073 \mathrm{~A}^{\circ} ; 30 \mathrm{~mA}, 50 \mathrm{mV}\right)$, with MonoCap X-ray source optics. For data collection, four $\omega$-scan frame series were collected with $0.5^{\circ}$ wide scans, 30 second frames and 366 frames per series at varying $\varphi$ angles $\left(\varphi=0^{\circ}, 90^{\circ}, 180^{\circ}, 270^{\circ}\right)$. Data collection, unit cell refinement, data processing and multi-scan absorption correction were applied using the APEX2 ${ }^{231}$ or APEX3 $3^{232}$ software packages. The structures were solved using SHELXT ${ }^{233}$ and all nonhydrogen atoms were refined anisotropically with SHELXL ${ }^{234}$ using a combination of shelXle ${ }^{235}$ and OLEX2 ${ }^{236}$ graphical user interfaces. Unless otherwise noted, all hydrogen atom positions were idealized and ride on the atom to which they were attached. The final refinement included anisotropic temperature factors on all nonhydrogen atoms. Details of crystal data, data collection, and structure refinement are listed in Table 5.12. All figures were made using ORTEP-3 for Windows. ${ }^{237}$

Compound $\mathbf{8}$ was found to exhibit two site disorder and was refined with a 50:50 ratio. Hydrogen atom $\mathrm{H} 8 \mathrm{~A}$ was fixed at a distance of $0.96 \AA$ from $\mathrm{C} 8 \mathrm{~A}$ and fixed at 1.5 Ueq of $\mathrm{C} 8 \mathrm{~A}$. To give a reasonable model, nitrogen atoms N2A and N2B were forced to have equivalent displacement parameters by using the EADP command. During refinement of $\mathbf{6}$, possible twinning was checked using

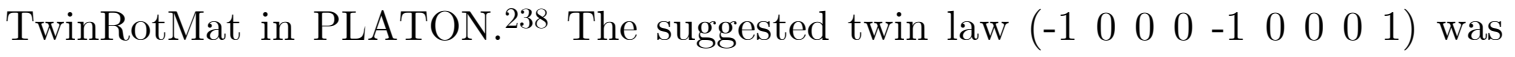


tested and refined to give a BASF value of 0 . As such, this twin law was removed, and the refinement was finalized.

\subsubsection{Results and discussion}

The thermal behaviour of compound $\mathbf{6}$ was evaluated by TGA, shown in Figure 5.21a. The ramp curve shows an exponential mass loss with no visible features in the derivative curve, indicating that $\mathbf{6}$ is a volatile and thermally stable compound. The calculated temperature to produce 1 Torr of vapour pressure from compound 6 is $74{ }^{\circ} \mathrm{C} .{ }^{20}$ It is possible that the thermal stability of $\mathbf{6}$ is enabled by lone pair donation from the imine nitrogen to aluminum's empty p-orbital, while the high volatility at relatively high molecular weight can be attributed to the prevention

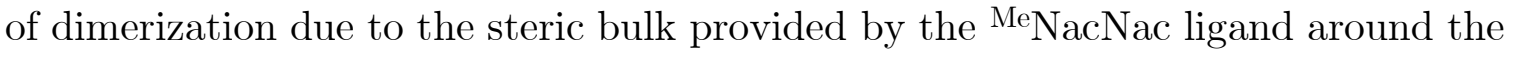
Al centre.
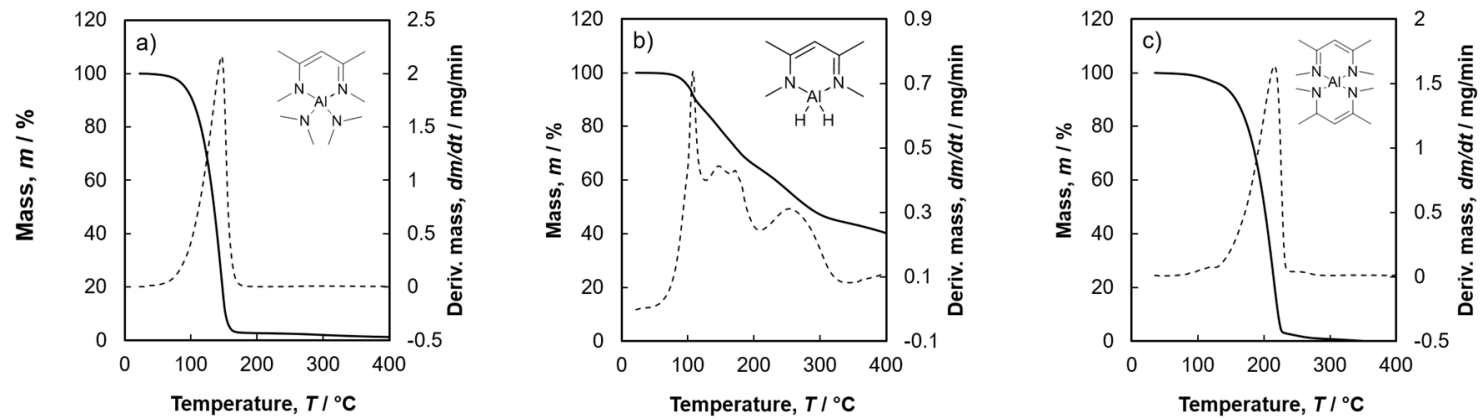

Figure 5.21: Ramp TGA data for compounds a) 6, b) 7, and c) 8 . 
When structural determination of $\mathbf{6}$ was attempted by single crystal X-ray diffraction, an interesting, related structure was obtained instead (Figure 5.22). It is probable that compound $\mathbf{6}$ is air- and moisture-sensitive (unsurprising for a monomeric aluminum species with direct $\mathrm{Al}-\mathrm{N}$ bonds) and 6 was likely exposed to the atmosphere before the crystal structure was obtained. What was produced was an oxo-bridged species between two $\mathrm{Al}$ centres which were also each bonded to one MeNacNac ligand and one $\mathrm{NMe}_{2}$ moiety. While this is not the compound we intended to obtain a crystal structure for, it demonstrates interesting and important information: when exposed to atmosphere, compound 6 eliminates an amine ligand. This means that the heterolepticity of the compound is guiding reactivity the way it is intended to - the stabilizing ${ }^{M e N a c N a c}$ protecting ligand remains bound to the $\mathrm{Al}$ centre while two smaller "anchoring" ligands $\left(\mathrm{NMe}_{2}\right)$ are more readily lost. This is desirable for a potential ALD precursor, particularly in addition to the fact that $\mathbf{6}$ is also volatile, thermally stable, and possesses direct metal-nitrogen bonds. 


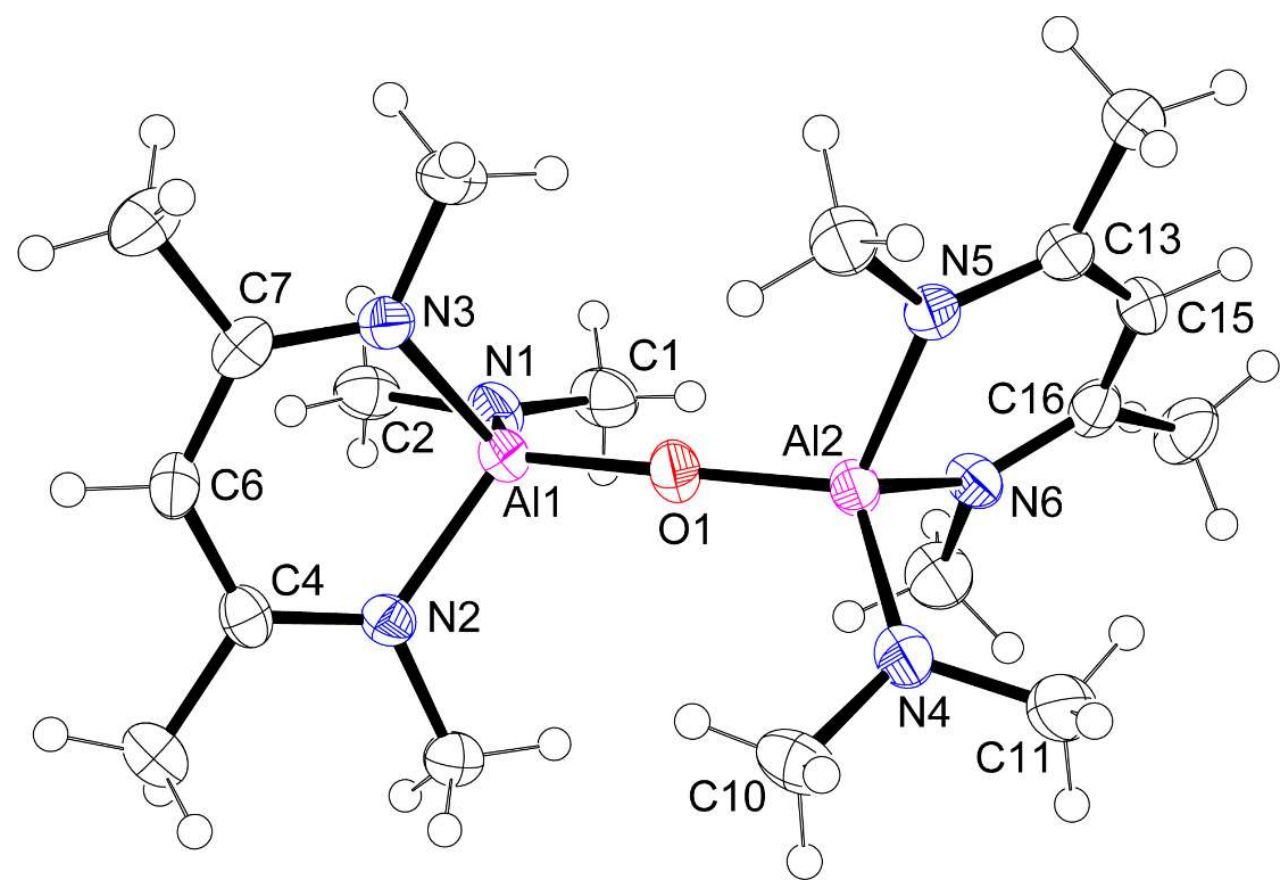

Figure 5.22: Crystal structure for oxo-bridged species obtained after exposing 6 to atmosphere.

Compound 7 was synthesized in an effort to introduce hydride ligands to the precursor: these ligands could provide a reducing "environment" during the deposition of AlN by ALD. Due to the high oxophilicity of aluminum, it is paramount to minimize all possibility of oxygen incorporation during AlN film growth. Hydrogen gas can be used as a separate reducing agent in ALD processes, however, a high energy of over $400 \mathrm{~kJ} / \mathrm{mol}$ is needed to activate this bond ${ }^{76}$ and therefore higher deposition temperatures are required; this is generally undesirable due to resultant substrate limitations. Hydride ligands have been shown in the literature to react with and reduce metal species on a growing film's surface 239 and could, in the case of these precursors, react with any oxygen-containing species to prevent their direct reaction with aluminum. While the intent was to use $\mathbf{7}$ as an 
AlN precursor, TGA data (Figure 5.21b) demonstrated that this would not be viable since the compound is thermally unstable and decomposes readily upon heating. It is possible in this case that the bulky ${ }^{\mathrm{Me}} \mathrm{NacNac}$ ligand was effective in preventing oligomerization, but that this feature allowed the $\mathrm{Al}$ centre to be insufficiently stabilized by the two remaining hydride ligands (as opposed to by two dimethylamide ligands in 6 ). After heating to $100{ }^{\circ} \mathrm{C}$ over $1 \mathrm{~h}$ during the NMR boilup of compound $\mathbf{7}$, this decomposition was observed likely due to a hydrogen evolution. The NMR spectra showed the disappearance of the hydride peak from $\mathbf{7}$ and the appearance of a $\mathrm{H}_{2}(\mathrm{~g})$ peak while the 1:6:6 peak family remained for the protons present on the ${ }^{\mathrm{Me}} \mathrm{NacNac}$ ligand still bound to the $\mathrm{Al}$ centre. Literature reports a similar avenue of decomposition for dialkylaminoalanes $\left(\mathrm{H}_{2} \mathrm{Al}\left(\mathrm{NR}_{2}\right)\right)$ after reflux in a hydrocarbon solvent, which have been shown to form a bis species $\left(\operatorname{HAl}\left(\mathrm{NR}_{2}\right)_{2}\right)$ with the evolution of hydrogen gas. ${ }^{240}$ However, while the decomposition of $\mathbf{7}$ does show the evolution of hydrogen gas and the maintenance of a 1:6:6 peak family for the MeNacNac ligand, it does not appear form $\mathrm{HAl}\left({ }^{\mathrm{MeNacNac}}\right)_{2}$ or $\mathrm{Al}\left({ }^{\mathrm{MeNacNac}}\right)\left({ }^{\mathrm{r}, \mathrm{Me}} \mathrm{NacNac}\right)(\mathbf{8})$.

Compound 8 demonstrates the first reported existence of the "reduced" MeNacNac ligand in $\mathrm{Al}\left({ }^{\mathrm{MeNacNac}}\right)\left(\mathrm{NCH}_{3}\right) \mathrm{CH}\left(\mathrm{CH}_{3}\right) \mathrm{CHC}\left(\mathrm{CH}_{3}\right)\left(\mathrm{NCH}_{3}\right)$, shown in Figure 5.23. A similar structure has been obtained in the literature for a ${ }^{\mathrm{Ph}} \mathrm{NacNac}$ aluminum species (which contains one "normal" ${ }^{P h N a c N a c ~ l i g a n d ~ a n d ~ o n e ~ r e d u c e d ~}$ 
${ }^{\mathrm{Ph}} \mathrm{NacNac}$ ligand), ${ }^{241}$ and exists likely due to an intramolecular hydroalumination reaction that occurs during precursor synthesis. The TGA of 8 (Figure 5.21c) demonstrates that the compound is thermally stable, but is less volatile than compound 6 which can be observed by its higher onset of volatilization. Its calculated 1 Torr temperature is $131{ }^{\circ} \mathrm{C}$.

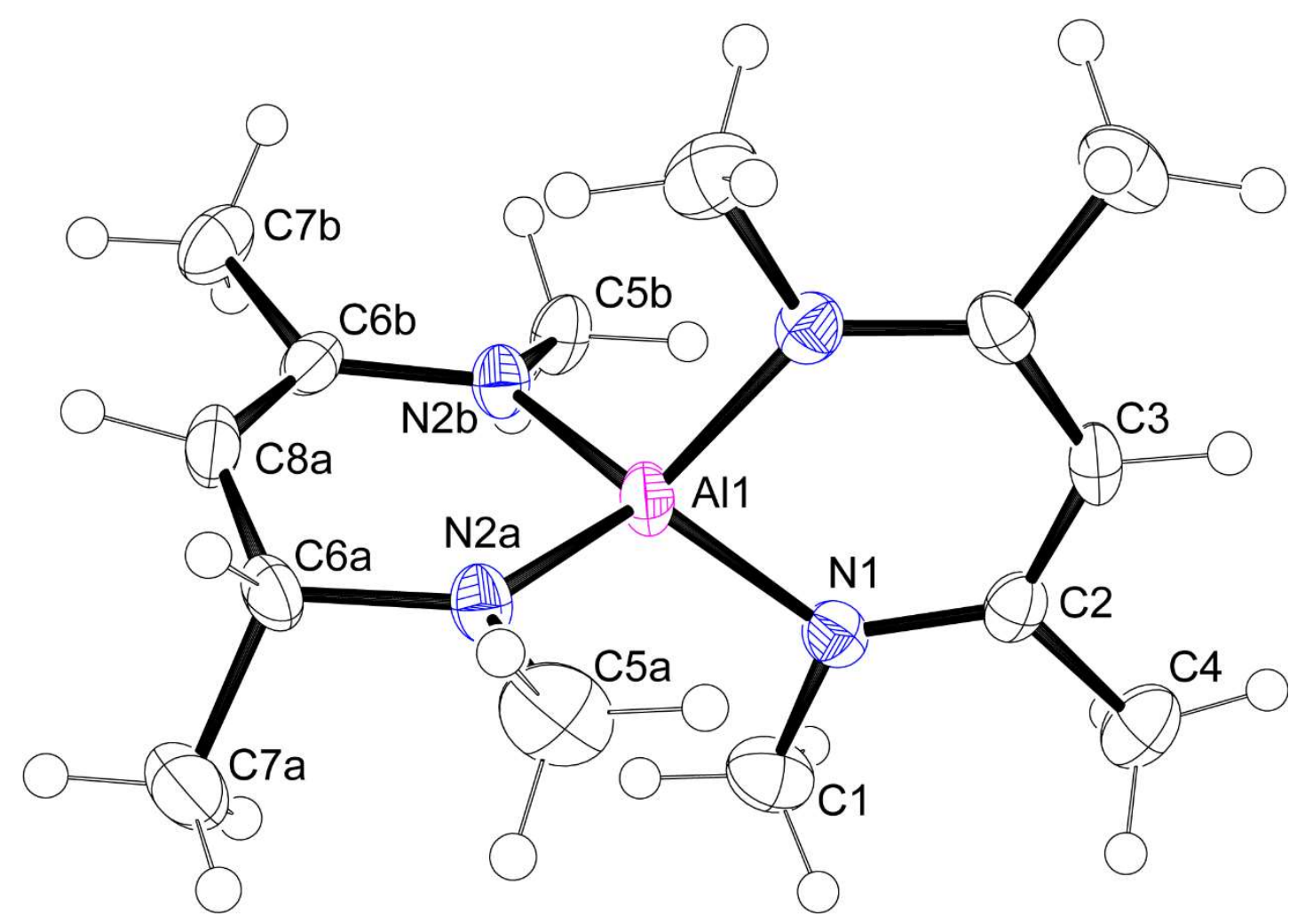

Figure 5.23: Crystal structure of compound $\mathbf{8}$ collected by single-crystal X-ray crystallography. 


\subsubsection{Summary and Conclusions}

Both novel compounds $\mathbf{6}$ and $\mathbf{8}$ have been synthesized and evaluated as potential precursors for aluminum nitride or aluminum oxide ALD. Compound 6 has an increased volatility compared to compound $\mathbf{8}$ with 1 Torr temperatures of 74 and $131{ }^{\circ} \mathrm{C}$, respectively, though both are thermally stable and do not appear to decompose by TGA. They also both maintain metal-nitrogen bonds that would ideally reduce carbon contamination when depositing AlN films. Due to the oxobridged structure that was observed upon the exposure of 6 to atmosphere, future work on these compounds could include their reaction with and activation of hydrazines to produce new potential ligands. The novel reduced ${ }^{\mathrm{MeNacNac}}$ ligand in compound $\mathbf{8}$ could also be isolated and employed with other metal centres to produce interesting potential ALD precursors. While compound $\mathbf{2}$ was hypothesized to be useful for AlN ALD due to its Al-N bonds and hydride ligands, its TGA and NMR boilup demonstrated that it was thermally unstable and therefore unsuitable for use in this process. 


\subsubsection{Supporting Information}

Table 5.12: Crystal data and structure refinement for compound $\mathbf{8}$.

\begin{tabular}{|c|c|c|}
\hline Identification code & 2018_120_26_sb_sb_b86_0ma_a & \\
\hline Empirical formula & $\mathrm{C} 14 \overline{\mathrm{H}} 27 \overline{\mathrm{Al}} \mathrm{N} 4$ & \\
\hline Formula weight & 278.37 & \\
\hline Temperature & $125.0 \mathrm{~K}$ & \\
\hline Wavelength & $0.71073 \AA$ & \\
\hline Crystal system & Monoclinic & \\
\hline Space group & I $12 / \mathrm{a} 1$ & \\
\hline \multirow[t]{3}{*}{ Unit cell dimensions } & $\mathrm{a}=12.160(2) \AA$ & $\alpha=90^{\circ}$. \\
\hline & $\mathrm{b}=10.550(2) \AA$ & $\beta=108.407(2)^{\circ}$. \\
\hline & $\mathrm{c}=13.330(3) \AA$ & $\gamma=90^{\circ}$ \\
\hline Volume & $1622.6(5) \AA^{3}$ & \\
\hline $\mathrm{Z}$ & 4 & \\
\hline Density (calculated) & $1.140 \mathrm{Mg} / \mathrm{m}^{3}$ & \\
\hline Absorption coefficient & $0.120 \mathrm{~mm}^{-1}$ & \\
\hline $\mathrm{F}(000)$ & 608 & \\
\hline Crystal size & $0.35 \times 0.3 \times 0.21 \mathrm{~mm}^{3}$ & \\
\hline Theta range for data collection & 2.514 to $29.205^{\circ}$. & \\
\hline Index ranges & $\begin{array}{l}-16<=\mathrm{h}<=16,-14<=\mathrm{k}<=14,- \\
18<=\mathrm{l}<=18\end{array}$ & \\
\hline Reflections collected & 9963 & \\
\hline Independent reflections & $2088[\mathrm{R}(\mathrm{int})=0.0399]$ & \\
\hline Completeness to theta $=25.242^{\circ}$ & $100.0 \%$ & \\
\hline Absorption correction & Semi-empirical from equivalents & \\
\hline Max. and min. transmission & 0.7458 and 0.6838 & \\
\hline Refinement method & Full-matrix least-squares on $\mathrm{F}^{2}$ & \\
\hline Data / restraints / parameters & $2088 / 1 / 131$ & \\
\hline Goodness-of-fit on $\mathrm{F}^{2}$ & 1.060 & \\
\hline Final $R$ indices $[\mathrm{I}>2 \operatorname{sigma}(\mathrm{I})]$ & $\mathrm{R} 1=0.0376, \mathrm{wR} 2=0.0950$ & \\
\hline $\mathrm{R}$ indices (all data) & $\mathrm{R} 1=0.0525, \mathrm{wR} 2=0.1035$ & \\
\hline Extinction coefficient & $\mathrm{n} / \mathrm{a}$ & \\
\hline Largest diff. peak and hole & 0.237 and -0.210 e. $\AA^{-3}$ & \\
\hline
\end{tabular}


Table 5.13: Atomic coordinates (x 104) and equivalent isotropic displacement parameters $\left(\AA^{2} \times 10^{3}\right)$ for 8 . $\mathrm{U}(\mathrm{eq})$ is defined as one third of the trace of the orthogonalized Uij tensor.

\begin{tabular}{lllll}
\hline & \multicolumn{1}{c}{$\mathrm{x}$} & \multicolumn{1}{c}{$\mathrm{y}$} & \multicolumn{1}{c}{$\mathrm{z}$} & $\mathrm{U}(\mathrm{eq})$ \\
\hline $\mathrm{Al}(1)$ & 2500 & $7176(1)$ & 5000 & $24(1)$ \\
$\mathrm{N}(1)$ & $3650(1)$ & $8365(1)$ & $5658(1)$ & $25(1)$ \\
$\mathrm{C}(2)$ & $3527(1)$ & $9621(1)$ & $5604(1)$ & $25(1)$ \\
$\mathrm{C}(3)$ & 2500 & $10210(2)$ & 5000 & $27(1)$ \\
$\mathrm{C}(4)$ & $4520(1)$ & $10457(1)$ & $6203(1)$ & $38(1)$ \\
$\mathrm{C}(1)$ & $4770(1)$ & $7821(1)$ & $6281(1)$ & $38(1)$ \\
$\mathrm{C}(6 \mathrm{~B})$ & $2484(2)$ & $4887(2)$ & $3939(2)$ & $24(1)$ \\
$\mathrm{C}(6 \mathrm{~A})$ & $3196(2)$ & $4741(2)$ & $4407(2)$ & $26(1)$ \\
$\mathrm{N}(2 \mathrm{~B})$ & $2824(5)$ & $6167(10)$ & $4053(7)$ & $24(1)$ \\
$\mathrm{N}(2 \mathrm{~A})$ & $3139(5)$ & $6105(10)$ & $4258(7)$ & $24(1)$ \\
$\mathrm{C}(5 \mathrm{~A})$ & $3637(14)$ & $6616(13)$ & $3449(11)$ & $48(3)$ \\
$\mathrm{C}(5 \mathrm{~B})$ & $3613(10)$ & $6534(10)$ & $3504(9)$ & $28(2)$ \\
$\mathrm{C}(7 \mathrm{~A})$ & $2364(3)$ & $4040(3)$ & $3473(3)$ & $43(1)$ \\
$\mathrm{C}(7 \mathrm{~B})$ & $2631(3)$ & $4169(3)$ & $3013(3)$ & $36(1)$ \\
$\mathrm{C}(8 \mathrm{~A})$ & $1999(2)$ & $4297(2)$ & $4588(2)$ & $30(1)$ \\
\hline
\end{tabular}

Table 5.14: Bond lengths for 8 .

\begin{tabular}{llll}
\hline $\mathrm{Al}(1)-\mathrm{N}(1) \# 1$ & $1.8780(11)$ & $\mathrm{C}(4)-\mathrm{H}(4 \mathrm{~A})$ & 0.9800 \\
$\mathrm{Al}(1)-\mathrm{N}(1)$ & $1.8779(11)$ & $\mathrm{C}(4)-\mathrm{H}(4 \mathrm{~B})$ & 0.9800 \\
$\mathrm{Al}(1)-\mathrm{N}(2 \mathrm{~B})$ & $1.787(10)$ & $\mathrm{C}(4)-\mathrm{H}(4 \mathrm{C})$ & 0.9800 \\
$\mathrm{Al}(1)-\mathrm{N}(2 \mathrm{~B}) \# 1$ & $1.787(10)$ & $\mathrm{C}(1)-\mathrm{H}(1 \mathrm{~A})$ & 0.9800 \\
$\mathrm{Al}(1)-\mathrm{N}(2 \mathrm{~A})$ & $1.829(10)$ & $\mathrm{C}(1)-\mathrm{H}(1 \mathrm{~B})$ & 0.9800 \\
$\mathrm{Al}(1)-\mathrm{N}(2 \mathrm{~A}) \# 1$ & $1.829(10)$ & $\mathrm{C}(1)-\mathrm{H}(1 \mathrm{C})$ & 0.9800 \\
$\mathrm{~N}(1)-\mathrm{C}(2)$ & $1.3323(16)$ & $\mathrm{C}(6 \mathrm{~B})-\mathrm{N}(2 \mathrm{~B})$ & $1.407(11)$ \\
$\mathrm{N}(1)-\mathrm{C}(1)$ & $1.4695(16)$ & $\mathrm{C}(6 \mathrm{~B})-\mathrm{C}(7 \mathrm{~B})$ & $1.506(4)$ \\
$\mathrm{C}(2)-\mathrm{C}(3)$ & $1.3997(15)$ & $\mathrm{C}(6 \mathrm{~B})-\mathrm{C}(8 \mathrm{~A})$ & $1.344(4)$ \\
$\mathrm{C}(2)-\mathrm{C}(4)$ & $1.5055(17)$ & $\mathrm{C}(6 \mathrm{~A})-\mathrm{H}(6 \mathrm{~A})$ & 1.0000 \\
$\mathrm{C}(3)-\mathrm{C}(2) \# 1$ & $1.3997(15)$ & & \\
$\mathrm{C}(3)-\mathrm{H}(3)$ & 0.9500 & &
\end{tabular}




\begin{tabular}{|c|c|c|c|}
\hline $\mathrm{C}(6 \mathrm{~A})-\mathrm{N}(2 \mathrm{~A})$ & $1.451(11)$ & $\mathrm{N}(2 \mathrm{~A}) \# 1-\mathrm{Al}(1)-\mathrm{N}(1) \# 1$ & 106.6(3) \\
\hline $\mathrm{C}(6 \mathrm{~A})-\mathrm{C}(7 \mathrm{~A})$ & $1.526(4)$ & $\mathrm{N}(2 \mathrm{~A}) \# 1-\mathrm{Al}(1)-\mathrm{N}(1)$ & $122.8(2)$ \\
\hline $\mathrm{C}(6 \mathrm{~A})-\mathrm{C}(8 \mathrm{~A}) \# 1$ & $1.507(4)$ & $\mathrm{N}(2 \mathrm{~A})-\mathrm{Al}(1)-\mathrm{N}(1) \# 1$ & $122.8(2)$ \\
\hline $\mathrm{N}(2 \mathrm{~B})-\mathrm{C}(5 \mathrm{~B})$ & $1.431(17)$ & $\mathrm{N}(2 \mathrm{~A})-\mathrm{Al}(1)-\mathrm{N}(2 \mathrm{~A}) \# 1$ & $103.7(7)$ \\
\hline $\mathrm{N}(2 \mathrm{~A})-\mathrm{C}(5 \mathrm{~A})$ & $1.495(19)$ & $\mathrm{C}(2)-\mathrm{N}(1)-\mathrm{Al}(1)$ & $125.80(8)$ \\
\hline $\mathrm{C}(5 \mathrm{~A})-\mathrm{H}(5 \mathrm{AA})$ & 0.9800 & $\mathrm{C}(2)-\mathrm{N}(1)-\mathrm{C}(1)$ & $119.19(11)$ \\
\hline $\mathrm{C}(5 \mathrm{~A})-\mathrm{H}(5 \mathrm{AB})$ & 0.9800 & $\mathrm{C}(1)-\mathrm{N}(1)-\mathrm{Al}(1)$ & $115.02(9)$ \\
\hline $\mathrm{C}(5 \mathrm{~A})-\mathrm{H}(5 \mathrm{AC})$ & 0.9800 & $\mathrm{~N}(1)-\mathrm{C}(2)-\mathrm{C}(3)$ & $122.48(12)$ \\
\hline $\mathrm{C}(5 \mathrm{~B})-\mathrm{H}(5 \mathrm{BA})$ & 0.9800 & $\mathrm{~N}(1)-\mathrm{C}(2)-\mathrm{C}(4)$ & $119.77(11)$ \\
\hline $\mathrm{C}(5 \mathrm{~B})-\mathrm{H}(5 \mathrm{BB})$ & 0.9800 & $\mathrm{C}(3)-\mathrm{C}(2)-\mathrm{C}(4)$ & $117.75(12)$ \\
\hline $\mathrm{C}(5 \mathrm{~B})-\mathrm{H}(5 \mathrm{BC})$ & 0.9800 & $\mathrm{C}(2) \# 1-\mathrm{C}(3)-\mathrm{C}(2)$ & $127.30(16)$ \\
\hline $\mathrm{C}(7 \mathrm{~A})-\mathrm{H}(7 \mathrm{AA})$ & 0.9800 & $\mathrm{C}(2)-\mathrm{C}(3)-\mathrm{H}(3)$ & 116.4 \\
\hline $\mathrm{C}(7 \mathrm{~A})-\mathrm{H}(7 \mathrm{AB})$ & 0.9800 & $\mathrm{C}(2) \# 1-\mathrm{C}(3)-\mathrm{H}(3)$ & 116.4 \\
\hline $\mathrm{C}(7 \mathrm{~A})-\mathrm{H}(7 \mathrm{AC})$ & 0.9800 & $\mathrm{C}(2)-\mathrm{C}(4)-\mathrm{H}(4 \mathrm{~A})$ & 109.5 \\
\hline $\mathrm{C}(7 \mathrm{~B})-\mathrm{H}(7 \mathrm{BA})$ & 0.9800 & $\mathrm{C}(2)-\mathrm{C}(4)-\mathrm{H}(4 \mathrm{~B})$ & 109.5 \\
\hline $\mathrm{C}(7 \mathrm{~B})-\mathrm{H}(7 \mathrm{BB})$ & 0.9800 & $\mathrm{C}(2)-\mathrm{C}(4)-\mathrm{H}(4 \mathrm{C})$ & 109.5 \\
\hline $\mathrm{C}(7 \mathrm{~B})-\mathrm{H}(7 \mathrm{BC})$ & 0.9800 & $\mathrm{H}(4 \mathrm{~A})-\mathrm{C}(4)-\mathrm{H}(4 \mathrm{~B})$ & 109.5 \\
\hline $\mathrm{C}(8 \mathrm{~A})-\mathrm{C}(6 \mathrm{~A}) \# 1$ & $1.507(4)$ & $\mathrm{H}(4 \mathrm{~A})-\mathrm{C}(4)-\mathrm{H}(4 \mathrm{C})$ & 109.5 \\
\hline \multirow[t]{2}{*}{$\mathrm{C}(8 \mathrm{~A})-\mathrm{H}(8 \mathrm{~A})$} & $0.95000(19)$ & $\mathrm{H}(4 \mathrm{~B})-\mathrm{C}(4)-\mathrm{H}(4 \mathrm{C})$ & 109.5 \\
\hline & & $\mathrm{N}(1)-\mathrm{C}(1)-\mathrm{H}(1 \mathrm{~A})$ & 109.5 \\
\hline $\mathrm{N}(1)-\mathrm{Al}(1)-\mathrm{N}(1) \# 1$ & $96.11(7)$ & $\mathrm{N}(1)-\mathrm{C}(1)-\mathrm{H}(1 \mathrm{~B})$ & 109.5 \\
\hline $\mathrm{N}(2 \mathrm{~B})-\mathrm{Al}(1)-\mathrm{N}(1)$ & $115.5(3)$ & $\mathrm{N}(1)-\mathrm{C}(1)-\mathrm{H}(1 \mathrm{C})$ & 109.5 \\
\hline $\mathrm{N}(2 \mathrm{~B}) \# 1-\mathrm{Al}(1)-\mathrm{N}(1) \# 1$ & $115.5(3)$ & $\mathrm{H}(1 \mathrm{~A})-\mathrm{C}(1)-\mathrm{H}(1 \mathrm{~B})$ & 109.5 \\
\hline $\mathrm{N}(2 \mathrm{~B}) \# 1-\mathrm{Al}(1)-\mathrm{N}(1)$ & $111.4(2)$ & $\mathrm{H}(1 \mathrm{~A})-\mathrm{C}(1)-\mathrm{H}(1 \mathrm{C})$ & 109.5 \\
\hline $\mathrm{N}(2 \mathrm{~B})-\mathrm{Al}(1)-\mathrm{N}(1) \# 1$ & $111.4(2)$ & $\mathrm{H}(1 \mathrm{~B})-\mathrm{C}(1)-\mathrm{H}(1 \mathrm{C})$ & 109.5 \\
\hline $\mathrm{N}(2 \mathrm{~B}) \# 1-\mathrm{Al}(1)-\mathrm{N}(2 \mathrm{~B})$ & $107.0(7)$ & $\mathrm{N}(2 \mathrm{~B})-\mathrm{C}(6 \mathrm{~B})-\mathrm{C}(7 \mathrm{~B})$ & $117.7(5)$ \\
\hline $\mathrm{N}(2 \mathrm{~A})-\mathrm{Al}(1)-\mathrm{N}(1)$ & 106.6(3) & $\mathrm{N}(2 \mathrm{~B})-\mathrm{C}(6 \mathrm{~B})-\mathrm{C}(8 \mathrm{~A}) \# 1$ & $101.9(4)$ \\
\hline
\end{tabular}




\begin{tabular}{|c|c|c|c|}
\hline $\mathrm{C}(7 \mathrm{~B})-\mathrm{C}(6 \mathrm{~B})-\mathrm{C}(8 \mathrm{~A}) \# 1$ & $125.2(2)$ & $\mathrm{C}(6 \mathrm{~A})-\mathrm{C}(7 \mathrm{~A})-\mathrm{H}(7 \mathrm{AA})$ & 109.5 \\
\hline $\mathrm{C}(8 \mathrm{~A})-\mathrm{C}(6 \mathrm{~B})-\mathrm{N}(2 \mathrm{~B})$ & $123.3(5)$ & $\mathrm{C}(6 \mathrm{~A})-\mathrm{C}(7 \mathrm{~A})-\mathrm{H}(7 \mathrm{AB})$ & 109.5 \\
\hline$C(8 A)-C(6 B)-C(7 B)$ & $119.0(2)$ & $\mathrm{C}(6 \mathrm{~A})-\mathrm{C}(7 \mathrm{~A})-\mathrm{H}(7 \mathrm{AC})$ & 109.5 \\
\hline $\mathrm{N}(2 \mathrm{~A})-\mathrm{C}(6 \mathrm{~A})-\mathrm{H}(6 \mathrm{~A})$ & 106.9 & $\mathrm{H}(7 \mathrm{AA})-\mathrm{C}(7 \mathrm{~A})-\mathrm{H}(7 \mathrm{AB})$ & 109.5 \\
\hline $\mathrm{N}(2 \mathrm{~A})-\mathrm{C}(6 \mathrm{~A})-\mathrm{C}(7 \mathrm{~A})$ & $112.2(3)$ & $\mathrm{H}(7 \mathrm{AA})-\mathrm{C}(7 \mathrm{~A})-\mathrm{H}(7 \mathrm{AC})$ & 109.5 \\
\hline $\mathrm{N}(2 \mathrm{~A})-\mathrm{C}(6 \mathrm{~A})-\mathrm{C}(8 \mathrm{~A}) \# 1$ & $114.6(4)$ & $\mathrm{H}(7 \mathrm{AB})-\mathrm{C}(7 \mathrm{~A})-\mathrm{H}(7 \mathrm{AC})$ & 109.5 \\
\hline $\mathrm{C}(7 \mathrm{~A})-\mathrm{C}(6 \mathrm{~A})-\mathrm{H}(6 \mathrm{~A})$ & 106.9 & $\mathrm{C}(6 \mathrm{~B})-\mathrm{C}(7 \mathrm{~B})-\mathrm{H}(7 \mathrm{BA})$ & 109.5 \\
\hline $\mathrm{C}(8 \mathrm{~A}) \# 1-\mathrm{C}(6 \mathrm{~A})-\mathrm{H}(6 \mathrm{~A})$ & 106.9 & $\mathrm{C}(6 \mathrm{~B})-\mathrm{C}(7 \mathrm{~B})-\mathrm{H}(7 \mathrm{BB})$ & 109.5 \\
\hline $\mathrm{C}(8 \mathrm{~A}) \# 1-\mathrm{C}(6 \mathrm{~A})-\mathrm{C}(7 \mathrm{~A})$ & $109.0(2)$ & $\mathrm{C}(6 \mathrm{~B})-\mathrm{C}(7 \mathrm{~B})-\mathrm{H}(7 \mathrm{BC})$ & 109.5 \\
\hline $\mathrm{C}(6 \mathrm{~B})-\mathrm{N}(2 \mathrm{~B})-\mathrm{Al}(1)$ & $121.7(7)$ & $\mathrm{H}(7 \mathrm{BA})-\mathrm{C}(7 \mathrm{~B})-\mathrm{H}(7 \mathrm{BB})$ & 109.5 \\
\hline $\mathrm{C}(6 \mathrm{~B})-\mathrm{N}(2 \mathrm{~B})-\mathrm{C}(5 \mathrm{~B})$ & $115.2(8)$ & $\mathrm{H}(7 \mathrm{BA})-\mathrm{C}(7 \mathrm{~B})-\mathrm{H}(7 \mathrm{BC})$ & 109.5 \\
\hline $\mathrm{C}(5 \mathrm{~B})-\mathrm{N}(2 \mathrm{~B})-\mathrm{Al}(1)$ & $122.2(8)$ & $\mathrm{H}(7 \mathrm{BB})-\mathrm{C}(7 \mathrm{~B})-\mathrm{H}(7 \mathrm{BC})$ & 109.5 \\
\hline $\mathrm{C}(6 \mathrm{~A})-\mathrm{N}(2 \mathrm{~A})-\mathrm{Al}(1)$ & $123.3(7)$ & $\mathrm{C}(6 \mathrm{~B})-\mathrm{C}(8 \mathrm{~A})-\mathrm{H}(8 \mathrm{~A})$ & $115.0(11)$ \\
\hline$C(6 A)-N(2 A)-C(5 A)$ & $116.3(8)$ & $\mathrm{C}(6 \mathrm{~A}) \# 1-\mathrm{C}(8 \mathrm{~A})-\mathrm{H}(8 \mathrm{~A})$ & $114.3(11)$ \\
\hline $\mathrm{C}(5 \mathrm{~A})-\mathrm{N}(2 \mathrm{~A})-\mathrm{Al}(1)$ & $120.4(8)$ & & \\
\hline $\mathrm{N}(2 \mathrm{~A})-\mathrm{C}(5 \mathrm{~A})-\mathrm{H}(5 \mathrm{AA})$ & 109.5 & & \\
\hline $\mathrm{N}(2 \mathrm{~A})-\mathrm{C}(5 \mathrm{~A})-\mathrm{H}(5 \mathrm{AB})$ & 109.5 & & \\
\hline $\mathrm{N}(2 \mathrm{~A})-\mathrm{C}(5 \mathrm{~A})-\mathrm{H}(5 \mathrm{AC})$ & 109.5 & & \\
\hline $\mathrm{H}(5 \mathrm{AA})-\mathrm{C}(5 \mathrm{~A})-\mathrm{H}(5 \mathrm{AB})$ & 109.5 & & \\
\hline $\mathrm{H}(5 \mathrm{AA})-\mathrm{C}(5 \mathrm{~A})-\mathrm{H}(5 \mathrm{AC})$ & 109.5 & & \\
\hline $\mathrm{H}(5 \mathrm{AB})-\mathrm{C}(5 \mathrm{~A})-\mathrm{H}(5 \mathrm{AC})$ & 109.5 & & \\
\hline $\mathrm{N}(2 \mathrm{~B})-\mathrm{C}(5 \mathrm{~B})-\mathrm{H}(5 \mathrm{BA})$ & 109.5 & & \\
\hline $\mathrm{N}(2 \mathrm{~B})-\mathrm{C}(5 \mathrm{~B})-\mathrm{H}(5 \mathrm{BB})$ & 109.5 & & \\
\hline $\mathrm{N}(2 \mathrm{~B})-\mathrm{C}(5 \mathrm{~B})-\mathrm{H}(5 \mathrm{BC})$ & 109.5 & & \\
\hline $\mathrm{H}(5 \mathrm{BA})-\mathrm{C}(5 \mathrm{~B})-\mathrm{H}(5 \mathrm{BB})$ & 109.5 & & \\
\hline $\mathrm{H}(5 \mathrm{BA})-\mathrm{C}(5 \mathrm{~B})-\mathrm{H}(5 \mathrm{BC})$ & 109.5 & & \\
\hline $\mathrm{H}(5 \mathrm{BB})-\mathrm{C}(5 \mathrm{~B})-\mathrm{H}(5 \mathrm{BC})$ & 109.5 & & \\
\hline
\end{tabular}


Table 5.15: Anisotropic displacement parameters $\left(\AA^{2} \mathrm{x} 10^{3}\right)$ for 8 . The anisotropic displacement factor exponent takes the form: $-2 \pi^{2}\left[\mathrm{~h}^{2} \mathrm{a}^{* 2} \mathrm{U}^{11}+\ldots+2 \mathrm{~h} \mathrm{k} \mathrm{a}^{*}\right.$ $\left.b^{*} \mathrm{U}^{12}\right]$.

\begin{tabular}{lllllll}
\hline & $\mathrm{U}^{11}$ & $\mathrm{U}^{22}$ & $\mathrm{U}^{33}$ & $\mathrm{U}^{23}$ & $\mathrm{U}^{13}$ & $\mathrm{U}^{12}$ \\
\hline $\mathrm{Al}(1)$ & $38(1)$ & $16(1)$ & $25(1)$ & 0 & $17(1)$ & 0 \\
$\mathrm{~N}(1)$ & $27(1)$ & $24(1)$ & $25(1)$ & $1(1)$ & $10(1)$ & $4(1)$ \\
$\mathrm{C}(2)$ & $30(1)$ & $23(1)$ & $24(1)$ & $-4(1)$ & $14(1)$ & $-4(1)$ \\
$\mathrm{C}(3)$ & $35(1)$ & $15(1)$ & $35(1)$ & 0 & $15(1)$ & 0 \\
$\mathrm{C}(4)$ & $37(1)$ & $38(1)$ & $40(1)$ & $-9(1)$ & $12(1)$ & $-11(1)$ \\
$\mathrm{C}(1)$ & $32(1)$ & $45(1)$ & $37(1)$ & $5(1)$ & $9(1)$ & $11(1)$ \\
$\mathrm{C}(6 \mathrm{~B})$ & $20(1)$ & $22(1)$ & $26(1)$ & $-6(1)$ & $2(1)$ & $1(1)$ \\
$\mathrm{C}(6 \mathrm{~A})$ & $27(1)$ & $20(1)$ & $31(1)$ & $-7(1)$ & $9(1)$ & $3(1)$ \\
$\mathrm{N}(2 \mathrm{~B})$ & $26(3)$ & $19(1)$ & $30(3)$ & $-1(2)$ & $14(2)$ & $1(2)$ \\
$\mathrm{N}(2 \mathrm{~A})$ & $26(3)$ & $19(1)$ & $30(3)$ & $-1(2)$ & $14(2)$ & $1(2)$ \\
$\mathrm{C}(5 \mathrm{~A})$ & $60(6)$ & $56(6)$ & $40(5)$ & $-3(3)$ & $34(4)$ & $-19(4)$ \\
$\mathrm{C}(5 \mathrm{~B})$ & $34(4)$ & $24(3)$ & $33(4)$ & $-9(3)$ & $21(3)$ & $-2(2)$ \\
$\mathrm{C}(7 \mathrm{~A})$ & $55(2)$ & $33(2)$ & $40(2)$ & $-11(2)$ & $14(2)$ & $-7(1)$ \\
$\mathrm{C}(7 \mathrm{~B})$ & $35(2)$ & $33(2)$ & $38(2)$ & $-15(1)$ & $9(1)$ & $3(1)$ \\
$\mathrm{C}(8 \mathrm{~A})$ & $30(1)$ & $16(1)$ & $40(2)$ & $-2(1)$ & $7(1)$ & $-3(1)$ \\
\hline
\end{tabular}


Table 5.16: Hydrogen coordinates (x 104) and isotropic displacement parameters $\left(\AA^{2} \times 10^{3}\right)$ for 8.

\begin{tabular}{|c|c|c|c|c|}
\hline & $\mathrm{x}$ & $\mathrm{y}$ & $\mathrm{z}$ & $\mathrm{U}(\mathrm{eq})$ \\
\hline $\mathrm{H}(3)$ & 2500 & 11110 & 5000 & 33 \\
\hline $\mathrm{H}(4 \mathrm{~A})$ & 4733 & 10269 & 6960 & 57 \\
\hline $\mathrm{H}(4 \mathrm{~B})$ & 4288 & 11348 & 6082 & 57 \\
\hline $\mathrm{H}(4 \mathrm{C})$ & 5187 & 10300 & 5958 & 57 \\
\hline $\mathrm{H}(1 \mathrm{~A})$ & 4963 & 8101 & 7017 & 57 \\
\hline $\mathrm{H}(1 \mathrm{~B})$ & 5374 & 8104 & 5989 & 57 \\
\hline $\mathrm{H}(1 \mathrm{C})$ & 4720 & 6893 & 6251 & 57 \\
\hline $\mathrm{H}(6 \mathrm{~A})$ & 3998 & 4474 & 4447 & 32 \\
\hline $\mathrm{H}(5 \mathrm{AA})$ & 3204 & 6276 & 2752 & 72 \\
\hline $\mathrm{H}(5 \mathrm{AB})$ & 4452 & 6363 & 3630 & 72 \\
\hline $\mathrm{H}(5 \mathrm{AC})$ & 3582 & 7543 & 3435 & 72 \\
\hline $\mathrm{H}(5 \mathrm{BA})$ & 3750 & 7450 & 3580 & 42 \\
\hline $\mathrm{H}(5 \mathrm{BB})$ & 3281 & 6321 & 2753 & 42 \\
\hline $\mathrm{H}(5 \mathrm{BC})$ & 4349 & 6083 & 3804 & 42 \\
\hline $\mathrm{H}(7 \mathrm{AA})$ & 2512 & 4292 & 2819 & 64 \\
\hline $\mathrm{H}(7 \mathrm{AB})$ & 1564 & 4253 & 3422 & 64 \\
\hline $\mathrm{H}(7 \mathrm{AC})$ & 2481 & 3124 & 3576 & 64 \\
\hline $\mathrm{H}(7 \mathrm{BA})$ & 2327 & 3308 & 3004 & 54 \\
\hline $\mathrm{H}(7 \mathrm{BB})$ & 3456 & 4129 & 3076 & 54 \\
\hline $\mathrm{H}(7 \mathrm{BC})$ & 2206 & 4604 & 2354 & 54 \\
\hline $\mathrm{H}(8 \mathrm{~A})$ & $1731(10)$ & $3461(3)$ & $4380(11)$ & 45 \\
\hline
\end{tabular}


Table 5.17: Crystal data and structure refinement for compound 6 .

\begin{tabular}{|c|c|c|}
\hline Identification code & 2018_04_21_sbsb04_0m_a & \\
\hline Empirical formula & C18 H38 Al2 N6 O & \\
\hline Formula weight & 408.50 & \\
\hline Temperature & $125.0 \mathrm{~K}$ & \\
\hline Wavelength & $0.71073 \AA$ & \\
\hline Crystal system & Monoclinic & \\
\hline Space group & P 1 21/n 1 & \\
\hline \multirow[t]{3}{*}{ Unit cell dimensions } & $\mathrm{a}=8.377(2) \AA$ & $\alpha=90^{\circ}$ \\
\hline & $\mathrm{b}=23.610(6) \AA$ & $\beta=90.320(3)^{\circ}$. \\
\hline & $\mathrm{c}=11.868(3) \AA$ & $\gamma=90^{\circ}$ \\
\hline Volume & $2347.3(10) \AA^{3}$ & \\
\hline $\mathrm{Z}$ & 4 & \\
\hline Density (calculated) & $1.156 \mathrm{Mg} / \mathrm{m}^{3}$ & \\
\hline Absorption coefficient & $0.143 \mathrm{~mm}^{-1}$ & \\
\hline $\mathrm{F}(000)$ & 888 & \\
\hline Crystal size & $0.11 \times 0.08 \times 0.04 \mathrm{~mm}^{3}$ & \\
\hline Theta range for data collection & 1.725 to $25.500^{\circ}$. & \\
\hline Index ranges & $\begin{array}{l}-10<=\mathrm{h}<=10,-28<=\mathrm{k}<=28,- \\
14<=\mathrm{l}<=14\end{array}$ & \\
\hline Reflections collected & 23706 & \\
\hline Independent reflections & $4358[\mathrm{R}(\mathrm{int})=0.0832]$ & \\
\hline Completeness to theta $=25.242^{\circ}$ & $99.7 \%$ & \\
\hline Absorption correction & Semi-empirical from equivalents & \\
\hline Max. and min. transmission & 0.7457 and 0.6046 & \\
\hline Refinement method & Full-matrix least-squares on $\mathrm{F}^{2}$ & \\
\hline Data / restraints / parameters & $4358 / 0 / 256$ & \\
\hline Goodness-of-fit on $\mathrm{F}^{2}$ & 1.093 & \\
\hline Final $R$ indices $[\mathrm{I}>2 \operatorname{sigma}(\mathrm{I})]$ & $\mathrm{R} 1=0.0672, \mathrm{wR} 2=0.1635$ & \\
\hline $\mathrm{R}$ indices (all data) & $\mathrm{R} 1=0.1043, \mathrm{wR} 2=0.1801$ & \\
\hline Extinction coefficient & $\mathrm{n} / \mathrm{a}$ & \\
\hline Largest diff. peak and hole & 0.292 and -0.297 e. $\AA^{-3}$ & \\
\hline
\end{tabular}


Table 5.18: Atomic coordinates (x 104) and equivalent isotropic displacement parameters $\left(\AA^{2} \times 10^{3}\right)$ for 6 . $\mathrm{U}(\mathrm{eq})$ is defined as one third of the trace of the orthogonalized Uij tensor.

\begin{tabular}{|c|c|c|c|c|}
\hline & $\mathrm{x}$ & $\mathrm{y}$ & $\mathrm{z}$ & $\mathrm{U}(\mathrm{eq})$ \\
\hline $\operatorname{Al}(2)$ & $5106(2)$ & $6415(1)$ & $4339(1)$ & $26(1)$ \\
\hline $\operatorname{Al}(1)$ & $5802(2)$ & $6295(1)$ & $1544(1)$ & $26(1)$ \\
\hline $\mathrm{O}(1)$ & $5385(4)$ & $6373(1)$ & $2932(2)$ & $34(1)$ \\
\hline $\mathrm{N}(3)$ & $5533(4)$ & $6970(1)$ & $713(3)$ & $28(1)$ \\
\hline $\mathrm{N}(2)$ & $4219(4)$ & $5878(1)$ & $755(3)$ & $26(1)$ \\
\hline $\mathrm{N}(6)$ & $6676(4)$ & $6062(1)$ & $5249(3)$ & $30(1)$ \\
\hline $\mathrm{N}(5)$ & $5480(4)$ & $7159(1)$ & $4874(3)$ & $28(1)$ \\
\hline $\mathrm{N}(4)$ & $3206(4)$ & $6146(2)$ & $4794(3)$ & $34(1)$ \\
\hline $\mathrm{N}(1)$ & $7743(4)$ & $5997(2)$ & $1259(3)$ & $34(1)$ \\
\hline $\mathrm{C}(4)$ & $3685(5)$ & $6011(2)$ & $-277(3)$ & $28(1)$ \\
\hline $\mathrm{C}(13)$ & $6223(5)$ & $7283(2)$ & $5847(4)$ & $31(1)$ \\
\hline $\mathrm{C}(16)$ & $7291(5)$ & $6297(2)$ & $6179(3)$ & $30(1)$ \\
\hline $\mathrm{C}(3)$ & $3675(5)$ & $5346(2)$ & $1278(4)$ & $33(1)$ \\
\hline $\mathrm{C}(15)$ & $7038(5)$ & $6863(2)$ & $6478(3)$ & $31(1)$ \\
\hline $\mathrm{C}(7)$ & $4816(5)$ & $6998(2)$ & $-298(4)$ & $31(1)$ \\
\hline $\mathrm{C}(6)$ & $4032(5)$ & $6534(2)$ & $-791(4)$ & $32(1)$ \\
\hline $\mathrm{C}(12)$ & $4759(6)$ & $7625(2)$ & $4225(4)$ & $35(1)$ \\
\hline $\mathrm{C}(2)$ & $8291(5)$ & $5924(2)$ & $103(4)$ & $37(1)$ \\
\hline $\mathrm{C}(9)$ & $6206(5)$ & $7494(2)$ & $1197(4)$ & $36(1)$ \\
\hline $\mathrm{C}(5)$ & $2607(5)$ & $5610(2)$ & $-902(4)$ & $37(1)$ \\
\hline $\mathrm{C}(14)$ & $6219(6)$ & $7880(2)$ & $6279(4)$ & $40(1)$ \\
\hline $\mathrm{C}(10)$ & $1851(5)$ & $6014(2)$ & $4067(4)$ & $41(1)$ \\
\hline $\mathrm{C}(8)$ & $4788(6)$ & $7553(2)$ & $-936(4)$ & $43(1)$ \\
\hline $\mathrm{C}(17)$ & $8314(6)$ & $5945(2)$ & $6971(4)$ & $44(1)$ \\
\hline $\mathrm{C}(11)$ & $2846(6)$ & $6050(2)$ & $5981(4)$ & $40(1)$ \\
\hline $\mathrm{C}(1)$ & 8913(6) & $5806(2)$ & $2069(4)$ & $43(1)$ \\
\hline $\mathrm{C}(18)$ & $7038(6)$ & $5465(2)$ & 4995(4) & $43(1)$ \\
\hline
\end{tabular}


Table 5.19: Bond lengths for 6 .

\begin{tabular}{|c|c|c|c|}
\hline $\mathrm{Al}(2)-\mathrm{O}(1)$ & $1.691(3)$ & $\mathrm{C}(12)-\mathrm{H}(12 \mathrm{~A})$ & 0.9800 \\
\hline $\mathrm{Al}(2)-\mathrm{N}(6)$ & $1.891(4)$ & $\mathrm{C}(12)-\mathrm{H}(12 \mathrm{~B})$ & 0.9800 \\
\hline $\mathrm{Al}(2)-\mathrm{N}(5)$ & $1.894(4)$ & $\mathrm{C}(12)-\mathrm{H}(12 \mathrm{C})$ & 0.9800 \\
\hline $\operatorname{Al}(2)-\mathrm{N}(4)$ & $1.800(4)$ & $\mathrm{C}(2)-\mathrm{H}(2 \mathrm{~A})$ & 0.9800 \\
\hline $\mathrm{Al}(1)-\mathrm{O}(1)$ & $1.696(3)$ & $\mathrm{C}(2)-\mathrm{H}(2 \mathrm{~B})$ & 0.9800 \\
\hline $\operatorname{Al}(1)-\mathrm{N}(3)$ & $1.887(4)$ & $\mathrm{C}(2)-\mathrm{H}(2 \mathrm{C})$ & 0.9800 \\
\hline $\mathrm{Al}(1)-\mathrm{N}(2)$ & $1.894(3)$ & $\mathrm{C}(9)-\mathrm{H}(9 \mathrm{~A})$ & 0.9800 \\
\hline $\mathrm{Al}(1)-\mathrm{N}(1)$ & $1.806(4)$ & $\mathrm{C}(9)-\mathrm{H}(9 \mathrm{~B})$ & 0.9800 \\
\hline $\mathrm{N}(3)-\mathrm{C}(7)$ & $1.340(5)$ & $\mathrm{C}(9)-\mathrm{H}(9 \mathrm{C})$ & 0.9800 \\
\hline $\mathrm{N}(3)-\mathrm{C}(9)$ & $1.475(5)$ & $\mathrm{C}(5)-\mathrm{H}(5 \mathrm{~A})$ & 0.9800 \\
\hline $\mathrm{N}(2)-\mathrm{C}(4)$ & $1.338(5)$ & $\mathrm{C}(5)-\mathrm{H}(5 \mathrm{~B})$ & 0.9800 \\
\hline $\mathrm{N}(2)-\mathrm{C}(3)$ & $1.476(5)$ & $\mathrm{C}(5)-\mathrm{H}(5 \mathrm{C})$ & 0.9800 \\
\hline $\mathrm{N}(6)-\mathrm{C}(16)$ & $1.336(5)$ & $\mathrm{C}(14)-\mathrm{H}(14 \mathrm{~A})$ & 0.9800 \\
\hline $\mathrm{N}(6)-\mathrm{C}(18)$ & $1.473(6)$ & $\mathrm{C}(14)-\mathrm{H}(14 \mathrm{~B})$ & 0.9800 \\
\hline $\mathrm{N}(5)-\mathrm{C}(13)$ & $1.341(5)$ & $\mathrm{C}(14)-\mathrm{H}(14 \mathrm{C})$ & 0.9800 \\
\hline $\mathrm{N}(5)-\mathrm{C}(12)$ & $1.470(5)$ & $\mathrm{C}(10)-\mathrm{H}(10 \mathrm{~A})$ & 0.9800 \\
\hline $\mathrm{N}(4)-\mathrm{C}(10)$ & $1.456(5)$ & $\mathrm{C}(10)-\mathrm{H}(10 \mathrm{~B})$ & 0.9800 \\
\hline $\mathrm{N}(4)-\mathrm{C}(11)$ & $1.459(6)$ & $\mathrm{C}(10)-\mathrm{H}(10 \mathrm{C})$ & 0.9800 \\
\hline $\mathrm{N}(1)-\mathrm{C}(2)$ & $1.460(5)$ & $\mathrm{C}(8)-\mathrm{H}(8 \mathrm{~A})$ & 0.9800 \\
\hline $\mathrm{N}(1)-\mathrm{C}(1)$ & $1.442(6)$ & $\mathrm{C}(8)-\mathrm{H}(8 \mathrm{~B})$ & 0.9800 \\
\hline $\mathrm{C}(4)-\mathrm{C}(6)$ & $1.408(6)$ & $\mathrm{C}(8)-\mathrm{H}(8 \mathrm{C})$ & 0.9800 \\
\hline $\mathrm{C}(4)-\mathrm{C}(5)$ & $1.501(6)$ & $\mathrm{C}(17)-\mathrm{H}(17 \mathrm{~A})$ & 0.9800 \\
\hline $\mathrm{C}(13)-\mathrm{C}(15)$ & $1.415(6)$ & $\mathrm{C}(17)-\mathrm{H}(17 \mathrm{~B})$ & 0.9800 \\
\hline $\mathrm{C}(13)-\mathrm{C}(14)$ & $1.500(6)$ & $\mathrm{C}(17)-\mathrm{H}(17 \mathrm{C})$ & 0.9800 \\
\hline $\mathrm{C}(16)-\mathrm{C}(15)$ & $1.400(6)$ & $\mathrm{C}(11)-\mathrm{H}(11 \mathrm{~A})$ & 0.9800 \\
\hline $\mathrm{C}(16)-\mathrm{C}(17)$ & $1.515(6)$ & $\mathrm{C}(11)-\mathrm{H}(11 \mathrm{~B})$ & 0.9800 \\
\hline $\mathrm{C}(3)-\mathrm{H}(3 \mathrm{~A})$ & 0.9800 & $\mathrm{C}(11)-\mathrm{H}(11 \mathrm{C})$ & 0.9800 \\
\hline $\mathrm{C}(3)-\mathrm{H}(3 \mathrm{~B})$ & 0.9800 & $\mathrm{C}(1)-\mathrm{H}(1 \mathrm{~A})$ & 0.9800 \\
\hline $\mathrm{C}(3)-\mathrm{H}(3 \mathrm{C})$ & 0.9800 & $\mathrm{C}(1)-\mathrm{H}(1 \mathrm{~B})$ & 0.9800 \\
\hline $\mathrm{C}(15)-\mathrm{H}(15)$ & 0.9500 & $\mathrm{C}(1)-\mathrm{H}(1 \mathrm{C})$ & 0.9800 \\
\hline $\mathrm{C}(7)-\mathrm{C}(6)$ & $1.404(6)$ & $\mathrm{C}(18)-\mathrm{H}(18 \mathrm{~A})$ & 0.9800 \\
\hline $\mathrm{C}(7)-\mathrm{C}(8)$ & $1.513(6)$ & $\mathrm{C}(18)-\mathrm{H}(18 \mathrm{~B})$ & 0.9800 \\
\hline $\mathrm{C}(6)-\mathrm{H}(6)$ & 0.9500 & $\mathrm{C}(18)-\mathrm{H}(18 \mathrm{C})$ & 0.9800 \\
\hline
\end{tabular}




\begin{tabular}{|c|c|}
\hline $\mathrm{O}(1)-\mathrm{Al}(2)-\mathrm{N}(6)$ & $116.01(16)$ \\
\hline $\mathrm{O}(1)-\mathrm{Al}(2)-\mathrm{N}(5)$ & $111.28(15)$ \\
\hline $\mathrm{O}(1)-\mathrm{Al}(2)-\mathrm{N}(4)$ & $113.74(17)$ \\
\hline $\mathrm{N}(6)-\mathrm{Al}(2)-\mathrm{N}(5)$ & $96.02(15)$ \\
\hline $\mathrm{N}(4)-\mathrm{Al}(2)-\mathrm{N}(6)$ & $106.64(17)$ \\
\hline $\mathrm{N}(4)-\mathrm{Al}(2)-\mathrm{N}(5)$ & $111.81(17)$ \\
\hline $\mathrm{O}(1)-\mathrm{Al}(1)-\mathrm{N}(3)$ & $113.03(16)$ \\
\hline $\mathrm{O}(1)-\mathrm{Al}(1)-\mathrm{N}(2)$ & $112.90(16)$ \\
\hline $\mathrm{O}(1)-\mathrm{Al}(1)-\mathrm{N}(1)$ & $114.49(17)$ \\
\hline $\mathrm{N}(3)-\mathrm{Al}(1)-\mathrm{N}(2)$ & $95.70(15)$ \\
\hline $\mathrm{N}(1)-\mathrm{Al}(1)-\mathrm{N}(3)$ & $109.64(17)$ \\
\hline $\mathrm{N}(1)-\mathrm{Al}(1)-\mathrm{N}(2)$ & $109.52(16)$ \\
\hline $\mathrm{Al}(2)-\mathrm{O}(1)-\mathrm{Al}(1)$ & $175.1(2)$ \\
\hline $\mathrm{C}(7)-\mathrm{N}(3)-\mathrm{Al}(1)$ & $124.1(3)$ \\
\hline $\mathrm{C}(7)-\mathrm{N}(3)-\mathrm{C}(9)$ & $118.4(4)$ \\
\hline $\mathrm{C}(9)-\mathrm{N}(3)-\mathrm{Al}(1)$ & $117.5(3)$ \\
\hline $\mathrm{C}(4)-\mathrm{N}(2)-\mathrm{Al}(1)$ & $124.0(3)$ \\
\hline $\mathrm{C}(4)-\mathrm{N}(2)-\mathrm{C}(3)$ & $118.8(3)$ \\
\hline $\mathrm{C}(3)-\mathrm{N}(2)-\mathrm{Al}(1)$ & $116.8(3)$ \\
\hline $\mathrm{C}(16)-\mathrm{N}(6)-\mathrm{Al}(2)$ & $123.5(3)$ \\
\hline $\mathrm{C}(16)-\mathrm{N}(6)-\mathrm{C}(18)$ & $119.2(4)$ \\
\hline $\mathrm{C}(18)-\mathrm{N}(6)-\mathrm{Al}(2)$ & $116.7(3)$ \\
\hline $\mathrm{C}(13)-\mathrm{N}(5)-\mathrm{Al}(2)$ & $124.4(3)$ \\
\hline $\mathrm{C}(13)-\mathrm{N}(5)-\mathrm{C}(12)$ & $118.5(3)$ \\
\hline $\mathrm{C}(12)-\mathrm{N}(5)-\mathrm{Al}(2)$ & $116.8(3)$ \\
\hline $\mathrm{C}(10)-\mathrm{N}(4)-\mathrm{Al}(2)$ & $125.8(3)$ \\
\hline $\mathrm{C}(10)-\mathrm{N}(4)-\mathrm{C}(11)$ & $112.0(4)$ \\
\hline $\mathrm{C}(11)-\mathrm{N}(4)-\mathrm{Al}(2)$ & $122.2(3)$ \\
\hline $\mathrm{C}(2)-\mathrm{N}(1)-\mathrm{Al}(1)$ & $120.7(3)$ \\
\hline $\mathrm{C}(1)-\mathrm{N}(1)-\mathrm{Al}(1)$ & $127.4(3)$ \\
\hline $\mathrm{C}(1)-\mathrm{N}(1)-\mathrm{C}(2)$ & $111.9(4)$ \\
\hline $\mathrm{N}(2)-\mathrm{C}(4)-\mathrm{C}(6)$ & $122.2(4)$ \\
\hline $\mathrm{N}(2)-\mathrm{C}(4)-\mathrm{C}(5)$ & $120.1(4)$ \\
\hline $\mathrm{C}(6)-\mathrm{C}(4)-\mathrm{C}(5)$ & $117.7(4)$ \\
\hline $\mathrm{N}(5)-\mathrm{C}(13)-\mathrm{C}(15)$ & $121.6(4)$ \\
\hline
\end{tabular}

\begin{tabular}{|c|c|}
\hline $\mathrm{N}(5)-\mathrm{C}(13)-\mathrm{C}(14)$ & $119.8(4)$ \\
\hline $\mathrm{C}(15)-\mathrm{C}(13)-\mathrm{C}(14)$ & $118.7(4)$ \\
\hline $\mathrm{N}(6)-\mathrm{C}(16)-\mathrm{C}(15)$ & $123.2(4)$ \\
\hline $\mathrm{N}(6)-\mathrm{C}(16)-\mathrm{C}(17)$ & $119.9(4)$ \\
\hline $\mathrm{C}(15)-\mathrm{C}(16)-\mathrm{C}(17)$ & $116.9(4)$ \\
\hline $\mathrm{N}(2)-\mathrm{C}(3)-\mathrm{H}(3 \mathrm{~A})$ & 109.5 \\
\hline $\mathrm{N}(2)-\mathrm{C}(3)-\mathrm{H}(3 \mathrm{~B})$ & 109.5 \\
\hline $\mathrm{N}(2)-\mathrm{C}(3)-\mathrm{H}(3 \mathrm{C})$ & 109.5 \\
\hline $\mathrm{H}(3 \mathrm{~A})-\mathrm{C}(3)-\mathrm{H}(3 \mathrm{~B})$ & 109.5 \\
\hline $\mathrm{H}(3 \mathrm{~A})-\mathrm{C}(3)-\mathrm{H}(3 \mathrm{C})$ & 109.5 \\
\hline $\mathrm{H}(3 \mathrm{~B})-\mathrm{C}(3)-\mathrm{H}(3 \mathrm{C})$ & 109.5 \\
\hline $\mathrm{C}(13)-\mathrm{C}(15)-\mathrm{H}(15)$ & 116.3 \\
\hline $\mathrm{C}(16)-\mathrm{C}(15)-\mathrm{C}(13)$ & $127.5(4)$ \\
\hline $\mathrm{C}(16)-\mathrm{C}(15)-\mathrm{H}(15)$ & 116.3 \\
\hline $\mathrm{N}(3)-\mathrm{C}(7)-\mathrm{C}(6)$ & $122.7(4)$ \\
\hline $\mathrm{N}(3)-\mathrm{C}(7)-\mathrm{C}(8)$ & $119.8(4)$ \\
\hline $\mathrm{C}(6)-\mathrm{C}(7)-\mathrm{C}(8)$ & $117.5(4)$ \\
\hline $\mathrm{C}(4)-\mathrm{C}(6)-\mathrm{H}(6)$ & 116.5 \\
\hline $\mathrm{C}(7)-\mathrm{C}(6)-\mathrm{C}(4)$ & $127.0(4)$ \\
\hline $\mathrm{C}(7)-\mathrm{C}(6)-\mathrm{H}(6)$ & 116.5 \\
\hline $\mathrm{N}(5)-\mathrm{C}(12)-\mathrm{H}(12 \mathrm{~A})$ & 109.5 \\
\hline $\mathrm{N}(5)-\mathrm{C}(12)-\mathrm{H}(12 \mathrm{~B})$ & 109.5 \\
\hline $\mathrm{N}(5)-\mathrm{C}(12)-\mathrm{H}(12 \mathrm{C})$ & 109.5 \\
\hline $\mathrm{H}(12 \mathrm{~A})-\mathrm{C}(12)-\mathrm{H}(12 \mathrm{~B})$ & 109.5 \\
\hline $\mathrm{H}(12 \mathrm{~A})-\mathrm{C}(12)-\mathrm{H}(12 \mathrm{C})$ & 109.5 \\
\hline $\mathrm{H}(12 \mathrm{~B})-\mathrm{C}(12)-\mathrm{H}(12 \mathrm{C})$ & 109.5 \\
\hline $\mathrm{N}(1)-\mathrm{C}(2)-\mathrm{H}(2 \mathrm{~A})$ & 109.5 \\
\hline $\mathrm{N}(1)-\mathrm{C}(2)-\mathrm{H}(2 \mathrm{~B})$ & 109.5 \\
\hline $\mathrm{N}(1)-\mathrm{C}(2)-\mathrm{H}(2 \mathrm{C})$ & 109.5 \\
\hline $\mathrm{H}(2 \mathrm{~A})-\mathrm{C}(2)-\mathrm{H}(2 \mathrm{~B})$ & 109.5 \\
\hline $\mathrm{H}(2 \mathrm{~A})-\mathrm{C}(2)-\mathrm{H}(2 \mathrm{C})$ & 109.5 \\
\hline $\mathrm{H}(2 \mathrm{~B})-\mathrm{C}(2)-\mathrm{H}(2 \mathrm{C})$ & 109.5 \\
\hline $\mathrm{N}(3)-\mathrm{C}(9)-\mathrm{H}(9 \mathrm{~A})$ & 109.5 \\
\hline $\mathrm{N}(3)-\mathrm{C}(9)-\mathrm{H}(9 \mathrm{~B})$ & 109.5 \\
\hline $\mathrm{N}(3)-\mathrm{C}(9)-\mathrm{H}(9 \mathrm{C})$ & 109.5 \\
\hline $\mathrm{H}(9 \mathrm{~A})-\mathrm{C}(9)-\mathrm{H}(9 \mathrm{~B})$ & 109.5 \\
\hline
\end{tabular}




$\begin{array}{llll}\mathrm{H}(9 \mathrm{~A})-\mathrm{C}(9)-\mathrm{H}(9 \mathrm{C}) & 109.5 & \mathrm{H}(8 \mathrm{~B})-\mathrm{C}(8)-\mathrm{H}(8 \mathrm{C}) & 109.5 \\ \mathrm{H}(9 \mathrm{~B})-\mathrm{C}(9)-\mathrm{H}(9 \mathrm{C}) & 109.5 & \mathrm{C}(16)-\mathrm{C}(17)-\mathrm{H}(17 \mathrm{~A}) & 109.5 \\ \mathrm{C}(4)-\mathrm{C}(5)-\mathrm{H}(5 \mathrm{~A}) & 109.5 & \mathrm{C}(16)-\mathrm{C}(17)-\mathrm{H}(17 \mathrm{~B}) & 109.5 \\ \mathrm{C}(4)-\mathrm{C}(5)-\mathrm{H}(5 \mathrm{~B}) & 109.5 & \mathrm{C}(16)-\mathrm{C}(17)-\mathrm{H}(17 \mathrm{C}) & 109.5 \\ \mathrm{C}(4)-\mathrm{C}(5)-\mathrm{H}(5 \mathrm{C}) & 109.5 & \mathrm{H}(17 \mathrm{~A})-\mathrm{C}(17)-\mathrm{H}(17 \mathrm{~B}) & 109.5 \\ \mathrm{H}(5 \mathrm{~A})-\mathrm{C}(5)-\mathrm{H}(5 \mathrm{~B}) & 109.5 & \mathrm{H}(17 \mathrm{~A})-\mathrm{C}(17)-\mathrm{H}(17 \mathrm{C}) & 109.5 \\ \mathrm{H}(5 \mathrm{~A})-\mathrm{C}(5)-\mathrm{H}(5 \mathrm{C}) & 109.5 & \mathrm{H}(17 \mathrm{~B})-\mathrm{C}(17)-\mathrm{H}(17 \mathrm{C}) & 109.5 \\ \mathrm{H}(5 \mathrm{~B})-\mathrm{C}(5)-\mathrm{H}(5 \mathrm{C}) & 109.5 & \mathrm{~N}(4)-\mathrm{C}(11)-\mathrm{H}(11 \mathrm{~A}) & 109.5 \\ \mathrm{C}(13)-\mathrm{C}(14)-\mathrm{H}(14 \mathrm{~A}) & 109.5 & \mathrm{~N}(4)-\mathrm{C}(11)-\mathrm{H}(11 \mathrm{~B}) & 109.5 \\ \mathrm{C}(13)-\mathrm{C}(14)-\mathrm{H}(14 \mathrm{~B}) & 109.5 & \mathrm{~N}(4)-\mathrm{C}(11)-\mathrm{H}(11 \mathrm{C}) & 109.5 \\ \mathrm{C}(13)-\mathrm{C}(14)-\mathrm{H}(14 \mathrm{C}) & 109.5 & \mathrm{H}(11 \mathrm{~A})-\mathrm{C}(11)-\mathrm{H}(11 \mathrm{~B}) & 109.5 \\ \mathrm{H}(14 \mathrm{~A})-\mathrm{C}(14)-\mathrm{H}(14 \mathrm{~B}) & 109.5 & \mathrm{H}(11 \mathrm{~A})-\mathrm{C}(11)-\mathrm{H}(11 \mathrm{C}) & 109.5 \\ \mathrm{H}(14 \mathrm{~A})-\mathrm{C}(14)-\mathrm{H}(14 \mathrm{C}) & 109.5 & \mathrm{H}(11 \mathrm{~B})-\mathrm{C}(11)-\mathrm{H}(11 \mathrm{C}) & 109.5 \\ \mathrm{H}(14 \mathrm{~B})-\mathrm{C}(14)-\mathrm{H}(14 \mathrm{C}) & 109.5 & \mathrm{~N}(1)-\mathrm{C}(1)-\mathrm{H}(1 \mathrm{~A}) & 109.5 \\ \text { N(4)-C(10)-H(10A) } & 109.5 & \mathrm{~N}(1)-\mathrm{C}(1)-\mathrm{H}(1 \mathrm{~B}) & 109.5 \\ \mathrm{~N}(4)-\mathrm{C}(10)-\mathrm{H}(10 \mathrm{~B}) & 109.5 & \mathrm{~N}(1)-\mathrm{C}(1)-\mathrm{H}(1 \mathrm{C}) & 109.5 \\ \text { N(4)-C(10)-H(10C) } & 109.5 & \mathrm{H}(1 \mathrm{~A})-\mathrm{C}(1)-\mathrm{H}(1 \mathrm{~B}) & 109.5 \\ \mathrm{H}(10 \mathrm{~A})-\mathrm{C}(10)-\mathrm{H}(10 \mathrm{~B}) & 109.5 & \mathrm{H}(1 \mathrm{~A})-\mathrm{C}(1)-\mathrm{H}(1 \mathrm{C}) & 109.5 \\ \mathrm{H}(10 \mathrm{~A})-\mathrm{C}(10)-\mathrm{H}(10 \mathrm{C}) & 109.5 & \mathrm{H}(1 \mathrm{~B})-\mathrm{C}(1)-\mathrm{H}(1 \mathrm{C}) & 109.5 \\ \mathrm{H}(10 \mathrm{~B})-\mathrm{C}(10)-\mathrm{H}(10 \mathrm{C}) & 109.5 & \mathrm{~N}(6)-\mathrm{C}(18)-\mathrm{H}(18 \mathrm{~A}) & 109.5 \\ \mathrm{C}(7)-\mathrm{C}(8)-\mathrm{H}(8 \mathrm{~A}) & 109.5 & \mathrm{~N}(6)-\mathrm{C}(18)-\mathrm{H}(18 \mathrm{~B}) & 109.5 \\ \mathrm{C}(7)-\mathrm{C}(8)-\mathrm{H}(8 \mathrm{~B}) & 109.5 & \mathrm{~N}(6)-\mathrm{C}(18)-\mathrm{H}(18 \mathrm{C}) & 109.5 \\ \mathrm{C}(7)-\mathrm{C}(8)-\mathrm{H}(8 \mathrm{C}) & 109.5 & \mathrm{H}(18 \mathrm{~A})-\mathrm{C}(18)-\mathrm{H}(18 \mathrm{C}) & 109.5 \\ \mathrm{H}(8 \mathrm{~A})-\mathrm{C}(8)-\mathrm{H}(8 \mathrm{~B}) & 109.5 & & 109.5 \\ \mathrm{H}(8 \mathrm{~A})-\mathrm{C}(8)-\mathrm{H}(8 \mathrm{C}) & 109.5 & & \\ & & 18)-\mathrm{H}(18 \mathrm{~B}) & 109.5 \\ & & & \end{array}$


Table 5.20: Anisotropic displacement parameters $\left(\AA^{2} \times 10^{3}\right)$ for 6 . The anisotropic displacement factor exponent takes the form: $-2 \pi^{2}\left[\mathrm{~h}^{2} \mathrm{a}^{* 2} \mathrm{U}^{11}+\ldots+2 \mathrm{~h} \mathrm{k} \mathrm{a}^{*} \mathrm{~b}^{*}\right.$ $\left.\mathrm{U}^{12}\right]$.

\begin{tabular}{|c|c|c|c|c|c|c|}
\hline & $\mathrm{U}^{11}$ & $\mathrm{U}^{22}$ & $\mathrm{U}^{33}$ & $\mathrm{U}^{23}$ & $\mathrm{U}^{13}$ & $\mathrm{U}^{12}$ \\
\hline $\operatorname{Al}(2)$ & $31(1)$ & $25(1)$ & $23(1)$ & $-1(1)$ & $-3(1)$ & $0(1)$ \\
\hline $\operatorname{Al}(1)$ & $29(1)$ & $27(1)$ & $24(1)$ & $-3(1)$ & $-1(1)$ & $-1(1)$ \\
\hline $\mathrm{O}(1)$ & $44(2)$ & $34(2)$ & $24(2)$ & $-2(1)$ & $-3(1)$ & $-2(1)$ \\
\hline $\mathrm{N}(3)$ & $31(2)$ & $26(2)$ & $27(2)$ & $-1(2)$ & $2(2)$ & $-3(2)$ \\
\hline $\mathrm{N}(2)$ & $28(2)$ & $22(2)$ & $27(2)$ & $-3(1)$ & $-1(2)$ & $-1(1)$ \\
\hline $\mathrm{N}(6)$ & $31(2)$ & $27(2)$ & $31(2)$ & $-2(2)$ & $-6(2)$ & $0(2)$ \\
\hline $\mathrm{N}(5)$ & $33(2)$ & $24(2)$ & $26(2)$ & $1(1)$ & $-1(2)$ & $-1(2)$ \\
\hline $\mathrm{N}(4)$ & $34(2)$ & $38(2)$ & $29(2)$ & $-3(2)$ & $-2(2)$ & $-4(2)$ \\
\hline $\mathrm{N}(1)$ & $29(2)$ & $41(2)$ & $33(2)$ & $-5(2)$ & $-3(2)$ & $1(2)$ \\
\hline $\mathrm{C}(4)$ & $27(2)$ & $32(2)$ & $24(2)$ & $-7(2)$ & $0(2)$ & $4(2)$ \\
\hline $\mathrm{C}(13)$ & $31(2)$ & $34(2)$ & $28(2)$ & $-2(2)$ & $4(2)$ & $-7(2)$ \\
\hline $\mathrm{C}(16)$ & $26(2)$ & $40(3)$ & $25(2)$ & $7(2)$ & $-1(2)$ & $-3(2)$ \\
\hline $\mathrm{C}(3)$ & $35(3)$ & $29(2)$ & $34(3)$ & $0(2)$ & $-2(2)$ & $-4(2)$ \\
\hline $\mathrm{C}(15)$ & $35(3)$ & $37(3)$ & $22(2)$ & $-1(2)$ & $-6(2)$ & $-8(2)$ \\
\hline $\mathrm{C}(7)$ & $31(2)$ & $32(2)$ & $31(2)$ & $7(2)$ & $5(2)$ & $2(2)$ \\
\hline $\mathrm{C}(6)$ & $38(3)$ & $37(2)$ & $21(2)$ & $2(2)$ & $-2(2)$ & $1(2)$ \\
\hline $\mathrm{C}(12)$ & $40(3)$ & $30(2)$ & $36(3)$ & $1(2)$ & $-3(2)$ & $5(2)$ \\
\hline $\mathrm{C}(2)$ & $34(3)$ & $33(2)$ & $44(3)$ & $-7(2)$ & $7(2)$ & $-3(2)$ \\
\hline $\mathrm{C}(9)$ & $37(3)$ & $30(2)$ & $41(3)$ & $-5(2)$ & $5(2)$ & $-7(2)$ \\
\hline $\mathrm{C}(5)$ & $35(3)$ & $38(3)$ & $38(3)$ & $-9(2)$ & $-6(2)$ & $-2(2)$ \\
\hline $\mathrm{C}(14)$ & $52(3)$ & $35(3)$ & $32(3)$ & $-5(2)$ & $3(2)$ & $-12(2)$ \\
\hline $\mathrm{C}(10)$ & $31(3)$ & $37(3)$ & $55(3)$ & $-2(2)$ & $-15(2)$ & $-2(2)$ \\
\hline $\mathrm{C}(8)$ & $51(3)$ & $34(3)$ & $43(3)$ & $12(2)$ & $5(2)$ & $0(2)$ \\
\hline $\mathrm{C}(17)$ & $42(3)$ & $52(3)$ & $38(3)$ & $20(2)$ & $-8(2)$ & $-2(2)$ \\
\hline $\mathrm{C}(11)$ & $41(3)$ & $38(3)$ & $41(3)$ & $-6(2)$ & $6(2)$ & $-3(2)$ \\
\hline $\mathrm{C}(1)$ & $33(3)$ & $42(3)$ & $53(3)$ & $4(2)$ & $-12(2)$ & $-1(2)$ \\
\hline $\mathrm{C}(18)$ & $48(3)$ & $35(3)$ & $47(3)$ & $2(2)$ & $-4(2)$ & $6(2)$ \\
\hline
\end{tabular}


Table 5.21: Hydrogen coordinates (x $10^{4}$ ) and isotropic displacement parameters $\left(\AA^{2} \times 10^{3}\right)$ for 6.

\begin{tabular}{|c|c|c|c|c|}
\hline & $\mathrm{x}$ & $\mathrm{y}$ & $\mathrm{z}$ & $\mathrm{U}(\mathrm{eq})$ \\
\hline $\mathrm{H}(3 \mathrm{~A})$ & 4015 & 5024 & 816 & 49 \\
\hline $\mathrm{H}(3 \mathrm{~B})$ & 2508 & 5348 & 1331 & 49 \\
\hline $\mathrm{H}(3 \mathrm{C})$ & 4140 & 5312 & 2034 & 49 \\
\hline $\mathrm{H}(15)$ & 7463 & 6979 & 7185 & 38 \\
\hline $\mathrm{H}(6)$ & 3700 & 6577 & -1552 & 38 \\
\hline $\mathrm{H}(12 \mathrm{~A})$ & 4254 & 7472 & 3543 & 53 \\
\hline $\mathrm{H}(12 \mathrm{~B})$ & 5590 & 7897 & 4014 & 53 \\
\hline $\mathrm{H}(12 \mathrm{C})$ & 3954 & 7815 & 4685 & 53 \\
\hline $\mathrm{H}(2 \mathrm{~A})$ & 7434 & 6031 & -422 & 55 \\
\hline $\mathrm{H}(2 \mathrm{~B})$ & 8585 & 5527 & -21 & 55 \\
\hline $\mathrm{H}(2 \mathrm{C})$ & 9223 & 6166 & -27 & 55 \\
\hline $\mathrm{H}(9 \mathrm{~A})$ & 5342 & 7762 & 1353 & 54 \\
\hline $\mathrm{H}(9 \mathrm{~B})$ & 6951 & 7664 & 660 & 54 \\
\hline $\mathrm{H}(9 \mathrm{C})$ & 6774 & 7405 & 1899 & 54 \\
\hline $\mathrm{H}(5 \mathrm{~A})$ & 3163 & 5250 & -1020 & 56 \\
\hline $\mathrm{H}(5 \mathrm{~B})$ & 2317 & 5775 & -1633 & 56 \\
\hline $\mathrm{H}(5 \mathrm{C})$ & 1639 & 5544 & -461 & 56 \\
\hline $\mathrm{H}(14 \mathrm{~A})$ & 6681 & 8131 & 5710 & 59 \\
\hline $\mathrm{H}(14 \mathrm{~B})$ & 6854 & 7901 & 6974 & 59 \\
\hline $\mathrm{H}(14 \mathrm{C})$ & 5120 & 7997 & 6434 & 59 \\
\hline $\mathrm{H}(10 \mathrm{~A})$ & 936 & 6248 & 4282 & 62 \\
\hline $\mathrm{H}(10 \mathrm{~B})$ & 1575 & 5613 & 4146 & 62 \\
\hline $\mathrm{H}(10 \mathrm{C})$ & 2132 & 6094 & 3281 & 62 \\
\hline $\mathrm{H}(8 \mathrm{~A})$ & 4104 & 7824 & -542 & 64 \\
\hline $\mathrm{H}(8 \mathrm{~B})$ & 4368 & 7489 & -1698 & 64 \\
\hline $\mathrm{H}(8 \mathrm{C})$ & 5874 & 7705 & -983 & 64 \\
\hline $\mathrm{H}(17 \mathrm{~A})$ & 7665 & 5644 & 7303 & 66 \\
\hline $\mathrm{H}(17 \mathrm{~B})$ & 8741 & 6188 & 7571 & 66 \\
\hline $\mathrm{H}(17 \mathrm{C})$ & 9199 & 5777 & 6550 & 66 \\
\hline $\mathrm{H}(11 \mathrm{~A})$ & 3767 & 6160 & 6444 & 60 \\
\hline $\mathrm{H}(11 \mathrm{~B})$ & 2611 & 5648 & 6101 & 60 \\
\hline $\mathrm{H}(11 \mathrm{C})$ & 1917 & 6277 & 6195 & 60 \\
\hline
\end{tabular}




\begin{tabular}{lllll}
$\mathrm{H}(1 \mathrm{~A})$ & 8585 & 5919 & 2827 & 64 \\
$\mathrm{H}(1 \mathrm{~B})$ & 9951 & 5975 & 1897 & 64 \\
$\mathrm{H}(1 \mathrm{C})$ & 8998 & 5392 & 2034 & 64 \\
$\mathrm{H}(18 \mathrm{~A})$ & 6613 & 5223 & 5594 & 65 \\
$\mathrm{H}(18 \mathrm{~B})$ & 8197 & 5414 & 4949 & 65 \\
$\mathrm{H}(18 \mathrm{C})$ & 6545 & 5360 & 4274 & 65 \\
\hline
\end{tabular}




\title{
Chapter 6
}

\section{A Low Cost, High Efficiency TMA-}

\section{Replacement for the Deposition of Pure}

\author{
Aluminum Nitride by Atomic Layer
}

\section{Deposition}

A manuscript for the following work is in preparation. Co-contributors to this work include Polla Rouf ${ }^{b}$, Petro Deminskyi ${ }^{b}$, Seán T. Barry and Henrik Pedersen ${ }^{b}$.

aDepartment of Chemistry, Carleton University, Ottawa, Ontario K1S 5B6, Canada

bDepartment of Physics, Chemistry, and Biology, Linköping University, Linköping SE-58158, Sweden 


\subsection{Abstract}

A heteroleptic aluminum hydride/amide precursor is presented as a viable candidate to replace trimethylaluminum (TMA) for atomic layer deposition of aluminum nitride $(\mathrm{AlN})$. Ammonia $\left(\mathrm{NH}_{3}\right)$ plasma was used as a co-reagent to produce AlN films, which were crystalline when deposited at temperatures at 200 ${ }^{\circ} \mathrm{C}$ and above. The films produced were uniform and stoichiometric with an Al:N ratio of 1.04. By designing a simple, low cost precursor $\left(\mathrm{AlH}_{2}\left(\mathrm{NMe}_{2}\right)\right)$ with only metal-nitrogen and metal-hydrogen bonds, carbon and oxygen impurities in resultant AlN films were reduced to $<1 \%$ and $<2 \%$, respectively. Compared to TMA, this precursor can improve film quality and reduce material cost by up to 2 orders of magnitude.

\subsection{Introduction}

Aluminum nitride is an important wide bandgap $(6.2 \mathrm{eV})$ semiconductor for use in microelectronics, which has applications as a dielectric layer and for use in lightemitting diodes. ${ }^{1}$ Due to increasingly small and non-trivial device morphologies, atomic layer deposition (ALD) is a valuable thin film deposition technique to employ for next generation microelectronic devices. ALD is a subset of chemical vapour deposition (CVD), but distinctly only employs surface-limited and self- 
terminating chemical reactions, allowing precise thickness control down to the Ångström level.

TMA is a very commonly used precursor to deposit $\mathrm{Al}_{2} \mathrm{O}_{3}$ and $\mathrm{AlN}$, but it has a drawback: many processes report carbon impurities in deposited films on the order of 3-6\%; ${ }^{37,97,100}$ these carbon impurities originate from the direct metal-carbon bonds present in this precursor. ${ }^{37}$ Though TMA is readily available and wellstudied, it does not deposit films with sufficiently low impurities, and these levels can be improved through precursor design.

The basis behind this research emphasizes the importance of ligand-metal bonding in a precursor. Specifically, the inclusion of metal-nitrogen bonds and the elimination of metal-carbon bonds is an intuitive way to deposit 13-nitride films with low $\mathrm{C}$ contamination. We initially used TMA as a template to design an analogue, replacing all methyl groups with amide ligands; tris(dimethylamido)aluminum(III) was used to deposit aluminum oxide by ALD with carbon contamination of less than 1\%.75 The evolved precursor design presented herein came from an effort to minimize oxygen incorporation during film growth. Using ligand design to our advantage, the hydride ligand was employed to produce $\mathrm{AlH}_{2}\left(\mathrm{NMe}_{2}\right)$ (9). We hypothesized that this precursor could provide reducing surface conditions to allow the growing AlN film to resist oxidation. 


\subsection{Experimental}

Compound 9 was synthesized in a nitrogen-filled glovebox in a method adapted from Ruff and Hawthorne. ${ }^{242}$ Dimethylammonium chloride was dried under vacuum and all solvents were purified and dried over $4 \AA$ sieves. $\mathrm{LiAlH}_{4}$ (4.119 g, $109 \mathrm{mmol}$ ) was added to a 250 round-bottomed flask with $\sim 75 \mathrm{~mL}$ diethyl ether and stirred with a magnetic stir bar. $\mathrm{Me}_{2} \mathrm{NH}_{2} \mathrm{Cl}(8.595 \mathrm{~g}, 105 \mathrm{mmol})$ was cooled ($35{ }^{\circ} \mathrm{C}$ ), then added scoop-wise to the $\mathrm{LiAlH}_{4}$ suspension. The mixture bubbled vigorously upon sequential additions and the resultant solution stirred overnight. The solution was filtered through a medium fritted filter and the volume was reduced under vacuum to produce a white solid (6.248 g, $81 \%$ unpurified yield).

Thermogravimetric analysis (TGA) experiments were performed using a TGA Q500 tool in an $\mathrm{N}_{2}$ filled glovebox. The sample Pt pan was loaded with $\sim 10$ mg of precursor and the furnace was heated at a rate of $10{ }^{\circ} \mathrm{C} / \mathrm{min}$ to $500{ }^{\circ} \mathrm{C}$.

ALD experiments were performed on a hot-wall Picosun R-200 tool with a Litmas remote plasma source. The operating pressure was maintained at 4 mbar under a continuous flow of $\mathrm{N}_{2}(99.999 \%)$, which was also used as a purge gas. Ammonia plasma as the co-reagent was pulsed for $12 \mathrm{~s}$ at a power of $2800 \mathrm{~W}$. AlN films were deposited on $2.5 \mathrm{~cm} \times 2.5 \mathrm{~cm} \mathrm{Si}$ coupons. 
PANalytical EMPYREAN MRD XRD with a Cu-anode X-ray tube and 5axis (x-y-z-v-u) sample stage operating at $45 \mathrm{kV}$ and $40 \mathrm{~mA}$ was used in grazingincidence X-ray diffraction (GIXRD) mode with $0.5^{\circ}$ incident angle to analyze film crystallinity. Stress and strain were estimated using GIXRD data for diffraction peaks; values of $\sin ^{2} \psi$ and $\varepsilon$ were obtained using X'Pert Stress software. Kratos AXIS Ultra DLD X-ray photoelectron spectroscopy (XPS) equipped with an Ar sputtering source $(0.5 \mathrm{keV})$ was used to analyze film composition. CasaXPS was used to analyze XPS data. High-resolution scans were fitted by GaussianLaurentius functions and Shirley background. Scanning electron microscopy (SEM) images were collected using a LEO 1550 tool operating at $10 \mathrm{kV}$ in coordination with energy-dispersive X-ray spectroscopy (EDS) for compositional analysis.

\subsection{Results and Discussion}

The TGA for $\mathbf{9}$ (Figure 6.1) demonstrated that it was a volatile, thermally stable precursor with no visible signs of decomposition. Its 1 Torr temperature was measured to be $40{ }^{\circ} \mathrm{C}$ using mass loss derivative data. ${ }^{20}$ 


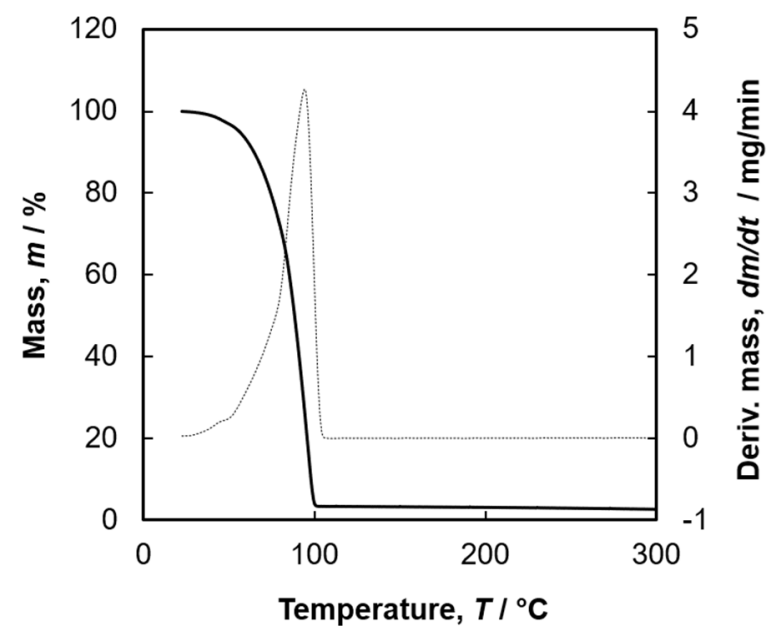

Figure 6.1: TGA ramp of compound 9.

The saturation curve for $\mathbf{9}$ in ALD experiments to produce AlN is presented in Figure 6.2a. Saturative growth is observed after $8 \mathrm{~s}$ of exposure at temperatures of 150 and $200{ }^{\circ} \mathrm{C}$ with a growth per cycle (GPC) of approximately $4 \AA$. This GPC is notably higher than previous AlN ALD reports, though still lies within the lattice parameters for hexagonal $\mathrm{AlN}^{243}$ and is therefore reasonable for this process. Experiments run at $100{ }^{\circ} \mathrm{C}$ do not exhibit saturative ALD behaviour, which can be attributed to precursor condensation at the surface.

SEM micrographs (Figure 6.2c-d) show that ALD-grown AlN films are uniform and dense. Experiments performed at $100{ }^{\circ} \mathrm{C}$ (Figure 6.2a), however, show droplets present on the surface, which confirms that precursor condenses on the substrate at lower deposition temperatures. EDS measurements of AlN deposited at $200{ }^{\circ} \mathrm{C}$ demonstrated an $\mathrm{Al}: \mathrm{N}$ ratio of 1.04 , indicating that stoichiometric AlN was deposited. 

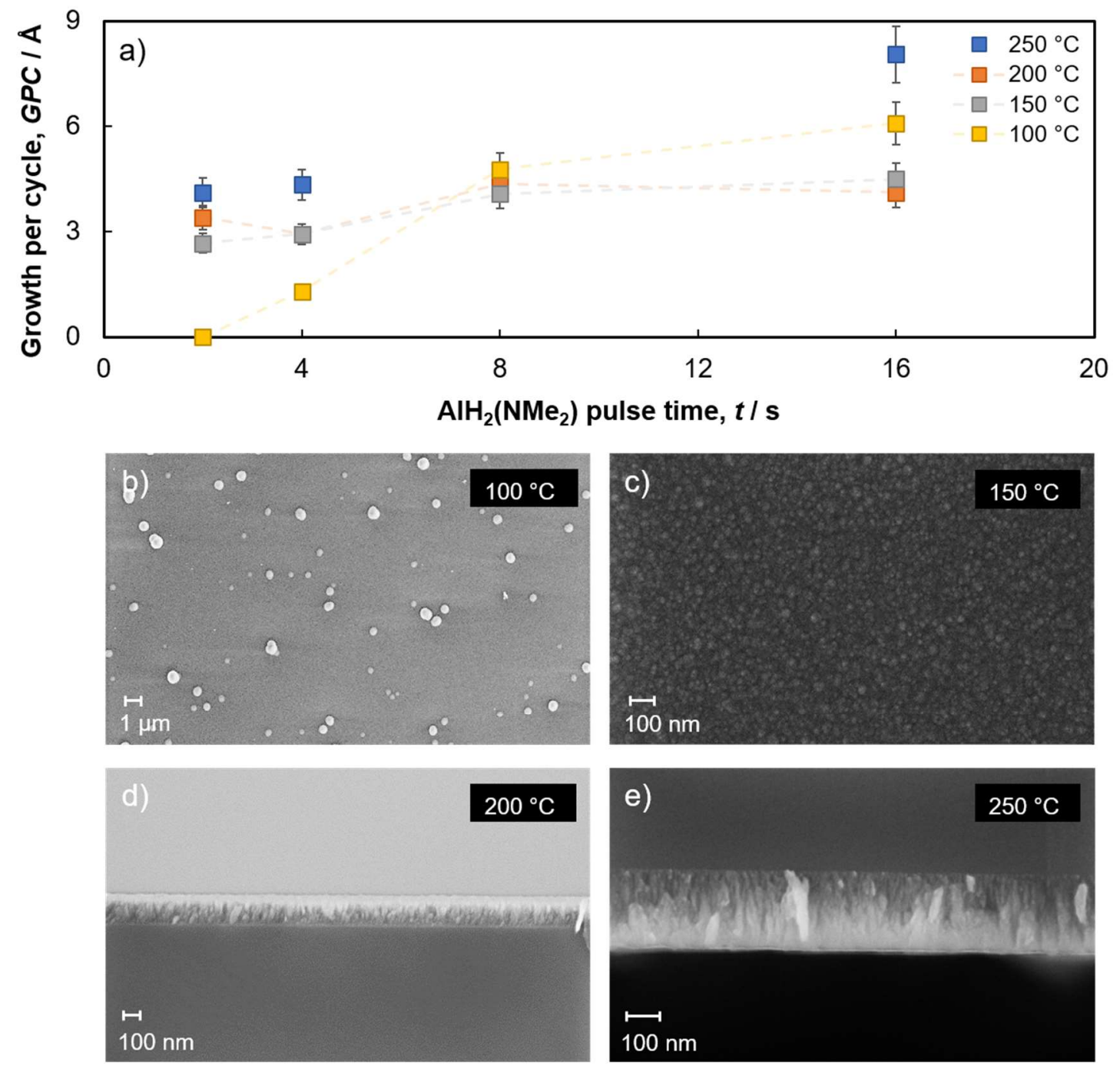

Figure 6.2: a) Saturation curve for 9 in ALD process with $\mathrm{NH}_{3}$ plasma, and SEM micrographs of AlN films deposited at b) $100{ }^{\circ} \mathrm{C}$, c) $150{ }^{\circ} \mathrm{C}$, and d) $200{ }^{\circ} \mathrm{C}$.

Figures 6.3a shows GIXRD data for AlN films deposited between $100{ }^{\circ} \mathrm{C}$ and $250{ }^{\circ} \mathrm{C}$, after both 200 and 500 cycles of deposition. Films deposited at 200 and $250{ }^{\circ} \mathrm{C}$ showed the strongest peaks corresponding to crystalline hexagonal AlN; both the (100) and (002) peaks were observed. These peaks were also visible for the samples deposited at $150{ }^{\circ} \mathrm{C}$, but with lower intensity. Crystallinity of these 
AlN films was seen to increase both with increased deposition temperature and number of cycles. Low temperature depositions $\left(100{ }^{\circ} \mathrm{C}\right)$ do not appear to be crystalline.
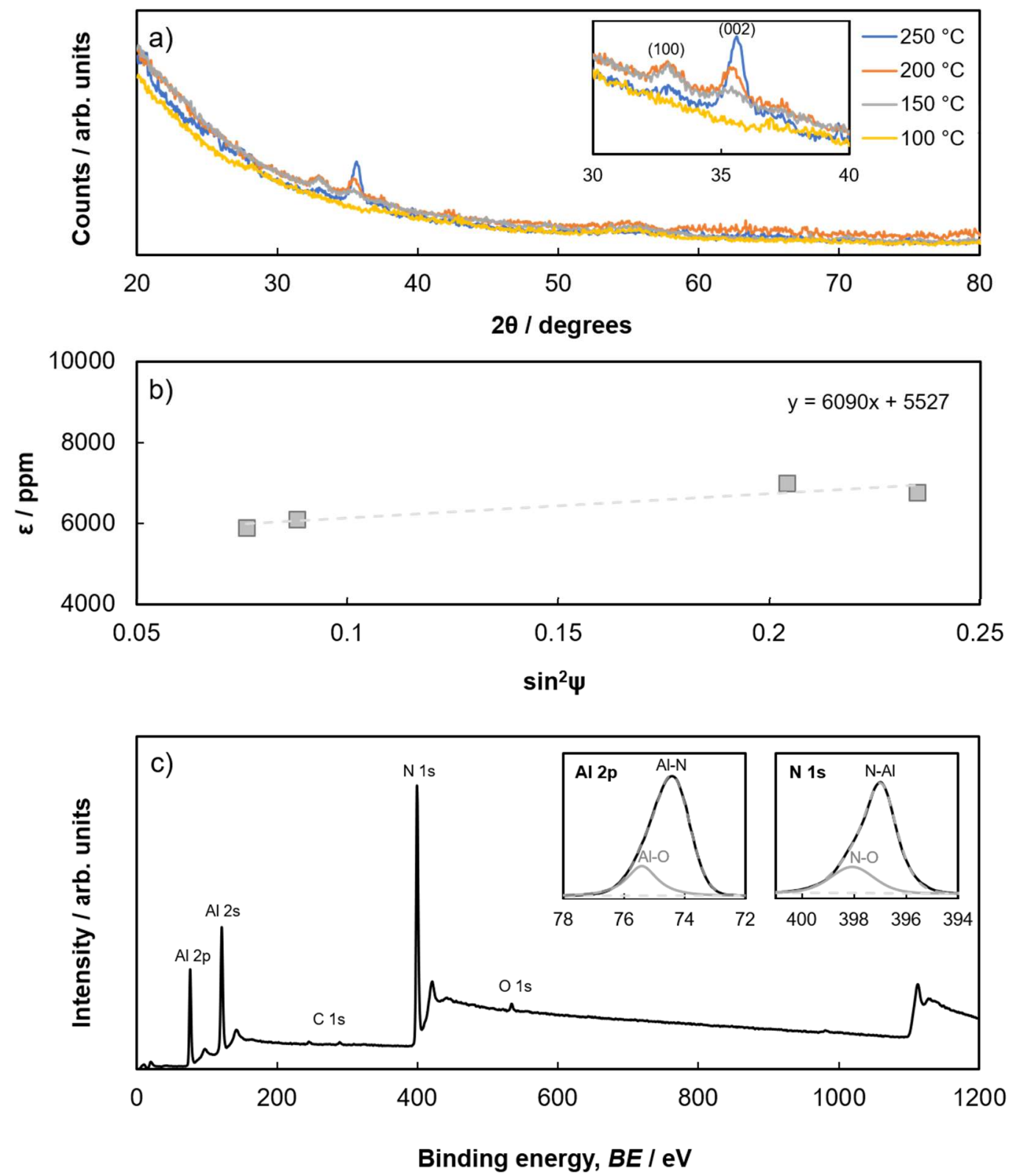

Figure 6.3: a) GIXRD plot of AlN film, b) $\sin ^{2} \psi$ vs. $\varepsilon$ plot to calculate film strain, c) XPS survey scan of AlN film deposited at $200{ }^{\circ} \mathrm{C}$ and inset high-resolution scans for $\mathrm{Al}$ and $\mathrm{N}$. AlN films were deposited using $\mathbf{9}$ and $\mathrm{NH}_{3}$ plasma. 
Film composition was studied by XPS (Figure 6.3c) after sputtering to clean incidental contamination from the surface. High-resolution peaks were fitted for $\mathrm{Al}$ $2 \mathrm{p}$ at 74.7 and $75.5 \mathrm{eV}$ as representing $\mathrm{Al}-\mathrm{N}$ and $\mathrm{Al}-\mathrm{O}$ bonds, respectively, while the $\mathrm{N}$ 1s spectrum was fitted for N-Al and N-O bonds at 397.9 and $399.4 \mathrm{eV}$. Importantly, for AlN deposited at $200{ }^{\circ} \mathrm{C}$, we observed impurity levels of carbon and oxygen at 0.7 and $1.7 \%$, respectively. This indicates that metal-nitrogen and metal-hydride bonds designed into the precursor successfully respectively reduced carbon and oxygen contamination in AlN films.

Finally, the stress and strain of the AlN films were estimated using GIXRD data from an $\varepsilon-\sin ^{2} \psi$ plot using a previously reported method, ${ }^{244}$ shown in Figure 6.3b. The positive slope of $6090 \mathrm{ppm}$ indicates a tensile strain of $0.61 \%$. Tensile stress was calculated as a range between 1.50 and $1.67 \mathrm{GPa} .{ }^{243,244} \mathrm{It}$ is expected for tensile strain and stress to exist in these AlN films due their large lattice mismatch compared to the Si substrate. Upcoming work will focus on mobility and charge carrier measurements by depositing AlN on semiconducting SiC. This substrate has a smaller lattice mismatch with AlN, and the resultant deposited films are more likely to be epitaxial. 


\subsection{Summary and conclusions}

This work emphasizes the importance of developing non-TMA precursors for Albased ALD. The new ALD precursor $\mathrm{AlH}_{2}\left(\mathrm{NMe}_{2}\right)(\mathbf{9})$ produces excellent aluminum nitride films in a process with ammonia plasma with measured carbon impurities significantly lower than many processes reported using TMA.

Using a TMA framework is an excellent way to design and synthesize alternative precursors better suited to AlN deposition by ALD. $\mathrm{AlH}_{2}\left(\mathrm{NMe}_{2}\right)(\mathbf{9})$ is easy to synthesize on a lab scale (tens of grams) in less than two hours at a materials cost of $\$ 1.26$ (CAD) per gram. On an "industry" scale (on the order of kilograms), this precursor could be synthesized for $\$ 0.67$ per gram based on given catalog prices for starting materials. This is significant to compare to the cost of TMA, which is available for purchase ( $25 \mathrm{~g}$ in a bubbler) from Sigma Aldrich at a cost of $\$ 2440$, equivalent to $\$ 62$ per gram. It is especially important to consider the growth per cycle of AlN achieved using $\mathbf{9}$, which is nearly four times higher than that when using TMA.

A simple shift away from TMA towards $\mathbf{9}$ in this experiment has shown that, if we design smart precursors, the quality of ALD-grown films and their subsequent applications can be improved; we should synthesize precursors to our advantage, and using $\mathbf{9}$ to deposit pure AlN is a demonstration of how well this can work. 


\subsection{Acknowledgements}

This project was funded by the Swedish foundation for Strategic Research through the project "Time-resolved low temperature CVD for III-nitrides" (No. SSF-RMA 15-0018). S.C.B. acknowledges the Vinnova VINNMER Marie Curie incoming mobility program for funding research visits to Linköping University (Vinnova Grant No. 2015-03714). 


\section{Chapter 7}

\section{Conclusions}

While this thesis has presented work from a variety of projects, the central focus remained on the design, development, and characterization of group 13 precursors and ALD processes with the goal of depositing low-impurity III-N thin films. This began by using a familiar ligand system in guanidinates, though its limitations were observed, and subsequent precursor design was performed as a result. By moving towards simpler amide precursors for both aluminum and gallium metal centres, it was observed that these volatile and thermally stable metal amides deposited films with reduced carbon impurities and could deposit epitaxial metal nitride thin films with the use of the appropriate co-reagent. The nitrogen precursor was also studied in an attempt to improve $13-\mathrm{N}$ deposition by CVD, though it was discovered that 
methylamines compared to ammonia had reduced reactivity at the surface. When other, more complex group 13 precursors were synthesized, they were demonstrated to be useful in depositing high quality metal nitride films, and in producing interesting and novel group 13 molecules. The use of a simple, heteroleptic aluminum hydride/amide precursor that was used to deposit pure AlN films ultimately demonstrated that precursor design is paramount in importance and has a strong influence on the purity of final deposited films by ALD.

In chapter 2, tris-N,N,-dimethyl-N',N"- diisopropylguanidinatoindium(III) was thermally evaluated in order to characterize anomalies that have been observed from its use in CVD and ALD processes. Guanidinates have been popular ligands to use for ALD precursors, however, their main drawback is that they can undergo thermal decomposition, mainly by CDI deinsertion. It was demonstrated in this chapter that this precursor thermolyzed to a bis(guanidinate) species and then to a mono(guanidinate) species; this was modelled computationally and then observed by TGA and NMR experiments. This occurred as a result of sequential CDI elimination from the precursor over longer periods of time at increased temperatures. This means that tris-N,N,-dimethyl-N',N"diisopropylguanidinatoindium(III) can be used in deposition experiments, but should not be stored in heated bubblers over multiple runs in order to minimize decomposition and deposit low-impurity thin films with straightforward surface 
chemistry. While this chemistry is interesting to study, this work demonstrates that a shift towards simpler precursors with straightforward reactivity and thermal stability are preferred for use in ALD processes.

In a shift towards simpler precursors, $\mathrm{Al}\left(\mathrm{NMe}_{2}\right)_{3}$ and $\mathrm{Ga}\left(\mathrm{NMe}_{2}\right)_{3}$ were synthesized for the deposition of group 13 nitrides by ALD in chapter 3. This work stemmed from the idea that to eliminate carbon impurities in $13-\mathrm{N}$ films that are present due to metal-carbon bonds in TMA, newly designed precursors should only have aluminum bonded directly to nitrogen. The simple aluminum amide TMA analogue is easy to synthesize and was demonstrated by TGA to be thermally stable and volatile with a 1 Torr temperature of $78^{\circ} \mathrm{C}$. An ALD process using tris(dimethylamido)aluminum(III) and water as the co-reagent demonstrated that $\mathrm{Al}_{2} \mathrm{O}_{3}$ films could be deposited at $250{ }^{\circ} \mathrm{C}$ with approximately $1 \%$ carbon impurities, which is lower than those reported for processes using TMA. Attempts to deposit AlN using nitrogen plasma as a co-reagent produced an aluminum oxynitride but maintained extremely low levels of carbon impurities (1\%). Changing the coreagent to $\mathrm{NH}_{3}$ plasma for the ALD process using $\mathrm{Ga}\left(\mathrm{NMe}_{2}\right)_{3}$ proved to be very effective and successfully deposited GaN with low carbon and oxygen impurities between 130 and $250{ }^{\circ} \mathrm{C}$ and a growth of $1.4 \AA$ per cycle. The GaN was found to be nearly stoichiometric and when deposited on $4 \mathrm{H}-\mathrm{SiC}$, the films were epitaxial. This work demonstrated that moving towards simpler precursors was beneficial not 
only due to their ease of synthesis, but more importantly due to the fact that their thermal stability and volatility improved. An indium analogue of this precursor is not accessible, but a move towards other simple N-bonded ligands to produce indium precursors for InN should be pursued in future studies.

It was demonstrated in chapter 3 that the choice of a nitrogen precursor or co-reagent is also very important to produce metal nitride films, and this was further studied by quantum chemical computations in the investigation of methylamines as nitrogen precursors for CVD of GaN in chapter 4. It is known that ammonia used in thermal CVD processes as a co-reagent requires N:13 ratios of over 100:1; this work hypothesized that replacing N-H bonds with weaker N-C bonds could reduce this factor and improve precursor chemistry in CVD processes. However, while methylamine precursors were more reactive in the gas phase than ammonia was, they only deposited micron-sized Ga droplets when used in ALD processes; no continuous GaN films were deposited using methylamine precursors. Amines decomposed more easily than ammonia, and their decomposition products likely polluted growing GaN surfaces which forced the formation of metallic Ga droplets. Ammonia was more reactive at the surface, and it is hypothesized that this surface chemistry is more important to consider than gas phase chemistry to efficiently deposit GaN by CVD. This work suggests that any future development 
of nitrogen precursors for GaN CVD should avoid the formation of highly reactive hydrocarbon fragments in order to prevent Ga droplet formation on the surface.

Bidentate ligands that still maintain metal-nitrogen bonds were investigated in chapter 5 , beginning with a new indium (III) triazenide precursor. This precursor was shown to be volatile and thermally stable by TGA with a 1 Torr temperature of $134^{\circ} \mathrm{C}$. Most interestingly, this precursor was used in an InN ALD process with ammonia plasma that showed two temperature intervals with different self-limited growth behaviour. In the context of this thesis, the thermal work particularly highlighted the existence of two distinct thermal events, which in turn explained the existence of two temperature intervals where ALD could occur. It is likely that at high temperatures, the initial precursor thermally decomposed to a more chemically reactive tricoordinated $\mathrm{In}(\mathrm{III})$ species upon the liberation of $\mathrm{N}_{2}$ and propene. The GPC values for low temperature range $\left(220-250{ }^{\circ} \mathrm{C}\right)$ and high temperature range $\left(300-350{ }^{\circ} \mathrm{C}\right)$ were $0.4 \AA /$ cycle and $1.2 \AA /$ cycle, respectively. The InN films produced were stoichiometric and were also epitaxial when grown on $4 \mathrm{H}-\mathrm{SiC}$. They had very low levels of oxygen and carbon impurities as determined by XPS. This work demonstrates the first report of a homoleptic triazenide in a vapour deposition process, and continued work is employing this ligand on other group 13 metal centres, as well as manipulating the symmetry and substitution of the azenide ligand itself. This ligand could be employed on metal centres across the 
periodic table and could be a very valuable asset in developing ALD precursors and processes. This ligand is novel to this realm of research, and its design can be exploited to deliberately decompose in the gas phase to manipulate ALD growth behaviour.

Another bidentate ligand studied in chapter 5 was the MeNacNac ligand, which was employed in heteroleptic aluminum precursors with dimethyl amide ligands, hydride ligands, and a novel reduced ${ }^{\mathrm{MeNacNac}}$ ligand. As with previous work, all compounds contained metal-nitrogen bonds and eliminated metal-carbon bonds to reduce the chance of incorporating carbon into AlN films during an ALD process. $\mathrm{Al}\left({ }^{\mathrm{MeNacNac}}\right)\left(\mathrm{NMe}_{2}\right)_{2}$ is a novel compound and was found to be thermally stable and volatile with a 1 Torr temperature of $74{ }^{\circ} \mathrm{C}$. When exposed to atmosphere, it produced an oxo-bridged structure with the elimination of dimethylamine. This is an important observation as it indicates that the large and stabilizing ${ }^{\mathrm{MeN}} \mathrm{NacNac}$ ligand protected the $\mathrm{Al}$ centre while one of the anchoring $\mathrm{NMe}_{2}$ ligands was eliminated. When $\mathrm{Al}\left({ }^{\mathrm{Me}} \mathrm{NacNac}\right) \mathrm{H}_{2}$ was thermally evaluated, it was found to decompose upon heating in an NMR boilup study. The elimination of $\mathrm{H}_{2}(\mathrm{~g})$ was observed and this compound was deemed not appropriate for use in

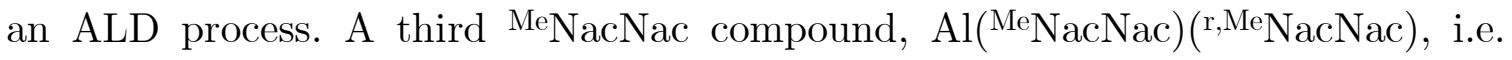
$\mathrm{Al}\left({ }^{\mathrm{MeNaCNac}}\right)\left(\left(\mathrm{NCH}_{3}\right) \mathrm{CH}\left(\mathrm{CH}_{3}\right) \mathrm{CHC}\left(\mathrm{CH}_{3}\right)\left(\mathrm{NCH}_{3}\right)\right)$ was also synthesized that is the first report of a reduced ${ }^{\mathrm{MeNacNac}}$ ligand. Its crystal structure was collected, 
and it had a calculated 1 Torr temperature of $131{ }^{\circ} \mathrm{C}$. This is the first report of this novel ligand, which could be isolated and employed on other metal centres. Future work should attempt to isolate this ligand as a protonated species so that it could be added over metal alkyl species, or lithiated to react with a variety of metal halides.

Finally, this work culminates in the synthesis of a heteroleptic aluminum hydride/amide precursor, $\mathrm{AlH}_{2}\left(\mathrm{NMe}_{2}\right)$, which was used to deposit pure AlN films by ALD in chapter 6. This precursor employed the aluminum-nitrogen bonds that have been discussed throughout this thesis in order to reduce carbon contamination in AlN films, but also added a hydride ligand compared to the initial work on $\mathrm{Al}\left(\mathrm{NMe}_{2}\right)_{3}$ presented in chapter 3 . The hydride ligand was designed into this precursor in an attempt to reduce oxygen impurities in AlN films. This design is important to consider during precursor development due to the fact that aluminum is an extremely oxophilic element; it is smart precursor design to combat a known issue that can occur during film growth in order to improve the quality of deposited materials. In this case, it was the intent that adding hydride ligands could introduce a reducing atmosphere during film growth that would resist film oxidation, and the $\mathrm{AlH}_{2}\left(\mathrm{NMe}_{2}\right)$ precursor with ammonia plasma was ultimately used to deposit AlN by ALD. Carbon and oxygen impurities were measured by XPS at $<1 \%$ and $<2 \%$, respectively, which can be attributed to this precursor design in removing metal- 
carbon bonds, implementing metal-nitrogen bonds, and using the hydride ligand to prevent AlN oxidation during growth. The AlN films grown were also found to be crystalline when grown at temperatures of $200{ }^{\circ} \mathrm{C}$ and above and were stoichiometric with an Al:N ratio of 1.07. The GPC using $\mathrm{AlH}_{2}\left(\mathrm{NMe}_{2}\right)$ and $\mathrm{NH}_{3}$ plasma to deposit AlN was approximately $4 \AA$, which is nearly four times the GPC reported for TMA processes, but still lies within lattice parameters of hexagonal AlN. This $\mathrm{AlH}_{2}\left(\mathrm{NMe}_{2}\right)$ precursor is extremely easy and cheap to synthesize, and compared to using TMA, can reduce materials cost for an AlN ALD process by up to two orders of magnitude. This work is limited to the deposition of AlN, but studies depositing $\mathrm{Al}_{2} \mathrm{O}_{3}$ using this precursor are in progress and could demonstrate this precursor's potential to replace TMA in alumina depositions by ALD.

The work presented in this thesis has explored a multitude of avenues for improving group 13 metal nitride vapour deposition processes. Several novel precursors have been synthesized and evaluated for ALD processes, and as a result, high quality films of group 13 metal nitrides have been produced. The impact of this work, however, is not limited to the production of high-quality group 13 nitride films, though it is a desired outcome since the reduction of impurities is crucial for their use in microelectronics. The impact of these studies spreads further to the general field of ALD precursor design, emphasizing its importance with respect to its influence on the quality of resultant deposited films. It is fundamentally 
interesting to synthesize and characterize any variety of novel precursors, but it is unlikely that many will be used outside of one's own research. If an ALD precursor is to be used in other laboratories or even in industry, its merits must outweigh the current industry standards in terms of cost, ease of synthesis or availability, and ultimately in quality of deposited films. It is paramount that future ALD precursors employ smart design to maximize all potential benefits (and minimize undesired aspects) of deposited thin films. Controlling precursor design is an avenue that should be exploited to control ALD chemistry and use to our advance to ultimately produce high quality films. 


\section{References}

(1) Belyanin, A. F.; Bouilov, L. L.; Zhirnov, V. V.; Kamenev, A. I.; Kovalskij, K. A.; Spitsyn, B. V. Application of Aluminum Nitride Films for Electronic Devices. Diam. Relat. Mater. 1999, 8 (2-5), 369-372.

https://doi.org/10.1016/s0925-9635(98)00412-9.

(2) Tian, L.; Ponton, S.; Benz, M.; Crisci, A.; Reboud, R.; Giusti, G.; Volpi, F.; Rapenne, L.; Vallée, C.; Pons, M.; et al. Aluminum Nitride Thin Films Deposited by Hydrogen Plasma Enhanced and Thermal Atomic Layer Deposition. Surf. Coatings Technol. 2018.

https://doi.org/10.1016/j.surfcoat.2018.04.031.

(3) Earnshaw, a.; Greenwood, N. Aluminium, Gallium, Indium and Thallium. Chem. Elem. 1997, 216-267. https://doi.org/10.1016/B978-0-7506-33659.50013-4.

(4) Shih, H.-Y.; Lin, M.-C.; Chen, L.-Y.; Chen, M.-J. Uniform GaN Thin Films Grown on (100) Silicon by Remote Plasma Atomic Layer Deposition. Nanotechnology 2015, 26 (1), 014002. https://doi.org/10.1088/0957$4484 / 26 / 1 / 014002$.

(5) Acharya, A. R.; Thoms, B. D.; Nepal, N.; Eddy, C. R. Surface Structure and Surface Kinetics of InN Grown by Plasma-Assisted Atomic Layer Epitaxy: A HREELS Study. J. Vac. Sci. Technol. A Vacuum, Surfaces, Film. 2015, 33 (2), 021401. https://doi.org/10.1116/1.4901873.

(6) Ruffenach, S.; Moret, M.; Briot, O.; Gil, B. Recent Advances in the MOVPE Growth of Indium Nitride. Phys. status solidi 2010, 207 (1), 9-18. https://doi.org/10.1002/pssa.200982642.

(7) Asif Khan, M.; Bhattarai, A.; Kuznia, J. N.; Olson, D. T. High Electron Mobility Transistor Based on a GaN-AlxGa 1-XN Heterojunction. Appl. Phys. Lett. 1993, 63 (9), 1214-1215. https://doi.org/10.1063/1.109775.

(8) Liu, X.-Y.; Zhao, S.-X.; Zhang, L.-Q.; Huang, H.-F.; Shi, J.-S.; Zhang, C.M.; Lu, H.-L.; Wang, P.-F.; Zhang, D. W. AlGaN/GaN MISHEMTs with 
AlN Gate Dielectric Grown by Thermal ALD Technique. Nanoscale Res. Lett. 2015, 10 (1), 109. https://doi.org/10.1186/s11671-015-0802-x.

(9) Moore, G. E. Cramming More Components onto Integrated Circuits. Electronics 1965, 38 (8), 114.

https://doi.org/10.1109/JPROC.1998.658762.

(10) Gates, B. D.; Xu, Q.; Stewart, M.; Ryan, D.; Willson, C. G.; Whitesides, G. M. New Approaches to Nanofabrication: Molding, Printing, and Other Techniques. Chemical Reviews. American Chemical Society April 2005, pp 1171-1196. https://doi.org/10.1021/cr030076o.

(11) Martin, P. M. Introduction to Surface Engineering and Functionally Engineered Materials; John Wiley and Sons: Hoboken, NJ, USA, 2011. https://doi.org/10.1002/9781118171899.

(12) Abegunde, O. O.; Akinlabi, E. T.; Oladijo, O. P.; Akinlabi, S.; Ude, A. U. Overview of Thin Film Deposition Techniques. 2019.

https://doi.org/10.3934/matersci.2019.2.174.

(13) Bryant, W. A. Review The Fundamentals Deposition of Chemical Vapour; 1977; Vol. 12.

(14) Uchida, Y.; Iwaizako, T.; Mizuno, S.; Tsuji, M.; Ago, H. Epitaxial Chemical Vapour Deposition Growth of Monolayer Hexagonal Boron Nitride on a $\mathrm{Cu}(111) /$ Sapphire Substrate. Phys. Chem. Chem. Phys. 2017, 19 (12), 8230-8235. https://doi.org/10.1039/c6cp08903h.

(15) Kern, W.; Vossen, J. L. Thin Film Processes II; Elsevier Inc., 2012. https://doi.org/10.1016/C2009-0-22311-7.

(16) Kim, H.; Oh, I.-K. Review of Plasma-Enhanced Atomic Layer Deposition: Technical Enabler of Nanoscale Device Fabrication. Jpn. J. Appl. Phys. 2014, 53, 1-7. https://doi.org/10.7567/JJAP.53.03DA01.

(17) Kim, H. Characteristics and Applications of Plasma Enhanced-Atomic Layer Deposition. In Thin Solid Films; Elsevier, 2011; Vol. 519, pp 66396644. https://doi.org/10.1016/j.tsf.2011.01.404.

(18) Kim, H.; Cabral, C.; Lavoie, C.; Rossnagel, S. M. Diffusion Barrier Properties of Transition Metal Thin Films Grown by Plasma-Enhanced Atomic-Layer Deposition. In Journal of Vacuum Science and Technology B: Microelectronics and Nanometer Structures; American Vacuum SocietyAVS, 2002; Vol. 20, pp 1321-1326. https://doi.org/10.1116/1.1486233. 
(19) Profijt, H. B.; Potts, S. E.; Van De Sanden, M. C. M.; Kessels, W. M. M. Plasma-Assisted Atomic Layer Deposition: Basics, Opportunities, and Challenges. J. Vac. Sci. Technol. A 2011, 29, 50801. https://doi.org/10.1116/1.3609974.

(20) Kunte, G. V; Shivashankar, S. A.; Umarji, A. M. Thermogravimetric Evaluation of the Suitability of Precursors for MOCVD. Meas. Sci. Technol. 2008, 19 (2), 025704. https://doi.org/10.1088/0957$0233 / 19 / 2 / 025704$.

(21) Land, M. A.; Robertson, K. N.; Barry, S. T. Ligand-Assisted Volatilization and Thermal Stability of Bis(Imido)Dichloromolybdenum(VI) ([(t$\mathrm{BuN}=) 2 \mathrm{MoCl} 2] 2$ ) and Its Adducts. Organometallics 2020, 39 (7), 916-927. https://doi.org/10.1021/acs.organomet.9b00578.

(22) Puurunen, R. L. Surface Chemistry of Atomic Layer Deposition: A Case Study for the Trimethylaluminum/Water Process. J. Appl. Phys. 2005, 97 (12), 121301. https://doi.org/10.1063/1.1940727.

(23) Schwille, M. C.; Schössler, T.; Barth, J.; Knaut, M.; Schön, F.; Höchst, A.; Oettel, M.; Bartha, J. W. Experimental and Simulation Approach for Process Optimization of Atomic Layer Deposited Thin Films in High Aspect Ratio 3D Structures. J. Vac. Sci. Technol. A Vacuum, Surfaces, Film. 2017, 35 (1), 01B118. https://doi.org/10.1116/1.4971196.

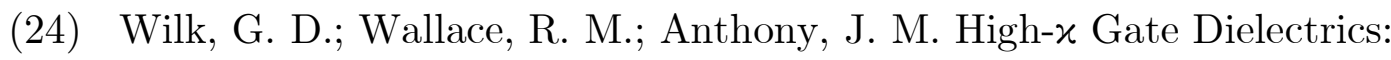
Current Status and Materials Properties Considerations. J. Appl. Phys. 2001, 89 (10), 5243-5275. https://doi.org/10.1063/1.1361065.

(25) Miikkulainen, V.; Leskelä, M.; Ritala, M.; Puurunen, R. L. Crystallinity of Inorganic Films Grown by Atomic Layer Deposition: Overview and General Trends. Journal of Applied Physics. American Institute of PhysicsAIP January 14, 2013, p 021301. https://doi.org/10.1063/1.4757907.

(26) Ezhovskii, Y. K.; Klusevich, A. I. Fabrication and Dielectric Properties of Multilayer Ta2O 5/Al2O3 Nanostructures. Inorg. Mater. 2003, 39 (10), 1062-1066. https://doi.org/10.1023/A:1026043209924.

(27) Koltsov, S. I.; Kopylov, V. B.; Smirnov, V. M.; Aleskovskii, V. B. Synthesis and Investigation of Aluminum-Oxygen Layers on Surface of Silica. $J$. Appl. Chem. USSR 1976, 49 (3), 525-528.

(28) Koltsov, S. I.; Smirnov, V. M.; Rachkovskii, R. R.; Malalaeva, T. V.; Aleskovskii, V. B. Study of Aluminosilicate Systems Synthesized by the Molecular-Layering Method. J. Appl. Chem. USSR 1978, 51 (11), 2475- 
2477.

(29) Heyman, A.; Musgrave, C. B. A Quantum Chemical Study of the Atomic Layer Deposition of Al $2 \mathrm{O} 3 \mathrm{Using} \mathrm{AlCl} 3$ and $\mathrm{H} 2 \mathrm{O}$ as Precursors. J.

Phys. Chem. B 2004, 108 (18), 5718-5725.

https://doi.org/10.1021/jp049762x.

(30) Räisänen, P. I.; Ritala, M.; Leskelä, M. Atomic Layer Deposition of Al2O3 Films Using $\mathrm{AlCl} 3$ and $\mathrm{Al}(\mathrm{OiPr}) 3$ as Precursors. J. Mater. Chem. 2002, 12 (5), 1415-1418. https://doi.org/10.1039/b201385c.

(31) Aarik, J.; Aidla, A.; Jaek, A.; Kiisler, A.-A.; Tammik, A.-A. Properties of Amorphous Al2O3 Films Grown by ALE. Acta Polytech. Scand. 1990, 195, 201-208.

(32) Oya, G. I.; Yoshida, M.; Sawada, Y. Growth of $\alpha$-Al2O3 Films by Molecular Layer Epitaxy. Appl. Phys. Lett. 1987, 51 (15), 1143-1145. https://doi.org/10.1063/1.98765.

(33) Oya, G. ichiro; Sawada, Y. Molecular Layer Epitaxy of $\alpha$-Al2O3 Films. J. Cryst. Growth 1990, 99 (1-4), 572-576. https://doi.org/10.1016/00220248(90)90585-9.

(34) Chen, Z.; Zhu, Z.; Härkönen, K.; Salmi, E. Batch Processing of Aluminum Nitride by Atomic Layer Deposition from $\mathrm{AlCl} 3$ and $\mathrm{NH} 3$. J. Vac. Sci. Technol. A 2019, 37 (2), 020925. https://doi.org/10.1116/1.5079509.

(35) Elers, K.-E.; Ritala, M.; Leskelä, M.; Johansson, L.-S. Atomic Layer Epitaxy Growth of AIN Thin Films. Le J. Phys. IV 1995, 05 (C5), C51021-C5-1027. https://doi.org/10.1051/jphyscol:19955120.

(36) Lee, Y. J.; Kang, S.-W. Growth of Aluminum Nitride Thin Films Prepared by Plasma-Enhanced Atomic Layer Deposition. Thin Solid Films 2004, 446 (2), 227-231. https://doi.org/10.1016/j.tsf.2003.10.004.

(37) Jokinen, J.; Haussalo, P.; Keinonen, J.; Ritala, M.; Riihelä, D.; Leskelä, M. Analysis of AlN Thin Films by Combining TOF-ERDA and NRB Techniques. Thin Solid Films 1996, 289 (1-2), 159-165. https://doi.org/10.1016/S0040-6090(96)08927-4.

(38) Lim, J. W.; Yun, J. S. Electrical Properties of Alumina Films by PlasmaEnhanced Atomic Layer Deposition. Electrochem. Solid-State Lett. 2004, 7 (8), F45. https://doi.org/10.1149/1.1756541.

(39) Niskanen, A.; Arstila, K.; Ritala, M.; Leskelä, M. Low-Temperature Deposition of Aluminum Oxide by Radical Enhanced Atomic Layer 
Deposition. J. Electrochem. Soc. 2005, 152 (7), F90.

https://doi.org/10.1149/1.1931471.

(40) Heil, S. B. S.; Kudlacek, P.; Langereis, E.; Engeln, R.; Van De Sanden, M. C. M.; Kessels, W. M. M. In Situ Reaction Mechanism Studies of PlasmaAssisted Atomic Layer Deposition of Al2O3. Appl. Phys. Lett. 2006, 89 (13), 131505. https://doi.org/10.1063/1.2357886.

(41) Kim, J. B.; Kwon, D. R.; Chakrabarti, K.; Lee, C.; Oh, K. Y.; Lee, J. H. Improvement in Al2O3 Dielectric Behavior by Using Ozone as an Oxidant for the Atomic Layer Deposition Technique. J. Appl. Phys. 2002, 92 (11), 6739-6742. https://doi.org/10.1063/1.1515951.

(42) Kim, S. K.; Lee, S. W.; Hwang, C. S.; Min, Y.-S.; Won, J. Y.; Jeong, J. Low Temperature $\left(<100^{\circ} \mathrm{C}\right)$ Deposition of Aluminum Oxide Thin Films by ALD with O[Sub 3] as Oxidant. J. Electrochem. Soc. 2006, 153 (5), F69. https://doi.org/10.1149/1.2177047.

(43) Rai, V. R.; Vandalon, V.; Agarwal, S. Surface Reaction Mechanisms during Ozone and Oxygen Plasma Assisted Atomic Layer Deposition of Aluminum Oxide. Langmuir 2010, 26 (17), 13732-13735.

https://doi.org/10.1021/la101485a.

(44) Ott, A. W.; Klaus, J. W.; Johnson, J. M.; George, S. M. Al3O3 Thin Film Growth on $\mathrm{Si}(100)$ Using Binary Reaction Sequence Chemistry. Thin Solid Films 1997, 292 (1-2), 135-144. https://doi.org/10.1016/S00406090(96)08934-1.

(45) Bergna, H. E. Colloid Chemistry of Silica; 1994; pp 1-47. https://doi.org/10.1021/ba-1994-0234.ch001.

(46) Puurunen, R. L.; Lindblad, M.; Rootc, A.; Krausea, A. O. I. Successive Reactions of Gaseous Trimethylaluminium and Ammonia on Porous Alumina. Phys. Chem. Chem. Phys. 2001, 3 (6), 1093-1102. https://doi.org/10.1039/b007249o.

(47) Riihelä, D.; Ritala, M.; Matero, R.; Leskelä, M.; Jokinen, J.; Haussalo, P. Low Temperature Deposition of AIN Films by an Alternate Supply of Trimethyl Aluminum and Ammonia. Chem. Vap. Depos. 1996, 2 (6), 277283. https://doi.org/10.1002/cvde.19960020612.

(48) Liu, H.; Bertolet, D. C.; Rogers, J. W. Reactions of Trimethylaluminum and Ammonia on Alumina at $600 \mathrm{~K}$ - Surface Chemical Aspects of AlN Thin Film Growth. Surf. Sci. 1995, 340 (1-2), 88-100. https://doi.org/10.1016/0039-6028(95)00598-6. 
(49) Kim, K.-H.; Kwak, N.-W.; Lee, S. H. Fabrication and Properties of AlN Film on GaN Substrate by Using Remote Plasma Atomic Layer Deposition Method. Electron. Mater. Lett. 2009, 5 (2), 83-86.

https://doi.org/10.3365/eml.2009.06.083.

(50) Alevli, M.; Ozgit, C.; Donmez, I.; Biyikli, N. The Influence of N 2 /H 2 and Ammonia N Source Materials on Optical and Structural Properties of AlN Films Grown by Plasma Enhanced Atomic Layer Deposition. 2011. https://doi.org/10.1016/j.jcrysgro.2011.09.003.

(51) Bosund, M.; Sajavaara, T.; Laitinen, M.; Huhtio, T.; Putkonen, M.; Airaksinen, V.-M.; Lipsanen, H. Properties of AlN Grown by Plasma Enhanced Atomic Layer Deposition. Appl. Surf. Sci. 2011, 257, 7827-7830. https://doi.org/10.1016/j.apsusc.2011.04.037.

(52) Goerke, S.; Ziegler, M.; Ihring, A.; Dellith, J.; Undisz, A.; Diegel, M.; Anders, S.; Huebner, U.; Rettenmayr, M.; Meyer, H.-G. Atomic Layer Deposition of AlN for Thin Membranes Using Trimethylaluminum and H 2 /N 2 Plasma. Appl. Surf. Sci. 2015, 338, 35-41. https://doi.org/10.1016/j.apsusc.2015.02.119.

(53) Slack, G. A.; McNelly, T. F. Growth of High Purity AlN Crystals. J. Cryst. Growth 1976, 34 (2), 263-279. https://doi.org/10.1016/00220248(76)90139-1.

(54) Biyikli, N.; Haider, A.; Altuntas, H.; Bayrak, T.; Kizir, S. Electrical Conduction and Dielectric Relaxation Properties of AlN Thin Films Grown by Hollow-Cathode Plasma-Assisted Atomic Layer Deposition. 2016. https://doi.org/10.1088/0268-1242/31/7/075003.

(55) Barry, S. T. Amidinates, Guanidinates and Iminopyrrolidinates: Understanding Precursor Thermolysis to Design a Better Ligand. Coord. Chem. Rev. 2013, 257 (23), 3192-3201. https://doi.org/10.1016/j.ccr.2013.03.015.

(56) Rowley, C. N.; DiLabio, G. A.; Barry, S. T. Theoretical and Synthetic Investigations of Carbodiimide Insertions into Al-CH3 and Al-N(CH3)2 Bonds. Inorg. Chem. 2005, 44 (6), 1983-1991. https://doi.org/10.1021/ic048501z.

(57) Brazeau, A. L.; Wang, Z.; Rowley, C. N.; Barry, S. T. Synthesis and Thermolysis of Aluminum Amidinates: A Ligand-Exchange Route for New Mixed-Ligand Systems. Inorg. Chem. 2006, 45 (5), 2276-2281. https://doi.org/10.1021/ic051856d. 
(58) Brazeau, A. L.; Barry, S. T. Atomic Layer Deposition of Aluminum Oxide Thin Films from a Heteroleptic, Amidinate-Containing Precursor. Chem. Mater. 2008, 20 (23), 7287-7291. https://doi.org/10.1021/cm802195b.

(59) Pallister, P. J.; Buttera, S. C.; Seán, S.; Barry, T. Quantitative Surface Coverage Calculations via Solid-State NMR for Thin Film Depositions: A Case Study for Silica and a Gallium Amidinate. J. Phys. Chem. C 2014, 118, 26. https://doi.org/10.1021/jp4102674.

(60) Pallister, P. J.; Buttera, S. C.; Barry, S. T. Self-seeding Gallium Oxide Nanowire Growth by Pulsed Chemical Vapor Deposition. Phys. status solidi 2015, 212 (7), 1514-1518. https://doi.org/10.1002/PSSA.201532275.

(61) Rowley, C. N.; DiLabio, G. A.; Barry, S. T. Theoretical and Synthetic Investigations of Carbodiimide Insertions into Al-CH3 and Al-N(CH3)2 Bonds. Inorg. Chem. 2005, 44 (6), 1983-1991.

https://doi.org/10.1021/ic048501z.

(62) Brazeau, A. L.; DiLabio, G. A.; Kreisel, K. A.; Monillas, W.; Yap, G. P. A.; Barry, S. T. Theoretical and Experimental Investigations of Ligand Exchange in Guanidinate Ligand Systems for Group 13 Metals. Dalt. Trans. 2007, No. 30, 3297. https://doi.org/10.1039/b706044k.

(63) Coyle, J. P.; Monillas, W. H.; Yap, G. P. A.; Barry, S. T. Synthesis and Thermal Chemistry of Copper (I) Guanidinates. Inorg. Chem. 2008, 47 (2), 683-689. https://doi.org/10.1021/ic701317y.

(64) Coyle, J. P.; Johnson, P. A.; Dilabio, G. A.; Barry, S. T.; Müller, J. GasPhase Thermolysis of a Guanidinate Precursor of Copper Studied by Matrix Isolation, Time-of-Flight Mass Spectrometry, and Computational Chemistry. Inorg. Chem. 2010, 49 (6), 2844-2850. https://doi.org/10.1021/ic902247w.

(65) Weidler, N.; Paulus, S.; Schuch, J.; Klett, J.; Hoch, S.; Stenner, P.; Maljusch, A.; Brötz, J.; Wittich, C.; Kaiser, B.; et al. CoOx Thin Film Deposited by CVD as Efficient Water Oxidation Catalyst: Change of Oxidation State in XPS and Its Correlation to Electrochemical Activity. Phys. Chem. Chem. Phys. 2016, 18 (16), 10708-10718.

https://doi.org/10.1039/c5cp05691h.

(66) Junge Puring, K.; Zywitzki, D.; Taffa, D. H.; Rogalla, D.; Winter, M.; Wark, M.; Devi, A. Rational Development of Cobalt $\beta$-Ketoiminate Complexes: Alternative Precursors for Vapor-Phase Deposition of Spinel Cobalt Oxide Photoelectrodes. Inorg. Chem. 2018, 57 (9), 5133-5144. https://doi.org/10.1021/acs.inorgchem.8b00204. 
(67) Liu, H.; Rogers, J. W. Molecularly Engineered Low Temperature Atomic Layer Growth of Aluminum Nitride on Si(100). J. Vac. Sci. Technol. A Vacuum, Surfaces, Film. 1999, 17 (2), 325-331.

https://doi.org/10.1116/1.581591.

(68) Blakeney, K. J.; Winter, C. H. Atomic Layer Deposition of Aluminum Metal Films Using a Thermally Stable Aluminum Hydride Reducing Agent. https://doi.org/10.1021/acs.chemmater.8b00445.

(69) Bhuiyan, A. G.; Hashimoto, A.; Yamamoto, A. Indium Nitride (InN): A Review on Growth, Characterization, and Properties. Journal of Applied Physics. American Institute of PhysicsAIP September 1, 2003, pp 27792808. https://doi.org/10.1063/1.1595135.

(70) Bellotti, E.; Doshi, B. K.; Brennan, K. F.; Albrecht, J. D.; Ruden, P. P. Ensemble Monte Carlo Study of Electron Transport in Wurtzite InN. J. Appl. Phys. 1999, 85 (2), 916-923. https://doi.org/10.1063/1.369211.

(71) Butcher, K. S. A.; Tansley, T. L. InN, Latest Development and a Review of the Band-Gap Controversy. Superlattices Microstruct. 2005, 38 (1), 137. https://doi.org/10.1016/j.spmi.2005.03.004.

(72) Ivanov, S. V.; Shubina, T. V.; Komissarova, T. A.; Jmerik, V. N. Metastable Nature of InN and In-Rich InGaN Alloys. J. Cryst. Growth 2014, 403, 83-89. https://doi.org/10.1016/j.jcrysgro.2014.06.019.

(73) Nepal, N.; Mahadik, N. A.; Nyakiti, L. O.; Qadri, S. B.; Mehl, M. J.; Hite, J. K.; Eddy, C. R. Epitaxial Growth of Cubic and Hexagonal InN Thin Films via Plasma-Assisted Atomic Layer Epitaxy. Cryst. Growth Des. 2013, 13 (4), 1485-1490. https://doi.org/10.1021/cg3016172.

(74) Haider, A.; Kizir, S.; Biyikli, N. Low-Temperature Self-Limiting Atomic Layer Deposition of Wurtzite $\mathrm{InN}$ on $\mathrm{Si}(100)$. AIP Adv. 2016, 6 (4), 045203. https://doi.org/10.1063/1.4946786.

(75) Buttera, S. C.; Mandia, D. J.; Barry, S. T. Tris(Dimethylamido)Aluminum(III): An Overlooked Atomic Layer Deposition Precursor. J. Vac. Sci. Technol. A Vacuum, Surfaces, Film. 2017, 35 (1), 01B128. https://doi.org/10.1116/1.4972469.

(76) Lide, D. R.; Baysinger, G.; Berger, L. I.; Goldberg, R. N.; Kehiaian, H. V; Kuchitsu, K.; Roth, D. L.; Zwillinger, D. CRC Handbook of Chemistry and Physics.

(77) Saitoh, H.; Utsumi, W.; Aoki, K. Decomposition of InN at High Pressures and Temperatures and Its Thermal Instability at Ambient Conditions. $J$. 
Cryst. Growth 2008, 310 (2), 473-476.

https://doi.org/10.1016/j.jcrysgro.2007.10.037.

(78) Barry, S. T.; Gordon, P. G.; Ward, M. J.; Heikkila, M. J.; Monillas, W. H.; Yap, G. P. A.; Ritala, M.; Leskelä, M. Chemical Vapour Deposition of In2O3 Thin Films from a Tris-Guanidinate Indium Precursor. Dalton Trans. 2011, 40 (37), 9425-9430. https://doi.org/10.1039/c1dt10877h.

(79) Gebhard, M.; Hellwig, M.; Kroll, A.; Rogalla, D.; Winter, M.; Mallick, B.;

Ludwig, A.; Wiesing, M.; Wieck, A. D.; Grundmeier, G.; et al. New Amidinate Complexes of Indium(III): Promising CVD Precursors for Transparent and Conductive In2O3 Thin Films. Dalt. Trans. 2017, 46 (31), 10220-10231. https://doi.org/10.1039/c7dt01280b.

(80) Gebhard, M.; Hellwig, M.; Parala, H.; Xu, K.; Winter, M.; Devi, A. Indium-Tris-Guanidinates: A Promising Class of Precursors for Water Assisted Atomic Layer Deposition of In2O3 Thin Films. Dalton Trans. 2014, 43 (3), 937-940. https://doi.org/10.1039/c3dt52746h.

(81) Ziffle, L. C.; Kenney, A. P.; Barry, S. T.; Müller, J. Thermal Fragmentation of the Guanidinato Aluminum Amide Precursor [Me2NC(NiPr)2]Al(NMe2)2: An Investigation of Reactive Species by Matrix-Isolation FTIR Spectroscopy and Time-of-Flight Mass Spectrometry. Polyhedron 2008, 27 (6), 1832-1840. https://doi.org/10.1016/j.poly.2008.02.024.

(82) Frisch, M. J.; Trucks, G. W.; Schlegel, H. B.; Scuseria, G. E.; Robb, M. A.; Cheeseman, J. R.; Scalmani, G.; Barone, V.; Petersson, G. A.; Nakatsuji, H.; et al. Gaussian 09, Revision E.01. Gaussian, Inc. 2013.

(83) Becke, A. D. Density-Functional Thermochemistry. III. The Role of Exact Exchange. J. Chem. Phys. 1993, 98 (7), 5648-5652.

https://doi.org/10.1063/1.464913.

(84) Lee, C.; Yang, W.; Parr, R. G. Development of the Colle-Salvetti Correlation-Energy Formula into a Functional of the Electron Density. Phys. Rev. B 1988, 37 (2), 785-789.

https://doi.org/10.1103/PhysRevB.37.785.

(85) Bauschlicher, C. W. Accurate Indium Bond Energies. J. Phys. Chem. A 1999, 103 (32), 6429-6432. https://doi.org/10.1021/jp990967t.

(86) Kenney, A. P.; Yap, G. P. A.; Richeson, D. S.; Barry, S. T. The Insertion of Carbodiimides into $\mathrm{Al}$ and Ga Amido Linkages. Guanidinates and Mixed Amido Guanidinates of Aluminum and Gallium. Inorg. Chem. 2005, 44 
(8), 2926-2933. https://doi.org/10.1021/ic048433g.

(87) Wasslen, Y. A.; Kurek, A.; Johnson, P. A.; Pigeon, T. C.; Monillas, W. H.; Yap, G. P. A.; Barry, S. T. Heteroleptic Iminopyrrolidinates of Aluminium. Dalton Trans. 2010, 39 (38), 9046-9054.

https://doi.org/10.1039/c0dt00267d.

(88) Whitehorne, T. J. J.; Coyle, J. P.; Mahmood, A.; Monillas, W. H.; Yap, G. P. A.; Barry, S. T. Group 11 Amidinates and Guanidinates: Potential Precursors for Vapour Deposition. Eur. J. Inorg. Chem. 2011, 2011 (21), 3240-3247. https://doi.org/10.1002/ejic.201100262.

(89) James, A. M.; Laxman, R. K.; Fronczek, F. R.; Maverick, A. W. Phosphorescence and Structure of a Tetrameric Copper(I)-Amide Cluster. Inorg. Chem. 1998, 37 (15), 3785-3791. https://doi.org/10.1021/ic971341p.

(90) Li, Z.; Rahtu, A.; Gordon, R. G. Atomic Layer Deposition of Ultrathin Copper Metal Films from a Liquid Copper(I) Amidinate Precursor. 2006. https://doi.org/10.1149/1.2338632.

(91) Kim, K. H.; Gordon, R. G.; Ritenour, A.; Antoniadis, D. A. Atomic Layer Deposition of Insulating Nitride Interfacial Layers for Germanium Metal Oxide Semiconductor Field Effect Transistors with High-??

Oxide/Tungsten Nitride Gate Stacks. Appl. Phys. Lett. 2007, 90 (21), 212104. https://doi.org/10.1063/1.2741609.

(92) Piazza, G. Integrated Aluminum Nitride Piezoelectric Microelectromechanical System for Radio Front Ends. J. Vac. Sci. Technol. A Vacuum, Surfaces, Film. 2009, 27 (4), 776-784. https://doi.org/10.1116/1.3077276.

(93) Cordier, Y.; Frayssinet, E.; Chmielowska, M.; Nemoz, M.; Courville, A.; Vennéguès, P.; Mierry, P. De; Chenot, S.; Camus, J.; Aissa, K. A.; et al. GaN High Electron Mobility Transistors on Silicon Substrates with MBE/PVD AlN Seed Layers. Phys. status solidi 2014, 11 (3-4), 498-501. https://doi.org/10.1002/pssc.201300453.

(94) Waggoner, K. M.; Olmstead, M. M.; Power, P. P. Structural and Spectroscopic Characterization of the Compounds [Al(NMe2)3]2, $[\mathrm{Ga}(\mathrm{NMe} 2) 3] 2,[(\mathrm{Me} 2 \mathrm{~N}) 2 \mathrm{Al}\{\mu-\mathrm{N}(\mathrm{H}) 1-\mathrm{Ad}\}] 2(1-\mathrm{Ad}=1$-Adamantanyl) and $[\{\mathrm{Me}(\mu-\mathrm{NPh} 2) \mathrm{Al}\} 2 \mathrm{NPh}(\mu-\mathrm{C} 6 \mathrm{H} 4)]$. Polyhedron 1990, 9 (2-3), 257-263. https://doi.org/10.1016/S0277-5387(00)80578-1.

(95) Groner, M. D.; Fabreguette, F. H.; Elam, J. W.; George, S. M. LowTemperature Al2O3 Atomic Layer Deposition. Chem. Mater. 2004, 16 (4), 
639-645. https://doi.org/10.1021/cm0304546.

(96) Ylivaara, O. M. E.; Liu, X.; Kilpi, L.; Lyytinen, J.; Schneider, D.; Laitinen, M.; Julin, J.; Ali, S.; Sintonen, S.; Berdova, M.; et al. Aluminum Oxide from Trimethylaluminum and Water by Atomic Layer Deposition: The Temperature Dependence of Residual Stress, Elastic Modulus, Hardness and Adhesion. Thin Solid Films 2014, 552, 124-135.

https://doi.org/10.1016/j.tsf.2013.11.112.

(97) Broas, M.; Sippola, P.; Sajavaara, T.; Vuorinen, V.; Pyymaki Perros, A.; Lipsanen, H.; Paulasto-Kröckel, M. Structural and Chemical Analysis of Annealed Plasma-Enhanced Atomic Layer Deposition Aluminum Nitride Films. J. Vac. Sci. Technol. A Vacuum, Surfaces, Film. 2016, 34 (4), 041506. https://doi.org/10.1116/1.4953029.

(98) Goerke, S.; Ziegler, M.; Ihring, A.; Dellith, J.; Undisz, A.; Diegel, M.; Anders, S.; Huebner, U.; Rettenmayr, M.; Meyer, H.-G. Atomic Layer Deposition of AlN for Thin Membranes Using Trimethylaluminum and H2/N2 Plasma. Appl. Surf. Sci. 2015, 338, 35-41. https://doi.org/10.1016/j.apsusc.2015.02.119.

(99) Nepal, N.; Qadri, S. B.; Hite, J. K.; Mahadik, N. A.; Mastro, M. A.; Eddy, C. R. Epitaxial Growth of AlN Films via Plasma-Assisted Atomic Layer Epitaxy. Appl. Phys. Lett. 2013, 103 (8), 082110.

https://doi.org/10.1063/1.4818792.

(100) Van Bui, H.; Wiggers, F. B.; Gupta, A.; Nguyen, M. D.; Aarnink, A. A. I.; de Jong, M. P.; Kovalgin, A. Y. Initial Growth, Refractive Index, and Crystallinity of Thermal and Plasma-Enhanced Atomic Layer Deposition AlN Films. J. Vac. Sci. Technol. A Vacuum, Surfaces, Film. 2015, 33 (1), 01A111. https://doi.org/10.1116/1.4898434.

(101) Motamedi, P.; Cadien, K. XPS Analysis of AlN Thin Films Deposited by Plasma Enhanced Atomic Layer Deposition. Appl. Surf. Sci. 2014, 315 (1), 104-109. https://doi.org/10.1016/j.apsusc.2014.07.105.

(102) Motamedi, P.; Cadien, K. Structural and Optical Characterization of LowTemperature ALD Crystalline AlN. J. Cryst. Growth 2015, 421, 45-52. https://doi.org/10.1016/j.jcrysgro.2015.04.009.

(103) Crist, B. V. Handbooks of Monochromatic XPS Spectra Volume 1-The Elements and Native Oxides; 1999.

(104) Kim, O. H.; Kim, D.; Anderson, T. Atomic Layer Deposition of GaN Using GaCl3 and NH3. J. Vac. Sci. Technol. A Vacuum, Surfaces, Film. 2009, 
27 (4), 923-928. https://doi.org/10.1116/1.3106619.

(105) Strite, S.; Morkoç, H. GaN, AlN, and InN: A Review. J. Vac. Sci. Technol. B 1992, 10, 1237-1266.

(106) Porowski, S. Growth and Properties of Single Crystalline GaN Substrates and Homoepitaxial Layers. Mater. Sci. Eng. B 1997, 44 (1-3), 407-413. https://doi.org/10.1016/S0921-5107(96)01730-8.

(107) Lakshmi, E. Dielectric Properties of Reactively Sputtered Gallium Nitride Films. Thin Solid Films 1981, 83 (1), 137-140. https://doi.org/10.1016/0040-6090(81)90597-6.

(108) Suzuki, M.; Uenoyama, T.; Yanase, A. First-Principles Calculations of Effective Mass. Phys. Rev. B 1995, 52 (11).

(109) Burk, A. A.; O’Loughlin, M. J.; Siergiej, R. R.; Agarwal, A. K.; Sriram, S.; Clarke, R. C.; MacMillan, M. F.; Balakrishna, V.; Brandt, C. D. SiC and GaN Wide Bandgap Semiconductor Materials and Devices. Solid. State. Electron. 1999, 43 (8), 1459-1464. https://doi.org/10.1016/S00381101(99)00089-1.

(110) GaN-Based Materials and Devices; Shur, M. S., Davis, R. F., Eds.; World Scientific Publishing Co. Pte. Ltd.: Singapore, 2004.

(111) Tamura, K.; Kuroki, Y.; Yasui, K.; Suemitsu, M.; Ito, T.; Endou, T.; Nakazawa, H.; Narita, Y.; Takata, M.; Akahane, T. Growth of GaN on $\mathrm{SiC} / \mathrm{Si}$ Substrates Using AlN Buffer Layer by Hot-Mesh CVD. Thin Solid Films 2008, 516 (5), 659-662. https://doi.org/10.1016/j.tsf.2007.06.200.

(112) Lu, J.; Chen, J. T.; Dahlqvist, M.; Kabouche, R.; Medjdoub, F.; Rosen, J.; Kordina, O.; Hultman, L. Transmorphic Epitaxial Growth of AlN Nucleation Layers on SiC Substrates for High-Breakdown Thin GaN Transistors. Appl. Phys. Lett. 2019, 115 (22). https://doi.org/10.1063/1.5123374.

(113) Chen, J.-T.; Bergsten, J.; Lu, J.; Janzén, E.; Thorsell, M.; Hultman, L.; Rorsman, N.; Kordina, O. A GaN-SiC Hybrid Material for High-Frequency and Power Electronics. Appl. Phys. Lett. 2018, 113 (4), 41605. https://doi.org/10.1063/1.5042049.

(114) Liu, B. L.; Lachab, M.; Jia, A.; Yoshikawaa, A.; Takahashi, K. MOCVD Growth of Device-Quality GaN on Sapphire Using a Three-Step Approach. J. Cryst. Growth 2002, 234 (4), 637-645. https://doi.org/10.1016/S00220248(01)01755-9. 
(115) Ozgit-Akgun, C.; Goldenberg, E.; Okyay, A. K.; Biyikil, N. Hollow Cathode Plasma-Assisted Atomic Layer Deposition of Crystalline AlN, GaN and AlxGa1-XN Thin Films at Low Temperatures. J. Mater. Chem. C 2014, 2, 2123-2136.

(116) Ozgit, C.; Donmez, I.; Alevli, M.; Biyikli, N. Atomic Layer Deposition of GaN at Low Temperatures. J. Vac. Sci. Technol. A Vacuum, Surfaces, Film. 2012, 30 (1), 01A124. https://doi.org/10.1116/1.3664102.

(117) Banerjee, S.; Aarnink, A. A. I.; Gravesteijn, D. J.; Kovalgin, A. Y. Thermal Atomic Layer Deposition of Polycrystalline Gallium Nitride. J. Phys. Chem. C 2019, 123 (37), 23214-23225. https://doi.org/10.1021/acs.jpcc.9b05946.

(118) Alevli, M.; Haider, A.; Kizir, S.; Leghari, S. A.; Biyikli, N. Comparison of Trimethylgallium and Triethylgallium as "Ga" Source Materials for the Growth of Ultrathin GaN Films on Si (100) Substrates via Hollow-Cathode Plasma-Assisted Atomic Layer Deposition. J. Vac. Sci. Technol. A Vacuum, Surfaces, Film. 2016, 34 (1), 01A137. https://doi.org/10.1116/1.4937725.

(119) Motamedi, P.; Dalili, N.; Cadien, K. A Route to Low Temperature Growth of Single Crystal GaN on Sapphire. J. Mater. Chem. C 2015, 3 (28), 74287436. https://doi.org/10.1039/c5tc01556a.

(120) Kumagai, Y.; Mayumi, M.; Koukitu, A.; Seki, H. In Situ Gravimetric Monitoring of Halogen Transport Atomic Layer Epitaxy of Cubic-GaN. Appl. Surf. Sci. 2000, 159, 427-431. https://doi.org/10.1016/S01694332(00)00120-3.

(121) Gordon, R. G. Atomic Layer Deposition for Semiconductors. In Atomic Layer Deposition for Semiconductors; Hwang, C. S., Yoo, C. Y., Eds.; Springer US: New York, 2014; pp 15-46.

(122) O’Donoghue, R.; Rechmann, J.; Aghaee, M.; Rogalla, D.; Becker, H. W.; Creatore, M.; Wieck, A. D.; Devi, A. Low Temperature Growth of Gallium Oxide Thin Films via Plasma Enhanced Atomic Layer Deposition. Dalt. Trans. 2017, 46 (47), 16551-16561. https://doi.org/10.1039/c7dt03427j.

(123) Dezelah IV, C. L.; Niinistö, J.; Arstila, K.; Niinistö, L.; Winter, C. H. Atomic Layer Deposition of Ga $2 \mathrm{O} 3$ Films from a Dialkylamido-Based Precursor. Chem. Mater. 2006, 18 (2), 471-475. https://doi.org/10.1021/cm0521424.

(124) Meng, X.; Libera, J. A.; Fister, T. T.; Zhou, H.; Hedlund, J. K.; Fenter, P.; 
Elam, J. W. Atomic Layer Deposition of Gallium Sulfide Films Using Hexakis(Dimethylamido)Digallium and Hydrogen Sulfide. Chem. Mater. 2014, 26 (2), 1029-1039. https://doi.org/10.1021/cm4031057.

(125) Hoffman, D. M.; Prakash Rangarajan, S.; Athavale, S. D.; Economou, D. J.; Liu, J.; Zheng, Z.; Chu, W. Chemical Vapor Deposition of Aluminum and Gallium Nitride Thin Films from Metalorganic Precursors. J. Vac. Sci. Technol. A Vacuum, Surfaces, Film. 1996, 14 (2), 306-311. https://doi.org/10.1116/1.579893.

(126) Waggoner, K. M.; Olmstead, M. M.; Power, P. P. Structural and Spectroscopic Characterization of the Compounds [Al(NMe2)3]2, $[\mathrm{Ga}(\mathrm{NMe} 2) 3] 2,[(\mathrm{Me} 2 \mathrm{~N}) 2 \mathrm{Al}\{\mu-\mathrm{N}(\mathrm{H}) 1-\mathrm{Ad}\}] 2(1-\mathrm{Ad}=1$-Adamantanyl $)$ and $[\{\mathrm{Me}(\mu-\mathrm{NPh} 2) \mathrm{Al}\} 2 \mathrm{NPh}(\mu-\mathrm{C} 6 \mathrm{H} 4)]$. Polyhedron 1990, 9 (2-3), 257-263. https://doi.org/10.1016/S0277-5387(00)80578-1.

(127) Kern, W.; Puotinen, A. D. Cleaning Solutions Based on Hydrogen Peroxide for Use in Silicon Semiconductor Technology. RCA Rev. 1970, 31 (2), 187206.

(128) Deminskyi, P.; Rouf, P.; Ivanov, I. G.; Pedersen, H. Atomic Layer Deposition of InN Using Trimethylindium and Ammonia Plasma. J. Vac. Sci. Technol. A 2019, 37 (2), 20926. https://doi.org/10.1116/1.5079279.

(129) Mayer, M.; Eckstein, W.; Langhuth, H.; Schiettekatte, F.; Von Toussaint, U. Computer Simulation of Ion Beam Analysis: Possibilities and Limitations. Nucl. Instruments Methods Phys. Res. Sect. B Beam Interact. with Mater. Atoms 2011, 269 (24), 3006-3013.

https://doi.org/10.1016/j.nimb.2011.04.066.

(130) Ström, P.; Petersson, P.; Rubel, M.; Possnert, G. A Combined Segmented Anode Gas Ionization Chamber and Time-of-Flight Detector for Heavy Ion Elastic Recoil Detection Analysis. Rev. Sci. Instrum. 2016, 87 (10). https://doi.org/10.1063/1.4963709.

(131) K.S. Janson. CONTES. Uppsala University, Sweden 2004.

(132) Arstila, K.; Julin, J.; Laitinen, M. I.; Aalto, J.; Konu, T.; Kärkkäinen, S.; Rahkonen, S.; Raunio, M.; Itkonen, J.; Santanen, J. P.; et al. Potku - New Analysis Software for Heavy Ion Elastic Recoil Detection Analysis. Nucl. Instruments Methods Phys. Res. Sect. B Beam Interact. with Mater. Atoms 2014, 331, 34-41. https://doi.org/10.1016/j.nimb.2014.02.016.

(133) Zhang, Y.; Whitlow, H. J.; Winzell, T.; Bubb, I. F.; Sajavaara, T.; Arstila, K.; Keinonen, J. Detection Efficiency of Time-of-Flight Energy Elastic 
Recoil Detection Analysis Systems. Nucl. Instruments Methods Phys. Res. Sect. B Beam Interact. with Mater. Atoms 1999, 149 (4), 477-489. https://doi.org/10.1016/S0168-583X(98)00963-X.

(134) Arvizu, M. A.; Wen, R. T.; Primetzhofer, D.; Klemberg-Sapieha, J. E.; Martinu, L.; Niklasson, G. A.; Granqvist, C. G. Galvanostatic Ion Detrapping Rejuvenates Oxide Thin Films. ACS Appl. Mater. Interfaces 2015, 7 (48), 26387-26390. https://doi.org/10.1021/acsami.5b09430.

(135) Qu, H. Y.; Primetzhofer, D.; Arvizu, M. A.; Qiu, Z.; Cindemir, U.; Granqvist, C. G.; Niklasson, G. A. Electrochemical Rejuvenation of Anodically Coloring Electrochromic Nickel Oxide Thin Films. ACS Appl. Mater. Interfaces 2017, 9 (49), 42420-42424. https://doi.org/10.1021/acsami.7b13815.

(136) Ziegler, J. F.; Ziegler, M. D.; Biersack, J. P. SRIM - The Stopping and Range of Ions in Matter (2010). Nucl. Instruments Methods Phys. Res. Sect. B Beam Interact. with Mater. Atoms 2010, 268 (11-12), 1818-1823. https://doi.org/10.1016/j.nimb.2010.02.091.

(137) Fujikane, M.; Leszczyński, M.; Nagao, S.; Nakayama, T.; Yamanaka, S.; Niihara, K.; Nowak, R. Elastic-Plastic Transition during Nanoindentation in Bulk GaN Crystal. J. Alloys Compd. 2008, 450 (1-2), 405-411. https://doi.org/10.1016/j.jallcom.2006.10.121.

(138) Moldovan, G.; Roe, M. J.; Harrison, I.; Kappers, M.; Humphreys, C. J.; Brown, P. D. Effects of KOH Etching on the Properties of Ga-Polar n-GaN Surfaces. Philos. Mag. 2006, 86 (16), 2315-2327. https://doi.org/10.1080/14786430500522628.

(139) Majlinger, Z.; Bozanic, A.; Petravic, M.; Kim, K. J.; Kim, B.; Yang, Y. W. NEXAFS and XPS Study of GaN Formation on Ion-Bombarded GaAs Surfaces. Vacuum 2009, 84 (1), 41-44. https://doi.org/10.1016/j.vacuum.2009.04.024.

(140) Kumar, M.; Kumar, A.; Thapa, S. B.; Christiansen, S.; Singh, R. XPS Study of Triangular GaN Nano/Micro-Needles Grown by MOCVD Technique. Mater. Sci. Eng. B Solid-State Mater. Adv. Technol. 2014, 186 (1), 89-93. https://doi.org/10.1016/j.mseb.2014.03.010.

(141) Kushvaha, S. S.; Kumar, M. S.; Maheshwari, M.; Shukla, A. K.; Pal, P.; Maurya, K. K. Structural and Electronic Properties of Epitaxial GaN Layer Grown on Sapphire (0001) Using Laser Molecular Beam Epitaxy. Mater. Res. Express 2014, 1 (3). https://doi.org/10.1088/2053-1591/1/3/035903. 
(142) Skuridina, D.; Dinh, V. D.; Lacroix, B.; Ruterana, P.; Hoffmann, M.; Sitar, Z.; Pristovsek, M.; Kneissl, M.; Vogt, P. Polarity Determination of Polar and Semipolar (1122) InN and GaN Layers by Valence Band Photoemission Spectroscopy. J. Appl. Phys. 2013, 114 (17). https:/ / doi.org/10.1063/1.4828487.

(143) Xu, X.; Liu, H.; Shi, C.; Zhao, Y.; Fung, S.; Beling, C. D. Residual Donors and Compensation in Metalorganic Chemical Vapor Deposition As-Grown n-GaN. J. Appl. Phys. 2001, 90 (12), 6130-6134.

https://doi.org/10.1063/1.1413706.

(144) Neugebauer, J.; de Walle, C. G. Gallium Vacancies and the Yellow Luminescence in GaN. Appl. Phys. Lett. 1996, 69 (4), 503-505. https://doi.org/10.1063/1.117767.

(145) Neugebauer, J.; Van De Walle, C. G. Atomic Geometry and Electronic Structure of Native Defects in GaN. Phys. Rev. B 1994, 50 (11), 80678070. https://doi.org/10.1103/PhysRevB.50.8067.

(146) Edwards, V. N.; Bremser, M. D.; Davis, R. F.; Batchelor, A. D.; Yoo, S. D.; Karan, C. F.; Aspnes, D. E. Trends in Residual Stress for GaN/AlN/6H-SiC Heterostructures. Appl. Phys. Lett. 1998, 73 (19), 28082810. https://doi.org/10.1063/1.122597.

(147) Davis, R. F. Organometallic Vapor Phase Epitaxial Growth of Group III Nitrides. In Comprehensive Semiconductor Science and Technology; Elsevier Inc., 2011; Vol. 1-6, pp 339-367. https://doi.org/10.1016/B978-044-453153-7.00100-0.

(148) Mihopoulos, T. G.; Gupta, V.; Jensen, K. F. A Reaction-Transport Model for AlGaN MOVPE Growth. J. Cryst. Growth 1998, 195 (1-4), 733-739. https://doi.org/10.1016/S0022-0248(98)00649-6.

(149) Ravasio, S.; Momose, T.; Fujii, K.; Shimogaki, Y.; Sugiyama, M.; Cavallotti, C. Analysis of the Gas Phase Kinetics Active during GaN Deposition from NH3 and Ga(CH3)3. J. Phys. Chem. A 2015, 119 (28), 7858-7871. https://doi.org/10.1021/acs.jpca.5b01425.

(150) Bergmann, U.; Reimer, V.; Atakan, B. An Experimental Study of the Reactions of Trimethylgallium with Ammonia and Water over a Wide Temperature Range. Phys. Chem. Chem. Phys. 1999, 1 (24), 5593-5599. https://doi.org/10.1039/a906452d.

(151) Sekiguchi, K.; Shirakawa, H.; Chokawa, K.; Araidai, M.; Kangawa, Y.; Kakimoto, K.; Shiraishi, K. Thermodynamic Analysis of Trimethylgallium 
Decomposition during GaN Metal Organic Vapor Phase Epitaxy. In Japanese Journal of Applied Physics; Japan Society of Applied Physics, 2018; Vol. 57, p 04FJ03. https://doi.org/10.7567/JJAP.57.04FJ03.

(152) Creighton, J. R.; Wang, G. T. Kinetics of Metal Organic-Ammonia Adduct Decomposition: Implications for Group-III Nitride MOCVD. J. Phys. Chem. A 2005, 109 (46), 10554-10562. https://doi.org/10.1021/jp054380s.

(153) An, Q.; Jaramillo-Botero, A.; Liu, W. G.; Goddard, W. A. Reaction Pathways of GaN (0001) Growth from Trimethylgallium and Ammonia versus Triethylgallium and Hydrazine Using First Principle Calculations. $J$. Phys. Chem. C 2015, 119 (8), 4095-4103.

https://doi.org/10.1021/jp5116405.

(154) Kakanakova-Georgieva, A.; Gueorguiev, G. K.; Stafström, S.; Hultman, L.; Janzén, E. AlGaInN Metal-Organic-Chemical-Vapor-Deposition Gas-Phase Chemistry in Hydrogen and Nitrogen Diluents: First-Principles Calculations. Chem. Phys. Lett. 2006, 431 (4-6), 346-351. https://doi.org/10.1016/j.cplett.2006.09.102.

(155) Nakamura, K.; Makino, O.; Tachibana, A.; Matsumoto, K. Quantum Chemical Study of Parasitic Reaction in III-V Nitride Semiconductor Crystal Growth. J. Organomet. Chem. 2000, 611 (1-2), 514-524. https://doi.org/10.1016/S0022-328X(00)00403-4.

(156) Sengupta, D.; Mazumder, S.; Kuykendall, W.; Lowry, S. A. Combined Ab Initio Quantum Chemistry and Computational Fluid Dynamics Calculations for Prediction of Gallium Nitride Growth. J. Cryst. Growth 2005, 279 (3-4), 369-382. https://doi.org/10.1016/j.jcrysgro.2005.02.036.

(157) Chen, C. H.; Liu, H.; Steigerwald, D.; Imler, W.; Kuo, C. P.; Craford, M. G.; Ludowise, M.; Lester, S.; Amano, J. A Study of Parasitic Reactions between NH3 and TMGa or TMAI. J. Electron. Mater. 1996, 25 (6), 1004-1008. https://doi.org/10.1007/BF02666736.

(158) Moscatelli, D.; Caccioppoli, P.; Cavallotti, C. Ab Initio Study of the Gas Phase Nucleation Mechanism of GaN. Appl. Phys. Lett. 2005, 86 (9), 1-3. https://doi.org/10.1063/1.1870129.

(159) Hirako, A.; Kusakabe, K.; Ohkawa, K. Modeling of Reaction Pathways of GaN Growth by Metalorganic Vapor-Phase Epitaxy Using TMGa/NH3/H2 System: A Computational Fluid Dynamics Simulation Study. Japanese J. Appl. Physics, Part 1 Regul. Pap. Short Notes Rev. Pap. 2005, 44 (2), 874-879. https://doi.org/10.1143/JJAP.44.874. 
(160) Beaumont, B.; Gibart, P.; Faurie, J. P. Nitrogen Precursors in Metalorganic Vapor Phase Epitaxy of (Al,Ga)N. J. Cryst. Growth 1995, 156 (3), 140-146. https://doi.org/10.1016/0022-0248(95)00296-0.

(161) Konnov, A. A.; De Ruyck, J. Kinetic Modeling of the Thermal Decomposition of Ammonia. Combust. Sci. Technol. 2000, 152 (1), 23-37. https://doi.org/10.1080/00102200008952125.

(162) Liu, S. S. Growth Kinetics and Catalytic Effects in the Vapor Phase Epitaxy of Gallium Nitride. J. Electrochem. Soc. 1978, 125 (7), 1161. https://doi.org/10.1149/1.2131641.

(163) Curtiss, L. A.; Redfern, P. C.; Raghavachari, K. Gaussian-4 Theory. J. Chem. Phys. 2007, 126 (8), 084108. https://doi.org/10.1063/1.2436888.

(164) Curtiss, L. A.; Redfern, P. C.; Raghavachari, K. Assessment of Gaussian-4 Theory for Energy Barriers. Chem. Phys. Lett. 2010, 499 (1-3), 168-172. https://doi.org/10.1016/j.cplett.2010.09.012.

(165) Purvis, G. D.; Bartlett, R. J. A Full Coupled-Cluster Singles and Doubles Model: The Inclusion of Disconnected Triples. J. Chem. Phys. 1982, 76 (4), 1910-1918. https://doi.org/10.1063/1.443164.

(166) Przhevalskii, I. N.; Karpov, S. Y.; Makarov, Y. N. Thermodynamic Properties of Group-III Nitrides and Related Species. MRS Internet Journal of Nitride Semiconductor Research. Cambridge University Press 1998. https://doi.org/10.1557/s1092578300001022.

(167) Creighton, J. R.; Wang, G. T. Reversible Adduct Formation of Trimethylgallium and Trimethylindium with Ammonia. J. Phys. Chem. A 2005, 109 (1), 133-137. https://doi.org/10.1021/jp046491h.

(168) Goerigk, L.; Grimme, S. Assessment of TD-DFT Methods and of Various Spin Scaled CIS(D) and CC2 Versions for the Treatment of Low-Lying Valence Excitations of Large Organic Dyes. J. Chem. Phys. 2010, 132 (18), 184103. https://doi.org/10.1063/1.3418614.

(169) McQuarrie, D. A.; Simon, J. D. Molecular Thermodynamics; University Science Books: Sausalito, CA, 1999.

(170) Forsberg, U.; Lundskog, A.; Kakanakova-Georgieva, A.; Ciechonski, R.; Janzén, E. Improved Hot-Wall MOCVD Growth of Highly Uniform AlGaN/GaN/HEMT Structures. J. Cryst. Growth 2009, 311 (10), 30073010. https://doi.org/10.1016/j.jcrysgro.2009.01.045.

(171) Kakanakova-Georgieva, A.; Forsberg, U.; Ivanov, I. G.; Janzén, E. Uniform 
Hot-Wall MOCVD Epitaxial Growth of 2 Inch AlGaN/GaN HEMT Structures. J. Cryst. Growth 2007, 300 (1), 100-103.

https://doi.org/10.1016/j.jcrysgro.2006.10.242.

(172) Danielsson, Ö.; Li, X.; Ojamäe, L.; Janzén, E.; Pedersen, H.; Forsberg, U. A Model for Carbon Incorporation from Trimethyl Gallium in Chemical Vapor Deposition of Gallium Nitride. J. Mater. Chem. C 2016, 4 (4), 863871. https://doi.org/10.1039/c5tc03989d.

(173) Fujita, J. I.; Ueki, R.; Miyazawa, Y.; Ichihashi, T. Graphitization at Interface between Amorphous Carbon and Liquid Gallium for Fabricating Large Area Graphene Sheets. In Journal of Vacuum Science and Technology B: Microelectronics and Nanometer Structures; American Vacuum SocietyAVS, 2009; Vol. 27, pp 3063-3066. https://doi.org/10.1116/1.3253542.

(174) Bogusławski, P.; Briggs, E. L.; Bernholc, J. Amphoteric Properties of Substitutional Carbon Impurity in GaN and AIN. Appl. Phys. Lett. 1996, 69 (2), 233-235. https://doi.org/10.1063/1.117934.

(175) Jansson, U.; Lewin, E. Sputter Deposition of Transition-Metal Carbide Films - A Critical Review from a Chemical Perspective. Thin Solid Films. Elsevier June 1, 2013, pp 1-24. https://doi.org/10.1016/j.tsf.2013.02.019.

(176) Bhuiyan, A. G.; Hashimoto, A.; Yamamoto, A. Indium Nitride (InN): A Review on Growth, Characterization and Properties. J. Appl. Phys. 2003, 94, 2779-2808.

(177) Zhang, B. L.; Sun, G. S.; Guo, Y.; Zhang, P. F.; Zhang, R. Q.; Fan, H. B.; Liu, X. L.; Yang, S. Y.; Zhu, Q. S.; Wang, Z. G. Valence Band Offset of $\mathrm{InN} / 4 \mathrm{H}-\mathrm{SiC}$ Heterojunction Measured by x-Ray Photoelectron Spectroscopy. Appl. Phys. Lett. 2008, 93, 242107.

(178) Ivanov, V. S.; Shubina, V. T.; Komissarova, T. A.; Jmerik, V. N. Metastable Nature of InN and In-Rich InGaN Alloys. J. Cryst. Growth 2014, 403, 83-89.

(179) Ruffenach, S.; Moret, M.; Briot, O.; Gil, B. Recent Advances in the MOVPE Growth of Indium Nitride. Phys. Status Solidi A 2010, 1, 9-18.

(180) Zhou, J.; Huang, Q.; Li, J.; Cai, D.; Kang, J. The InN Epitaxy via Controlling In Bilayer. Nanoscale Res. Lett. 2014, 9, 5 .

(181) George, S. M. Atomic Layer Deposition: An Overview. Chem. Rev. 2010, 110, 111-131. 
(182) Nepal, N.; Mahadik, N. A.; Nyakiti, L. O.; Qadri, S. B.; Mehl, M. J.; Hite, J. K.; Eddy, C. R. J. Epitaxial Growth of Cubic and Hexagonal InN Thin Films via Plasma-Assisted Atomic Layer Epitaxy. Cryst. Growth Des. 2013, 13, 1485-1490.

(183) Haider, A.; Kizir, S.; Biyikli, N. Low-Temperature Self-Limiting Atomic Layer Deposition of Wurtzite $\operatorname{InN}$ on $\operatorname{Si}(100)$. AIP Adv. 2016, 6, 45203.

(184) Deminskyi, P.; Rouf, P.; Ivanov, I. G.; Pedersen, H. Atomic Layer Deposition of InN Using Trimethylindium and Ammonia Plasma. J. Vac. Sci. Technol. A 2019, 37, 020926.

(185) Wakahara, A.; Tsuchiya, T.; Yoshida, A. Epitaxial Growth of Indium Nitride. J. Cryst. Growth 1990, 99, 385-389.

(186) Feng, X.; Peng, H.; Gong, J.; Wang, W.; Liu, H.; Quan, Z.; Pan, S.; Wang, L. Epitaxial Growth of InN Thin Films by Plasma-Enhanced Atomic Layer Deposition. J. Appl. Phys. 2018, 124, 243104.

(187) Bürger, H.; Cichon, J.; Goetze, U.; Wannagat, U.; Wismar, H. Beiträgezur Chemie Der Silicium-Stickstoff-Verbindungen: CVII. Darstellung, Schwingungsspektren Und Normalkoordinatenanalyse von Disilylamiden Der 3. Gruppe: $\mathrm{M}[\mathrm{N}(\mathrm{SiMe}) 2] 3 \mathrm{Mit} \mathrm{M}=\mathrm{Al}$, Ga Und In. J. Organomet. Chem. 1971, 33, 1-12.

(188) Rossetto, G.; Brianese, N.; Camporese, A.; Porchia, M.; Zanella, P.; Bertoncello, R. Synthesis and Charaeterization of Hexakis(Diethylamido)Diindium(III) and BisCyclopentadienyl(Diethylamido)Indium(III). Main Gr. Met. Chem. 1991, 14, 113-122.

(189) Petrie, M. A.; Ruhlandt-Senge, K.; Hope, H.; Power, P. P. Structural Studies of the Monomeric, Low Coordinate, Indium Amides (t$\mathrm{Bu}) 2 \mathrm{InN}(2,6-\mathrm{i}-\mathrm{Pr} 2 \mathrm{C} 6 \mathrm{H} 3) \mathrm{SiPh} 3$ and $\operatorname{In}\{\backslash$ vphantom $\{\backslash\}\} \mathrm{N}($ SiMe 3$) 2 \backslash$ vphantom $\{\backslash\{\} \backslash\} 3$. Bull. Soc. Chim. Fr. 1993, 130, 851--855.

(190) Frey, R.; Gupta, V. D.; Linti, G. Monomere Bis- Und Tris(Amide) Des Indiums. Z. anorg. allg. Chem. 1996, 622, 1060-1064.

(191) Kim, J.; Bott, S. G.; Hoffman, D. M. Synthesis of Indium Amide Compounds. Inorg. Chem. 1998, 37, 3835-3841.

(192) Gebhard, M.; Hellwig, M.; Kroll, A.; Rogalla, D.; Winter, M.; Mallick, B.; Ludwig, A.; Wiesing, M.; Wieck, A. D.; Grundmeir, G.; et al. New 
Amidinate Complexes of Indium(III): Promising CVD Precursor for Transparent and Conductive In2O3 Thin Films. Dalt. Trans. 2017, 46, $10220-10231$.

(193) Kim, S. B.; Jayaraman, A.; Chua, D.; Davis, L. M.; Zheng, S.-L.; Zhao, X.; Lee, S.; Gordon, R. G. Obtaining a Low and Wide Atomic Layer Deposition Window $\left(150-275{ }^{\circ} \mathrm{C}\right)$ for In2O3 Films Using an InIII Amidinate and H2O. Chem. Eur. J. 2018, 24, 9525-9529.

(194) Barry, S. T.; Gordon, P. G.; Ward, M. J.; Heikkila, M. J.; Monillas, W. H.; Yap, G. P. A.; Ritala, M.; Leskelä, M. Chemical Vapour Deposition of In2O3 Thin Films from a Tris-Guanidinate Indium Precursor. Dalt. Trans. 2011, 40, 9425-9430.

(195) Gebhard, M.; Hellwig, M.; Parala, H.; Xu, K.; Winter, M.; Devi, A. Indium-Tris-Guanidinates: A Promising Class of Precursors for Water Assisted Atomic Layer Deposition of In2O3 Thin Films. Dalt. Trans. 2014, 43, 937-940.

(196) McCarthy, R. F.; Weimer, M. S.; Emery, J. D.; Hock, A. S.; Martinson, A. B. F. Oxygen-Free Atomic Layer Deposition of Indium Sulfide. Appl. Mater. Interfaces 2014, 6, 12137-12145.

(197) Rouf, P.; O'Brien, N. J.; Rönnby, K.; Samii, R.; Ivanov, I. G.; Ojamäe, L.; Pedersen, H. The Endocyclic Carbon Substituent of Guanidinate and Amidinate Precursors Controlling ALD of InN Films. J. Phys. Chem. C 2019, 123, 25691-25700.

(198) Gantzel, P.; Walsh, P. J. Synthesis and Crystal Structures of Lithium and Potassium Triazenide Complexes. Inorg. Chem. 1998, 37, 3450-3451.

(199) Nimitsiriwat, N.; Gibson, V. C.; Marshall, E. L.; Takolpuckdee, P.; Tomov, A. K.; White, A. J. P.; Williams, D. J.; Elsegood, M. R.; Dale, S. H. Monoversus Bis-Chelate Formation in Triazenide and Amidinate Complexes of Magnesium and Zinc. Inorg. Chem. 2007, 46, 9988-9997.

(200) Dwyer, F. P. Chelation in Metallic Triazene Salts. J. Am. Chem. Soc. 1941, 63, 78-81.

(201) Corbett, M.; Hoskins, B. F. The Structure of Monoclinic Tris(1,3Diphenyltriazenato)Cobalt(III)- Toluene (1/1): An Octahedral Complex Containing Three Four-Membered Chelate Rings. Aust. J. Chem. 1974, 27, 665-670.

(202) Black, D. S. C.; Davis, V. C.; Deacon, G. B.; Schultze, R. J. 
Tris(Triazenido)Thallium(III) Compounds. Inorg. Chim. Acta. 1979, 37, L528.

(203) Guzei, I. A.; Liable-Sands, L. M.; Rheingold, A. L.; Winter, C. H. Synthesis and Characterization of Titanium and Zirconium Complexes Bearing Diphenyltriazenido Ligands. Polyhedron 1997, 16, 4017-4022.

(204) Ríos-Moreno, G.; Aguirre, G.; Parra-Hake, M.; Walsh, P. J. Synthesis, Characterization, and Structure of Dinuclear Copper(I) and Silver(I) Complexes of Ortho-Functionalized 1,3-Bis(Aryl)Triazenide Ligands. Polyhedron 2003, 2Q, 563.

(205) Johnson, A. L.; Willcocks, A. M.; Richards, S. P. Synthesis and Structures of Group 11 Metal Triazenide Complexes: Ligand Supported Metallophilic Interactions. Inorg. Chem. 2009, 48, 8613-8622.

(206) Lee, H. S.; Niemeyer, M. Homoleptic Heavy Alkaline Earth and Europium Triazenides. Inorg. Chem. 2010, 49, 730-735.

(207) Hinz, A.; Schulz, A.; Villinger, A.; Wolter, J.-W. Cyclo-Pnicta-Triazanes: Biradicaloids or Zwitterions? J. Am. Chem. Soc. 2015, 137, 3975-3980.

(208) Flanagan, K. R.; Parish, J. D.; Fox, M. A.; Johnson, A. L. Synthetic, Structural, and Computational Studies on Heavier Tetragen and Chalcogen Triazenide Complexes. Inorg. Chem. 2019, 58, 16660-16666.

(209) Leman, J. T.; Barron, A. R. Synthesis of 1,3-Diphenyltriazenide Complexes of Aluminium, Gallium and Indium: Crystal Structure of Tris(1,3Diphenyltriazenido)Aluminium(III). Polyhedron 1989, 8, 1909-1912.

(210) Leman, J. T.; Roman, H. A.; Barron, A. R. Five- and Six-Coordinate Organometallic Compounds of Indium. Organometallics 1993, 12, 29862990.

(211) Brinckman, F. E.; Haiss, H. S.; Robb, R. A. Metal-Nitrogen Bonding. Covalent Complexes of 1,3-Dimethyltriazene with Elements of Groups I, II, III, IV, and V. Inorg. Chem. 1965, 4, 936-942.

(212) Soussi, K.; Mishra, S.; Jeanneau, E.; Millet, J.-M.; Daniele, S. Asymmetrically Substituted Triazenes as Poor Electron Donor Ligands in the Precursor Chemistry of Iron(II) for Iron-Based Metallic and Intermetallic Nanocrystals. Dalt. Trans. 2017, 46, 13055-13064.

(213) Soussi, K.; Mishra, S.; Jeanneau, E.; Mantoux, A.; Stéphane, D. Synthesis, Characterization and Thermal Transport Properties of Heteroleptic $\mathrm{N}$-alkyltriazenide Complexes of Titanium(IV) and Niobium(V). Polyhedron 
2018, 152, 84-89.

(214) Swetha, M.; Venkata Ramana, P.; Shirodkar, S. G. Simple and Efficient Method for the Synthesis of Azides in Water-THF Solvent System. Org. Prep. Proceed. Int. 2011, 43, 348-353.

(215) Frisch, M. J.; Trucks, G. W.; Schlegel, H. B.; Scuseria, G. E.; Robb, M. A.; Cheeseman, J. R.; Scalmani, G.; Barone, V.; Petersson, G. A.; Nakatsuji, H.; et al. Gaussian 16 Revision B.01. 2016.

(216) Becke, A. D. Density-Functional Thermochemistry. III. The Role of Exact Exchange. J. Chem. Phys. 1993, 98, 5648-5652.

(217) Lee, C.; Yang, W.; Parr, R. G. Development of the Colle-Salvetti Correlation-Energy Formula into a Functional of the Electron Density. Phys. Rev. B 1988, 37, 785-789.

(218) Grimme, S.; Antony, J.; Ehrlich, S.; Krieg, H. A Consistent and Accurate Ab Initio Parametrization of Density Functional Dispersion Correction (DFT-D) for the 94 Elements H-Pu. J. Chem. Phys. 2010, 132 (15), 154104 .

(219) Weigend, F.; Ahlrichs, R. Balanced Basis Sets of Split Valence, Triple Zeta Valence and Quadruple Zeta Valence Quality for H to Rn: Design and Assessment of Accuracy. Phys. Chem. Chem. Phys. 2005, 7, 3297-3305.

(220) Metz, B.; Stoll, H.; Dolg, M. Small-Core Multiconfiguration-Dirac-HartreeFock-Adjusted Pseudopotentials for Post-d Main Group Elements: Application to $\mathrm{PbH}$ and PbO. J. Chem. Phys. 2000, 113, 2563-2569.

(221) Barry, S. T. Amidinates, Guanidinates and Iminopyrrolidinates: Understanding Precursor Thermolysis to Design a Better Ligand. Coord. Chem. Rev. 2013, 257, 3192-3201.

(222) Rubel, O.; Baranovskii, S. D. Formation Energies of Antiphase Boundaries in GaAs and GaP: An Ab Initio Study. Int. J. Mol. Sci. 2009, 10, 51045114 .

(223) Starr, D. E.; Cambell, C. T. Large Entropy Difference between Terrace and Step Sites on Surfaces. J. Am. Chem. Soc. 2008, 130, 7321-7327.

(224) Krischok, S.; Yanev, V.; Balykov, O.; Himmerlich, M.; Schaefer, J. A.; Kosiba, R.; Ecke, G.; Cimalla, I.; Cimalla, V.; Ambacher, O.; et al. Investigations of MBE Grown InN and the Influence of Sputtering on the Surface Composition. Surf. Sci. 2004, 566-568, 849-855. 
(225) Veal, T. D.; King, P. D. C.; Jefferson, P. H.; Piper, L. F. J.; McConville, C. F.; Lu, H.; Schaff, W. J.; Anderson, P. A.; Durbin, S. M.; Muto, D.; et al. In Adlayers on C-Plane InN Surfaces: A Polarity-Dependent Study by x-Ray Photoemission Spectroscopy. Phys. Rev. B 2007, 76, 75313.

(226) Wu, C. L.; Lee, H. M.; Kuo, C. T.; Chen, C. H.; Gwo, S. Absence of FermiLevel Pinning at Cleaved Nonpolar InN Surfaces. Phys. Rev. Lett. 2008, 76,75313 .

(227) Nagata, T.; Koblmüller, G.; Bierwagen, O.; Gallinat, C. S.; Speck, J. S. Surface Structure and Chemical States of A-Plane and c-Plane InN Films. Appl. Phys. Lett. 2009, 95, 132104.

(228) Wang, J. B.; Li, Z. F.; Chen, P. P.; Lu, W.; Yao, T. Raman Study of Gap Mode and Lattice Disorder Effect in InN Films Prepared by PlasmaAssisted Molecular Beam Epitaxy. Acta Mater. 2007, 55, 183-187.

(229) Camp, C.; Arnold, J. Dalton Transactions PERSPECTIVE On the NonInnocence of \&quot; Nacnacs \&quot; : Ligand-Based Reactivity in $\beta$ Diketiminate Supported Coordination Compounds. Dalt. Trans. 2016, 45 . https://doi.org/10.1039/c6dt02013e.

(230) Stalzer, M. M.; Lohr, T. L.; Marks, T. J. Synthesis, Characterization, and Thermal Properties of N-Alkyl $\beta$-Diketiminate Manganese Complexes. Inorg. Chem. 2018, 57 (6), 3017-3024. https://doi.org/10.1021/acs.inorgchem.7b02476.

(231) Bruker, A. X. S. APEX2, SAINT, and SADABS. Bruker AXS Inc.: Madison, Wisconsin 2008.

(232) Bruker, A. X. S. APEX3, SAINT, and SADABS. Bruker AXS Inc.: Madison, Wisconsin 2016.

(233) Sheldrick, G. M. SHELXT - Integrated Space-Group and Crystal-Structure Determination. Acta Crystallogr. Sect. A Found. Crystallogr. 2015, 71 (1), 3-8. https://doi.org/10.1107/S2053273314026370.

(234) Sheldrick, G. M. Crystal Structure Refinement with SHELXL. Acta Crystallogr. Sect. C Struct. Chem. 2015, 71 (1), 3-8. https://doi.org/10.1107/S2053229614024218.

(235) Hübschle, C. B.; Sheldrick, G. M.; Dittrich, B. ShelXle: A Qt Graphical User Interface for SHELXL. J. Appl. Crystallogr. 2011, 44 (6), 1281-1284. https://doi.org/10.1107/S0021889811043202.

(236) Dolomanov, O. V.; Bourhis, L. J.; Gildea, R. J.; Howard, J. A. K.; 
Puschmann, H. OLEX2: A Complete Structure Solution, Refinement and Analysis Program. J. Appl. Crystallogr. 2009, 42 (2), 339-341. https://doi.org/10.1107/S0021889808042726.

(237) Farrugia, L. J. WinGX and ORTEP for Windows: An Update. J. Appl. Crystallogr. 2012, 45 (4), 849-854. https://doi.org/10.1107/S0021889812029111.

(238) Spek, A. L. CheckCIF Validation ALERTS: What They Mean and How to Respond. Acta Crystallogr. Sect. E Crystallogr. Commun. 2020, 76 (1), 111. https://doi.org/10.1107/S2056989019016244.

(239) Dey, G.; Elliott, S. D. Copper(I) Carbene Hydride Complexes Acting Both as Reducing Agent and Precursor for Cu ALD: A Study through Density Functional Theory. Theor. Chem. Acc. 2014, 133 (1), 1-7. https://doi.org/10.1007/s00214-013-1416-y.

(240) Ashby, E. C.; Kovar, R. A. Animoalanes. Direct Synthesis from Aluminum, Hydrogen, and Secondary Amines. Structure and Stability Elucidation by Nuclear Magnetic Resonance, Infrared Spectra, and Differential Thermal and Thermal Gravimetric Analysis. Inorg. Chem. 1971, 10 (5), 893-899. https://doi.org/10.1021/ic50099a006.

(241) Jana, B.; Uhl, W. New Aluminum and Gallium Complexes of $\beta$ Diketiminato and $\beta$-Ketiminato Ligands. Inorganica Chim. Acta 2017, 455, 61-69. https://doi.org/10.1016/J.ICA.2016.10.013.

(242) Ruff, J. K.; Hawthorne, M. F. The Amine Complexes of Aluminum Hydride. I. J. Am. Chem. Soc. 1960, 82 (9), 2141-2144. https://doi.org/10.1021/ja01494a013.

(243) Nilsson, D.; Janzen, E.; Kakanakova-Georgieva, A. Lattice Parameters of AlN Bulk, Homoepitaxial and Heteroepitaxial Material - IOPscience. $J$. Phys. D. Appl. Phys. 2016, 49 (17).

(244) Rouf, P.; O’Brien, N. J.; Buttera, S. C.; Martinovic, I.; Bakhit, B.; Martinsson, E.; Palisaitis, J.; Hsu, C.-W.; Pedersen, H. Epitaxial GaN Using $\mathrm{Ga}(\mathrm{NMe} 2) 3$ and NH 3 Plasma by Atomic Layer Deposition. $J$. Mater. Chem. C 2020, 3, 1101-1134. https://doi.org/10.1039/D0TC02085K. 